Aus der Abteilung Diagnostische und Interventionelle Neuroradiologie

(Prof. Dr. med. M. Knauth)

im Zentrum Radiologie

der Medizinischen Fakultät der Universität Göttingen

\title{
Mechanische Rekanalisation bei akutem ischämischen Schlaganfall durch Aspirationsthrombektomie mit dem Penumbra System
}

Eine retrospektive Studie

\author{
INAUGURAL-DISSERTATION \\ zur Erlangung des Doktorgrades \\ der Medizinischen Fakultät \\ der Georg-August-Universität zu Göttingen
}

vorgelegt von

Andreas Kreusch

aus Tübingen 
Dekan: Prof. Dr. rer. nat. H. Kroemer

I. Berichterstatter: Prof. Dr. med. M. Knauth

II. Berichterstatter: Prof. Dr. med. G. Trendelenburg

Tag der mündlichen Prüfung: 4. Juni 2013 


\section{Inhaltsverzeichnis}

1. Einleitung

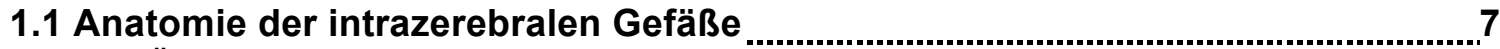

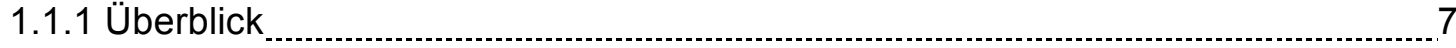

1.1.2 Vorderer Gefäßkreislauf

1.1.3 Hinterer Gefäßkreislauf 10

1.1.4. Circulus arteriosus Willisii

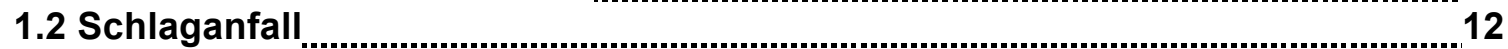

1.2.1 Definition und Epidemiologie

1.2.2 Ätiologie und Pathogenese

1.2.3 Risikofaktoren

1.2.4 Klinik

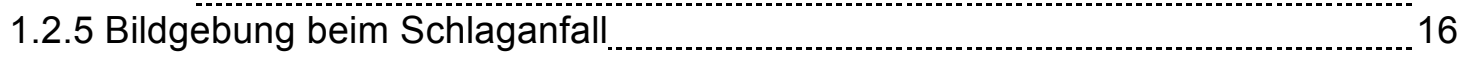

1.2.5.1 Pathophysiologie: Das Penumbra-Konzept 16

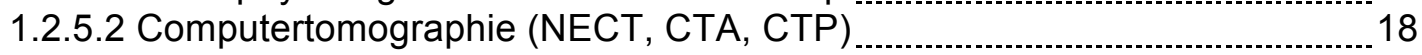

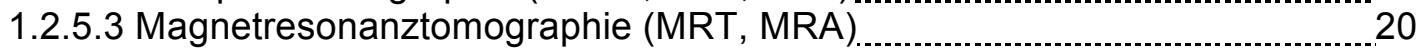

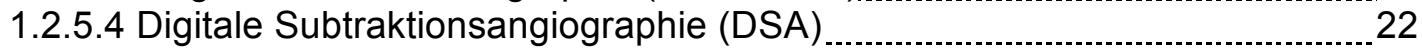

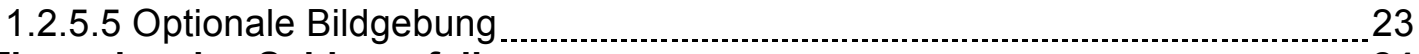

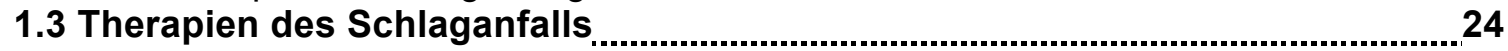

1.3.1 Notfallmedizinische Behandlung.

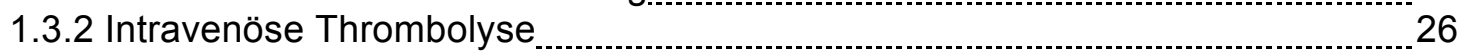

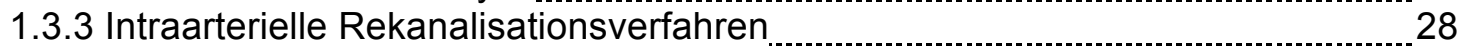

1.3.3.1 Intraarterielle Thrombolyse

1.3.3.2 Mechanische Rekanalisation

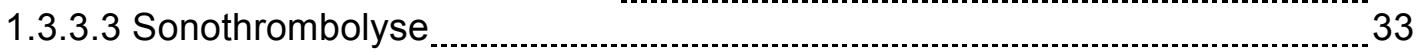

1.3.3.4 Kombinationstherapien

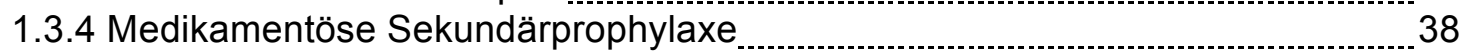

1.3.5 Vorbeugung und Behandlung von Komplikationen

1.3.6 Rehabilitation

1.4 Aktueller Stand der Forschung.

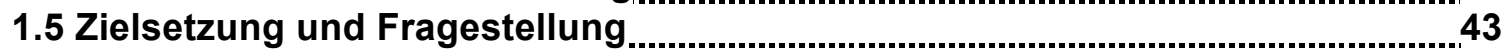

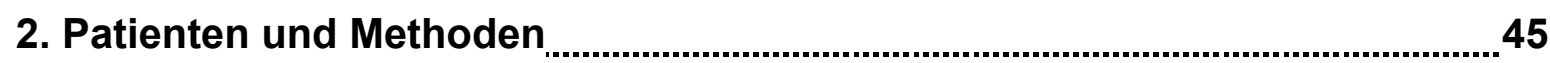

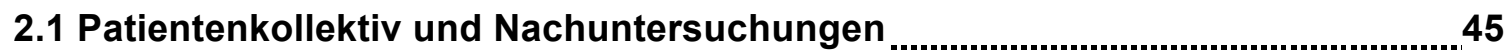

2.2 Diagnostik

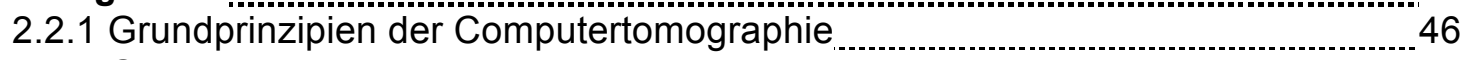

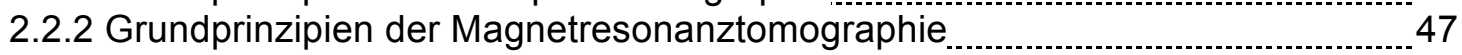

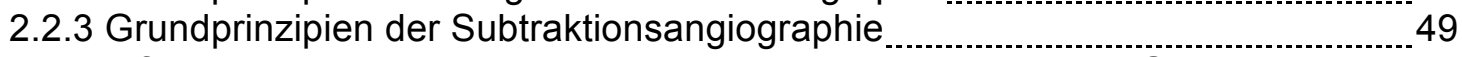

2.3 Ablauf der mechanischen Rekanalisation mit dem Penumbra System ...............51

2.3.1 Indikationen

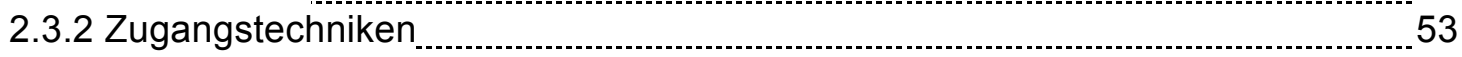

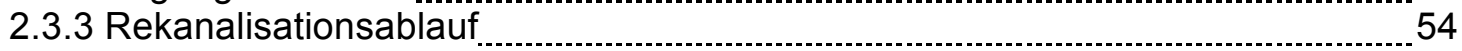

2.4 Weiterbehandlung.

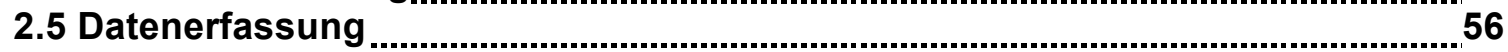

2.5.1 NIH Stroke Scale (NIHSS)

2.5.2 Modified Rankin Scale (mRS)

2.5.3 Modified Thrombolysis In Myocardial Infarction (mTIMI) Score _.............................. 58 
2.5.4 Thrombolysis in Cerebral Infarction ( $\mathrm{TICl}$ ) Score

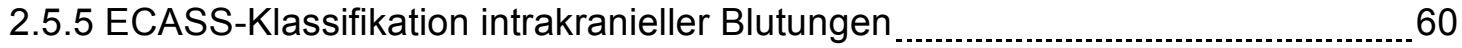

2.6 Datenauswertung

2.7 Ethik

3. Ergebnisse

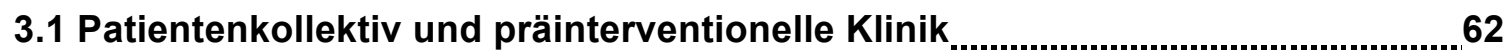

3.1.1 Alters- und Geschlechtsverteilung

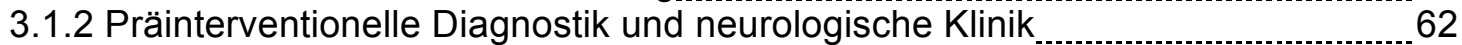

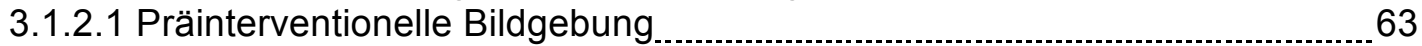

3.1.2.2 Zeitverlauf 63

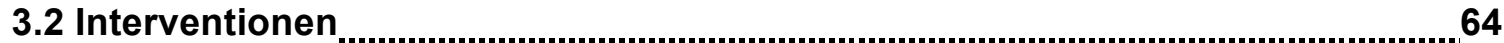

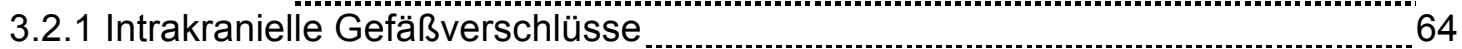

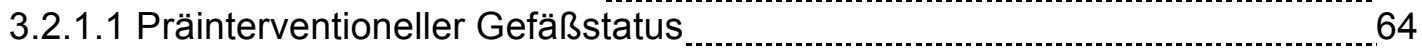

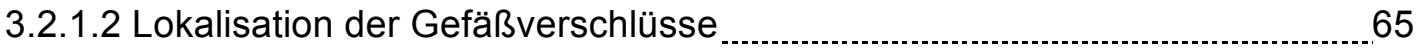

3.2.2 Materialeinsatz

3.2.3 Kombinationstherapien \& rt-PA-Einsatz 66

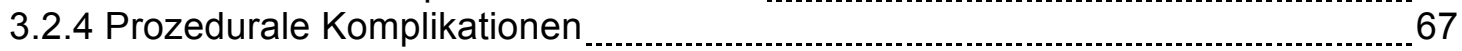

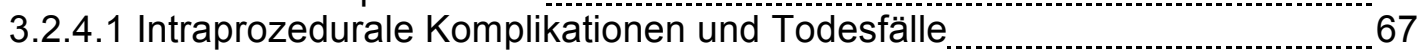

3.2.4.2 Postprozedurale Komplikationen und Todesfälle $\ldots \ldots \ldots$

3.2.5 Vaskuläre Ergebnisse

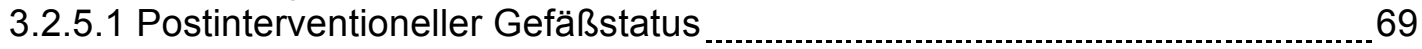

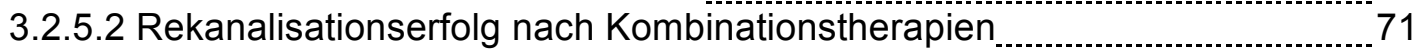

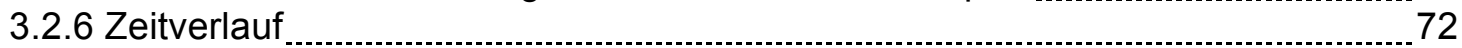

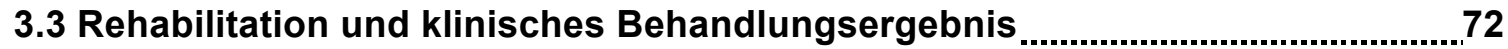

3.3.1 Rehabilitationsverläufe $\ldots$

3.3.2 Klinische Ergebnisse

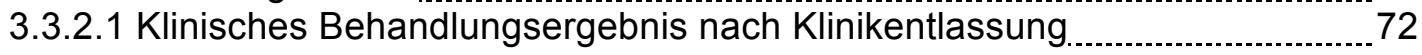

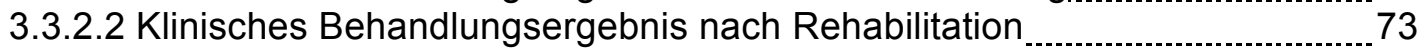

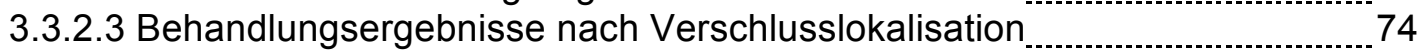

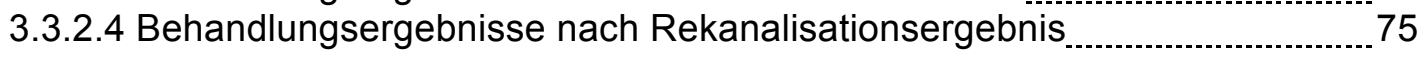

3.3.2.5 Behandlungsergebnisse nach früher bzw. später Rekanalisation .................... 75

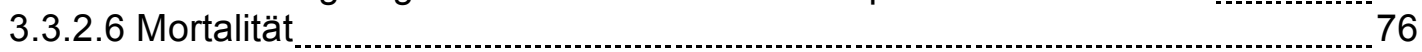

3.4 Fallbeispiele

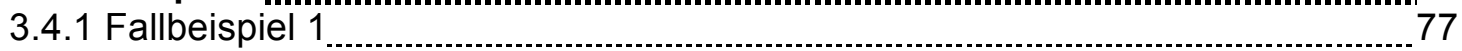

3.4.2 Fallbeispiel 2

4. Diskussion

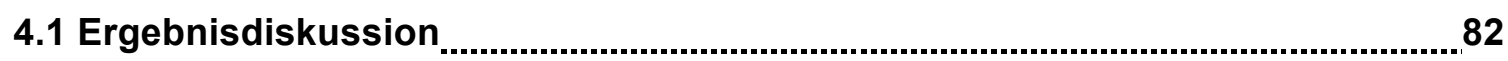

4.1.1 Rekanalisationsergebnisse 82

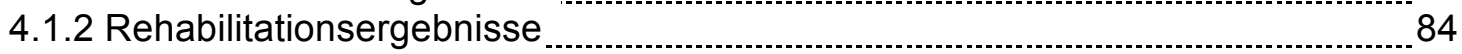

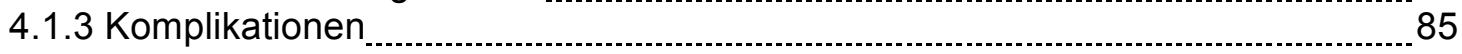

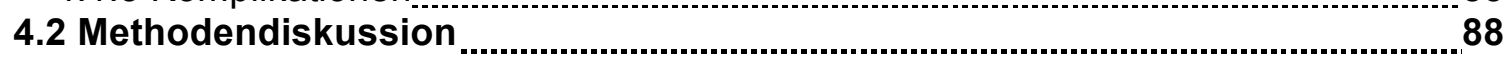

4.2.1 Studiendesign

4.2.2 Patientenauswahl

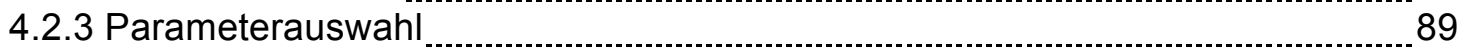

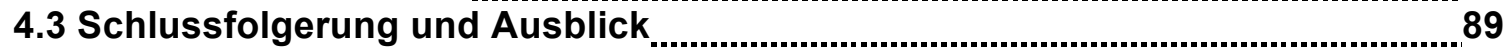

5. Zusammenfassung 
6. Anhang

Anhang 1: National Institute of Health Stroke Scale (NIHSS)

Anhang 2: Modified Rankin Scale (mRS) $\ldots 102$

Anhang 3: Modified Thrombolysis In Myocardial Infarction (mTIMI) Bewertungsskala102

Anhang 4: Thrombolysis in Cerebral Infarction (TICl) Bewertungsskala .................... 102

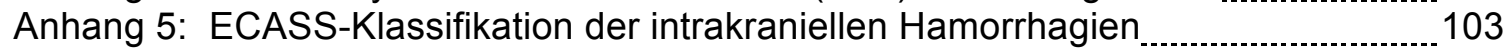

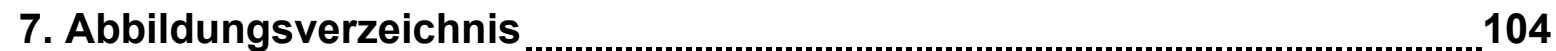

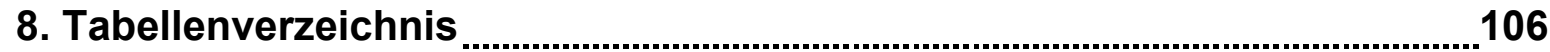

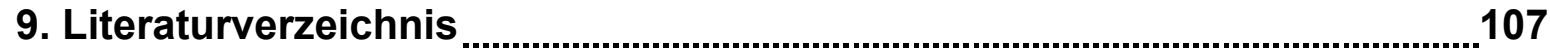




\section{Abkürzungsverzeichnis}

ACA

ACT

$A D C$

BA

CBF

CBV

CCA

CCT

CTA

CTP

DSA

DWI

ECASS

FDA

FPD

HE

$\mathrm{HI}$

IAT

ICA

$\mathrm{ICH}$

IQR

IVT

$\mathrm{KM}$

MCA

MRA

mRS

MRT

MTT

mTIMI

NECT

NIHSS

PCA
Arteria cerebri anterior

Angiographische CT

Apparent Diffusion Coefficient („scheinbarer Diffusionskoeffizient“)

Arteria basilaris

zerebraler Blutfluss

zerebrales Blutvolumen

Arteria carotis communis

kranielle Computertomographie

CT-Angiographie

Perfusions-CT

Digitale Subtraktionsangiographie

Diffusion Weighted Imaging (Diffusions-gewichtete MRT-Bildgebung)

European Cooperative Acute Stroke Study

Food and Drug Organisation

Flachbild-Detektor (flat-panel detector)

Hounsfield-Einheiten

hämorrhagischer Infarkt

intraarterielle Thrombolyse

Arteria carotis interna

intrakranielle Blutung (engl. intracranial hemorrhage)

Interquartilsabstand (engl. interquartile range)

intravenöse Thrombolyse

Kontrastmittel

Arteria cerebri media

Magnetresonanzangiographie

modified Rankin Scale

Magnetresonanztomographie

mediane Kontastmittel-Transitzeit (engl. mean transit time)

modified Thrombolysis in Myocardial Infarction

native Computertomographie

National Institutes of Health Stroke Scale

Arteria cerebri posterior 
$\mathrm{PH} \quad$ parenchymales Hämatom

PRC Penumbra Reperfusion Catheter

PS Penumbra System

PWI Perfusion Weighted Imaging (Perfusions-gewichtete MRT-Bildgebung)

rt-PA recombinant tissue plasminogen activator

SD Standardabweichung (engl. standard deviation)

$\mathrm{SICH}$ symptomatische intrakranielle Blutung (engl. symptomatic intracranial hemorrhage)

TIA transitorisch ischämische Attacke

$\mathrm{TICl} \quad$ Thrombolysis in Cerebral Infarction

TIMI Thrombolysis in Myocardial Infarction

TTP Zeit bis zum Kontrastmittelmaximum (engl. time to peak)

VA Arteria vertebralis 


\section{Einleitung}

Der Schlaganfall ist in Deutschland die häufigste neurologische Erkrankung, dritthäufigste Todesursache und häufigste Ursache für lebenslange Behinderung im Erwachsenenalter (Kolominsky-Rabas und Heuschmann 2002). Weltweit rangiert der Schlaganfall als die zweithäufigste Todesursache $(9,7 \%$ aller Todesfälle) hinter dem ischämischen Herzinfarkt (12,2 \%) (WHO 2008).

Den Goldstandard in der Therapie des akuten ischämischen Schlaganfalls stellt heute die medikamentöse intravenöse Thrombolyse mit Alteplase (rt-PA) dar. Sie ist weit verbreitet und steht nach dem Ausschluss einer Blutung schnell zur Verfügung. Wenn diese Therapie innerhalb von 4,5 Stunden nach Symptombeginn durchgeführt wird, führt sie zu einem verbesserten klinischen Endergebnis nach 90 Tagen (Hacke et al. 2008). Neben dem begrenzten Zeitfenster liegt die Hauptlimitation in der reduzierten Effizienz bei langstreckigen Gefäßthromben, die medikamentös oft nicht vollständig aufgelöst werden können. Die Rekanalisationswahrscheinlichkeit durch Alteplase beträgt $<1 \%$, wenn die Thrombuslänge $8 \mathrm{~mm}$ überschreitet (Riedel et al. 2011).

Eine erfolgreiche Rekanalisation ist ein wichtiger Grundstein für die Genesung und korreliert mit gutem funktionellem Outcome und geringerer Mortalität (Rha und Saver 2007). Aber auch nicht alle Patienten profitieren gleichermaßen von der Gefäßwiedereröffnung. Intraarterielle, besonders mechanische Verfahren führen schnell zu deutlich höheren Rekanalisationsraten (80-100 \%). Für Patienten mit kontraindizierter oder fehlgeschlagener intravenöser Thrombolyse (IVT) stellt die mechanische Rekanalisation eine potentiell wichtige Option dar, die allein oder in Kombination mit der systemischen Lyse zur vollständigen Rekanalisation des Gefäßes eingesetzt werden kann. Das Penumbra System ist ein neues mechanisches Instrument für die kontinuierliche Aspirationsthrombektomie bei akutem ischämischen Schlaganfall aufgrund von langstreckigen Hirngefäßverschlüssen.

In dieser Arbeit werden die Erfahrungen der Abteilung für Neuroradiologie der Universitätsmedizin Göttingen mit der mechanischen Rekanalisation von Schlaganfallpatienten mit dem neuartigen Penumbra System geschildert. Es erfolgte eine retrospektive Datenerfassung der neuroradiologischen und klinischen Daten von 91 Patienten von der Klinikaufnahme bis zum Abschluss der Rehabilitation. 


\subsection{Anatomie der intrazerebralen Gefäße}

\subsection{1 Überblick}

Die arterielle Blutversorgung des Gehirns lässt sich in einen vorderen und einen hinteren Gefäßkreislauf einteilen.

Der vordere Kreislauf wird beidseitig von den Aa. carotides internae (ICA) gespeist. Sie entspringen jeweils aus den Aa. carotides communes (CCA), wobei die linke CCA direkt aus dem Aortenbogen abgeht und die rechte CCA aus dem Truncus brachiocephalicus hervorgeht.

Der hintere Kreislauf entspringt den Aa. vertebrales (VA), die beidseits aus den Aa. subclaviae entstammen und sich im Schädelinneren zur A. basilaris (BA) vereinigen. Als intrakraniell werden alle Gefäße definiert, nachdem sie in die Schädelhöhle eingetreten sind. Der Eintritt erfolgt bei den ICA durch den Canalis caroticus und bei den VA durch das Formen magnum, beide an der Schädelbasis gelegen.

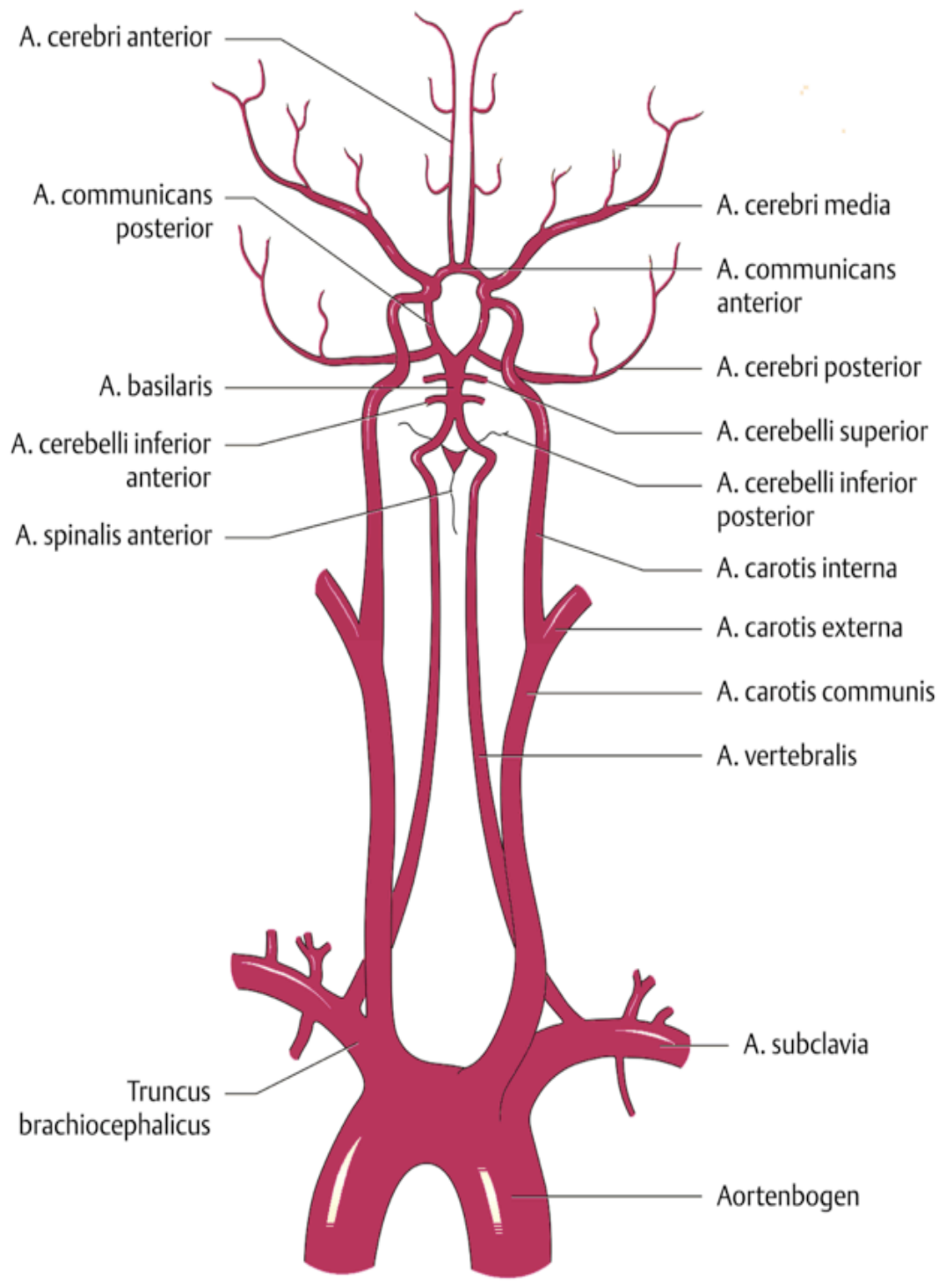

Abbildung 1 - Übersicht der supraaortalen Gefäße. Modifiziert nach Siegenthaler und Blum 2006, Seite 1081. 


\subsubsection{Vorderer Gefäßkreislauf}

Die CCA teilt sich etwa in Höhe des 4./5. Halswirbels in die Gehirn-versorgende A. carotis interna und die A. carotis externa, die vorwiegend die Kopfweichteile und oberen Halsorgane (Pharynx, Larynx, Glandula thyroidea) versorgt.

In ihrem extrakraniellen Verlauf von der Carotis-Bifurkation bis zu ihrem Eintritt in das Os petrosum gibt die ICA keine Gefäßäste ab, dieser Abschnitt wird auch als Pars cervicals (C1) bezeichnet. Der weitere intrakranielle Verlauf wird bis zur ICAAufteilung in A. cerebri media (MCA) und A. cerebri anterior (ACA) in weitere 6 Abschnitte unterteilt. Das C2-Segment (auch: Pars petrosa) bezeichnet den Verlauf im Canalis carotis im Os temporale und der anschließende kurze C3-Abschnitt (auch: Pars lacera) befindet sich unterhalb des Foramen lacerum. Die Pars cavernosa (C4) verläuft durch den Sinus caroticus, bevor das C5-Segment (clinoides Segment) komplett intradural liegt. Der folgende C6-Abschnitt (auch: Pars ophthalmica) stellt den ersten subarachnoidalen Abschnitt dar und ihm entspringt die A. ophthalmica. Die Pars communicans (C7) reicht vom Abgang der A. communicans posterior bis zur aufzweigenden Mündung der ICA in die MCA und ACA. Die nahezu rechtwinklige Gabelung der MCA und ACA wird auch Carotis-T genannt.

Die ACA verläuft als medialer, kleinerer Ast der ICA-Bifurkation über den Nervus opticus und dann in der Fissura longitudinalis um das Corpus callosum. Sie kann in ein horizontales (A1), ein vertikales (A2) und ein distales Segment (A3) eingeteilt werden und ist über den Ramus communicans anterior mit der kontralateralen ACA verbunden. Sie versorgt hauptsächlich die mediale Hemisphäre des Cerebrums im Bereich der Mantelkante von frontal bis zum Sulcus parieto-occipitalis, sowie über perforierende Äste einen medialen Anteil der Basalganglien, das Corpus callosum sowie den anterioren Schenkel der Capsula interna.

Die MCA ist der größte Endast der ICA, verläuft lateralseitig der ACA und wird in die Segmente M1-4 unterteilt. Der M1-Abschnitt erstreckt sich von der ICA-Bifurkation bis zum Sulcus lateralis (Sylvische Fissur), in dem die Arterie dann aufwärts zieht (MCA-Knie). Über die Insel verläuft das M2-Segment, bevor die MCA im M3Abschnitt das Operculum passiert, wieder aus dem Sulcus lateralis oben austritt und die distalen M4-Abschnitte über die laterale Fläche der Cortex cerebri ziehen.

Die MCA versorgt den größten Teil der lateralen Großhirnhemisphären sowie über abgehende Gefäßbäume die medialen Basalganglien sowie die Capsula interna und externa. 


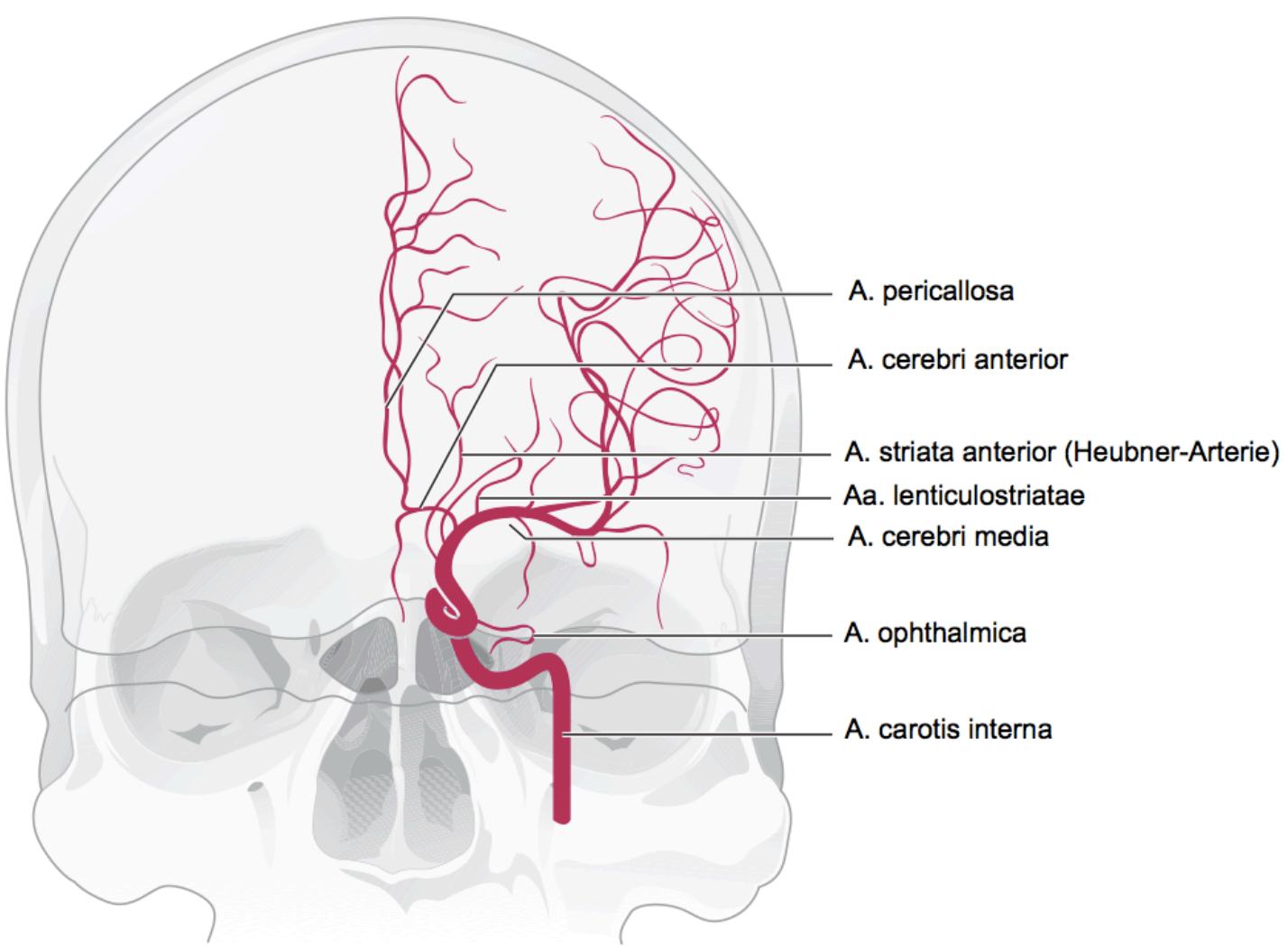

Abbildung 2 - Übersicht der intrakraniellen vorderen Zirkulation in anteroposteriorer Sicht. Modifiziert nach Caplan 2009, Seite 31.

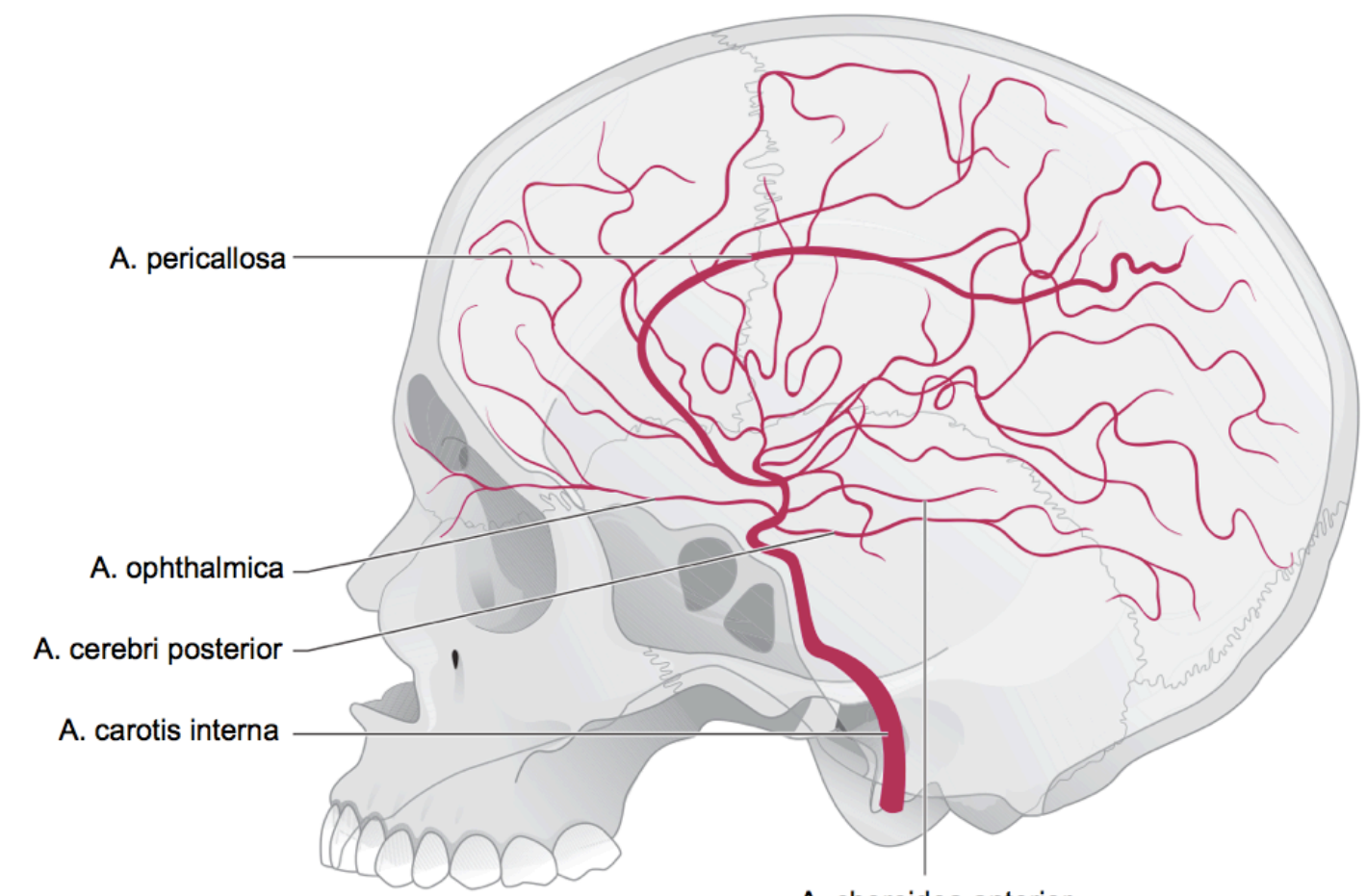

A. choroidea anterior

Abbildung 3 - Übersicht der intrakraniellen vorderen Zirkulation in lateraler Sicht. Modifiziert nach Caplan 2009, Seite 31. 


\subsubsection{Hinterer Gefäßkreislauf}

Der hintere Kreislauf wird aus den paarigen Aa. vertebrales (VA) gespeist, welche beidseitig aus den Aa. subclaviae entspringen und im extrakraniellem Verlauf durch die Foramina transversalia der Corpora vertebrae ziehen, bevor sie beidseits durch das Foramen magnum in die Schädelhöhle eintreten. Der extrakranielle Verlauf kann in 3 Abschnitte unterteilt werden: Segment V1 entspricht dem Ursprung aus der A. subclavia bis Corpus vertebrae C6, Segment V2 reicht von Corpus vertebrae C6 bis zum Axis und die Strecke vom Atlas bis zum Foramen magnum wird als V3 bezeichnet. Auf Höhe des Pons-Unterrandes vereinigen sich die intrakraniellen Abschnitte V4 der beiden VA zur unpaaren A. basilaris (BA).

Am Pons-Oberrand teilt sich die $B A$ in ihre paarigen Endäste, die $A a$. cerebri posteriores (PCA) als Teil des Circulus arteriosus Willisii. Die jeweilige PCA verläuft im P1-Segment in der Cisterna interpeduncularis bis zum Zusammenfluss mit dem ipsilateralen Ramus communicans posterior. Das P2-Segment zieht um den Pedunculus cerebri, während sich der folgende P3-Abschnitt von der Lamina quadrigemina bis zum Sulcus calcarinus erstreckt. Distale P4-Äste liegen oberhalb des Tentoriums im Sulcus calcarinus.

Die PCA versorgt den Lobus occipitalis, einen Teil des Lobus parietalis sowie Anteile des Hirnstammes und des Thalamus.

Die paarig aus der BA entspringenden Aa. cerebelli inferiores anteriores (AICA) und Aa. cerebelli superiores (SUCA) perfundieren das mittlere und obere Cerebellum. Die aus dem V4-Segment entspringenden Aa. cerebelli inferiores posteriores (PICA) versorgen ebenfalls den unteren Teil des Cerebellums.

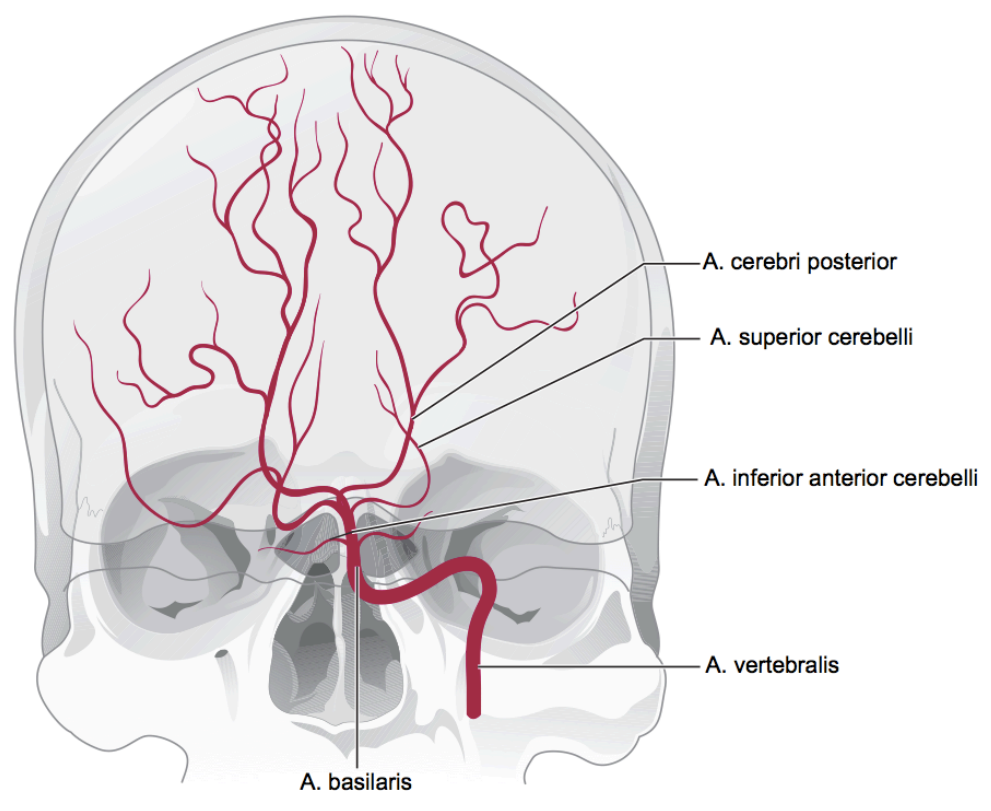

Abbildung 4 - Übersicht der intrakraniellen hinteren Zirkulation in anteroposteriorer Sicht. Modifiziert nach Caplan 2009, Seite 37 . 


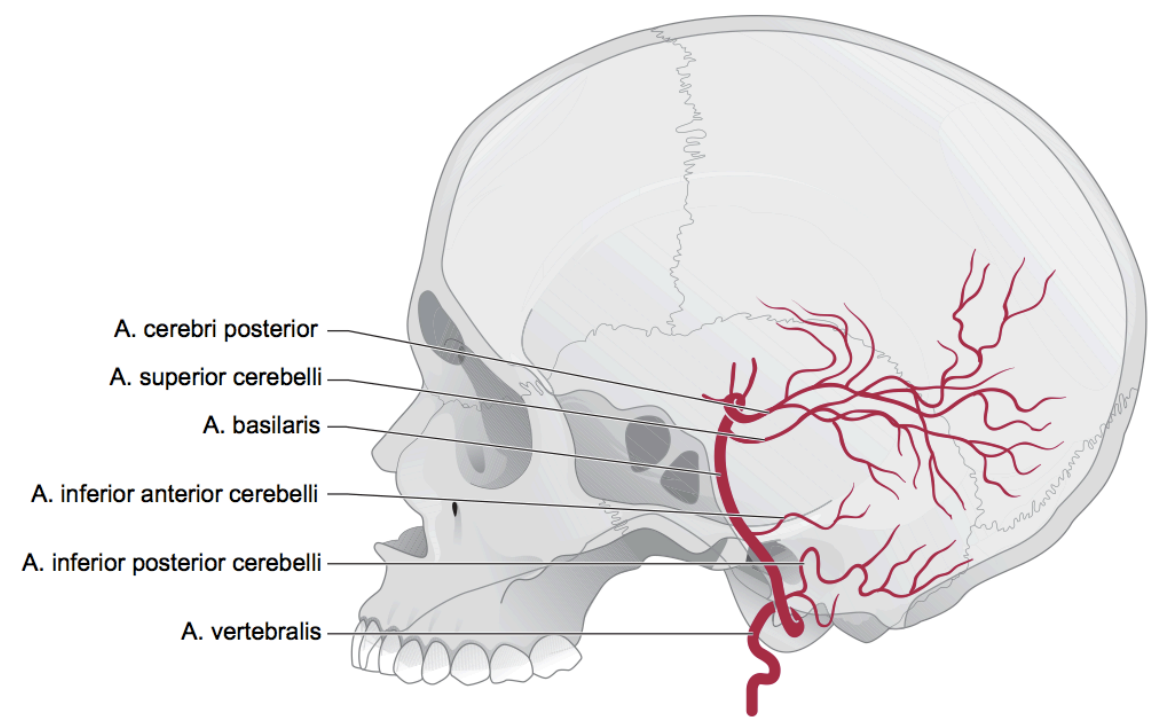

Abbildung 5 -

Übersicht der intrakraniellen hinteren

Zirkulation in lateraler Sicht. Modifiziert nach Caplan 2009, Seite 37.

\subsubsection{Circulus arteriosus Willisii}

Eine Kommunikation zwischen dem vorderen und dem hinteren Kreislauf wird durch die Rami communicantes hergestellt, der entstehende vollständige Ring an der Hirnbasis um den Hypophysenstiel und das Chiasma opticum herum wird Circulus arteriosus Willisii genannt.

Der Ramus communicans anterior stellt eine Verbindung zwischen rechter und linker ACA her, die paarigen Rami communicantes posteriores verbinden die jeweilige ICA mit der ipsilateralen PCA.

Der Circulus arteriosus Willisii stellt neben den physiologischen Anastomosen der intra- und extrakraniellen Gefäße und leptomeningealen Anastomosen den wichtigsten kollateralen Versorgungsweg dar, der den Verschluss eines oder manchmal auch mehrerer zuführender extrakranieller Gefäße ausgleichen kann.

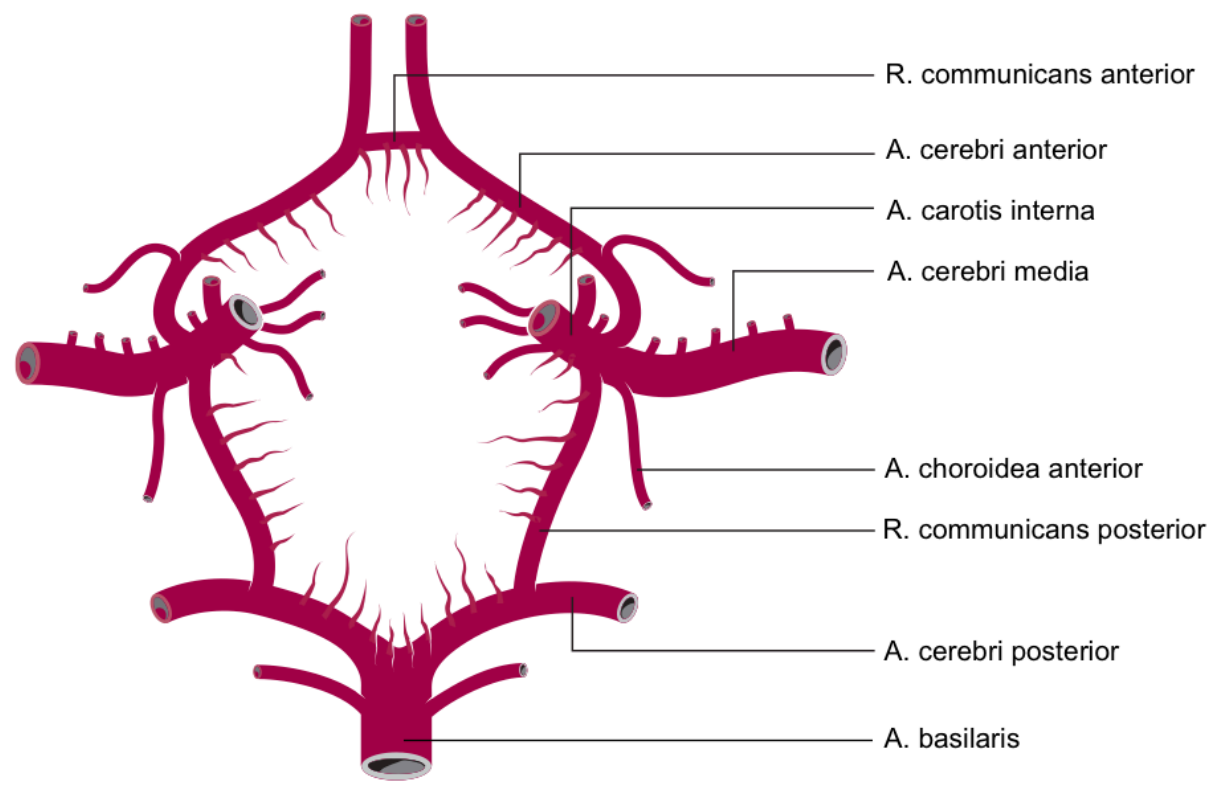

Abbildung 6 Übersicht über den Circulus arteriosus Willisii. Modifiziert nach Caplan 2009, Seite 39. 


\subsection{Schlaganfall}

\subsubsection{Definition und Epidemiologie}

Ein Schlaganfall (lat. Apoplexia cerebri) ist definiert als eine akut auftretende Funktionsstörung des Gehirns. Diese Funktionseinschränkung kann entweder durch eine kritische Blutminderversorgung des Gehirns (Ischämie) oder durch eine intrakranielle Blutung bedingt sein.

Der Schlaganfall ist in Deutschland die häufigste neurologische Erkrankung, dritthäufigste Todesursache und häufigste Einzelursache für lebenslange Behinderung. Weltweit rangiert der Schlaganfall als die zweithäufigste Todesursache (9,7 \% aller Todesfälle) hinter dem ischämischen Herzinfarkt (12,2 \%) (WHO 2008).

In Deutschland wird die Inzidenz der Schlaganfälle mit jährlich etwa 182/100.000 Einwohner angegeben. Die Letalität innerhalb des ersten Jahres nach Schlaganfall beträgt $37,3 \%$. Deutsche Männer sind häufiger als Frauen betroffen, wobei geschlechtsunabhängig $51 \%$ der Schlaganfälle sich in der Personengruppe $>75$ Jahre ereignen (Kolominsky-Rabas und Heuschmann 2002). Etwa 700.000 Menschen leben hierzulande mit den Folgen eines Schlaganfalls.

Bedingt durch den steigenden Altersdurchschnitt der deutschen Bevölkerung wird mit einer kontinuierlichen Zunahme der Fälle bis zum Jahr 2025 gerechnet, womit eine steigende Kostenbelastung für das Gesundheitssystem zu erwarten ist. Im Jahr 2006 wurden die entstandenen lebenslangen Gesamtausgaben pro Schlaganfall mit 43.129€ pro Patient in Deutschland angegeben (Kolominsky-Rabas et al. 2006).

Erkenntnisse zur Pathophysiologie bzw. zu therapeutischen Interventionsmöglichkeiten haben somit nicht nur akademische, sondern auch hohe sozioökonomische Relevanz.

\subsection{2 Ätiologie und Pathogenese}

Der unscharf definierte Begriff „Schlaganfall“ fasst akute neurologische Defizite verschiedener vaskulärer Ursachen zusammen: Etwa 80 \% der Schlaganfälle sind ischämische Infarkte, die zu einer Ischämie eines umschriebenen Gefäßterritoriums des Gehirns führen. $10 \%$ der Fälle liegt eine intrazerebrale Blutung zu Grunde, weitere $3 \%$ sind durch subarachnoidale Blutungen bedingt (Lloyd-Jones et al. 2010). Bei primär ischämischen Infarkten kann es ebenfalls zu sekundären Blutungen im Infarktgebiet (hämorrhagische Infarzierung) kommen. Eine sichere Unterscheidung zwischen Ischämie und Blutung ist anhand klinischer Kriterien allein nicht sicher 
möglich, sodass eine Bildgebung zur differentialtherapeutischen Entscheidung zwingend erforderlich ist.

In sehr wenigen Fällen ist eine nicht-vaskuläre Ursache ausschlaggebend.

Die vorherrschenden ischämischen Schlaganfälle lassen sich anhand der Trial of ORG 10172 in Acute Stroke Treatment (TOAST) Classification (Adams et al. 1993) in 5 ätiologische Kategorien einteilen:

1. Kardioembolien (26\%)

2. Mikroangiopathien (24\%)

3. atherothrombotische Makroangiopathien (12\%)

4. Infarkte unklarer Ursache (37 \%)

5. Infarkte anderer Ursache (2\%).

Die prozentualen Verteilungen entstammen dem bevölkerungsbezogenen Schlaganfallsregister "Erlanger Schlaganfall Projekt (ESPro)“ des Zeitraumes 19942008 (Dietl et al. 2009).

Kardioembolien liegen oftmals Vorhofflimmern und Herzinsuffizienz als Pathomechanismen zugrunde. Bei Mikroangiopathien kann anamnestisch meist ein Hypertonus oder ein Diabetes mellitus als mögliche Ursache diagnostiziert werden. Die Kategorie der atherothrombotischen Makroangiopathie ist durch höhergradige Stenosen oder durch den Verschluss hirnzuführender Gefäße gekennzeichnet. Eine sichere Klassifizierung ist bei Hirninfarkten ungeklärter Ätiologie meist durch mehrere konkurrierende Ursachen (z.B. Stenose der Arteria carotis interna bei gleichzeitiger kardialer Emboliequelle) nicht möglich. In Ausnahmefällen kann ein Hirninfarkt aufgrund anderer Ätiologie entstehen, beispielsweise durch bakterielle Entzündungen oder hämatologische Erkrankungen.

Für die Entstehung von intravasalen Thromben ist die Atherosklerose, in den Arterien Arteriosklerose genannt, von großer Bedeutung. Im Rahmen der degenerativen Intimaveränderungen der Gefäßwand besteht die Gefahr von Thrombusbildungen. Die Endotheldysfunktion sowie eventuelle Skleroseplaques begünstigen eine Thrombozytenagglutination und Flussreduzierung im betreffenden Gefäßabschnitt.

Die Folge können ein thrombotischer Gefäßverschluss oder eine Loslösung des Thrombus als Embolus von arteriosklerotischen Plaques sein. Der Ursprung einer zerebralen Embolie kann arterio-arteriell (vorgeschaltete Stenose), kardial (Vorhofflimmern, Herzklappenkrankheiten, akuter Myokardinfarkt) oder bei einem kardialen Septumdefekt auch venös (paradoxe Embolie) sein. 
Beim Schlaganfall unterscheidet man ätiopathogenetisch eine Makroangiopathie mit Territorial-, Grenzzonen- und Endstrominfarkten von einer Mikroangiopathie mit lakunären Infarkten und subkortikaler arteriosklerotischer Enzephalopathie (SAE).

\subsubsection{Risikofaktoren}

Der Schlaganfall ist eine polyätiologische Erkrankung, dessen Risikofaktoren für ein Auftreten eines ischämischen oder hämorrhagischen Schlaganfalls aber weitgehend mit den Risikofaktoren für kardiovaskuläre Erkrankungen übereinstimmen. Die Bedeutung der einzelnen Risikofaktoren ist jedoch sehr abhängig vom SchlaganfallSubtyp (Grau et al. 2001).

Durch Studien konnte der signifikante Einfluss einer Vielzahl von Risikofaktoren für die Genese eines Schlaganfalls gut belegt werden (Grau et al. 2001; Petty et al. 1999; Sacco 1997).

Man kann die Risikofaktoren in nicht-modifizierbare und modifizierbare Risikofaktoren einteilen:

Zu den nichtmodifizierbaren Risikofaktoren zählen ein hohes Alter, das männliche Geschlecht sowie die genetische Disposition zu kardio- und zerebrovaskulären Krankheiten.

Der wichtigste und auch am besten zu beeinflussende Risikofaktor ist die arterielle Hypertonie, die das relative Risiko für das Erleiden eines Schlaganfalls um das 3- bis 5fache steigert. Ein Anstieg des systolischen Blutdrucks um $10 \mathrm{mmHg}$ erhöht das Risiko, einen Schlaganfall zu erleiden, um $10 \%$. Bei der Prävalenz des Bluthochdrucks wird die enorme Bedeutung für die Schlaganfallsentstehung deutlich. Weitere modifizierbare Risikofaktoren sind: Diabetes mellitus Typ I und II, Übergewicht, Bewegungsmangel, Vorhofflimmern, persistierendes offenes Foramen ovale, koronare Herzkrankheit, vorherige transiente ischämische Attacke, Hyperlipidämie, Hypercholesterinämie, Hypercysteinämie und Gerinnungsstörungen. Rauchen, Alkoholabusus und die Einnahme oraler Kontrazeptiva erhöhen ebenfalls das Schlaganfallsrisiko.

\subsubsection{Klinik}

Die Ausprägung der klinisch-neurologischen Symptome eines Schlaganfalls ist abhängig von der Lokalisation des Gefäßverschlusses und der Zeitdauer der Minderperfusion distal davon gelegener Hirnareale. Typischerweise kommt es zu 
einem plötzlichen Auftreten mehrerer Symptome, die im Verlauf fluktuieren können. Ein Rückschluss von der Symptomatik auf die differenzierte Ursache eines Schlaganfalls ist jedoch keinesfalls möglich.

Wichtige Leitsymptome des Schlaganfalls sind: Hemiparese und/oder Hemihypästhesie einer Körperhälfte, Aphasie und/oder Dysarthrie, Gesichtsfelddefekte (meist Hemianopsie), Neglect, Herdblick, Bewusstseinstrübung (Somnolenz bis Koma), Übelkeit, Erbrechen, Hirnnervenausfälle und pathologische Babinski-Reflexe.

Entsprechend dem betroffenen Gefäßversorgungsareal kommt es zu richtungsweisenden neurologischen Ausfällen:

Bei einer Ischämie im Versorgungsbereich der A. cerebri anterior findet sich kontralateral eine zuerst schlaffe, später spastische beinbetonte Hemiparese, oft einhergehend mit Sensibilitätsstörung und Blasenstörung. Ist das Stromgebiet der A. cerebri media betroffen, tritt häufig kontralateral eine schlaffe, brachiofazial betonte senso-motorische Hemiparese auf. Ist die dominante Hirnhemisphäre berührt, äußert sich dies zusätzlich in einer Aphasie des Patienten. Kontralaterale Hemihypästhesie und Hemianopsie liegen häufig bei Infarkten der A. cerebri posterior vor. Kleinhirninfarkte zeigen sich symptomatisch durch Koordinations-, Sprech- und Stimmstörungen. Bei ausgedehnten zerebralen Ischämien entwickelt sich infolge des zunehmenden Hirnödems eine Vigilanzstörung.

Die früher klassische Differenzierung anhand des Schweregrades und des zeitlichen Verlaufs der Symptome in transitorisch-ischämische Attacke (TIA), (prolongiertes) reversibles ischämisches neurologisches Defizit (RIND/PRIND), progressiver sowie vollendeter Infarkt gilt als überholt (AWMF-Leitlinien 2008). Sie wurde von der oben erwähnten pathophysiologischen Einteilung ersetzt, nachdem durch Fortschritte in der Bildgebung gezeigt werden konnte, dass auch bei vielen Patienten mit flüchtiger Symptomatik ( $<24$ h) morphologische Hirnschäden nachweisbar sind (Waxman und Toole 1983).

Eine TIA ist jedoch ein wichtiges Warnsignal für einen eventuell irreversiblen Schlaganfall, der auf die voll reversible Attacke in engem zeitlichen Zusammenhang folgen kann. Das Schlaganfall-Risiko ist besonders in den ersten Tagen nach einer TIA hoch. In 5,3\% der Fälle folgt auf eine TIA innerhalb von 2 Tagen ein vollendeter Schlaganfall, im 90-Tage-Verlauf bei insgesamt 10,5 \% der Patienten (Johnston et al. 
2000). Anhand des $A B C D^{2}$-Scores kann das Hirninfarktrisiko von TIA-Patienten allein auf der Basis einfacher Parameter (Alter, Blutdruck, Klinik, TIA-Dauer, Diabetes) zuverlässig stratifiziert werden (Giles und Rothwell 2010; Johnston et al. 2007).

\subsubsection{Bildgebung beim Schlaganfall}

Die neuroradiologische Bildgebung hat neben der klinisch-neurologischen Untersuchung den maßgeblichen Anteil an der Diagnostik des akuten Schlaganfalls. Sie liefert die Grundlagen für eine spezifische und damit effektive Therapie.

Dabei stehen verschiedene Verfahren mit jeweils unterschiedlichen Schwerpunkten zur Verfügung und sollen im Folgenden kurz in ihrer Bedeutung für Schlaganfalldiagnostik dargestellt werden.

Die bildgebende Diagnostik beim akuten Schlaganfall hat das Ziel, 4 Kernfragen zu beantworten (Trenkler 2008):

- Liegt eine intrakranielle Blutung vor?

- Besteht ein akuter Gefäßverschluss, der medikamentös oder mechanisch therapiert werden könnte?

- Ist ein (irreversibler) Infarktkern sichtbar?

- Besteht eine ischämische Penumbra (tissue at risk)?

\subsubsection{Pathophysiologie: Das Penumbra-Konzept}

Das Konzept der ischämischen Penumbra beschreibt in der neuroradiologischen Schlaganfall-Bildgebung das Hirnparenchym, welches durch Minderperfusion akut vital bedroht ist und durch schnelle therapeutische Maßnahmen noch vor der Infarzierung gerettet werden kann. Es ist definiert als Gewebe, mit gestörtem Funktionsstoffwechsel bei erhaltenem Strukturstoffwechsel infolge der Minderperfusion (Astrup et al. 1981).

Aufgrund des erhöhten Energiebedarfs ist der normale zerebrale Blutfluss (CBF) der grauen Substanz mit etwa $60 \mathrm{ml} / 100 \mathrm{~g}$ Hirngewebe/min um den Faktor 3 höher als bei der weißen Substanz mit etwa $20 \mathrm{ml} / 100 \mathrm{~g} / \mathrm{min}$. Bei einer Schwankung zwischen 60 und $20 \mathrm{ml} / 100 \mathrm{~g} / \mathrm{min}$ im Bereich der grauen Substanz, welche als benigne Oligämie bezeichnet wird, kommt es noch nicht zu klinischen Symptomen, da die zerebralen Blutgefäße zur Autoregulation befähigt sind. Das Gewebe ist zwar minderperfundiert, aber selbst bei persistierender Minderperfusion nicht unmittelbar vom Untergang bedroht. 


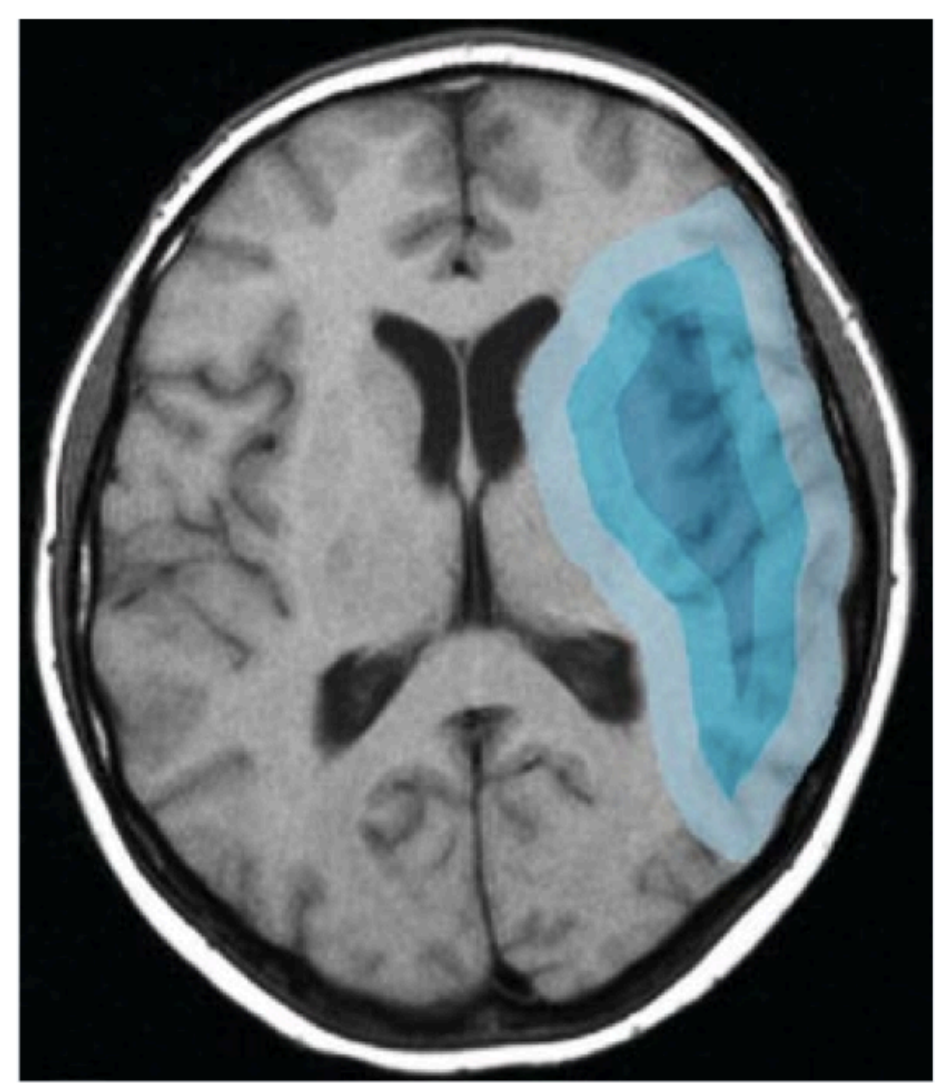

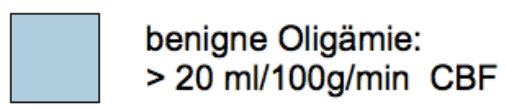

Penumbra:

$10-20 \mathrm{ml} / 100 \mathrm{~g} / \mathrm{min} \mathrm{CBF}$

Infarktkern:

$<10 \mathrm{ml} / 100 \mathrm{~g} / \mathrm{min} \mathrm{CBF}$

Sinkt der CBF unter einen Wert von $20 \mathrm{ml} / 100 \mathrm{~g} / \mathrm{min}$ (Ischämieschwelle), tritt eine transiente neuronale Funktionsstörung auf, die Zellstrukturen bleiben jedoch erhalten. Dieser "ischämische Penumbra“ oder tissue at risk genannte Bereich an Hirngewebe hat die Fähigkeit sich zu erholen, wenn die Perfusion rechtzeitig wieder hergestellt wird. Die Penumbra ist ein dynamisches, stark fluktuierendes Gewebeareal. Je länger der Zeitraum der kritischen Minderzirkulation fortbesteht, desto eher wird potentiell zu rettendes Gewebe doch infarziert. Die Ausdehnung wird bestimmt durch das Ausmaß der regionalen CBF-Minderung, durch den Ort des Gefäßverschlusses, den Status der Kollateralisierung und die zeitliche Dauer des Perfusionsdefizits.

Die ischämische Penumbra (aus dem Lateinischen: „Halbschatten“; paene (fast) \& umbra (Schatten)) wurde erstmals von Astrup et al. (1981) in der Literatur beschrieben und bekam den Namen in Anlehnung an die halbschattene Zone um das Zentrum einer totalen Sonnenfinsternis.

Bei einer weiteren Perfusionsminderung unter den kritischen Wert von 10 (- 15) $\mathrm{ml} / 100 \mathrm{~g} / \mathrm{min}$ (Infarktschwelle) über 2-4 Minuten bricht der Zellstoffwechsel zusammen, das Hirngewebe infarziert und ist damit irreversibel geschädigt (Powers et al. 1985). Diese Zone an nekrotischem Hirnparenchym wird Infarktkern genannt. Bei Patienten mit einem typischen Infarkt der großen hirnversorgenden Gefäße 
werden pro Minute 1,9 Millionen Neurone, 14 Milliarden Synapsen und $12 \mathrm{~km}$ myelinisierter Nervenfasern zerstört (Saver 2006).

Die Identifizierung und Differenzierung von Infarktkern und ischämischer Penumbra, welche potentiell zu retten ist, hat essentielle Bedeutung in der Akutdiagnostik als Entscheidungsgrundlage für das weitere therapeutische Vorgehen bei Schlaganfallpatienten (Paciaroni et al. 2009).

\subsubsection{Computertomographie (NECT, CTA, CTP)}

Die native Computertomographie (NECT) ist derzeit die Standardmethode der zerebralen Bildgebung beim akuten Schlaganfall und hat weltweit die größte Verfügbarkeit. Die entscheidende Bedeutung liegt im sicheren Ausschluss bzw. Nachweis intrakranieller Blutungen und ist somit laut nationalen und internationalen Leitlinienempfehlungen unbedingt erforderlich für die Indikationsstellung zur Thrombolyse (AWMF-Leitlinien 2008; European Stroke Organisation 2008; Quinn et al. 2009 b).

Neben dem Blutungs- und Neoplasieausschluss stehen bei der Beurteilung des NECT in der Schlaganfalldiagnostik die sogenannten "CCT-Frühzeichen“ (early ischemic signs) im Vordergrund, die differentialtherapeutische und prognostische Bedeutung haben (von Kummer et al. 1997). Zu diesen zählen die reduzierte RindenMark-Differenzierung („Frühhypodensität"), eine fokale Schwellung mit Verstreichen der Rindenfurchen, sowie ein hyperdenses Arterienzeichen, das den direkten Nachweis des Thrombus im Gefäß beschreibt; in den meisten Fällen in der A. cerebri media als hyperdenses Mediazeichen (dense media sign). Diese Frühzeichen sind in der hyperakuten Phase zwei bis sechs Stunden nach dem Infarktereignis nachzuweisen (Marks et al. 1999).

Die Sensitivität für den Ausschluss einer intrazerebralen Hämorrhagie ist mittels NECT sehr hoch (nahe $100 \%$ ), in einer Metaanalyse reichte die Sensitivität für Frühhypodensitäten von $20-87 \%$ mit einem Mittelwert von $66 \%$ (Wardlaw und Mielke 2005). Anhand des nativen CT alleine ist jedoch eine Identifizierung von Infarktkern und Penumbra in der Akutphase nicht möglich (Muir et al. 2007).

Die CT-Angiographie mittels Spiral-CT und Kontrastmittel (KM) stellt zusätzlich den gesamten extra- und intrakraniellen Verlauf der hirnversorgenden Arterien dar. Sie ist ein verlässliches Instrument für die Darstellung des Verschlussortes, der Länge des okkludierten Arteriensegmentes, eventuell vorhandener Kollateralkreisläufe, die das Infarktgebiet versorgen, sowie gegebenenfalls von Gefäßstenosen und Aneurysmen 
(Knauth et al. 1997). In der für die Lysetherapie (siehe Kapitel 1.3, Seite 24) interessanten hyperakuten Phase bis zu 6 Stunden nach Symptombeginn gelingt der CTA mit einer Spezifität und Sensitivität von jeweils etwa $98 \%$ der Nachweis bzw. Ausschluss eines Gefäßverschlusses (Lev et al. 2001). Eine Differenzierung von noch vitalem Risikogewebe und Infarktkern gelingt ebenfalls und zeigt eine hohe Korrelation mit Läsionen in diffusionsgewichteten MRT-Sequenzen (Schramm et al. 2004).

Während der Passage eines weiteren KM-Bolus können dynamische CT-Bilder erfasst werden (CT-Perfusion, CTP), aus denen funktionelle Parameterbilder des zerebralen Blutvolumens (CBV), zerebralen Blutflusses (CBF), der medianen KMTransitzeit (MTT) sowie der Zeit zum KM-Peak (TTP) berechnet werden können (Koenig et al. 2001).

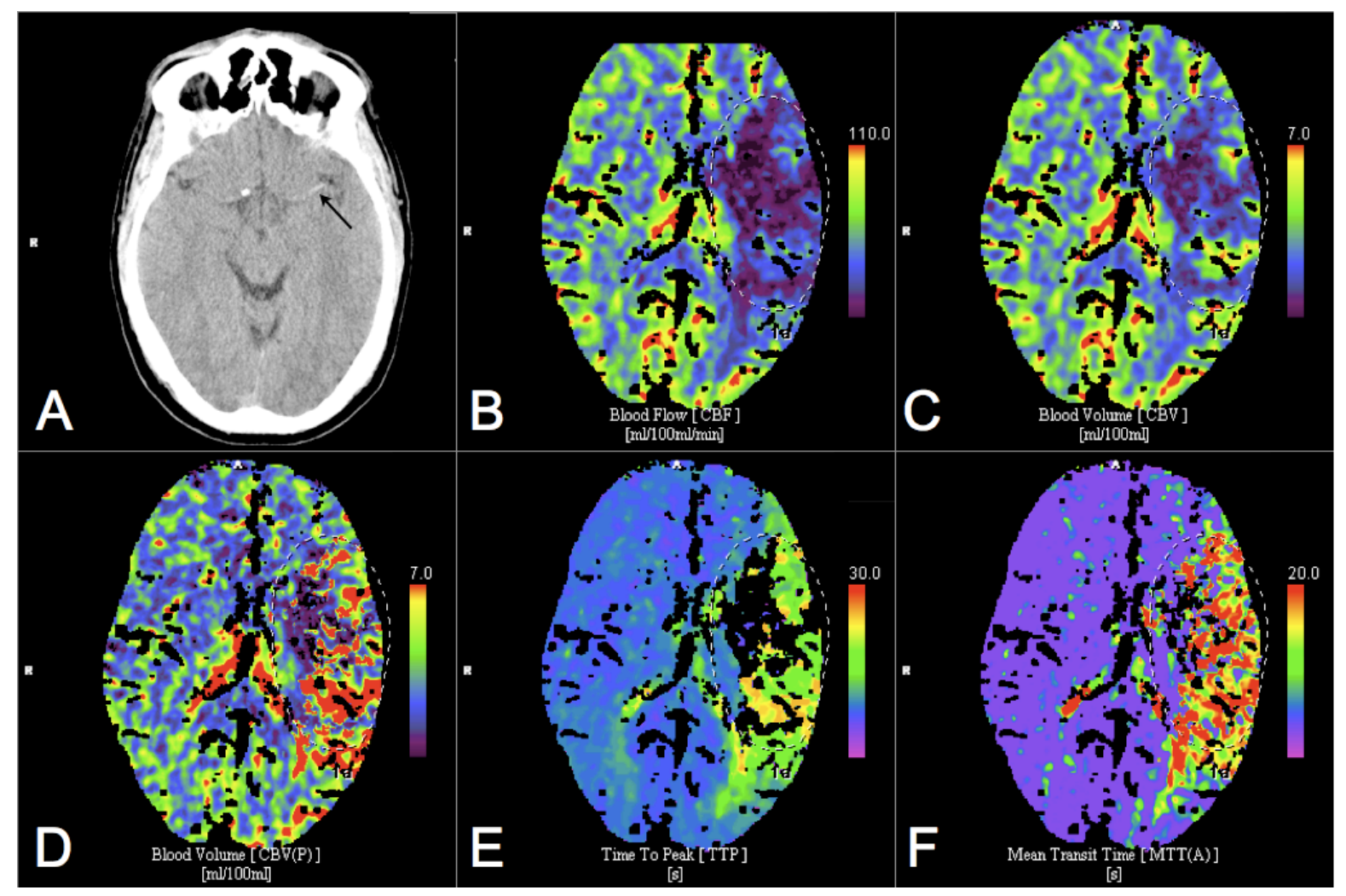

Abbildung 8 - Darstellung eines Schlaganfall-CT-Protokolls mit NECT (A) mit hyperdensem Media-Zeichen (siehe Pfeil), CBF (B), CBV (C und D), TTP (E) und MTT ( $F$ ) bei einem Patienten mit akutem Media-Verschluss links.

Diese Parameter erlauben eine sehr präzise Identifizierung des Infarktkerns und der Penumbra (siehe Abbildung 7, Seite 17). In der Akutphase des Schlaganfalls zeigt sich im Infarktkern ein CBF-CBV-Mismatch in der CTP mit einem CBV- 
Schwellenwert von $\leq 2,5 \mathrm{ml} / 100 \mathrm{~g}$. Währenddessen lässt sich im umliegenden Penumbra-Gewebe lediglich ein CBF-CBV-Mismatch mit normalem CBV $(>2,5 \mathrm{ml} /$ $100 \mathrm{~g}$ ) darstellen (Wintermark et al. 2002).

Durch die Festlegung der Grenzwerte von einer Reduktion $\geq 34 \%$ gegenüber dem CBF-Ausgangswert für die Penumbra und einem CBV von $\leq 2,5 \mathrm{ml} / 100 \mathrm{~g}$ für den Infarktkern konnten Wintermark et al. (2002) eine gute Korrelation zwischen dem MR-DWI und CT-CBV für den Infarktkern sowie zwischen dem MR-MTT und CT-CBF für die ischämische Penumbra nachweisen.

\subsubsection{Magnetresonanztomographie (MRT, MRA)}

Die Durchführung einer multiparametrischen Schlaganfall-MRT dient, wie die Schlaganfall-CT, zum Nachweis eines intra- oder extrakraniellen Gefäßverschlusses, zur Ermittlung des Infarktkernes und der von Infarzierung bedrohten Penumbra, sowie dem Ausschluss von intrazerebralen Blutungen oder anderen pathologischen Prozessen (siehe Abbildung 9, Seite 22).

Eine Schlaganfall-MRT-Untersuchung erlaubt die genaue Charakterisierung der Schlaganfall-Pathophysiologie und besteht aus einer Kombination verschiedener MRT-Sequenzen: einer diffusionsgewichteten Sequenz (diffusion weighted imaging, DWI), einer Perfusionsuntersuchung (perfusion weighted imaging, PWI), einer MR-Angiografie (MRA), einer blutungssensitiven Sequenz (z. B. einer T2*-gewichteten Gradientenechosequenz) sowie einer konventionellen fluid-attenuated inversion recovery (FLAIR) oder T2-gewichteten Sequenz zum Aufzeigen älterer Infarkte oder auch zum Tumorausschluss (Hjort et al. 2005).

Die wesentliche Stärke der MRT im Vergleich zur CT liegt in der deutlich höheren Sensitivität der DWI für die frühe ischämieinduzierte Verminderung der Wasserdiffusion (Fiebach et al. 2002; Saur et al. 2003), welche sich bereits innerhalb von Minuten nach Beginn der Ischämie nachweisen lässt (Moseley et al. 1990). Das Gewebe mit alleinig erhöhtem DWI-Signal bzw. mit Absenkungen des Apparent Diffusion Coefficient (ADC) infarziert meist, jedoch ist die eingeschränkte Diffusion nicht ganz spezifisch für eine ischämische Hirnschädigung. Das Ausmaß des hyperakuten Defektes, das aus den DWI- und ADC-Daten errechnet werden kann, korreliert mit dem Schweregrad des klinischen Befundes (Grant et al. 2001).

Die ischämische Penumbra kann erst unter Zuhilfenahme der PWI ermittelt werden. Für die Perfusionsuntersuchung mittels MRT wird in der klinischen Anwendung üblicherweise die Bolus-Track-Methode verwendet und die Anflutung eines 
paramagnetischen Kontrastmittels während der ersten Passage durch das Hirngewebe erfasst (Ostergaard 2005). Aus den registrierten SignalintensitätsZeit-Kurven können verschiedene Perfusionsparameter wie CBF, CBV, mittlere Transitzeit (mean transit time, MTT) oder die Zeit bis zum Maximum der Signalintensitäts-Zeit-Kurve (time to peak, TTP) berechnet werden.

DWI und PWI stellen nur annäherungsweise den Infarktkern (DWI) und das minderdurchblutete Hirnareal (PWI) dar. Das PWI-, aber nicht DWI-gestörte Hirnareal entspricht MR-morphologisch der ischämischen Penumbra (PWI/DWI-Mismatch) (Schlaug et al. 1999).

Aufgrund der Entwicklung neuer, schneller Techniken, wie das Echo-planar-imaging (EPI) für DWI-Sequenzen und Turbo- oder Fast-Spinecho-Sequenzen (TSE, FSE) für die T2W-Sequenzen, gewann die Schlaganfall-MRT durch Reduzierung der Akquisitionszeiten für DWI-Untersuchungen auf ca. 1-3 min / Sequenz deutlich an Bedeutung und hat nicht mehr den Nachteil eines großen Zeitverlustes für den Patienten.

Die MR-Angiografie (MRA) kann entweder mittels flusssensitiver Sequenzen (time of flight, TOF) oder über die Gabe von KM (contrast enhanced, CE-MRA) die extraund intrakraniellen hirnversorgenden Arterien darstellen und einen eventuellen Gefäßverschluss nachweisen (Muir et al. 2006). Die Stenosegrade werden eher überschätzt.

Nationale und internationale Leitlinien empfehlen die Bildgebung mit CCT oder MRT als gleichwertige Verfahren für den Nachweis akuter intrakranieller Blutungen. Die MRT kann in der Schlaganfall-Bildgebung die CCT ersetzen, wenn sie rasch zur Verfügung steht.

Wintermark et al. konnten durch den Vergleich von MRT und CTP/CTA zeigen, dass aus der Beurteilung der Verschusslokalisation, sowie der Infarktkern- und PenumbraGröße anhand dieser beiden Modaltäten sehr ähnliche Therapieentscheidungen getroffen würden bei Patienten mit akutem ischämischen Schlaganfall in einem Zeitfenster von 3-9 Stunden nach Symptombeginn (Wintermark et al. 2007). 


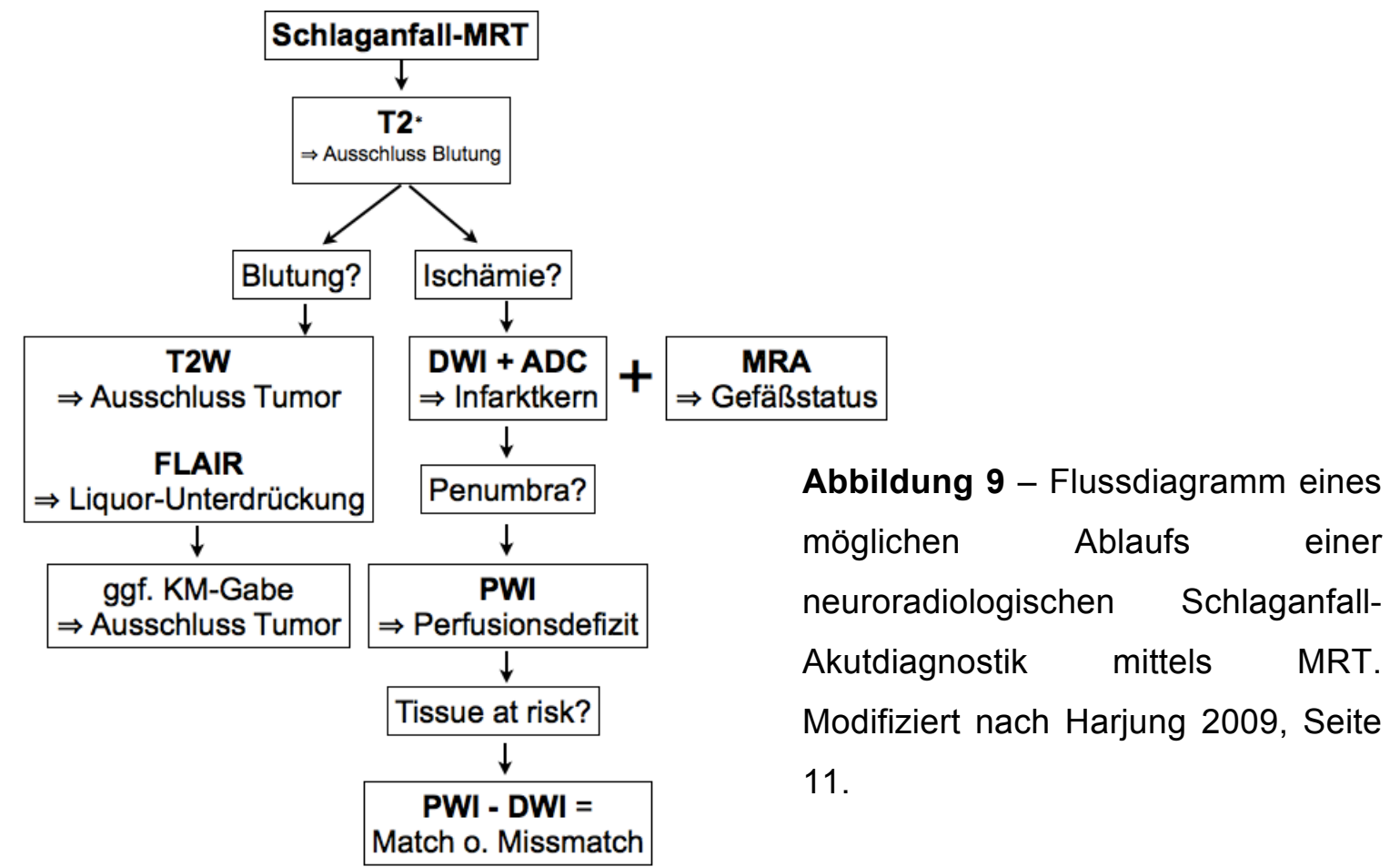

\subsubsection{Digitale Subtraktionsangiographie (DSA)}

Aufgrund der bisher unübertroffenen räumlichen und zeitlichen Auflösung stellt die digitale Subtraktionsangiographie (DSA) weiterhin den Goldstandard der neuroradiologischen Gefäßbildgebung dar (Higashida et al. 2003; Latchaw et al. 2009) (siehe Abbildung 14, Seite 50).

Für die meisten zerebrovaskulären Erkrankungen sind die Auflösung, die Sensitivität und die Spezifität in der Diagnostik durch die invasive DSA gleich- oder höherwertig als die zuvor beschriebenen nicht-invasiven Techniken. Lediglich die intrakraniellen Aneurysmen stellen eine Ausnahme dar: Hier ist die CTA der DSA gleichwertig oder besser bei der Diagnostik großer und in einigen Fällen sehr kleiner Aneurysmen, welche durch die DSA nicht immer sicher erkannt werden (Villablanca et al. 2000).

Die digitale Subtraktionsangiographie (DSA) hat inren Stellenwert im Zusammenhang mit der Möglichkeit einer direkten endovaskulären Behandlung, z.B. mechanischer Rekanalisation bei Verschlüssen hirnversorgender Gefäße, Stenting von Stenosen oder Coiling von Aneurysmen. Unverzichtbar ist die DSA für die präzise und selektive Diagnostik intrakranieller Gefäßstenosen, deren Grad, Länge und ggf. Kollateralisierung (Johnson et al. 2000; Rorick et al. 1994).

Mittels statischer Rekonstruktionen bietet die dreidimensionale Rotationsangiographie (3D-RA) gegenüber der 2D-DSA zusätzliche Informationen über den Gefäßstatus und ermöglicht eine bessere Beurteilung von Gefäßstenosen und -Läsionen (Anxionnat et al. 1998). 
Da es sich um ein invasives, Katheter-basiertes Verfahren der hirnversorgenden Gefäße handelt, können zwar seltene, dann jedoch schwerwiegendere Komplikationen auftreten. In der Literatur wird eine Komplikationsrate von $<1 \%$ bei zerebralen DSA-Prozeduren genannt (Willinsky et al. 2003), die weltweit größte Studie dazu berichtet von permanenten neurologischen Defiziten oder Todesfällen in $<0,2 \%$ der invasiven diagnostischen Angiographien (Kaufmann et al. 2007). Mögliche Komplikationen sind Thrombembolien, mit neurologischem Defizit, allergische Kontrastmittelreaktionen und ein postpunktionelles Leistenhämatom. Auch bei Patienten ohne scheinbare neurologische Komplikationen können sich nach durchgeführter DSA kleine asymptomatische Infarkte entwickeln, welche von Mikroemboli herrühren (Bendszus et al. 1999).

Für die allgemeine Notfalldiagnostik beim akuten Schlaganfall mit dem Ziel einer schnellen Darstellung der Gefäßsituation spielt die DSA deshalb im klinischen Alltag eine untergeordnete Rolle. Die auf der DSA basierenden interventionellen Therapieoptionen sind jedoch von zunehmender Bedeutung.

\subsubsection{Optionale Bildgebung}

Neurosonologische Methoden (extra- und transkranielle Duplexsonographie und Doppler-Sonographie, TCD) sind ein weiteres, klinisch wichtiges Werkzeug im Rahmen der neurologischen Akutdiagnostik beim Schlaganfall. Diese nicht-invasive Diagnostik extrakranieller und großer intrakranieller Gefäße ist Methode der Wahl zum Screening auf pathologische Veränderungen der extrakraniellen hirnversorgenden Arterien, erlaubt eine Darstellung der aktuellen Kollateralisation bei Gefäßverschlüssen und ermöglicht ein Monitoring der Dynamik von Gefäßveränderungen. Somit sind dynamische Prozesse während der Thrombolyse, wie Rekanalisation und Reokklusion, unmittelbar zu sehen und mit dem klinischen Status korrelierbar (Alexandrov et al. 2004).

Die neurosonologische Diagnostik sollte so früh wie möglich (innerhalb von 24 Stunden) nach Symptombeginn durchgeführt werden (AWMF-Leitlinien 2008).

Nuklearmedizinische Techniken wie die Positronenemissionstomografie (PET) und die Single-Photon-Emissions-Computertomografie (SPECT) spielen in der klinischen Routinediagnostik des Schlaganfalls in der Regel keine Rolle (Heiss und Podreka 1993; Thomalla et al. 2009). Ihre Bedeutung liegt vorwiegend im 
wissenschaftlichen Bereich, so konnten besonders PET-Untersuchungen wesentliche Erkenntnisse über die Pathophysiologie der zerebralen Ischämie liefern (Baron 1999). Die PET ist der Goldstandard für die Messung der zerebralen Perfusion und dient der Kalibrierung der Perfusions-Untersuchungen mit multiparametrischer CT und MRT (Takasawa et al. 2008).

Weitere klinisch unbedeutende Modalitäten sind das Xenon-enhanced CT (Xe-CT) und die MR-Spektroskopie (MRS) (Latchaw 2004).

\subsection{Therapien des Schlaganfalls}

Der Schlaganfall ist ein akuter medizinischer Notfall, der aufgrund eines zeitlich limitierten Handlungsrahmens beispielsweise für eine Lysetherapie eine schnelle Therapie ohne Verzögerung in spezialisierten Schlaganfall-Zentren (Stroke-Units) benötigt. Die schnelle Aufnahme auf eine multidisziplinäre Spezialstation für Schlaganfall-Patienten verbessert die Prognose des Patienten hinsichtlich Mortalität, klinischen Outcomes und Pflegeabhängigkeit; eine Reduktion der Mortalität relativ um 18-46 \% (absolut $3 \%$ ) wird erreicht (Stroke Unit Trialists' Collaboration 2007). Zudem bedarf es einer öffentlichen Aufklärung, um die Bevölkerung über das Krankheitsbild zu informieren sowie den lebensbedrohlichen Notfallcharakter des Schlaganfalls zu betonen (Brice et al. 2002).

Für die Therapie gilt immer der Grundsatz: Time is brain! (Saver 2006)

Die schnelle Rekanalisation des verschlossenen intrakraniellen Gefäßes stellt bei allen im Folgenden vorgestellten Therapien den wichtigsten Ansatz zur Akuttherapie des ischämischen Schlaganfalls dar. Nur die Reperfusion kann ischämisches, von Infarzierung bedrohtes Hirngewebe retten und irreversible neurologische Ausfälle verhindern.

Die internistische und neurologische Diagnostik und Therapie bilden, neben der neuroradiologischen Bildgebung, die Basis für weiterführende Therapien.

Entsprechend den aktuellen Leitlinien der Deutschen Gesellschaft für Neurologie und der Deutschen Schlaganfallgesellschaft (AWMF-Leitlinien 2008) setzt sich die medizinische Behandlung eines Schlaganfall-Patienten aus 5 Bestandteilen zusammen:

1. Intensivmedizinische Behandlung

2. Spezifische Akuttherapie (intravenöse \& intraarterielle Verfahren) 
3. Frühe Sekundärprophylaxe

4. Vorbeugung und Behandlung von Komplikationen

5. Frühe Rehabilitation.

\subsubsection{Notfallmedizinische Behandlung}

Die adäquate Behandlung und Erhaltung der Vitalfunktionen sowie die Behandlung entgleister physiologischer Parameter bilden die Basis der Schlaganfallbehandlung, bevor mit der spezifischen Behandlung des Schlaganfalls begonnen werden kann. Das adäquate Management der Vitalparameter soll schnell und ohne wesentliche Verzögerung der spezifischen Therapie erfolgen. Es fehlen randomisierte Interventionsstudien, aber pathophysiologisch gesehen sind sie sinnvoll, um weiteren Komplikationen vorzubeugen.

Der neurologische Status und die Vitalparameter sollen konstant überwacht werden.

\begin{tabular}{|c|c|c|}
\hline \multicolumn{3}{|c|}{ Notfallmedizinische Behandlung der Vitalfunktionen beim Schlaganfall } \\
\hline System & Therapie & Effekt \\
\hline \multirow{4}{*}{ Systemisch } & Körpertemperatur: Senkung ab $37,5^{\circ} \mathrm{C}$ & Prognose $\uparrow$ \\
\hline & $\begin{array}{l}\text { Flüssigkeits- und Elektrolythaushalt: Kontrolle und } \\
\text { Substitution }\end{array}$ & Entgleisungen $\downarrow$ \\
\hline & Insulintherapie ab Serumglukosespiegel $>200 \mathrm{mg} / \mathrm{dl}$ & Infarktprogress $\downarrow$ \\
\hline & $\begin{array}{l}\text { Thromboseprophylaxe (Heparin, Heparin o. } \\
\text { Stützstrümpfe), wenn eine Emboliequelle gesichert } \\
\text { oder wahrscheinlich ist }\end{array}$ & $\begin{array}{l}\text { Thrombosen } u \text {. } \\
\text { Lungenembolien } \downarrow\end{array}$ \\
\hline \multirow[b]{2}{*}{$\underline{\text { Kardial }}$} & Behandlung von Herzrhythmusstörungen & Emboliequelle $\downarrow$ \\
\hline & $\begin{array}{l}\text { Vermeidung starker Blutdruckschwankungen während } \\
\text { der Akutphase, Einstellung der Blutdruck-Zielwerte: } \\
\text { - vorbestehender Normotonus: } \\
160-180 \mathrm{mmHg} \text { systolisch } \\
90-100 \mathrm{mmHg} \text { diastolisch } \\
\text { - vorbestehender Hypertonus: } \\
180 \mathrm{mmHg} \text { systolisch } \\
100-105 \mathrm{mmHg} \text { diastolisch } \\
\text { Zentraler Venendruck etwa } 8-10 \mathrm{~cm} \mathrm{H}_{2} \mathrm{O}\end{array}$ & $\begin{array}{l}\text { Penumbra- } \\
\text { Perfusion } \uparrow\end{array}$ \\
\hline Pulmonal & Freihaltung der Atemwege, eventuell Oxygenierung & $\begin{array}{l}\text { Penumbra- } \\
\text { Oxygenierung } \uparrow\end{array}$ \\
\hline
\end{tabular}

Tabelle 1 - Übersicht über das notfallmedizinische Management der Vitalparameter beim ischämischen Schlaganfall. 


\subsubsection{Intravenöse Thrombolyse}

Die bisher einzige zugelassene spezifische Therapie des akuten Schlaganfalls ist die intravenöse Thrombolyse (IVT) mit rekombinantem gewebespezifischen Plasminogenaktivator (recombinant tissue-type plasminogen activator, rt-PA) innerhalb von 3 Stunden nach Symptombeginn (Köhrmann 2010). Die IVT wird von den meisten nationalen und internationalen Schlaganfall-Gesellschaften als first-lineTherapeutikum bei ischämischem Schlaganfall empfohlen (Adams et al. 2007; AWMF-Leitlinien 2008; European Stroke Organisation 2008).

Das gentechnisch hergestellte indirekte Fibrinolytikum rt-PA (Freiname Alteplase, Handelsname Actilyse) mit einer Plasma-Halbwertzeit von 3 - 8 Minuten imitiert die Wirkung des natürlichen gewebespezifischen Plasminogenaktivators, indem es über eine verstärkte Plasminsynthese eine Auflösung der Fibrin-Polymere des Thrombus induziert.

Nach Ausschluss einer intrazerebralen Blutung und einer großen ischämischen Infarktausdehnung mittels CT oder MRT wird rt-PA in einer Dosis von $0,9 \mathrm{mg} / \mathrm{kg} \mathrm{KG}$ (Maximum von $90 \mathrm{mg}, 10 \%$ der Gesamtdosis als Bolus, die restlichen $90 \% \mathrm{im}$ Anschluss als Infusion über 60 Minuten) intravenös apliziert.

Die Zulassung der IVT erfolgte in Europa im Jahr 2002 auf Grundlage der 1995 publizierten amerikanischen National Institute of Neurological Disorders and Stroke (NINDS) Study (NINDS rt-PA Stroke Study Group 1995). Sie erbrachte den Nachweis der Effektivität der IVT in der Behandlung des akuten ischämischen Schlaganfalls im Zeitfenster von 3 Stunden nach Symptombeginn. Im Vergleich zur Placebogruppe hatten Patienten, die mit rt-PA behandelt wurden, eine mindestens $30 \%$ höhere Wahrscheinlichkeit, nur minimale oder keine Behinderungen 3 Monate nach dem Schlaganfallsereignis zu haben.

Zahlreiche, meist randomisierte und kontrollierte Studien (ATLANTIS, ECASS I, ECASS II, SITS-MOST; siehe Tabelle 4, Seite 36/37) konnten den therapeutischen Nutzen und die Sicherheit der IVT in den Folgejahren bestätigen (Clark et al. 1999; Hacke et al. 1995; Hacke et al. 1998; Wahlgren et al. 2007). Hacke et al. konnten durch die europäische ECASS-III-Studie (European Cooperative Stroke Study) auch eine Wirksamkeit der IVT im 3- bis 4,5-h-Zeitfenster nachweisen, ohne ein höheres Risko für eine intrakranielle Blutung im Vergleich zum 3-Stunden-Zeitfenster eingehen zu müssen (Hacke et al. 2008). Allerdings hat dies bisher noch nicht zu 
einer Erweiterung der Zulassung geführt, womit die CT-basierte IVT im 3- bis 4,5-h-Zeitfenster nach entsprechender Aufklärung nur "off-label“ anwendbar ist.

Medikamentöse Lysen intrazerebraler Gefäße mehr als 3 Stunden nach Symptombeginn werden in Deutschland als individueller Heilversuch gewertet und gehen mit einem erhöhten Komplikationsrisiko einher (AWMF-Leitlinien 2008).

\begin{tabular}{|c|c|}
\hline \multicolumn{2}{|l|}{ IVT-Zulassungsstudien } \\
\hline Einschlusskriterien & Ausschlusskriterien \\
\hline $\begin{array}{l}\text { Klinische Diagnose eines ischämischen } \\
\text { Schlaganfalls }\end{array}$ & Orale Antikoagulanzientherapie \\
\hline Alter 18 - 80 Jahre & kurz zurückliegende schwere Blutung \\
\hline \multirow{2}{*}{$\begin{array}{l}<3 \mathrm{~h} \text { seit Symptombeginn bzw. }<4,5 \mathrm{~h} \\
\text { nach ECASS-III, dann aber "off-label“ } \\
\text { Intrakranielle Blutung mittels Bildgebung } \\
\text { ausgeschlossen }\end{array}$} & $\begin{array}{l}\text { Verdacht auf oder Zustand nach } \\
\text { Subarachnoidalblutung }\end{array}$ \\
\hline & $\begin{array}{l}\text { bekannte intrakranielle Neoplasie oder } \\
\text { unversorgtes Aneurysma }\end{array}$ \\
\hline \multirow[t]{5}{*}{$\begin{array}{c}\text { Überwachung auf Intensiv-Station oder } \\
\text { Stroke-Unit }\end{array}$} & $\begin{array}{l}\text { kurz zurückliegende Punktion eines nicht } \\
\text { komprimierbaren Gefäßes }\end{array}$ \\
\hline & unkontrollierbare schwere Hypertonie \\
\hline & $\begin{array}{l}\text { größere Operationen oder Traumata in den } \\
\text { letzten } 3 \text { Monaten }\end{array}$ \\
\hline & $\begin{array}{l}\text { schwere Lebererkrankung, gastrointestinale } \\
\text { Blutung }\end{array}$ \\
\hline & $\begin{array}{l}\text { bekannte Neoplasie mit erhöhter } \\
\text { Blutungsneigung }\end{array}$ \\
\hline
\end{tabular}

Tabelle 2 - Wichtigste Ein- und Ausschlusskriterien der Zulassungsstudien für die IVT (Hacke et al. 2008; Köhrmann 2010).

Bei der IVT besteht ein zeitabhängiger Behandlungseffekt. Je früher rt-PA beim akuten Hirninfarkt gegeben wird, desto größer ist der therapeutische Benefit. Insbesondere, wenn innerhalb von 90 min nach Symptombeginn therapiert wird, ist die Chance darauf, ein exzellentes Rehabilitationsergebnis ohne verbleibende Behinderungen zu erzielen, 2,8-fach gegenüber Placebo erhöht, innerhalb von 91180 min nur noch 1,6-fach und von 181-270 min ca. 1,4-fach (Hacke et al. 2004).

Aktuellere Studien deuten eine höhere Sicherheit und möglicherweise einen gesteigerten Vorteil für individuelle Patienten an, die später als $3 \mathrm{~h}$ nach Symptombeginn mittels MRT-basierter Selektionskriterien anhand des PWI/DWIMismatch-Prinzips mit rt-PA behandelt wurden (Köhrmann et al. 2006; Schellinger et al. 2007). Die prospektive, nicht-randomisierte DEFUSE-Studie konnte bei Patienten, die ein deutliches MRT-basiertes DWI/PWI-Mismatch (DWI $<100 \mathrm{ml}$ und $<80 \%$ des 
PWI; PWI >10 ml) hatten, bei früher Reperfusion 3 - 6 Stunden nach Symptombeginn durch IVT mittels rt-PA einen klinischen Nutzen aufzeigen (Albers et al. 2006).

Die größte Gefahr im Rahmen einer IVT sind intrakranielle Hämorrhagien (ICH) durch im Infarktgebiet vorgeschädigte Gefäße. In der Literatur wird die Inzidenz für symptomatische ICH bei IVT mit 2,4 - 11,4\% angegeben (Placebo-Kontrollgruppe $0,2-4,7 \%$ ), bei rt-PA-Gebrauch liegt das Odds Ratio bei 3,28 im Vergleich zur Placebo-Kontrollgruppe (Wardlaw et al. 2009).

Aufgrund der mit rt-PA assoziierten Nebenwirkungen wurden zahlreiche Anstrengungen unternommen, alternative Fibrinolytika mit längerer Halbwertszeit, höherer Fibrin-Spezifität, niedrigeren $\mathrm{ICH}$-Raten und besseren therapeutischen Effekten zu finden, aber bisher konnte kein anderes Fibrinolytikum, wie z.B. Urokinase, Tenecteplase, Desmoteplase oder Ancrod, seine Überlegenheit gegenüber rt-PA beweisen.

\subsubsection{Intraarterielle Rekanalisationsverfahren}

Neben der IVT gibt es eine Vielzahl invasiver Rekanalisationstherapien, welche auch mit der IVT kombiniert werden können und als individueller Heilversuch bei bestimmten Patientengruppen mit akutem ischämischen Schlaganfall eingesetzt werden können. Diese intraarteriellen, sowie besonders die mechanischen, endovaskulären Therapien sind allerdings spezialisierten, interventionellneuroradiologischen Zentren vorbehalten.

\subsubsection{Intraarterielle Thrombolyse}

Das Ziel der intraarteriellen Thrombolyse (IAT) ist die Gefäßrekanalisation durch Infusion eines Fibrinolytikums mittels Katheter direkt an den Ort des Gefäßverschlusses. Die IAT hat den Vorteil einer höheren lokalen Konzentration des Fibrinolytikums bei gleichzeitiger Reduzierung der benötigten rt-PA-Gesamtdosis, was zu einer Minimierung der systemischen Wirkung führt. Die IAT ist somit potenziell effektiver und sicherer als die IVT (Köhrmann 2010). Aus diesen Gründen kann der Therapiezeitrahmen über die für IVT festgelegten 3 bzw. 4,5 Stunden ausgedehnt werden. Dieser Faktor wird besonders wichtig angesichts der relativ geringen Anzahl von Patienten, die innerhalb des IVT-Zeitfensters therapiert werden kann (Albers und Olivot 2007).

Diese invasive Therapieoption bietet den Vorteil, dass vor oder nach erfolgreicher Gefäßwiedereröffnung eine dem Verschluss ggf. zugrunde liegende Stenose mittels 
perkutaner transluminaler Angioplastie und evtl. Stenting behandelt werden kann und so die Wahrscheinlichkeit einer Reokklusion verringert. Nachteilig können der technisch hohe Aufwand, der damit einhergehende Zeitverlust bis zum Therapiebeginn, Risiken der invasiven Prozedur und die relativ geringe Verfügbarkeit sein.

Die IAT erzielt im Vergleich mit der IVT bei Patienten mit akutem ischämischen Schlaganfall höhere Rekanalisationsraten (Mattle et al. 2008). Der therapeutische Benefit ist bei gleichzeitig reduzierter Mortalität größer, dagegen steigt die Inzidenz symptomatischer ICH (Lisboa et al. 2002). Die IAT bei Patienten mit Verschlüssen der proximalen A. cerebri media mit Pro-Urokinase innerhalb von $6 \mathrm{~h}$ führte zu einem signifikant besseren Outcome in der PROACT II-Studie (Furlan et al. 1999). Weitere Vergleichs- und Beobachtungsstudien (PROACT I, MELT; siehe Tabelle 4, Seite 36/37) bestätigen ebenfalls den Nutzen der IAT bei M1-Verschlüssen (del Zoppo et al. 1998; Ogawa et al. 2007).

Die intraarterielle Behandlung von Basilarisverschlüssen mit Urokinase oder rtPA wird ebenfalls mit Erfolg an spezialisierten, interventionell-angiographisch tätigen Zentren eingesetzt und in einer kleinen randomisierten Studie wurden gute Ergebnisse im hinteren arteriellen Kreislauf erzielt (Macleod et al. 2005). Eine systematische Analyse fand allerdings keinen Unterschied zwischen intravenöser und intraarterieller Thrombolyse bei Patienten mit Basilarisverschluss (Lindsberg und Mattle 2006).

\subsubsection{Mechanische Rekanalisation}

Die einfachste Form der mechanischen Rekanalisation besteht aus der Passage des Thrombus mit einem Mikrodraht während der IAT, sodass durch mechanische Thrombusfragmentierung die Thrombusoberfläche vergrößert wird und die Angriffsfläche für das Fibrinolytikum erhöht wird (augmented fibrinolysis) (Saver 2011).

Neben der medikamentösen Thrombolyse mit rt-PA kann der Thrombus ebenfalls durch verschiedene intraarterielle, rein mechanische Verfahren aus der Hirnarterie entfernt werden. Dazu steht eine Vielzahl neuartiger mechanischer Rekanalisationsverfahren zur Verfügung, welche hohe Rekanalisationsraten erzielen (siehe Abbildung 10, Seite 32). Sie können entsprechend ihrem Ansatzort im Gefäß für die Therapie in 3 verschiedene Gruppen unterteilt werden (Kreusch und Knauth 2011): 
1. proximale Systeme: Sie versuchen eine Thrombusentfernung von einer proximalen Plazierung des Instrumentes vor dem Thrombus aus

z.B. Penumbra System ${ }^{\mathrm{TM}}$, Alligator ${ }^{\mathrm{TM}}$, AngioJet ${ }^{\mathrm{TM}}, \mathrm{EPAR}^{\mathrm{TM}}$

2. distale Systeme: Ihnen gemeinsam ist der Ansatzpunkt hinter dem Thrombus, d.h. das System muss erst durch den Thrombus hindurch geführt und hinter inm platziert werden

z.B. MERCl ${ }^{\mathrm{TM}}$, Catch $^{\mathrm{TM}}$, Phenox $\mathrm{pCR} / \mathrm{CRC}{ }^{\mathrm{TM}}$, Lazarus ${ }^{\mathrm{TM}}$, Neuronet ${ }^{\mathrm{TM}}$, Snare ${ }^{\mathrm{TM}}$

3. „on the spot"-Systeme: Diese Verfahren setzen direkt im Thrombus an

z.B. EKOS ${ }^{\mathrm{TM}}$, Stents, Stent-artige Stentriever wie Solitaire ${ }^{\mathrm{TM}}$, BONnet ${ }^{\mathrm{TM}}$, Trevo $^{\mathrm{TM}}$, Mindframe ${ }^{\mathrm{TM}}$, ReVive $^{\mathrm{TM}}$

Die mechanische Rekanalisation kann drei Grundprinzipien folgen: Thrombusaspiration, Thrombusextraktion und Thrombusfrakturierung.

Die Zerkleinerung des Thrombus durch mechanische Manipulation begünstigt eine Rekanalisierung des verschlossenen Gefäßes, erhöht aber gleichzeitig auch die Gefahr peripherer Thrombembolien.

Als erstes Instrument bekam der Korkenzieher-ähnliche $\mathrm{MERCl}$ Retriever ${ }^{\mathrm{TM}}$ (Concentric Medical Inc., Mountain View, CA, USA) die Zulassung der Food and Drug Association (FDA) für die Thrombusentfernung aus Hirnarterien, wobei dieser distal des Thrombus ansetzt (siehe Abbildung $10 \mathrm{~B}$, Seite 32). Ausdrücklich erfolgte jedoch keine Zulassung als therapeutisches Verfahren, da keine Klarheit darüber bestand, ob die mechanische Thrombektomie bei akutem ischämischen Schlaganfall das klinische Endergebnis der Patienten verbessert. Nichtsdestotrotz wird die Thrombektomie im klinischen Alltag vielfach angewendet bei Patienten, die eine Kontraindikation für IVT haben oder wo diese Therapie nicht erfolgreich war. Die Studien MERCI I und II (siehe Tabelle 4, Seite 36/37) beschrieben hohe Rekanalisationsraten von bis zu $55 \%$ durch rein mechanische Rekanalisation mit dem MERCI Retriever und bis $68 \%$ bei zusätzlicher IAT, ebenso konnte eine signifikante Korrelation zwischen erfolgreicher Rekanalisation und gutem klinischem Endergebnis gezeigt werden (Gobin et al. 2004; Smith et al. 2008).

Das Penumbra System ${ }^{\mathrm{TM}}$ (Penumbra Inc., Alameda, CA, USA) greift proximal am Thrombus an, besteht aus einem Reperfusionskatheter für die kontinuierliche Aspirationsthrombektomie und einem Separator, der ein Verstopfen des Katheters 
verhindert (siehe Abbildungen $10 \mathrm{C}, 16,17,18$, sowie Tabelle 5, Seiten 52-55). In den ersten Studien konnten sehr hohe Rekanalisationsraten von $100 \%$ und 81,6 \% erzielt werden (Bose et al. 2008; Penumbra Pivotal Stroke Trial Investigators 2009), die FDA-Zulassung erfolgte 2007. Trotz hoher Gefäßeröffnungsraten hatten im Penumbra Pivotal Stroke Trial nur $25 \%$ der Patienten ein gutes klinisches Endergebnis nach 90 Tagen, die $\mathrm{SICH}-$ Raten waren vergleichbar mit dem MERCI Retriever (11,2 vs. 9,8\%) Zahlreiche weitere kleinere Fallserien belegten in der Folge die hohen Rekanalisationsraten (siehe Tabelle 4, Seite 36/37) und einzelne Einzelfallberichte berichten vom Einsatz des Penumbra Systems auch für die mechanische Rekanalisation von Dura-Sinus-Thrombosen (Blackham 2011; Choulakian und Alexander 2010; Kulcsar et al. 2010 b).

Das Catch Retrieval System mit einem selbstexpandierenden Fangkörbchen (Balt, Montmorency, Frankreich) wurde in einer größeren Fallserie getestet, jedoch blieb die Rekanalisationserfolgsrate mit $70 \%$ hinter den zuvor beschreibenen Systemen zurück und die SICH-Rate war mit $18 \%$ hoch (Mourand et al. 2011).

Die beiden Bürsten-ähnlichen Phenox-Systeme pCR und CRC (siehe Abbildung 10 A) mit flexiblen Mikrofilamenten oder das selbstexpandierbare Nitinoldrahtgeflecht BONnet (Phenox $\mathrm{GmbH}$, Bochum, Deutschland) wurden bisher nicht in größeren Patientenkollektiven getestet (Henkes et al. 2006). Auch experimentelle Ansätze mit Schlingen-ähnlichen Instrumenten (Neuronet ${ }^{\mathrm{TM}}$, Snare ${ }^{\mathrm{TM}}$ ) wurden nie in größeren Fallserien bewertet.

$\mathrm{Zu}$ den neuesten Entwicklungen zählen die sogenannten Stentriever, eine Kombination aus selbstexpandierendem Stent und Retriever (siehe Abbildung 10 D). Der Stent wird direkt im Thrombus freigesetzt, drückt diesen an die Gefäßwand und die Stent-Streben umschlingen den Thrombus. Anschließend kann der Stentriever mitsamt dem umwickelten Thrombus zurückgezogen werden. Auch die Begriffe RSAR (Removable Stent-assisted Revascularization) und flow restauration device finden Verwendung in der Literatur.

Gegenüber den anderen Thrombektomie-Instrumenten werden folgende Vorteile als vielversprechend angesehen: 1. Sofortige Flusswiederherstellung nach StentrieverFreisetzung („temporärer endovaskulärer Bypass“), 2. höhere Rekanalisationsraten als mit anderen Thrombektomie-Systemen und 3. die Option, den Stent am 
Verschlussort zu implantieren, falls der Thrombus nicht extrahiert werden kann (Saver 2011).

Erste publizierte Fallserien mit den Stentrievern Solitaire ${ }^{\mathrm{TM}}$, Trevo ${ }^{\mathrm{TM}}$ und ReVive ${ }^{\mathrm{TM}}$ belegen sehr hohe Rekanalisationsraten von 89 - $100 \%$ und zugleich gute funktionelle Endergebnisse bei bis zu 46 \% der Patienten nach 90 Tagen (Castano et al. 2010; Machi et al. 2011; Rohde et al. 2011).

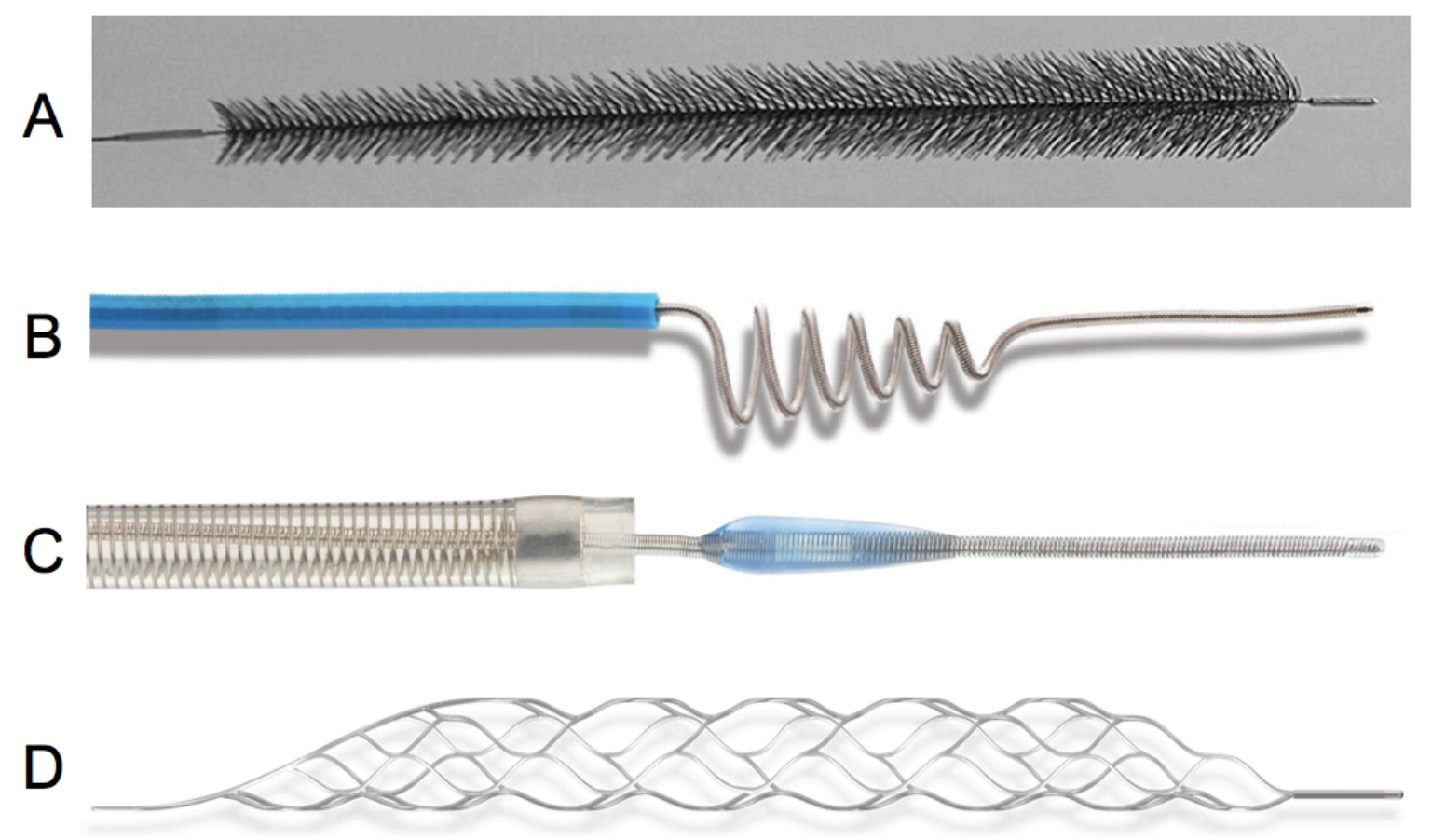

Abbildung 10 - Übersicht über verschiedene Instrumente zur mechanischen Rekanalisation:

A Phenox pCR, mit freundlicher Abdruckgenehmigung von Phenox $\mathrm{GmbH}$, Bochum

B MERCI Retriever X6, mit freundlicher Abdruckgenehmigung von Concentric Medical Inc., Mountain View, CA, USA

C Penumbra System, mit freundlicher Abdruckgenehmigung von Penumbra Inc., Alameda, CA, USA

D Trevo Pro 4 Stentriever, mit freundlicher Abdruckgenehmigung von Concentric Medical Inc., Mountain View, CA, USA.

Die perkutane transluminale Angioplastie kann auch zu hohen Rekanalisationsraten führen, in einer Metastudie zu 84 \% (Rha und Saver 2007), und findet Berechtigung bei in situ atherosklerotischen Plaques mit zusätzlichem thrombotischen Verschluss, aber es besteht eine erhöhte Gefäßrupturgefahr und eine hohe Rate an ReVerschlüssen. 
Eine weitere Möglichkeit der Rekanalisation ist die direkte Implantation eines Stents an den Ort des Verschlusses, sodass der Thrombus an die Gefäßwand gedrückt und eine sofortige Flusswiederherstellung erzielt werden kann. Es werden schnelle und hohe Rekanalisationsraten erzielt, aber zu den Problemen zählt die schwierige Navigation eines Stents in die gewundenen Hirngefäße, die gegebenenfalls unnötige Implantation eines Fremdkörpers, das damit einhergehende Risiko einer In-StentThrombose und -Stenose sowie die bei Stentimplantation notwendige duale Thrombozytenaggregationshemmung (Brekenfeld et al. 2009; Levy et al. 2009). In der extrakraniellen Zirkulation hat das notfallmäßige Stenting von akuten $\mathrm{ACl}-$ Verschlüssen weiterhin eine Bedeutung, ebenso intrakraniell als Notlösung beim Versagen anderer Thrombektomieverfahren.

\subsubsection{Sonothrombolyse}

Seit einigen Jahren wird die Idee der Sonothrombolyse verfolgt. Niedrigfrequenter transkranieller Doppler-Ultraschall (2 Mhz) kann die enzymatische Thrombolyse mit rt-PA verstärken, indem die Thrombus-Permeabilität durch den Schalldruckgradienten herabgesetzt wird und das Fibrinolytikum besser in das Thrombusmaterial eindringen kann. Der EKOS Mikro-Infusionskatheter (EKOS Corporation, Bothell, WA, USA) ist ein kombinierter Infusions- und Ultraschallkatheter mit einer Ultraschallsonde an der Katheterspitze. In der CLOTBUST- (iv rt-PA) und der IMS IIStudie (ia rt-PA) wurde die Überlegenheit der Sonothrombolyse gegenüber IVT bestätigt, die Rekanalisationsarten lagen bei $49 \%$ bzw. $73 \%$ mit einem Trend zu einem verbesserten 90-Tage-Outcome gegenüber IVT (siehe Tabelle 4, Seite 36/37) (Alexandrov et al. 2004; IMS II Trial Investigators 2007).

Die Effizienz der Sonothrombolyse kann durch die Kombination der UltraschallApplikation mit einem Ultraschallkontrastmittel, das aus gashaltigen Mikrosphären besteht, gesteigert werden (Molina et al. 2006).

\subsubsection{Kombinationstherapien}

Die intravenösen und intraarteriellen Rekanalisationsverfahren können als einzelne therapeutische Akut-Maßnahmen eingesetzt werden oder in bestimmten Kombinationen zum Einsatz kommen, um die Effektivität bezüglich Gefäßwiedereröffnung und Reperfusion zu maximieren (siehe Tabelle 3, Seite 35). 
Die Kombination aus initialer IVT und anschließender endovaskulärer Thrombolyse/Thrombektomie wird „Bridging-Konzept“ genannt. Dabei wird die Zeit bis zur invasiven Therapie durch eine frühzeitige IVT „überbrückt“ und der Thrombus für eine erleichterte endovaskuläre Therapie „angedaut“. Eine kombinierte intravenöse und intraarterielle Therapie bietet die Vorteile eines schnellen Therapiestarts durch IVT und hoher Rekanalisationsraten durch endovaskuläre Therapien. Im Gegensatz zu diesen additiven Effekten scheinen die Nebenwirkungen der einzelnen Therapieansätze sich nicht $\mathrm{zu}$ summieren. In mehreren Kohortenstudien konnte gezeigt werden, dass das Hämorrhagie-Risiko der kombinierten Therapie nicht wesentlich höher ist als das Blutungsrisiko der alleinigen endovaskulären Behandlung (Saver 2011).

Die RECANALISE-Studie verglich den kombinierten IVT-mechanischen Therapieweg mit der reinen IVT durch rt-PA und fand bei der kombinierten Therapie eine höhere Rekanalisationsrate (87 vs. $52 \%$ ) und auch eine höhere Rate von gutem klinischen Endergebnis (57 vs. 44 \%) (Mazighi et al. 2009). Eindeutige Ergebnisse werden von der randomisierten, multizentrischen IMS-III-Studie (Interventional Management of Stroke III) erwartet, welche IVT versus IVT + IAT/Thrombektomie (Bridging-Konzept) testet (siehe Tabelle 8, Seite 98) (Khatri et al. 2008).

Die mechanische Gefäßrekakalisation kann ebenfalls durch rt-PA-Gabe während der intraarteriellen Intervention unterstützt werden, dies kann die Thrombusentfernung erleichtern und das Auftreten von distalen Embolien aufgrund von Thrombusfragmentation während mechanischer Rekanalisation minimieren.

Bei allen Kombinationstherapien sollte das Gesamtdosislimit für rt-PA von 0.9 mg rt$\mathrm{PA} / \mathrm{kg}$ Körpergewicht eingehalten werden, jedoch deuten Veröffentlichungen darauf hin, dass der intraarterielle Einsatz von rt-PA auch noch jenseits dieses Dosisgrenzwertes sicher ist (Shaltoni et al. 2007).

Auch ein kombinierter Einsatz verschiedener mechanischer Rekanalisationsinstrumente, wie beispielsweise der Stentriever und des Penumbra Systems ist möglich. Die simultane Anwendung von Thrombusextraktion und -aspiration reduziert das Risiko distaler Embolisation. Auch die beiden FDA-zugelassenen Instrumente Penumbra System und MERCI Retriever können sich ergänzend eingesetzt werden (Hui et al. 2011). 


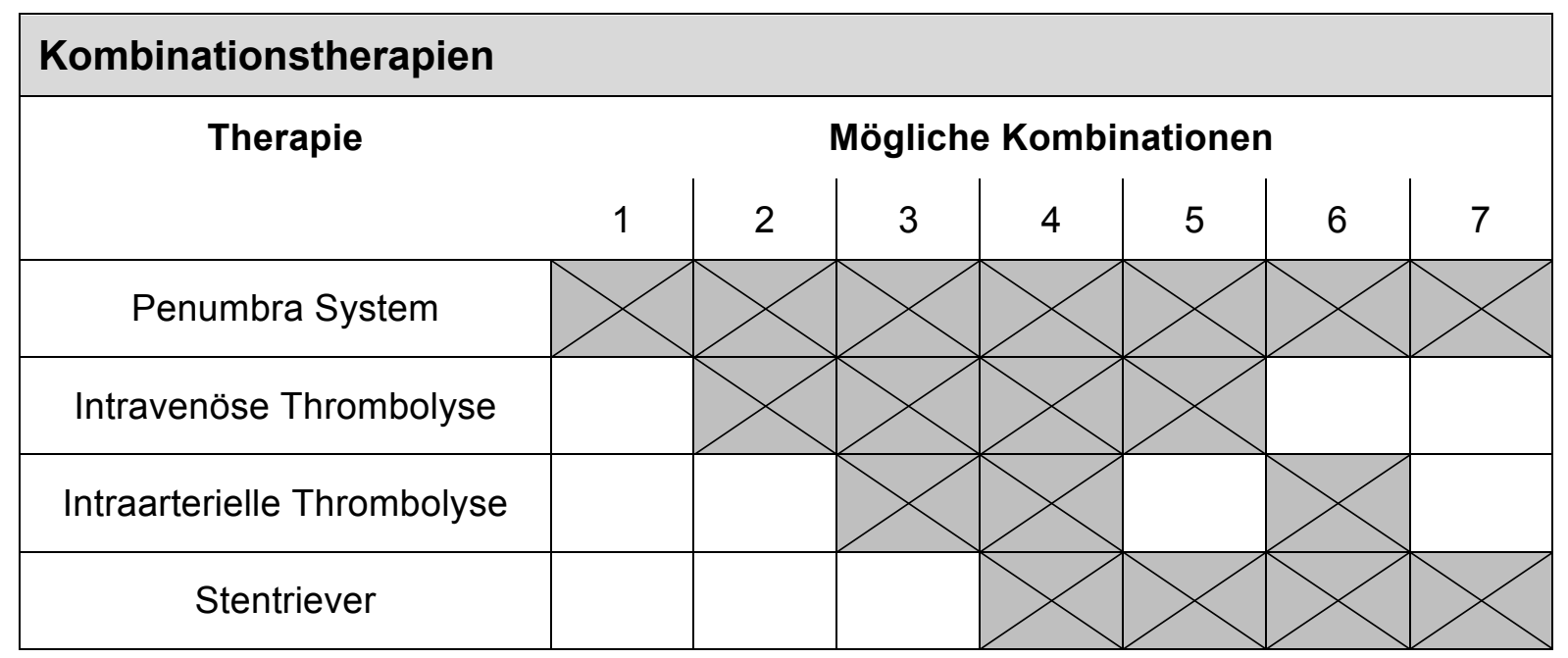

Tabelle 3 - Übersicht über die möglichen Therapieformen in Kombination mit dem Penumbra System. Modifiziert nach Kreusch und Knauth 2011, Seite 92.

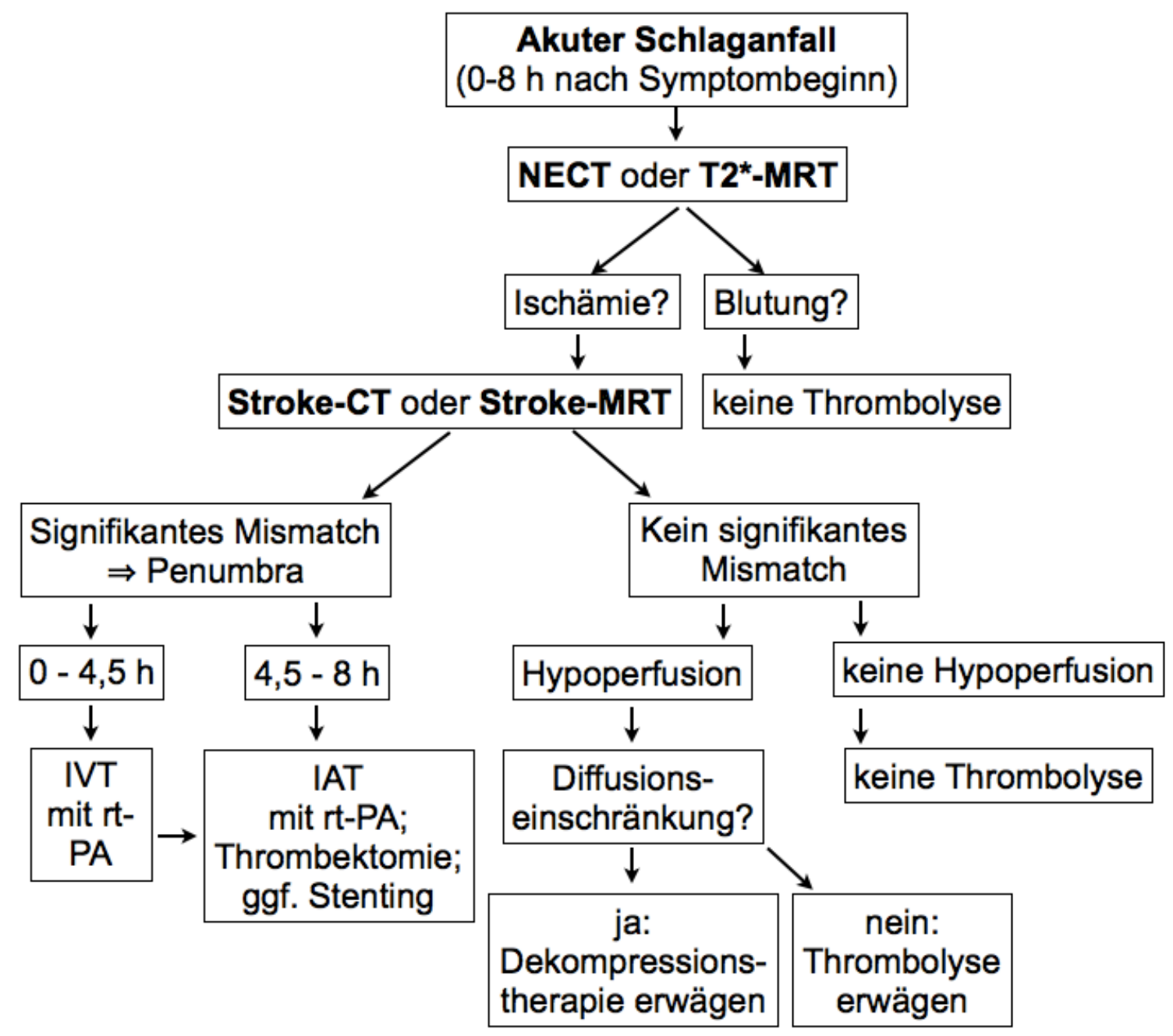

Abbildung 11 - Flussdiagramm einer möglichen Diagnostik und Akutversorgung eines ischämischen Schlaganfalls im vorderen Gefäßkreislauf. Modifiziert nach Harjung 2009, Seite 17. 


\begin{tabular}{|c|c|c|c|c|c|c|c|c|c|c|c|c|c|c|}
\hline & 总的 & $\begin{array}{l}\stackrel{0}{0} \\
\dot{0} \\
0\end{array}$ & $\begin{array}{l}\text { ㅇ } \\
\text { 음 }\end{array}$ & 这 & $\begin{array}{l}\circ \\
\infty \\
\infty \\
\infty\end{array}$ & $\begin{array}{l}\stackrel{\circ}{\circ} \\
\stackrel{+}{N}\end{array}$ & $\begin{array}{l}\text { वீ } \\
\dot{\sigma} \\
\stackrel{5}{5}\end{array}$ & $\begin{array}{l}\text { ㅇ } \\
\text { 음 }\end{array}$ & $\begin{array}{l}\circ \\
\text { å }\end{array}$ & $\begin{array}{l}\circ \\
m \\
m \\
0\end{array}$ & $\begin{array}{l}\circ 0 \\
\text { oे } \\
\sigma\end{array}$ & 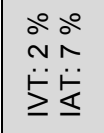 & 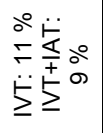 & $\begin{array}{l}\circ \\
m \\
0 \\
0\end{array}$ \\
\hline & 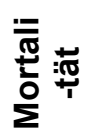 & $\stackrel{\circ}{\stackrel{\circ}{\circ}}$ & $\begin{array}{l}\text { ஓீ } \\
\mp\end{array}$ & $\begin{array}{l}\stackrel{0}{ } \\
\dot{\sim} \\
\stackrel{N}{N}\end{array}$ & $\begin{array}{l}\stackrel{0}{0} \\
10 \\
0 \\
0\end{array}$ & $\begin{array}{l}\stackrel{\circ}{\circ} \\
\text { ก }\end{array}$ & $\begin{array}{l}\circ \\
0 \\
0 \\
\stackrel{N}{N}\end{array}$ & $\begin{array}{l}\text { ¿̊ } \\
\stackrel{2}{\sim}\end{array}$ & $\begin{array}{l}\text { ¿̊ } \\
\text { in } \\
\omega^{\circ}\end{array}$ & $\begin{array}{l}\stackrel{0}{0} \\
\wp\end{array}$ & $\begin{array}{l}\stackrel{0}{0} \\
\wp\end{array}$ & 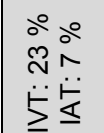 & 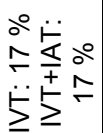 & $\begin{array}{l}\text { ¿ீ } \\
\text { in }\end{array}$ \\
\hline & 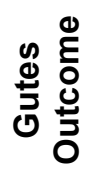 & $\begin{array}{l}\text { o } \\
m \\
0 \\
0 \\
0\end{array}$ & 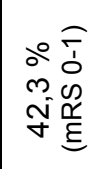 & 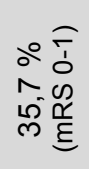 & 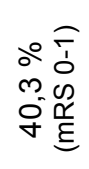 & 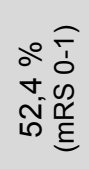 & 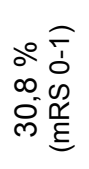 & $\begin{array}{l}\text { ஃ̊ } \\
\text { o }\end{array}$ & $\frac{\partial}{\frac{\sigma}{\sigma}}$ & $\begin{array}{l}\stackrel{0}{9} \\
\text { m }\end{array}$ & $\begin{array}{l}\circ \\
0 \\
\dot{q}\end{array}$ & 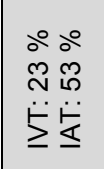 & 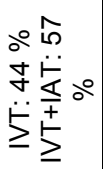 & 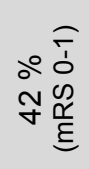 \\
\hline & 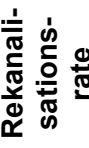 & $\begin{array}{l}\dot{\leftarrow} \\
\dot{x}\end{array}$ & $\begin{array}{l}\dot{\leftarrow} \\
\dot{x}\end{array}$ & $\begin{array}{l}\dot{\leftarrow} \\
\dot{x}\end{array}$ & $\begin{array}{l}\dot{\leftarrow} \\
\dot{x}\end{array}$ & $\begin{array}{l}\dot{\leftarrow} \\
\dot{x}\end{array}$ & $\frac{\circ}{\hat{n}}$ & $\begin{array}{l}\stackrel{0}{ } \\
\varnothing\end{array}$ & $\frac{\circ}{n^{2}}$ & $\begin{array}{l}\text { ¿0 } \\
\text { i⿱ }\end{array}$ & $\begin{array}{l}\text { ○ } \\
\infty \\
i n\end{array}$ & 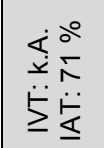 & 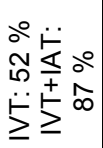 & 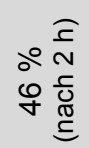 \\
\hline $\begin{array}{c}\overline{\overline{0}} \\
\overline{1}\end{array}$ & 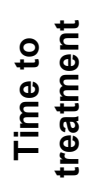 & 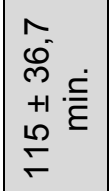 & $\begin{array}{l}\stackrel{\dot{\bar{\varepsilon}}}{\bar{\varepsilon}} \\
\stackrel{0}{\sim}\end{array}$ & $\begin{array}{l}\stackrel{\dot{E}}{\varepsilon} \\
\dot{0} \\
\stackrel{N}{N}\end{array}$ & $\begin{array}{l}\dot{<} \\
\dot{x}\end{array}$ & $\stackrel{\mathscr{N}}{N}$ & $\underset{\sim}{\stackrel{\sim}{\sim}}$ & ָ̃ & 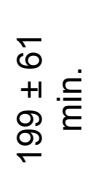 & 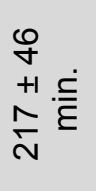 & $\begin{array}{l}\bar{m} \\
+1 \\
+\dot{\underline{E}} \\
\dot{g}\end{array}$ & 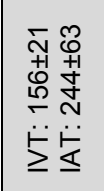 & 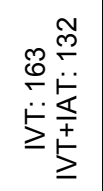 & 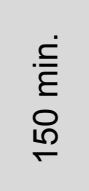 \\
\hline 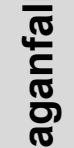 & 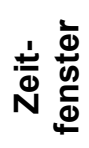 & ç & $\begin{array}{l}\text { ᄃ } \\
\text { Lp } \\
\text { m. }\end{array}$ & \begin{tabular}{l}
\multicolumn{1}{c}{} \\
0 \\
0
\end{tabular} & 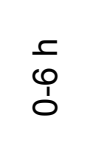 & 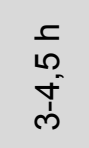 & \begin{tabular}{l}
\multicolumn{1}{c}{} \\
0 \\
0
\end{tabular} & \begin{tabular}{l}
\multicolumn{1}{c}{} \\
0 \\
0
\end{tabular} & \begin{tabular}{l}
\multicolumn{1}{c}{} \\
0 \\
0
\end{tabular} & $\begin{array}{l}\frac{c}{m} \\
\text { o }\end{array}$ & c) & 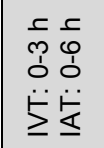 & 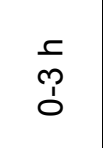 & ç \\
\hline 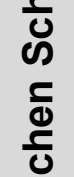 & 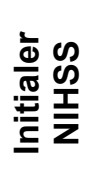 & $\underset{\square}{\nabla}$ & $\mp$ & $\stackrel{\nabla}{\sim}$ & $\mp$ & $\sigma$ & $\stackrel{\Sigma}{\Sigma}$ & 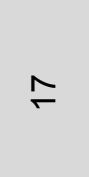 & $\stackrel{\nabla}{\sim}$ & $\stackrel{\infty}{\sim}$ & $\stackrel{\circ}{-}$ & 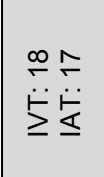 & 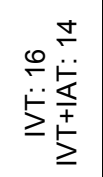 & $\stackrel{\varphi}{\sim}$ \\
\hline 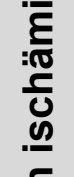 & 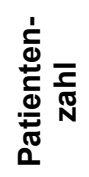 & $\frac{N}{m}$ & $\underset{\sim}{\stackrel{N}{N}}$ & $\frac{m}{m}$ & \& & $\stackrel{\infty}{\frac{\nabla}{\sigma}}$ & $\stackrel{\leftrightarrow}{\sim}$ & $\stackrel{\bar{N}}{\sim}$ & $\stackrel{\square}{\check{r}}$ & ০ & 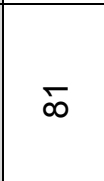 & 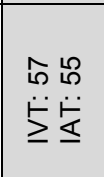 & 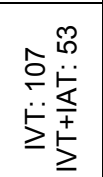 & రె \\
\hline $\begin{array}{l}\frac{0}{2} \\
\frac{1}{0} \\
0 \\
0 \\
0 \\
0\end{array}$ & 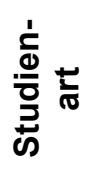 & 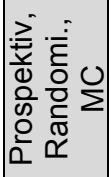 & 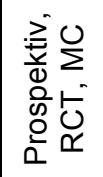 & 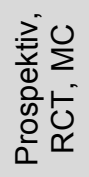 & 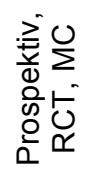 & 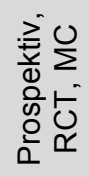 & 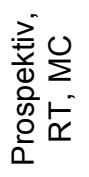 & 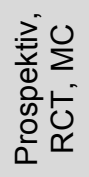 & 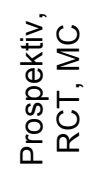 & 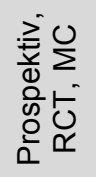 & 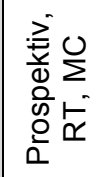 & 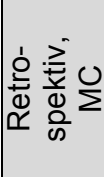 & 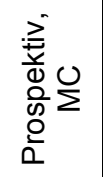 & 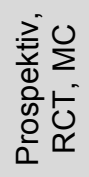 \\
\hline 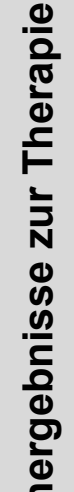 & $\begin{array}{l}\frac{0}{0} \\
\stackrel{0}{0} \\
\text { ஸे }\end{array}$ & 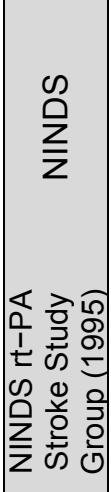 & 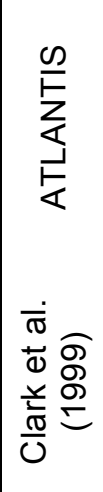 & 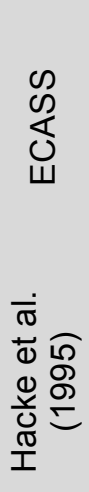 & 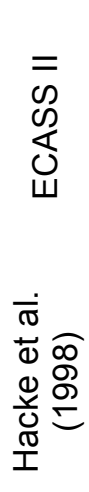 & 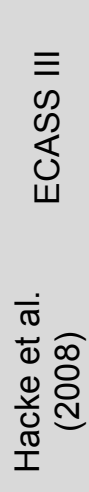 & 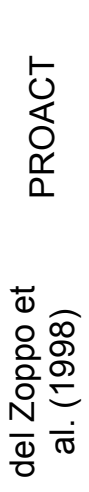 & 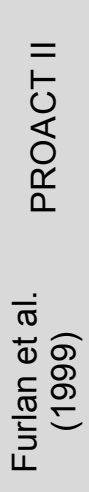 & 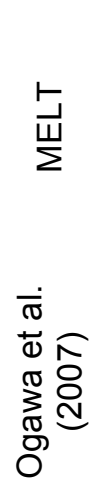 & 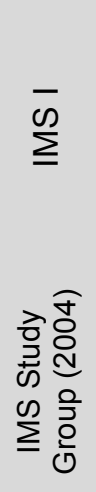 & 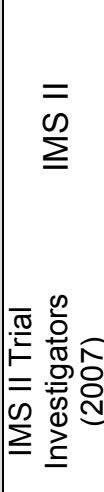 & 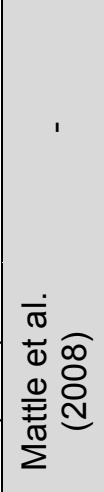 & 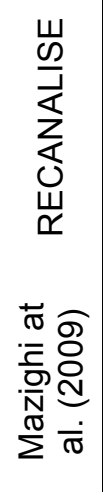 & 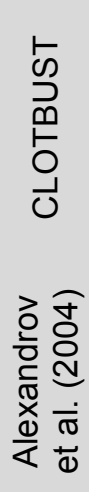 \\
\hline 竞 & & & & IVT & & & & IAT & & IVT & + IAT & $\begin{array}{l}\text { IVT } \\
\text { vs. } \\
\text { IAT }\end{array}$ & $\begin{array}{c}\text { IVT } \\
+ \\
\text { MT }\end{array}$ & $\begin{array}{c}\text { IVT } \\
+ \\
\text { ST }\end{array}$ \\
\hline
\end{tabular}




\begin{tabular}{|c|c|c|c|c|c|c|c|c|c|c|c|c|c|}
\hline \multirow{11}{*}{ 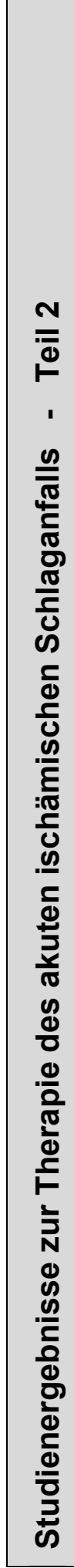 } & है을 & $\begin{array}{l}\stackrel{0}{\circ} \\
\stackrel{0}{\circ}\end{array}$ & ஃ̊ & $\begin{array}{l}\stackrel{\circ}{N} \\
\stackrel{5}{\leftarrow}\end{array}$ & $\stackrel{\circ}{\circ}$ & $\stackrel{\circ}{\wedge}$ & $\stackrel{\circ}{\circ}$ & $\begin{array}{l}\text { o } \\
\text { v } \\
\text { v }\end{array}$ & $\stackrel{\circ}{\circ}$ & $\begin{array}{l}\circ \\
\infty \\
\infty\end{array}$ & $\begin{array}{l}\stackrel{0}{0} \\
\infty\end{array}$ & $\stackrel{\circ}{\circ}$ & $\begin{array}{l}\stackrel{0}{\circ} \\
\stackrel{0}{\circ}\end{array}$ \\
\hline & 竘 & 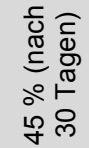 & $\begin{array}{l}\stackrel{0}{ } \\
\text { O }\end{array}$ & $\begin{array}{l}\text { o } \\
\infty \\
\text { ले }\end{array}$ & $\begin{array}{l}\stackrel{\circ}{\circ} \\
\stackrel{L}{N}\end{array}$ & 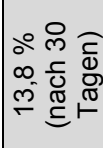 & 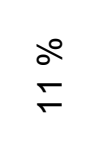 & $\begin{array}{l}{ }^{\circ} \\
\omega^{\circ} \\
\infty\end{array}$ & 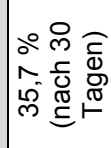 & $\begin{array}{l}\stackrel{0}{0} \\
\stackrel{0}{y} \\
\stackrel{y}{y}\end{array}$ & $\begin{array}{l}\stackrel{\circ}{\circ} \\
\text { 尺े }\end{array}$ & $\begin{array}{l}\circ \\
0 \\
0\end{array}$ & $\begin{array}{l}\stackrel{0}{0} \\
\text { मे }\end{array}$ \\
\hline & 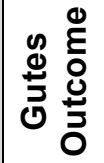 & 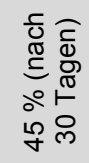 & $\begin{array}{l}\stackrel{0}{0} \\
\text { q }\end{array}$ & $\begin{array}{l}\stackrel{\circ}{\circ} \\
\stackrel{2}{N}\end{array}$ & $\begin{array}{l}\stackrel{\circ}{\Im} \\
\stackrel{\sim}{*}\end{array}$ & $\begin{array}{l}\text { o̊ } \\
\text { o } \\
\text { No }\end{array}$ & $\begin{array}{l}\stackrel{0}{\circ} \\
\infty \\
+\end{array}$ & $\frac{\stackrel{0}{0}}{\stackrel{\infty}{\infty}}$ & 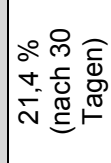 & $\frac{\stackrel{0}{0}}{\hat{N}}$ & $\begin{array}{l}\stackrel{\circ}{\circ} \\
\stackrel{2}{N}\end{array}$ & $\begin{array}{l}\stackrel{\circ}{N} \\
\stackrel{\Delta}{ }\end{array}$ & $\begin{array}{l}\stackrel{0}{0} \\
\dot{m}\end{array}$ \\
\hline & 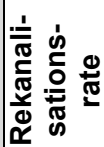 & $\begin{array}{l}\circ \\
\circ \\
\circ\end{array}$ & $\begin{array}{l}\circ \\
\circ \\
\circ\end{array}$ & $\begin{array}{l}\circ \\
0 \\
0 \\
\infty\end{array}$ & $\begin{array}{l}\stackrel{0}{0} \\
\stackrel{\infty}{ }\end{array}$ & 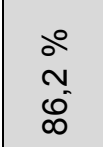 & $\begin{array}{l}\stackrel{0}{0} \\
\text { §̊ }\end{array}$ & $\begin{array}{l}\circ 0 \\
\hat{0}\end{array}$ & $\begin{array}{l}\circ \\
\text { ¿ }\end{array}$ & $\begin{array}{l}\circ \\
\stackrel{0}{+} \\
\infty\end{array}$ & $\begin{array}{l}\text { ○̊ } \\
\text { in }\end{array}$ & $\begin{array}{l}\stackrel{0}{0} \\
0 \\
0\end{array}$ & $\begin{array}{l}\circ \\
10 \\
80 \\
0\end{array}$ \\
\hline & 苍 & $\underset{x}{\dot{x}}$ & $\begin{array}{l}\stackrel{\dot{\bar{E}}}{E} \\
\infty \\
\text { ले }\end{array}$ & 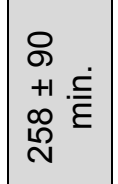 & $\frac{\dot{E}}{\frac{E}{5}}$ & 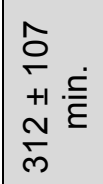 & 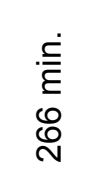 & 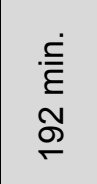 & $\frac{\dot{\bar{E}}}{\frac{10}{m}}$ & 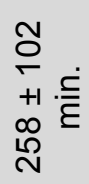 & $\begin{array}{l}\dot{\bar{E}} \\
\dot{m} \\
0\end{array}$ & $\begin{array}{l}\stackrel{\dot{S}}{E} \\
\stackrel{n}{N}\end{array}$ & $\begin{array}{l}\dot{\bar{E}} \\
\infty \\
\stackrel{D}{N}\end{array}$ \\
\hline & 意 & $\begin{array}{c}c \\
\infty \\
\vdots \\
d\end{array}$ & $\begin{array}{l}\frac{c}{m} \\
\stackrel{N}{\dot{N}}\end{array}$ & 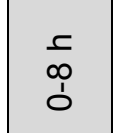 & $\frac{c}{0}$ & 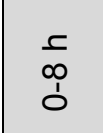 & 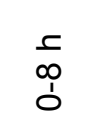 & $\frac{c}{\frac{1}{\sigma}}$ & 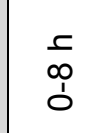 & \begin{tabular}{l}
\multicolumn{1}{c}{} \\
0 \\
0 \\
0
\end{tabular} & $\begin{array}{l}c \\
\infty \\
0 \\
0\end{array}$ & $\begin{array}{l}c \\
\text { o } \\
0 \\
0\end{array}$ & 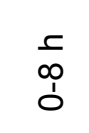 \\
\hline & 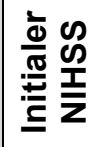 & $\bar{\sim}$ & $\begin{array}{l}\infty \\
\mathcal{N}^{-}\end{array}$ & $\stackrel{0}{\stackrel{0}{\sim}}$ & $\stackrel{20}{\sim}$ & 尺 & $\stackrel{Ð}{\leftarrow}$ & $\stackrel{\infty}{-}$ & 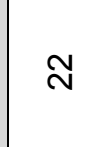 & 尺 & $\bar{\sim}$ & $\stackrel{\infty}{\leftarrow}$ & $\stackrel{\text { の }}{\square}$ \\
\hline & 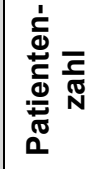 & $\stackrel{\mathscr{N}}{ }$ & 10 & $\stackrel{\stackrel{\sim}{\sim}}{\sim}$ & $\stackrel{20}{\leftarrow}$ & $\stackrel{\text { N }}{ }$ & $\hat{\sim}$ & $\grave{\sim}$ & $\stackrel{\infty}{\sim}$ & $\underset{\square}{\check{\Xi}}$ & $\stackrel{\Xi}{\sim}$ & $\stackrel{\llcorner}{N}$ & $\underset{\leftarrow}{\oplus}$ \\
\hline & 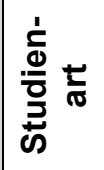 & 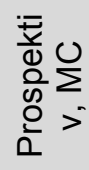 & 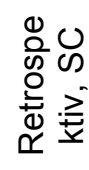 & 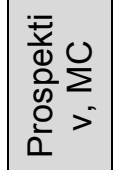 & 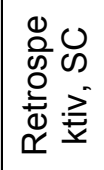 & 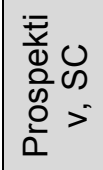 & 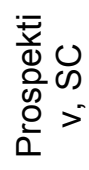 & 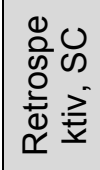 & 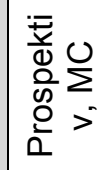 & 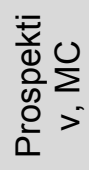 & 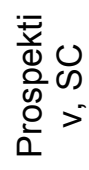 & 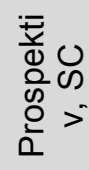 & 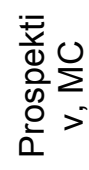 \\
\hline & 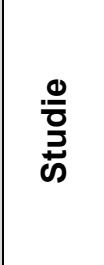 & 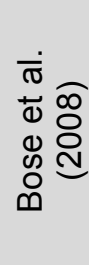 & 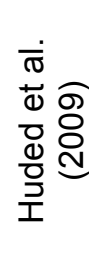 & 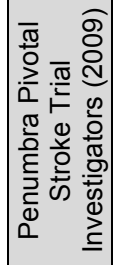 & 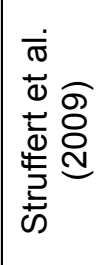 & 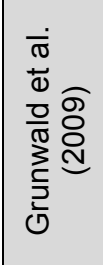 & 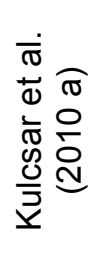 & 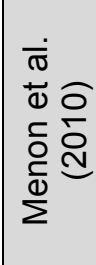 & 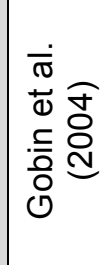 & 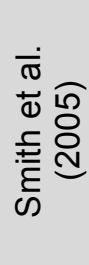 & 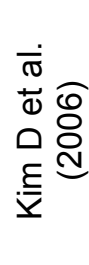 & 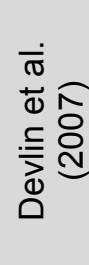 & 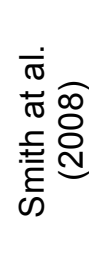 \\
\hline & & & & numbra & a Syst & em & & & & MEF & Cl Ret & iever & \\
\hline
\end{tabular}

Tabelle 4 - Übersicht über die wichtigsten Veröffentlichungen zur Therapie des akuten ischämischen Schlaganfalls mit Schwerpunkt auf der mechanischen Rekanalisation.

IVT: intravenöse Thrombolyse; IAT: intraarterielle Thrombolyse; k.A.: keine Angabe; MT: mechanische Thrombektomie; ST: Sonothrombolyse, SC: Single Center; MC: Multi Center Modifiziert nach Kreusch und Knauth 2011, Seite 87-88. 


\subsubsection{Medikamentöse Sekundärprophylaxe}

Bereits in der Frühphase eines ischämischen Schlaganfalls sollte die Prophylaxe eines Schlaganfalls bzw. Schlaganfallrezidivs eingeleitet werden, da Zweitinfarkte eine wesentlich schlechtere Prognose als der Primärinfarkt haben.

Entsprechend den deutschen Leitlinien wird in der Frühphase die Verabreichung von Acetylsalicylsäure $(100-300 \mathrm{mg} / \mathrm{d})$ empfohlen. Die Heparinisierung in für die partielle Thromboplastinzeit relevanter Dosierung oder die entsprechende Gabe niedermolekularer Heparine ist in unausgewählten Patientenkollektiven nicht wirksam (AWMF-Leitlinien 2008).

Die optimale Sekundärprävention des ischämischen Schlaganfalls ist noch nicht abschließend geklärt (Howard et al. 2007).

\subsubsection{Vorbeugung und Behandlung von Komplikationen}

Ein erlittener Schlaganfall prädisponiert für zahlreiche medizinische Komplikationen, wie beispielsweise Pneumonie, tiefer Beinvenenthrombosen und Lungenembolie. Bei bakteriellen Infektionen wird eine gezielte und frühzeitige antibiotische Behandlung empfohlen. Früh begonnene Physiotherapie, Stützstrümpfe und niedrigdosierte Heparine können vor solchen Komplikationen schützen.

Das raumfordernde Hirnödem und erhöhter intrakranieller Druck sind lebensbedrohliche Komplikationen, die nach einem Schlaganfall auftreten können. Ein Hirnödem tritt zumeist 2 - 5 Tage nach dem Schlaganfall auf, wobei klinische Symptome der Diagnose meist vorausgehen (Qureshi et al. 2003). Die frühe Hemikraniektomie innerhalb von 48 Stunden nach Symptombeginn wird bei Patienten > 60 Jahren und malignem Mediainfarkt nach individueller Abwägung empfohlen (AWMF-Leitlinien 2008), da durch diese Maßnahme im Vergleich zur konservativen medikamentösen Therapie die Überlebenswahrscheinlichkeit und das funktionelle Endergebnis weiter verbessert werden können (Vahedi et al. 2007).

Die Behandlung auf einer Stroke-Unit mit erfahrenem Personal reduziert die Komplikationsrate und verbessert die Prognose des Patienten, unabhängig davon ob er einer thrombolytischen Therapie unterzogen wurde.

\subsubsection{Rehabilitation}

Das Ziel der Rehabilitation ist, Patienten in die Lage zu versetzen, möglichst gute physische, intellektuelle und/oder soziale Funktionen zu erreichen und zu erhalten. 
Der umgehende Beginn der Rehabilitation ist eine der wesentlichen Komponenten der Behandlung auf einer Stroke-Unit und reduziert die Inzidenz von Komplikationen, welche meist mit der Immobilisation verbunden sind (Stroke Unit Trialists' Collaboration 2007).

Ein multidisziplinäres Rehabilitationsteam besteht üblicherweise aus Ärzten, Pflegepersonal, Physiotherapeuten, Ergotherapeuten und Logopäden. Im Anschluss an einen Schlaganfall wird eine Rehabilitation empfohlen, jedoch sind die besten Methoden weiterhin unklar (Ringleb et al. 2008).

\subsection{Aktueller Stand der Forschung}

Die These, auf welche sich die heutigen Therapien des ischämischen Schlaganfalls stützen, basiert auf der impliziten Annahme, dass die schnelle, vollständige Reperfusion minderdurchbluteten Hirngewebes zu besseren langfristigen klinischen Behandlungsergebnissen führt (siehe Abbildung 13, Seite 43) (Rha und Saver 2007).

In der Literatur werden drei Typen von Reperfusionsstrategien beschrieben (siehe Abbildung 12):

1. Rekanalisation oder antegrade Reperfusion,

2. Globale Reperfusion (Flusssteigerung oder transarterielle retrograde Reperfusion),

3. Transvenöse retrograde Reperfusion (Flussumkehr) (Nogueira et al. 2009).

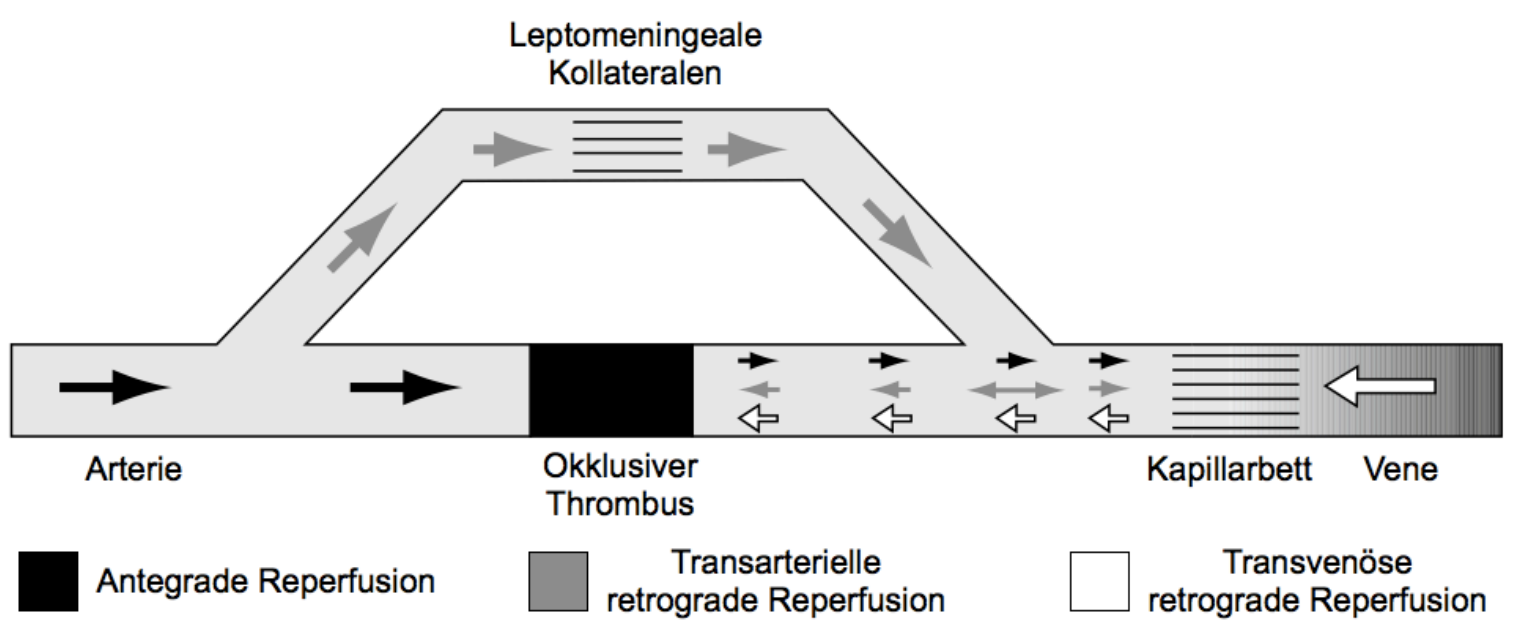

Abbildung 12 - Schematisches Diagramm des neurovaskulären Gefäßbettes und der möglichen Reperfusionsstrategien. Einige dieser Verfahren bleiben experimentell. Modifiziert nach Gonzalez et al. 2010, Seite 268. 
Im Folgenden soll dabei auf den aktuellen Forschungstand zur Rekanalisation oder antegraden Reperfusion als den am weitesten verbreiteten Mechanismus zur Flusswiederherstellung in Rahmen der Therapieoptionen eingegangen werden.

Die intravenöse rt-PA-Applikation ist heute die nützlichste, wissenschaftlich belegte Therapieform für den akuten ischämischen Schlaganfall. Sie ist weit verbreitet und steht nach dem Ausschluss einer Blutung schnell zur Verfügung. Diese Fibrinolytikagabe ist aber nur in einem Zeitfenster bis zu 3 Stunden nach Symptombeginn zugelassen und es zeigt sich ein gradueller Wirksamkeitsverlust über die Zeit (Hacke et al. 2008). Bei einem späteren intravenösen Therapiebeginn kommt es zu einem Anstieg des Sterberisikos und es profitieren weniger Patienten von einer IVT (Lees et al. 2010).

Weitere Einschränkungen dieser Standardtherapie sind die geringe Rekanalisationsrate bei proximalen, langstreckigen Gefäßverschlüssen und der Misserfolg bei der Gefäßwiedereröffnung abhängig von der Thrombuslänge (Alexandrov et al. 2000). Während bei Thrombuslängen unter $4 \mathrm{~mm}$ durch IVT in über $70 \%$ der Fälle eine Rekanalisation erreicht werden kann, sinkt die Rekanalisationswahrscheinlichkeit auf $1 \%$, wenn die Thrombuslänge $8 \mathrm{~mm}$ überschreitet (Riedel et al. 2011).

Obwohl die IVT die aktuelle Standardtherapie des akuten ischämischen Schlaganfalls ist, steht diese Therapie nur für eine Minderheit der betroffenen Patienten zur Verfügung. Der Hauptgrund für die eingeschränkte Einsetzbarkeit sind das beschriebene enge Zeitfenster, verbunden mit der Notwendigkeit einer Bildgebung des Kopfes zum Ausschluss einer intrazerebralen Blutung vor Therapiebeginn (Barber et al. 2001). In Deutschland bekommen zur Zeit etwa $14 \%$ der Patienten mit akutem ischämischen Schlaganfall eine IVT im Zeitfenster von 3 Stunden nach Symptombeginn (Grau et al. 2010).

In den großen randomisierten, placebo-kontrollierten und doppelblinden Studien zur IVT (ECASS, NINDS, ATLANTIS) zeigte sich, dass im Vergleich zur Placebo-Gabe nur maximal jeder 8. Patient (13\%) zusätzlich von dieser Therapieform profitiert und danach ein selbstständiges Leben (weitgehend) ohne Behinderungen im Alltag (mRS $\leq 2$ ) führen kann (NINDS t-PA Stroke Study Group 1997) (siehe Tabelle 4, Seite 36/37). 
Daraus ersichtlich wird der hohe Bedarf an neuen therapeutischen Alternativen für eine sichere Reperfusion bei solchen Patienten, die nicht für IVT in Frage kommen oder nicht von ihr profitieren.

Es besteht eine wichtige Korrelation zwischen Rekanalisation und klinischem Behandlungsergebnis, wobei die Wiedereröffnung des Gefäßes sehr stark mit einem verbesserten neurologisch-funktionellem Ergebnis assoziiert ist (Rha und Saver 2007). Neben dem Zeitfaktor ist der Rekanalisationsstatus der stärkste Prädiktor für das klinische Endergebnis von Schlaganfallpatienten (Khatri et al. 2009; Nogueira et al. 2009).

Die Bedeutung der arteriellen Rekanalisation nach einem Schlaganfall konnte in einer Metaanalyse von 53 Studien mit 2066 Patienten deutlich gezeigt werden: Eine erfolgreiche Rekanalisation innerhalb von 6 Stunden nach Symptombeginn war dabei mit einem vier- bis fünffachem Anstieg der Odds Ratio von gutem funktionellem Outcome und einer vier- bis fünffachen Reduktion der Odds Ratio von Mortalität assoziiert (Rha und Saver 2007). Beim Vergleich der verschiedenen Rekanalisationsverfahren ergaben sich aus der Metastudie folgende Rekanalisationsraten:

- Spontanrekanalisation (in 24 Stunden): $24.1 \%$

- Intravenöse Fibrinolyse: $46.2 \%$

- Intraarterielle Fibrinolyse: $63.2 \%$

- Kombinierte intravenöse und intraarterielle Thrombolyse: $67.5 \%$

- Mechanische Thrombolyse: $83.6 \%$.

Der Nachweis, dass die Rekanalisation und Reperfusion des distalen Gefäßbettes in entsprechend ausgewählten Patienten entscheidend ist, um ein gutes funktionelles Endergebnis zu erzielen, hat großes Interesse und die weitere Erforschung von endovaskulären Behandlungsmöglichkeiten mit hohen Rekanalisationserfolgsraten des akuten Schlaganfalls ausgelöst. Analog zur Therapie der myokardialen Ischämie rücken mechanische Techniken in der Akutphase in den Forschungsfokus und es findet ein Wechsel von der medikamentösen zur endovaskulären Therapie statt.

Seit Einführung der lokalen intraarteriellen Thrombolyse durch Hermann Zeumer im Jahr 1982 haben sich im Bereich der endovaskulären Schlaganfalltherapie die Technik und die zur Verfügung stehenden Instrumente sehr weiterentwickelt (Zeumer et al. 1982). Neuartige mechanische Rekanalisationsinstrumente ermöglichen eine 
schnelle und hohe Rekanalisationsrate bei geringem Auftreten von Komplikationen. Die endovaskuläre Behandlung stellt eine vielversprechende Alternative mit theoretischen Vorteilen gegenüber der intravenösen Therapie dar, wie beispielsweise höhere Rekanalisationsraten und längere therapeutische Zeitfenster. Die aktuell verfügbaren Instrumente erzielen technische Erfolgsraten von bis zu 70-100\% bei gleichzeitig geringem Auftreten von peri- oder postprozeduralen Komplikationen. Symptomatische intrakranielle Blutungen sind mit $<10 \%$ die häufigste Komplikation, periprozedurale Todesfälle sind sehr selten (<1\%) (Serna-Candel et al. 2010).

Neue Studien deuten darauf hin, dass letztlich die kombinierte intravenöse und endovaskuläre Therapie die bevorzugte Strategie für die zerebrale Rekanalisation sein wird (IMS Study Group 2004; Mazighi et al. 2009). Sie verbindet die Vorteile eines schnellen Therapiestarts durch IVT und hoher Rekanalisationsraten durch endovaskuläre Optionen.

Dass höhere Gefäßrekanalisationsraten im Zeitfenster jenseits von 4,5 Stunden nach Symptombeginn nicht unbedingt $z u$ einem besseren klinischen Rehabilitationsergebnis nach 3 Monaten führen, ergibt sich aus der Analyse der verfügbaren Studienergebnisse (siehe Abbildung 34, Seite 92) (Alexandrov 2010). Selbst von einer frühzeitigen Thrombektomie werden Patienten möglicherweise nicht profitieren, sofern das Gefäß-abhängige Hirngewebe schon ausgedehnt infarziert ist.

In den Forschungsfokus rückt daher zunehmend die exakte Patientenauswahl für die Vielzahl der zur Verfügung stehenden Schlaganfalltherapien. Bisher fehlen einheitliche Standards, um eine normierte Auswahl von Patienten, welche wahrscheinlich von einer endovaskulären Therapie profitieren, zu ermöglichen. 


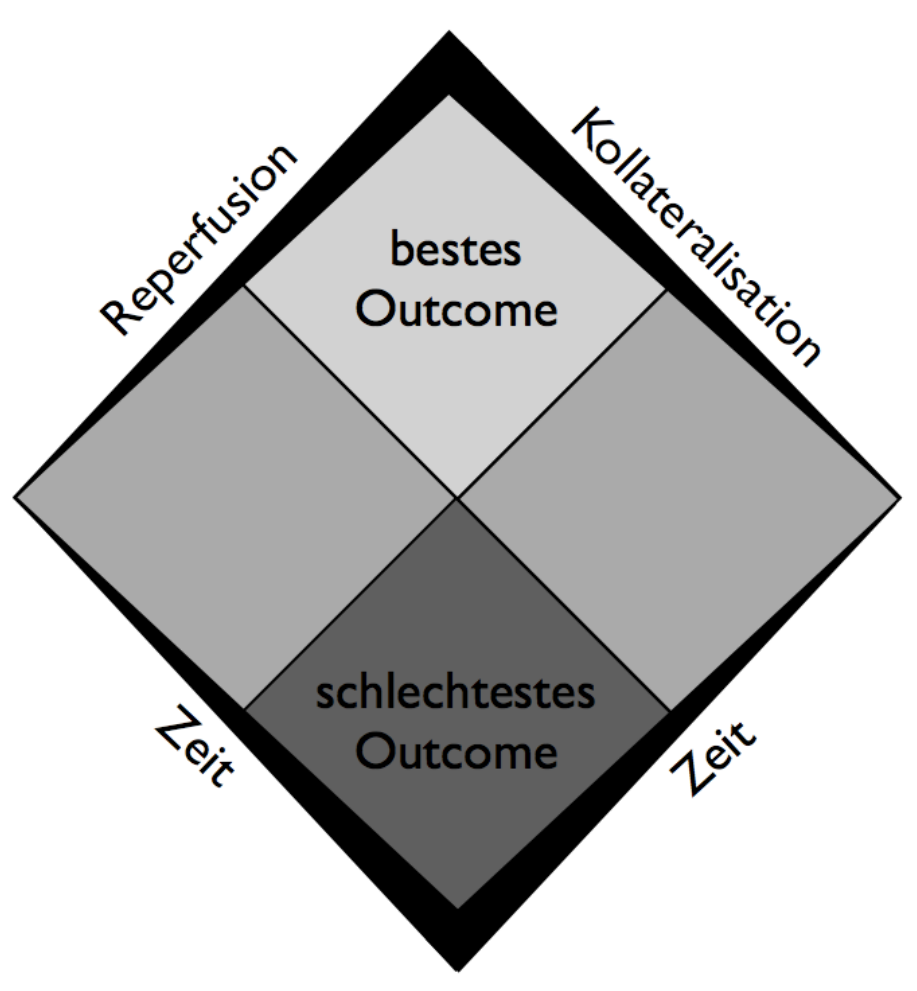

Abbildung 13 - Zusammenhang zwischen Reperfusion, Gefäßkollateralisation, der Zeit und dem klinischen Endergebnis bei akutem ischämischen Schlaganfall. Die Hypothese, dass die schnelle Reperfusion und adäquate Kollateralisation zu einer kleineren Infarktgröße und besseren klinischen Endergebnissen führt, wohingegen eine erfolglose, sehr späte Rekanalisation bei schlechter Kollateralisation mit einer größeren Infarktgröße und einem schlechten klinischen Outcome verbunden ist, muss noch abschließend validiert werden.

\subsection{Zielsetzung und Fragestellung}

Aus Kapitel 1.4 wird ersichtlich, dass die bisherige Erfahrung zu neuen Instrumenten für die mechanische Rekanalisation von Schlaganfallpatienten vorwiegend auf retrospektiven Studien mit kleiner Fallzahl beruht.

Diese retrospektive, unkontrollierte und nicht-randomisierte Single-Center-Studie setzt die mechanische Schlaganfallbehandlung mittels Penumbra System in der Abteilung Neuroradiologie der Universitätsmedizin Göttingen in einen Kontext mit anderen Zentren und zieht ein Resultat aus dem Einsatz im klinischen Alltag.

Diese Arbeit behandelt dabei folgende Schwerpunkte:

1. Wie sind die neuroradiologischen Erfahrungen hinsichtlich der technischen Erfolgsrate bei mechanischer Rekanalisation bei Patienten mit akutem ischämischen Schlaganfall mit dem Penumbra System? Traten schwerwiegende Komplikationen auf? 
2. Profitieren die betroffenen Patienten von der mechanischen Rekanalisation? Welche Patientengruppe hat den größten Nutzen einer erfolgreichen Gefäßwiederherstellung?

3. Wie ist es im Verlauf um den klinisch-neurologischen Status der Patienten bestellt?

4. Welchen Zusammenhang zwischen erfolgreicher Rekanalisation und gutem neurologischen Behandlungsergebnis nach Rehabilitation gibt es? 


\section{Patienten und Methoden}

\subsection{Patientenkollektiv und Nachuntersuchungen}

In dieser Studie wurden insgesamt 95 Patienten erfasst, von denen 91 Patienten im Zeitraum 01. Oktober 2007 bis 31. Dezember 2010 in der Abteilung für Neuroradiologie der Universitätsmedizin Göttingen aufgrund eines zerebralen ischämischen Schlaganfalls für eine mechanische Rekanalisation ausgewählt wurden. Insgesamt 4 Patienten wurden von der aktuellen Analyse ausgeschlossen, da sich schon bei der ersten DSA-Aufnahme eine Rekanalisation des ehemals verschlossenen Gefäßes darstellte $(\mathrm{TICl}>1)$ oder das Penumbra System schließlich nicht zum Einsatz kam. Alle für die mechanische Rekanalisation ausgewählten Gefäßverschlüsse waren klinisch symptomatisch.

Bei den Patienten erfolgte eine klinisch-neurologische Aufnahmeuntersuchung, außerdem wurde eine Bildgebung des Kopfes vor der Entscheidung zur Intervention durchgeführt, CCT und / oder MRT. Die Indikationsstellung erfolgte stets im Konsens mit den behandelnden Kollegen der Abteilung für Neurologie.

Postinterventionell erfolgte die Weiterbetreuung der Patienten durch die Kollegen der neurologischen Klinik und anschließend in den meisten Fällen in einer Rehabilitationseinrichtung. Die Entwicklung des neurologischen Status wurde im Verlauf beobachtet und dokumentiert. Im Studienverlauf konnten für 11 Patienten keine Daten nach Klinik-Entlassung erhoben werden.

Für die Auswertung wurden Patientendaten aus den neuroradiologischen Interventionsprotokollen, den Dokumentationen der Abteilung für Neurologie sowie den Entlassungsberichten der weiterbehandelnden Rehabilitationseinrichtungen verwendet.

Die digital archivierten Bildmaterialien wurden in der Abteilung für Neuroradiologie an einer PACS-Workstation ausgewertet.

\subsection{Diagnostik}

Im Folgenden werden die Grundlagen und Anwendungsgebiete der zur Anwendung gekommenen neuroradiologischen Bildgebungsverfahren erläutert. 


\subsubsection{Grundprinzipien der Computertomographie}

Mittels eines Computertomographen (CT) erfolgt die ringförmige bzw. spiralförmige Abtastung des Zielobjektes durch von einer Röntgenröhre erzeugte Röntgenstrahlen, die von einem gegenüber befindlichen Detektor registriert und über die Gewinnung einer Matrix als Schichtbild ausgegeben werden können. Da Röntgenstrahlen von unterschiedlichen Gewebearten verschieden stark absorbiert werden, zeigt der akquirierte Volumendatensatz verschiedene Röntgenabsorptionswerte entsprechend den Gewebedichten, welche in Hounsfield-Einheiten (HE) angegeben werden. Die Skala reicht von -1000 bis $+3000 \mathrm{HE}$, wobei für Wasser ein Wert von $0 \mathrm{HE}$ definiert wurde.

Die modernen Computertomographen erlauben eine gleichzeitige Akquisition von bis zu 320 Zeilen mit Schichtdicken von minimal 0,3 mm. Aufgrund der Entwicklung der Spiraltechnik und Mehrzeilen-Detektoren ist heutzutage die Erfassung großer Volumendatensätze in sehr kurzer Zeit mittels Multislice-CTs möglich. Im Postprocessing der Volumendatensätze können dreidimensionale anatomische Strukturen dargestellt werden.

Für die Evaluation eines akuten Schlaganfalls spielen zwei weitere CTBildgebungstechniken, zusätzlich zur nativen CT-Technik, eine wichtige Rolle:

Bei der CT-Angiographie (CTA) werden durch zusätzliche intravenöse Applikation eines jodhaltigen KM-Bolus die Gefäße dargestellt. Nach Rekonstruktion mittels MIP (maximum intensity projection) oder Volume Rendering (VR) lassen sich frei rotierbare 3D-Darstellungen der hyperdens erscheinenden Gefäße ausgeben und erlauben eine Diagnostik von arteriellen und venösen Gefäßpathologien.

Eine weitere Möglichkeit mit besonderer Wichtigkeit für die Schlaganfallsdiagnostik ist die Perfusions-CT (CTP). Für die funktionelle Darstellung der zerebralen Perfusion wird ein intravenös applizierter jodhaltiger KM-Bolus bei seiner ersten Passage durch das Hirngewebe durch kontinuierliche, simultane und mehrschichtige Bildakquirierung verfolgt (bolus-tracking). Die zerebrale Perfusion kann anhand bestimmter Durchblutungsparameter (v.a. zerebraler Blutfluss (CBF), zerebrales Blutvolumen (CBV) und mittlere Transitzeit (MTT)) errechnet und durch farbig kodierte Bilder dargestellt werden (siehe Abbildung 8, Seite 19).

Alle CT-Untersuchungen erfolgten an einem 128-Zeilen-Computertomographen „Somatom Definition AS+“ der Firma Siemens Healthcare (Erlangen, Deutschland). 


\subsubsection{Grundprinzipien der Magnetresonanztomographie}

Die Magnetresonanztomographie (MRT) beruht physikalisch auf dem magnetischen Moment der Atomkerne (Kernspinresonanz), keine belastende Röntgenstrahlung oder andere ionisierende Strahlung werden genutzt. Atomkerne mit ungerader Ordnungs- oder Massenzahl, entsprechend den Neutronen und/oder Protonen, sind magnetische Dipole und verfügen über einen spontanen Eigendrehimpuls, den sogenannten Kernspin. Beim Anlegen eines homogenen Magnetfeldes richten sich die Atomkerne entlang der Feldrichtung aus und vollführen eine Kreiselbewegung um die Feldlinien des äußeren Magnetfeldes, diese wird als Präzessionsbewegung bezeichnet. Die Frequenz dieser Bewegung wird Larmor-Frequenz genannt und beträgt für Protonen $42,5 \mathrm{MHz}$ pro Tesla. Rotierende Wasserstoffkerne können in diesem Umstand zwischen zwei Orientierungen wechseln, beschrieben durch zwei Richtungsachsen (Quer- und Längsachse).

Durch Einstrahlung eines kurzen Hochfrequenzimpulses, entsprechend der LarmorFrequenz und senkrecht zur Richtung des äußeren Magnetfeldes, werden die Wasserstoffprotonen als Vektoren um $180^{\circ}$ in Richtung der Querachse ausgelenkt und in ihrer Präzessionsbewegung synchronisiert (Phasenkohärenz). Nach Beendigung des Anregungsimpulses erfolgt durch Relaxation die Rückkehr in die Ausgangslage. Diese Relaxationszeit wird gemessen und anhand zweier Zeitkonstanten angegeben:

Die T1-Längsrelaxation (Spin-Gitter-Relaxation) bezeichnet den Prozess der Wiederausrichtung des Vektors in Längsrichtung des äußeren Magnetfeldes und stellt somit die Zeit dar, nach der $63 \%$ der anfänglichen Längsmagnetisierung wieder erreicht sind.

Die T2-Querrelaxationszeit (Spin-Spin-Relaxation) charakterisiert die Dephrasierung der phasensynchronen Kreiselbewegung der Atomkerne, wodurch es zu einer Abnahme der Quermagnetisierung kommt.

Durch Veränderung der MRT-Systemparameter, z.B. der Pulswiederholzeit (TR, repetition time) oder der Echozeit (TE, echo time) kann man verschiedene Wichtungen einstellen: T1-, T2- oder Protonendichte-gewichtete Sequenzen.

Wesentliche Grundlage der Signalintensitäten verschiedener Gewebearten sind unterschiedliche Relaxationszeiten, Protonendichten und auch eventuelle Flussgeschwindigkeiten. Die Signalintensitäten werden als Grauwerte codiert und so lassen sich helle (hyperintense) und dunkle (hypointense) Gewebestrukturen darstellen. 
Die Magnetresonanzangiographie (MRA) ist ein diagnostisches Verfahren zur zeitlich aufgelösten angiografischen 3-dimensionalen Darstellung von arteriellen und venösen Gefäßen mit Methoden der MRT. Die nicht invasive Time-of-flight-Technik (TOF) benötigt kein KM und basiert auf dem Einstrom von ungesättigten Kernspins in das zu untersuchende Areal, das mit Hochfrequenzimpulsen vorgesättigt wurde. Nur Gefäße, welche frisch einströmendes Blut enthalten, werden dadurch hyperintens dargestellt.

Daneben bietet die invasive kontrastverstärkte MRA (CE-MRA, contrast-enhanced MRA) mit Gadolinium als KM die Möglichkeit, Blutgefäße, nach dem Prinzip des bolus-tracking darzustellen (siehe Abbildung $32 \mathrm{~A}$, Seite 78).

Die Grundlagen weiterer MRT-Sequenzen, die eine zentrale Rolle bei der Diagnostik und Therapieplanung zerebraler Ischämien spielen, werden im Folgenden beschrieben:

Die diffusionsgewichtete Sequenz (DWI, diffusion weighted imaging) ist eine T2ähnliche Sequenz und misst die Brown'sche Molekularbewegung von Wassermolekülen im Extrazellulärraum. Diffusionseinschränkungen, wie sie beispielsweise bei einem zytotoxischen Ödem in der Ischämiezone auftreten, resultieren in einer Signalminderung und in der DWI-Sequenz hyperintens. Aufgrund der technischen Ähnlichkeit zur T2-Sequenz kann es zu einem T2-Shine-Through-Effekt kommen. Dabei kann eine ältere Läsion in derselben Region durch einen verlängerten T2Effekt einen frischen Infarkt vortäuschen. Dies lässt sich durch die zusätzliche Berechnung eines scheinbaren Diffusionskoeffizienten (apparent diffusion coefficent, $A D C)$ differenzieren, sodass sich tatsächlich diffiusionsgestörte Areale auf der ADCMap hypointens darstellen.

Die Perfusionsbildgebung (perfusion weighted imaging, PWI) basiert auf der intravenösen Injektion von Gadolinium-basiertem KM, welches bei der Passage des Hirngewebes eine Signalminderung auf den T2*-Sequenzen verursacht. Eine Ischämie führt durch eine Verzögerung der Boluspassage im betreffenden Areal zu einer relativen Signalsteigerung. Für das gesamte Gehirn können Zeit-Dichte-Kurven beim first pass des Kontrastmittels erstellt und als Karten für jede Schicht dargestellt werden. Als Parameter für die Perfusionsberechnung dienen die mittlere KMPassagezeit (mean transit time, MTT) und die Zeit vom arteriellen KM-Einstrom bis zum Erreichen des Bolus-Gipfels (time to peak, TTP) pro Voxel. 
Alle MRT-Untersuchungen erfolgten an einem 3 Tesla-Magnetresonanztomographen „Magnetom Trio“ der Firma Siemens Healthcare (Erlangen, Deutschland).

\subsubsection{Grundprinzipien der Subtraktionsangiographie}

Für die heutige neuroradiologische Diagnostik und Therapie besitzt die digitale Subtraktionsangiographie (DSA) einen hohen Stellenwert und beruht auf dem invasiven Prinzip der kontrastmittelgestützten, selektiven Darstellung intra- und extrakranieller Gefäße mit Hilfe von Röntgenstrahlen.

Nach perkutaner, meist transfemoraler Punktion einer Arterie erfolgt die Einbringung einer Schleuse oder eines Katheters nach Seldinger-Technik. Entsprechend den Zielgefäßen und deren Lokalisation eignen sich verschieden Kathetertypen. Diese müssen intraprozedural im Falle einer Intervention kontinuierlich mit einer heparinisierten Kochsalzlösung gespült werden, um thrombembolische Komplikationen zu verhindern.

Zur Erstellung eines Angiogrammes wird über einen Katheter iodhaltiges Kontrastmittel gefäßselektiv in bzw. vor den Abgang des interessierenden Blutgefäßes appliziert und in hoher zeitlicher Auflösung (3-4 Bilder/Sekunde) Röntgenaufnahmen von der Zielregion angefertigt (Füllbild). Vor Kontrastmittelgabe wird ein Nativbild (Maskenbild) der gleichen Körperregion aufgenommen. Durch digitale Subtraktion der beiden Bilder voneinander wird ein Differenzbild erzeugt (Füllbild - Maskenbild = Differenzbild), welches lediglich die mit Kontrastmittel gefüllten Gefäße unter Subtraktion aller anderen störenden, röntgendichten Strukturen, wie beispielsweise Knochen, zeigt (siehe Abbildungen 14, 32 und 33, Seiten 50, 78 und 80). In der Regel haben solche DSA-Aufnahmeserien eine Auflösung von 1024 x 1024 Pixeln (1024er Matrix).

Nach Abschluss der DSA muss das punktierte Gefäß durch ein Verschlusssystem oder einen Druckverband wieder verschlossen werden. 


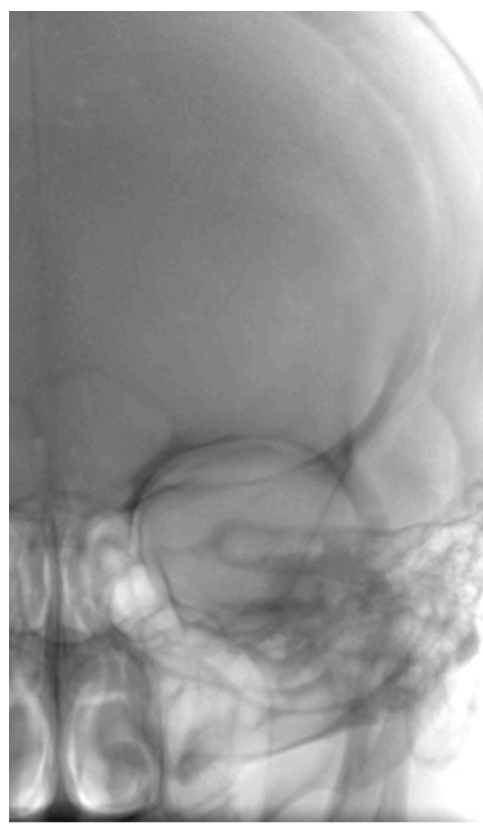

Maskenbild

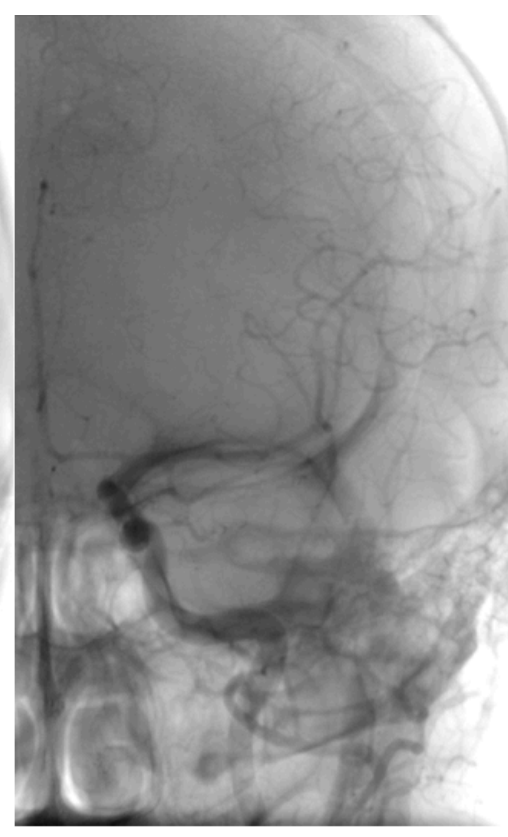

Füllbild

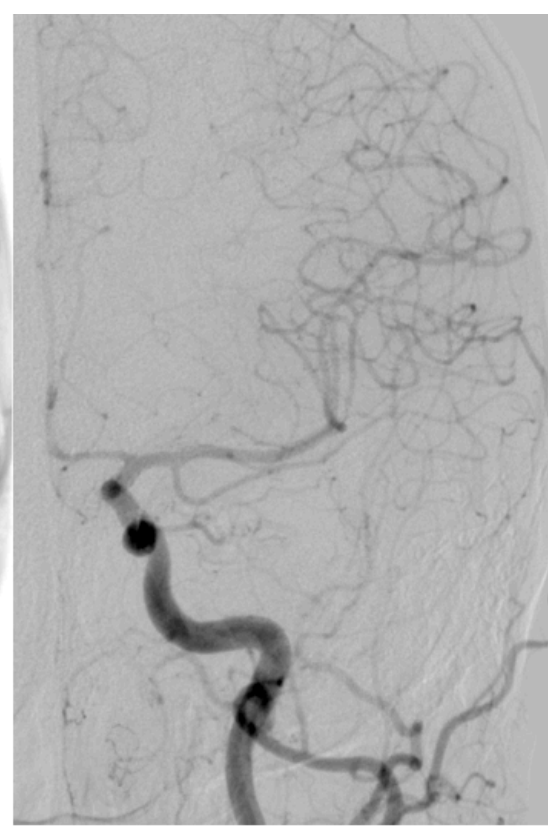

Differenzbild

$=$ (Digitale Subtraktionsangiographie)

Abbildung 14 - Prinzip der digitalen Subtraktionsangiographie. Vom Maskenbild wird das nach der Kontrastmittelinjektion erstellte Füllbild subtrahiert und lediglich die kontrastierten Gefäße sind im resultierenden Differenzbild sichtbar und beurteilbar.

Biplanare Angiographiesysteme erlauben die gleichzeitige Akquisition von zwei Projektionen/Ebenen, was die räumliche Orientierung besonders bei Interventionen optimiert. Mit einer rotationsfähigen Angiographieanlage sind ebenfalls die Akquirierung eines 3D-Volumendatensatzes (3D-Rotationsangiographie, 3D-RA) oder einer CT-ähnlichen Volumenaufnahme (Angiographische CT, ACT) möglich, aus beiden können dreidimensionale Gefäßdarstellungen erzeugt werden.

Eingesetzte Flachbild-Detektoren (flat panel detector, FPD) bieten durch eine vergrößerte Detektorfläche geometrisch distorsionsfreie Bilder und eine exzellente Auflösung im gesamten Kontrastspektrum.

Alle DSA-Untersuchungen und anschließende Interventionen erfolgten am biplanen, rotationsfähigen Angiographiesystem „AXIOM Artis dBa” mit FlachdetektorTechnologie der Firma Siemens Healthcare (Erlangen, Deutschland). 


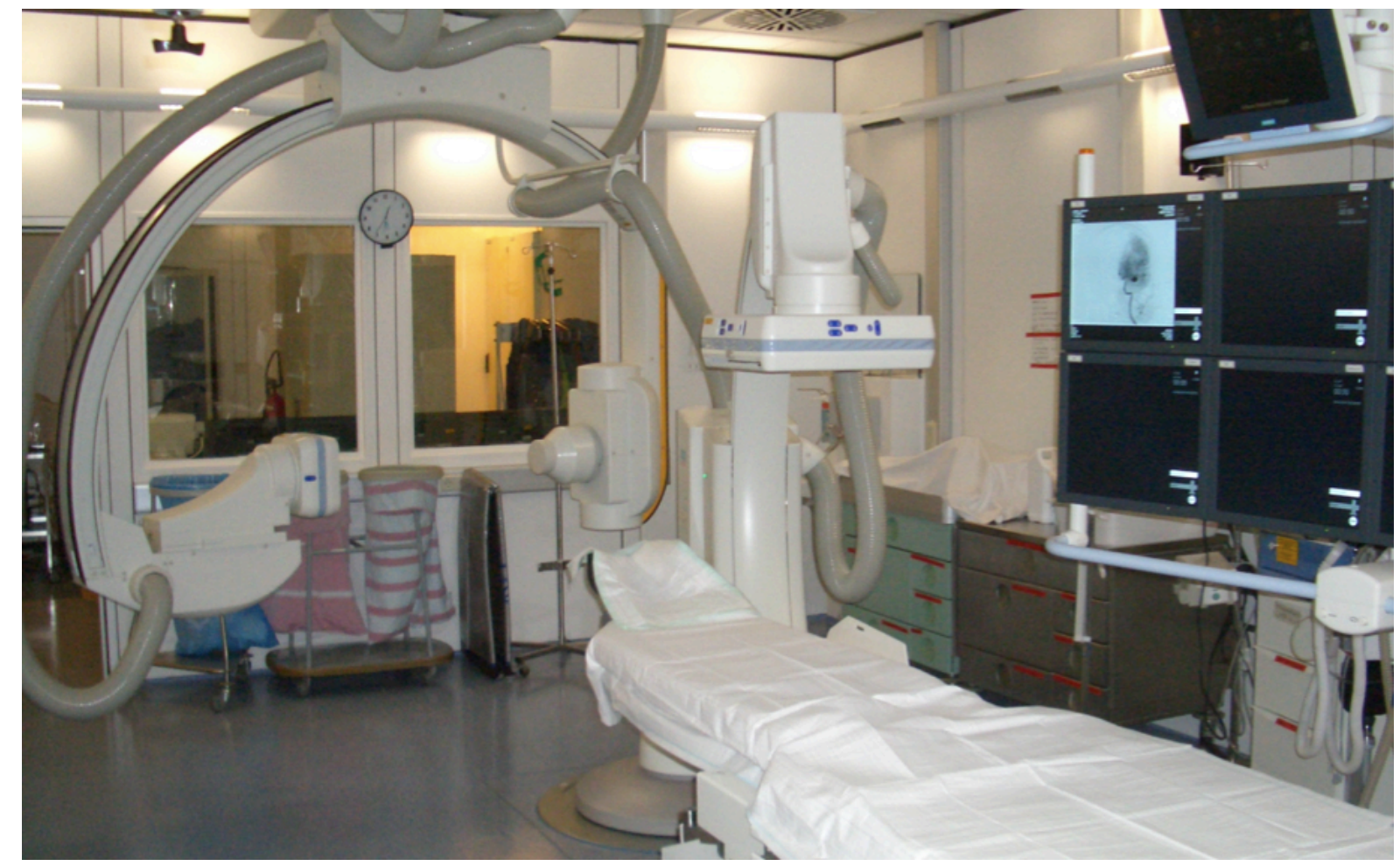

Abbildung 15 - Angiographiesystem „AXIOM Artis dBa” mit Flachdetektor-Technologie der Firma Siemens Healthcare (Erlangen, Deutschland) in der Abteilung für Neuroradiologie, Universitätsmedizin Göttingen.

\subsection{Ablauf der mechanischen Rekanalisation mit dem Penumbra System}

\subsubsection{Indikationen:}

Gemäß den Herstellerangaben ist das Penumbra System (PS) zur Rekanalisation / Revaskularisierung von Patienten mit akuten ischämischen Schlaganfällen, als Folge von Verschlusskrankheiten in den großen intrakraniellen Blutgefäßen (ICA, MCAAbschnitte M1 und M2, BA und VA), vorgesehen. Die Intervention sollte innerhalb von 8 Stunden nach Einsetzen der Symptome durchgeführt werden. Nach diesem Zeitfenster kann eine Intervention dennoch zu rechtfertigen sein, wenn in der Schlaganfall-Bildgebung (siehe Kapitel 1.2.5, Seite 16) ein signifikanter Gefäßverschluss und ein zu rettendes Penumbra-Areal dargestellt werden können, besonders bei Gefäßverschlüssen im vertebrobasilären Stromgebiet. Dennoch kann bei später Rekanalisation das Risiko für eine hämorrhagische Transformation oder sogar eine (symptomatische) intrakranielle Blutung steigen und einen negativen Einfluss auf das funktionelle Endergebnis des Patienten haben. 
Vor Beginn der mechanischen Rekanalisation muss die Sicherstellung der Behandlungsindikation eines ischämischen Schlaganfalles im intrakraniellen Gefäßsystem erfolgen und eine intrakranielle Blutung durch adäquate Diagnostik ausgeschlossen werden. Ausführliche Schlaganfall-Bildgebung (Schlaganfall-CT oder -MRT) sollte den Infarktkern, das umgebende Penumbra-Gewebe und den Gefäßverschluss zeigen. Dies ist besonders für Patienten außerhalb des IVTZeitfensters von 3 (bzw. 4,5) Stunden wichtig.
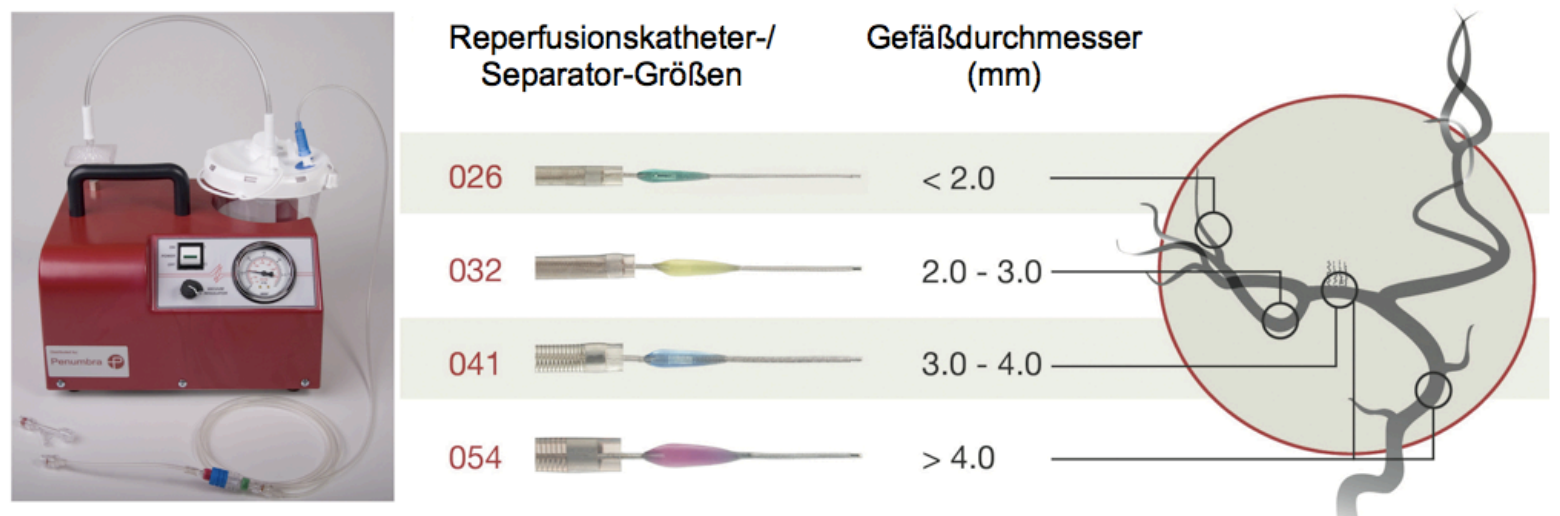

Abbildung 16 - Verfügbare Größen des Penumbra Systems. Die neurovaskulären Einsatzorte sind rechts dargestellt, wobei die Gefäßdurchmesser von Patient zu Patient variieren können. Mit freundlicher Abdruckgenehmigung von Penumbra Inc., Alameda, CA, USA.

\begin{tabular}{|c|c|c|c|c|c|}
\hline Beschreibung & $\begin{array}{c}\text { Proximaler } \\
\text { Außen- } \\
\text { durch- } \\
\text { messer }\end{array}$ & $\begin{array}{l}\text { Distaler } \\
\text { Außen- } \\
\text { durch- } \\
\text { messer }\end{array}$ & $\begin{array}{l}\text { Innen- } \\
\text { durch- } \\
\text { messer }\end{array}$ & $\begin{array}{l}\text { Gesamt- } \\
\text { länge }\end{array}$ & $\begin{array}{l}\text { Arbeits- } \\
\text { länge }\end{array}$ \\
\hline $\begin{array}{l}\text { Reperfusionskatheter } \\
026\end{array}$ & $\begin{array}{c}3.9 F \\
(0.051 \mathrm{in})\end{array}$ & $\begin{array}{c}2.8 \mathrm{~F} \\
(0.037 \mathrm{in})\end{array}$ & $\begin{array}{c}.026 \mathrm{in} \\
(0.66 \mathrm{~mm})\end{array}$ & $154 \mathrm{~cm}$ & $150 \mathrm{~cm}$ \\
\hline $\begin{array}{c}\text { Reperfusionskatheter } \\
031\end{array}$ & $\begin{array}{c}4.1 \mathrm{~F} \\
(0.054 \mathrm{in})\end{array}$ & $\begin{array}{c}3.4 \mathrm{~F} \\
(0.045 \mathrm{in})\end{array}$ & $\begin{array}{c}.032 \mathrm{in} \\
(0.81 \mathrm{~mm})\end{array}$ & $154 \mathrm{~cm}$ & $150 \mathrm{~cm}$ \\
\hline $\begin{array}{l}\text { Reperfusionskatheter } \\
041\end{array}$ & $\begin{array}{c}4.1 \mathrm{~F} \\
(0.054 \mathrm{in})\end{array}$ & $\begin{array}{c}4.1 \mathrm{~F} \\
(0.054 \mathrm{in})\end{array}$ & $\begin{array}{c}.041 \mathrm{in} \\
(1.04 \mathrm{~mm})\end{array}$ & $141 \mathrm{~cm}$ & $137 \mathrm{~cm}$ \\
\hline $\begin{array}{l}\text { Reperfusionskatheter } \\
054\end{array}$ & $\begin{array}{c}6.0 \mathrm{~F} \\
(0.080 \mathrm{in})\end{array}$ & $\begin{array}{c}5.0 \mathrm{~F} \\
(0.066 \mathrm{in})\end{array}$ & $\begin{array}{c}.054 \mathrm{in} \\
(1.37 \mathrm{~mm})\end{array}$ & $136 \mathrm{~cm}$ & $132 \mathrm{~cm}$ \\
\hline Separator 026 & k.A. & $\begin{array}{c}.022 \mathrm{in} \\
(0.56 \mathrm{~mm})\end{array}$ & k.A. & $200 \mathrm{~cm}$ & $155 \mathrm{~cm}$ \\
\hline Separator 032 & k.A. & $\begin{array}{c}.028 \mathrm{in} \\
(0.71 \mathrm{~mm})\end{array}$ & k.A. & $200 \mathrm{~cm}$ & $155 \mathrm{~cm}$ \\
\hline Separator 041 & k.A. & $\begin{array}{c}.035 \mathrm{in} \\
(0.89 \mathrm{~mm})\end{array}$ & k.A. & $200 \mathrm{~cm}$ & $142 \mathrm{~cm}$ \\
\hline Separator 054 & k.A. & $\begin{array}{l}.045 \mathrm{in} \\
(1.14 \mathrm{~mm})\end{array}$ & k.A. & $175 \mathrm{~cm}$ & $135 \mathrm{~cm}$ \\
\hline
\end{tabular}

Tabelle 5 - Technische Daten des Penumbra Systems. 


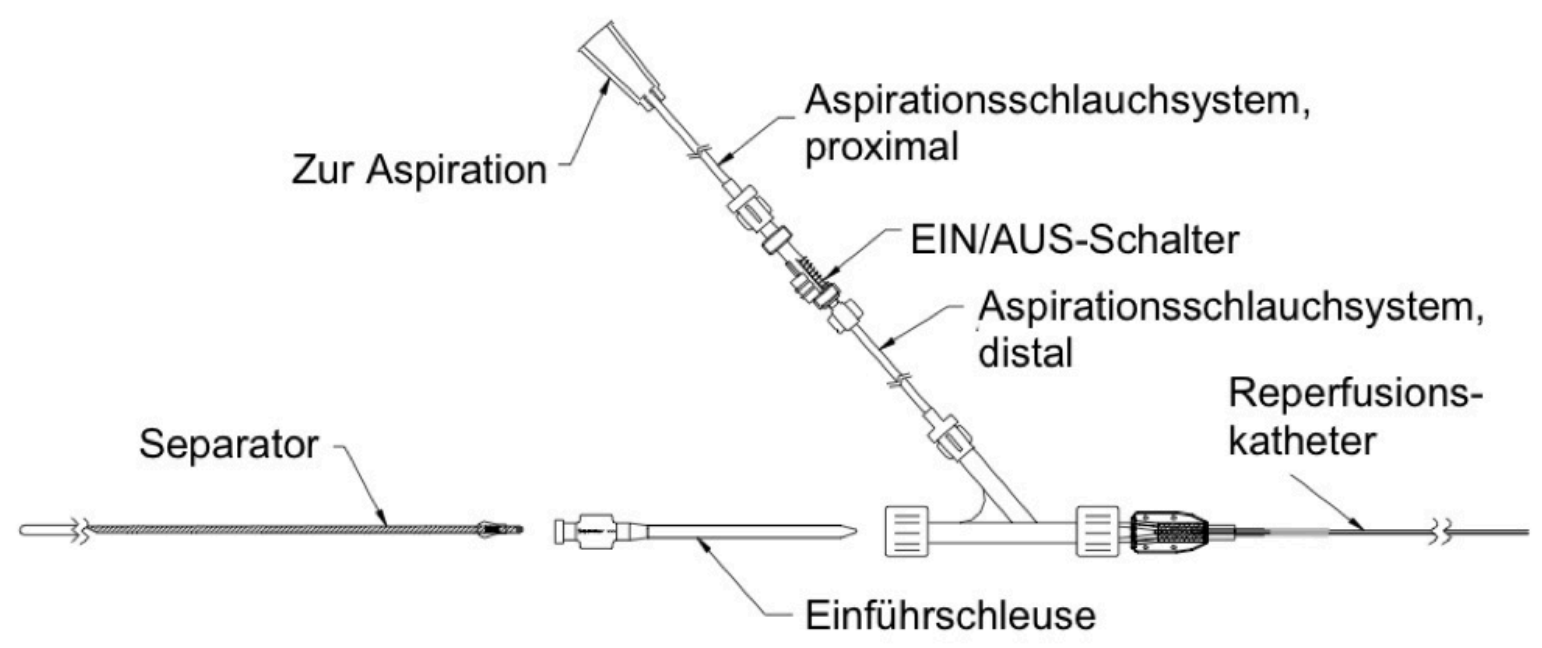

Abbildung 17 - Schematische Darstellung des Aufbaus des Penumbra Systems. Mit freundlicher Abdruckgenehmigung von Penumbra Inc., Alameda, CA, USA.

\subsubsection{Zugangstechniken}

Der Eingriff wird gewöhnlich in Intubationsnarkose vorgenommen, in bestimmten Einzelfällen kann auf eine Intubationsnarkose jedoch verzichtet und die Intervention in Sedierung durchgeführt werden.

Unter sterilen Kautelen erfolgt die perkutane Punktion der Arterie, präferentiell der rechten A. femoralis communis, nach Seldinger-Technik und das anschließende Einlegen einer transfemoralen 6F- oder 7F(French)-Schleuse.

Um den Ort des intrakraniellen Gefäßverschlusses zu erreichen, werden folgende Zugangstechniken in der vorderen bzw. hinteren Zirkulation angewandt:

- Vordere Zirkulation:

Kurze transfemorale 7F-Schleuse. Normalerweise wird ein 7F-Führungskatheter (z.B. Mach 1, Boston Scientific, Natick, MA, USA) mit normaler Konfiguration in der distalen CCA oder im zervikalen Segment der ICA positioniert. Dies ermöglicht die Verwendung aller verfügbaren PS-Größen. Der Penumbra Reperfusionskatheter (PRC) wird anschließend über einen Mikrodraht (z.B. Transend-14, Boston Scientific) an den Thrombus navigiert. Falls der PRC 054 zum Einsatz kommt, ist ein koaxiales Vorgehen mit einer Kombination von PRC 054 und PRC 032 hilfreich, um den Karotissiphon und den Abgang der A. ophthalmica zu passieren. Der koaxiale Gebrauch von PRC 054 und 032 bei distalen ICA- oder MCA-Verschlüssen im M1-Segment bietet den weiteren Vorteil, dass nach der proximalen Thrombusaspiration eventuell verbliebene distale Verschlüsse mit dem PRC 032 ohne Zeitverlust beseitigt werden können. 


\section{- Hintere Zirkulation:}

Kurze transfemorale 6F-Schleuse. Normalerweise wird ein 6F-Führungskatheter mit Vertebralis-Konfiguration (z.B. Envoy, Codman) im V2/V3-Übergang platziert.

Dies schließt den Gebrauch eines PRC 054 von vornherein aus. Bei Vorhandensein einer dominanten VA und geringer Gefäßschlängelung am VAAbgang kann auch ein 7F-Führungskatheter verwendet werden, dieser ermöglicht den Einsatz aller PRC-Größen. Der PRC wird ebenfalls mit einem Mikrodraht (z.B. Transend-14, Boston Scientific) zur Verschlusslokalisation geführt.

Vor Gebrauch und während der Intervention müssen die eingesetzten Katheter mit einer heparinisierten Kochsalzlösung $(0,9 \% \mathrm{NaCl})$ gespült werden, um thrombembolische Komplikationen zu vermeiden.

Ist ein Zugang zum thrombotischen Verschluss aufgrund einer weiter proximal vorgeschalteten hochgradigen Stenose (z.B. im Bereich der Carotis-Bifurkation) nicht möglich, ist ein Stenting zur Herstellung eines Zugangsweges nötig. Verwendet wurde hierfür normalerweise ein Stent mit Closed-Cell-Design (z.B. Carotid Wallstent, Boston Scientific).

\subsubsection{Rekanalisationsablauf}

Vor dem Start des Aspirationsmanövers werden standardmäßig diagnostische DSASerien durchgeführt, um Lokalisation und Ausmaß des Gefäßverschlusses und ggf. Kollateralen exakt darzustellen.

Das zu verwendende PS muss anhand der Verschlusslokalisation, des dortigen Gefäß-Innendurchmessers und ggf. vorhandener Gefäßschlängelung ausgesucht werden (siehe Abbildung 16 und Tabelle 5, Seite 52). Dabei sollte stets mit dem größtmöglichen PRC für das Zielgefäß begonnen werden, um einen maximalen Aspirationssog zu ermöglichen - dieser steigt mit der vierten Potenz des PRCInnendurchmessers nach dem Gesetz von Hagen-Poiseuille.

Nachdem der ausgewählte PRC entsprechend den zuvor beschriebenen Zugangswegen am Thrombus im zerebralen Gefäß platziert wurde (Abbildung 18 A), wird der neurovaskuläre Führungsdraht entfernt und stattdessen der Separator durch den PRC zur primären Okklusionsstelle vorgeführt (siehe Abbildung 17 und 18 A, Seiten 53 und 55). Der Separator als auch der PRC sind durch angebrachte Marker unter Röntgendurchleuchtung sichtbar, um eine präzise Steuerung zu ermöglichen. 
Für die Gefäßrekanalisation wird der Separator schließlich am proximalen Rand der primären Okklusion mehrfach durch den PRC vorgeschoben $(\sim 4 \mathrm{~mm})$ und zurückgezogen ( 20 mm) - dies geschieht unter kontinuierlichem Aspirationssog (-20 inHg / -677,2 mbar) durch die an den PRC angeschlossene Aspirationspumpe (siehe Abbildung 18 B-D). Während dieses Manövers dringt der Separator in den Thrombus ein, fragmentiert inn und erleichtert somit die Aspiration von Thrombusmaterial, gleichzeitig wird ein Verstopfen des PRC verhindert. Die Separator-Bewegungen müssen normalerweise mehrfach durchgeführt werden, um das gesamte thrombotische Material aspirieren zu können. Langstreckige oder mehrfache Gefäßverschlüsse erfordern oft einen Wechsel der PRC-Größen, abhängig von den Gefäß-Innendurchmessern. Die weiche Separator-Spitze ist nach einigen Aspirationsmanövern oft deformiert und muss dann durch einen neuen Separator ersetzt werden.

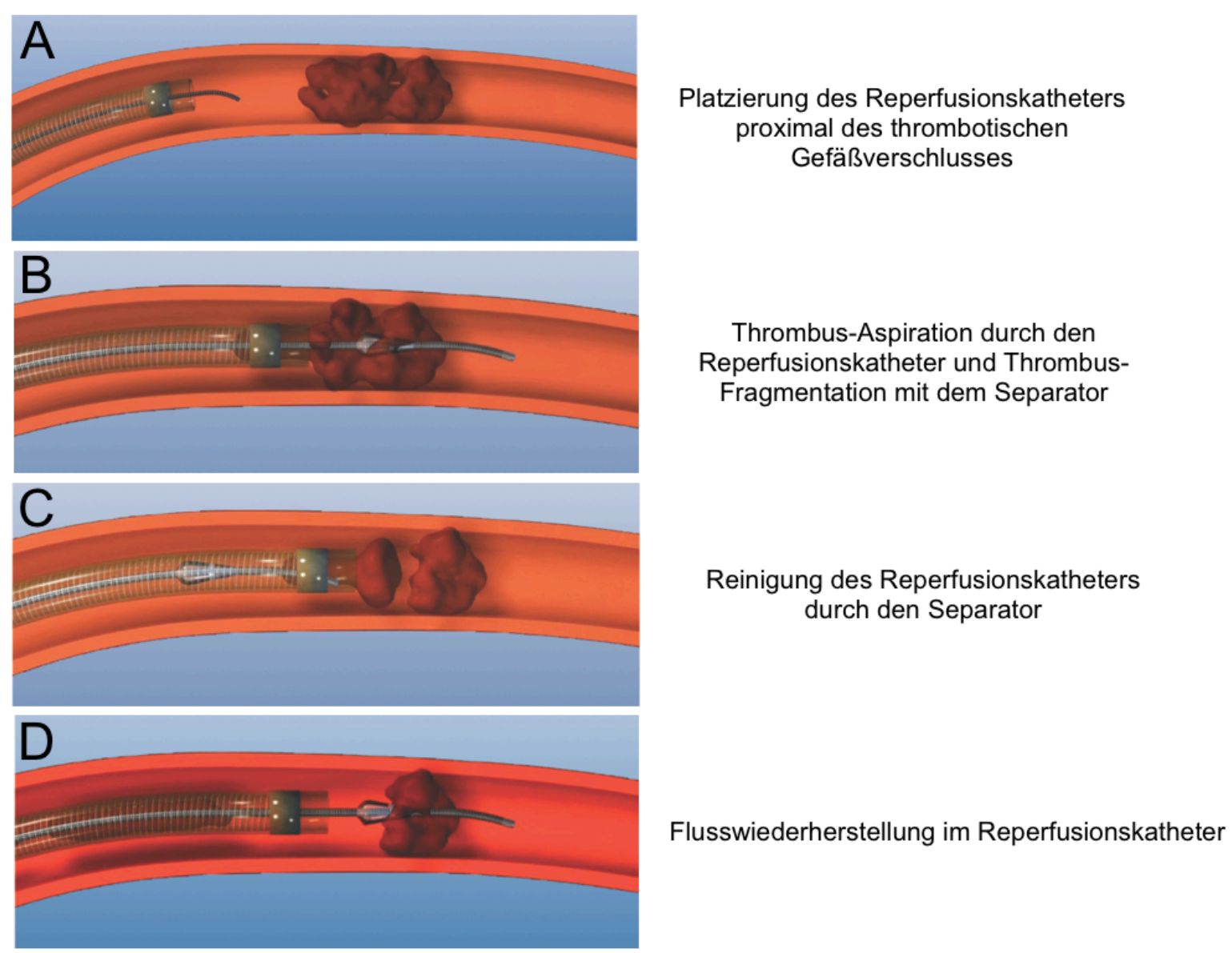

Abbildung 18 - Ablauf der mechanischen Rekanalisation mit dem Penumbra System. Mit freundlicher Abdruckgenehmigung von Penumbra Inc., Alameda, CA, USA. 
Nach dem Rekanalisationsmanöver können über den Führungskatheter diagnostische DSA-Serien aufgenommen werden, welche das Rekanalisationsergebnis zeigen.

Abschließend wurde bei allen Patienten am Ende der Prozedur eine ACT zum Ausschluss einer periinterventionellen Hämorrhagie durchgeführt.

Alle Interventionen der Abteilung für Neuroradiologie der Universitätsmedizin Göttingen wurden mit der digitalen Angiographie-Anlage mit Flachbild-Detektoren „Axiom Artis dTBA“ der Firma "Siemens Medical Solutions“ (Erlangen, Deutschland) durchgeführt (siehe Abbildung 15, Seite 51).

\subsection{Weiterbehandlung}

Im Anschluss an die Intervention wurden alle Patienten routinemäßig für mindestens drei Tage auf der neurologischen Intensivstation überwacht und danach auf die Stroke-Unit oder eine Normalstation verlegt.

Ein weiteres Kontroll-CT wurde 24 Stunden nach der Intervention durchgeführt oder auf jeden Fall bei einer weiteren neurologischen Verschlechterung zum Ausschluss einer $\mathrm{SICH}$.

Alle Patienten, bei denen zusätzlich zur mechanischen Rekanalisation eine Angioplastie oder ein Stenting durchgeführt wurde, bekamen oral lebenslang Acetylsalicylsäure (100 mg/Tag) und Clopidogrel $(75 \mathrm{mg} / \mathrm{Tag})$ für 6 Wochen nach der Prozedur verabreicht. Patienten mit einer Basilaris-Thrombose wurden heparinisiert und wurden nach Ermessen des behandeInden Neurologen antikoaguliert. Acetylsalicylsäure wurde allen anderen Patienten auf unbestimmte Zeit verschrieben, um weiteren kardiovaskulären Ereignissen vorzubeugen.

Vor Entlassung in die Rehabilitation wurde jeder Patient klinisch-neurologisch untersucht und eine Duplexsonographie veranlasst, um den klinischen Zustand und den zerebralen Gefäßstatus zu dokumentieren.

\subsection{Datenerfassung}

Für die klinisch-neurologische Ersteinschätzung und die weitere Verlaufsbeurteilung eines Schlaganfall-Patienten dienen verschiedene standardisierte und international anerkannte Skalensysteme. 
Die sich ergebenden Punktwerte für die in diese Studie eingeschlossenen Patienten wurden, sofern sie nicht routinemäßig bei der neurologischen Aufnahmeuntersuchung erfasst wurden, retrospektiv nachevaluiert. Als Grundlage dienten dafür neurologische Aufnahme-/Entlassungsbefunde, Notarztprotokolle sowie Entlassungsberichte der weiterbehandelnden Rehabilitationskliniken, welche im Zentralarchiv der Universitätsmedizin Göttingen verwahrt werden.

Alle retrospektiv erfassten Daten wurden von zwei für diese Evaluationen zertifizierten Untersuchern unabhängig voneinander begutachtet und beschließend nach dem Konsensusprinzip bewertet.

\subsubsection{NIH Stroke Scale (NIHSS)}

Der National Institutes of Health Stroke Scale wurde vom National Institutes of Neurological Disorders And Stroke für Schlaganfall-Therapiestudien konzipiert (Brott et al. 1989) und dient der systematischen, qualitativen Einschätzung des neurologischen Defizits eines Patienten nach einem akuten Schlaganfall (Spilker et al. 1997). Auf einer nicht-linearen Ordinalskala, welche von 0 bis maximal 42 Punkte reicht, wird die Schwere der neurologischen Ausfälle anhand von 13 Parametern beurteilt (Spilker et al. 1997).

Es konnte gezeigt werden, dass der NIHSS-Wert signifikant mit dem angiographischen Vorhandensein und der Lokalisation eines zerebralen Gefäßverschlusses (Fischer et al. 2005), sowie signifikant mit dem durch DWI- und PWIBilder ermittelten Infarktvolumen korreliert (Tong et al. 1998) und eine deutliche Vorhersage zu Genesungswahrscheinlichkeit/-verlauf des Patienten nach akutem Schlaganfall erlaubt (Adams et al. 1999; Heinemann et al. 1997).

Eine retrospektive Evaluation wird in der Literatur als zuverlässig und valide beschrieben (Kasner et al. 1999; Williams et al. 2000). Es zeigte sich jedoch in verschieden Studien, dass der NIHSS nur bei Anwendung durch qualifizierte/ zertifizierte Untersucher aussagekräftig und vergleichbar ist (Andre 2002; Goldstein et al. 1989; Josephson et al. 2006; Schmulling et al. 1998).

Im Anhang ist eine in der Abteilung für Neurologie der Universitätsmedizin Göttingen verwendete, modifizierte Version des NIHSS aufgeführt (siehe Anhang 1, Seite 101).

\subsubsection{Modified Rankin Scale (mRS)}

Der Modified Rankin Scale (mRS) ist ein weit verbreiteter Gradmesser für das globale funktionelle Outcome von Schlaganfall-Patienten (Quinn et al. 2009 a). Der mRS quantifiziert die Beeinträchtigungen der Aktivitäten des täglichen Lebens nach 
einem erlittenen Schlaganfall anhand einer 7-stufigen Ordinalskala von 0 (ohne Symptome) bis 5 (Patient bedarf ständiger Hilfe) bzw. 6 (Tod).

Ein $m R S$ von $\leq 2$ wird in der Schlaganfallforschung als gutes funktionelles Endergebnis gewertet und steht für eine geringgradige Funktionseinschränkung des Patienten, die inn im Alltag in die Lage versetzt, die eigenen Angelegenheiten ohne Hilfe zu erledigen.

Diese Skala wurde erstmals 1957 von Dr. John Rankin veröffentlicht (Rankin 1957) und war zunächst nicht für den Einsatz in klinischen Schlaganfall-Studien ausgelegt (Quinn et al. 2008). Später wurde der mRS durch das Oxfordshire Community Stroke Project (Bamford et al. 1989) um kognitive und sprachliche Beeinträchtigungen erweitert und wurde seitdem in vielen wegweisenden Studien zum Schlaganfall genutzt (Furlan et al. 1999; Hacke et al. 1995).

In zahlreichen Studien konnte gezeigt werden, dass eine Untersucherübereinstimmung gegeben ist, der mRS mit der Größe des betroffenen Hirnareals korreliert und eine konvergente Validität zu anderen Skalen, z.B. NIHSS, vorhanden ist. Somit eignet sich der mRS als Maß für die Wirksamkeit neuer Therapien des Schlaganfalls (Banks und Marotta 2007).

Oft wird die mRS-Skala noch dadurch vereinfacht, dass eine Dichotomisierung in mRS 0-2 versus 3-6 (im täglichen Leben unabhängig versus abhängig / verstorben) vorgenommen wird. Auf diesen Graduierungen des Behandlungserfolgs basieren die Ergebnisse der großen Akutbehandlungsstudien zum Schlaganfall.

Vergleichbare, aber nicht verwendete Skalen sind der Barthel Index (Granger et al. 1979; Mahoney und Barthel 1965) und der Glasgow Outcome Scale (Jennett und Bond 1975).

Im Anhang ist eine in der Abteilung für Neurologie der Universitätsmedizin Göttingen verwendete, modifizierte Version des mRS aufgeführt (siehe Anhang 2, Seite 102).

Für die Auswertung der Gefäßbildgebung vor und nach Intervention stehen nachfolgende Klassifikationssysteme zur Verfügung:

2.5.3 Modified Thrombolysis In Myocardial Infarction (mTIMI) Score Im Jahr 1985 entwickelte die Thrombolysis In Myocardial Infarction Study Group ein Graduierungssystem, das die Blutstromverhältnisse in den Koronararterien vor und nach Intervention qualitativ anhand der angiographischen Bilddaten erfasste (TIMI 
Study Group 1985; Sheehan et al. 1987). Diese Punkteskala reicht von 0 (keine Perfusion) bis 3 (vollständige Perfusion).

2001 veröffentlichten Qureshi et al. eine modifizierte TIMI-Skala (mTIMI), welche die intrakraniellen Gefäßverhältnisse berücksichtigt und heute ebenfalls in der neuroradiologischen Angiographie angewendet wird. Die modifizierte Punkteskala reicht von 0 (kein Fluss) bis 4 (distale Perfusion mit adäquater Perfusion in mehr als der Hälfte der distalen Gefäße). Mittlerweile existieren bis zu 7 Varianten der TIMISkala (Saver et al. 2009), in dieser Arbeit wird jedoch der mTIMI (Qureshi et al. 2001) verwendet.

Alle in der Vergangenheit erhobenen TIMI-Daten, ganz gleich ob basierend auf der Reperfusion, der Rekanalisation oder einer Mischung der beiden Aspekte, sind generell legitim und weitestgehend miteinander vergleichbar (Tomsick 2007).

Der TIMI-Score erwies sich als verwendbar für die Vorhersage des klinischen Ergebnisses nach einem Schlaganfall und intrakranieller Thrombolyse (Arnold et al. 2005).

Im Anhang ist die für diese Arbeit verwendete Skala des mTIMI aufgeführt (siehe Anhang 3, Seite 102).

\subsubsection{Thrombolysis in Cerebral Infarction (TICI) Score}

Das Technology Assessment Committee der American Society of Interventional and Therapeutic Neuroradiology stellte 2003 das TICl-Klassifikationssystem (Thrombolysis in Cerebral Infarction) vor, um ein einheitliches Standard-Maß für zukünftige Thrombolyse-Studien im zerebralen Gefäßkreislauf zu haben (Higashida et al. 2003). Im Gegensatz zur ursprünglichen TIMI-Klassifikation besteht der Unterschied in der Evaluation der Reperfusion im distal des Gefäßverschlusses befindlichen Gefäßterritoriums, nicht nur in der Betrachtung des Verschlussortes. Bisher wurde das TICl-Klassifikationssystem erst in wenigen Studien eingesetzt (Tomsick 2007) und wurde selbst noch keiner genaueren Evaluierung unterzogen.

Die Skala reicht von 0 (keine Perfusion) bis 3 (komplette Perfusion).

Im Anhang ist die für diese Arbeit verwendete Skala aufgeführt (siehe Anhang 4, Seite 102). 


\subsubsection{ECASS-Klassifikation intrakranieller Blutungen}

Intrakranielle Blutungen sind eine der bedeutendsten Komplikationen der thrombolytischen Therapie. Für die Einteilung der hämorrhagischen Ereignisse wurde auf die in den ECASS-Studien (European Cooperative Acute Stroke Study) verwendeten morphologischen Kriterien der Fiorelli-Klassifikation zurückgegriffen (Fiorelli et al. 1999; Hacke et al. 2008). Sie können in hämorrhagische Infarkte (HI) und parenchymale Hämatome $(\mathrm{PH})$ unterteilt werden.

Bei einem $\mathrm{HI}$ können entlang dessen Infarktgrenzen kleine Petechien (HI-1) oder mehr konfluierende Petechien (HI-2) innerhalb des Infarktbereiches ohne Verdrängungseffekt auftreten. Ähnlich werden bei PH zwei Subtypen unterschieden: Die Blutung kann $\leq 30 \%$ des Infarktes betreffen und lediglich einen leichten Verdängungseffekt ausüben (PH-1) oder $>30 \%$ des Infarktgebietes betreffen mit signikantem Verdrängungseffekt ( $\mathrm{PH}-2)$.

Im Anhang ist die für diese Arbeit verwendete Skala aufgeführt (siehe Anhang 5, Seite 103).

Eine symptomatische intrakranielle Blutung wurde definiert als das Vorhandensein einer nach ECASS definierten $\mathrm{ICH}$ im Kontroll-CT nach 24 Stunden und eine klinische Verschlechterung des NIHSS um $\geq 4$ Punkte oder ein Versterben des Patienten innerhalb von 24-36 Stunden nach der Schlaganfallbehandlung, maßgeblich aufgrund der Blutung.

\subsection{Datenauswertung}

Für die statistische Auswertung wurden die deskriptive Statistik, sowie Kontingenztabellen verwendet. Die Rekanalisationsergebnisse und neurologische Skalenwerte vor und nach der Behandlung wurden mit dem Matched-Pair-WilcoxonTest verglichen. Die Unterschiede der nominalen Variablen wurden mit dem ChiQuadrat-Test berechnet. Für kontinuierliche Variablen wurde der Mann-Whitney-Test angewendet. P-Werte $<0,05$ wurden als statistisch signifikant bewertet. Alle Ergebnisse wurden mit dem Statistical Package for the Social Sciences 17 (SPSS; Chicago, IL, USA), sowie Numbers (Apple Inc., Cupertino, CA, USA) berechnet. 


\subsection{Ethik}

Ein Votum der Ethik-Kommission der Medizinischen Fakultät der Georg-AugustUniversität zu Göttingen ist entsprechend den Richtlinien der Ethik-Kommission nicht erforderlich.

Da es sich um eine retrospektive epidemiologische Studie ohne personenbezogene Daten handelt, das Forschungsvorhaben der Qualitätssicherung dient und ohne zusätzlich belastende Untersuchungen für die Patienten auskommt, musste kein Votum der Ethik-Kommission eingeholt werden. 


\section{Ergebnisse}

\subsection{Patientenkollektiv und präinterventionelle Klinik}

\subsubsection{Alters- und Geschlechtsverteilung}

Diese Arbeit erfasst 91 Patienten mit symptomatischen, ischämischen Schlaganfällen mit einem mittleren Alter von 61,97 Jahren zum Zeitpunkt der Intervention, der Median lag bei 69 Jahren. Die Spannweite des Patientenkollektives betrug 3 - 98 Jahre.

Die Patientenpopulation setzt sich aus 48 Männern (52,7 \%) und 43 Frauen (47,3 \%) zusammen.

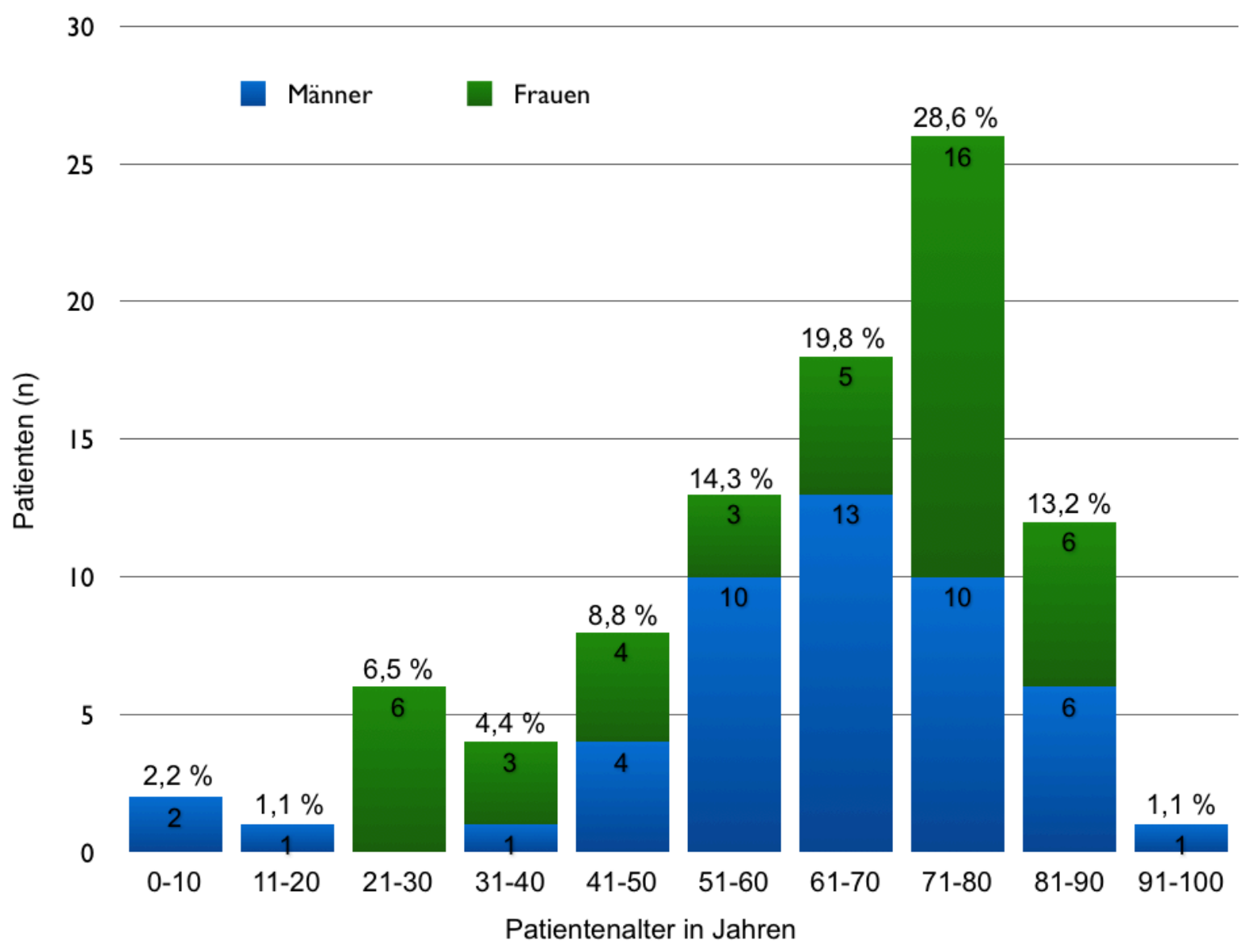

Abbildung 19 - Alters- und Geschlechtsverteilung des behandelten Patientenkollektivs.

\subsubsection{Präinterventionelle Diagnostik und neurologische Klinik}

Bei Klinikaufnahme zeigten die Patienten in der initialen neurologischen Untersuchung einen durchschnittlichen NIHSS-Wert von 17 (SD $\pm 6,1$ ), während der mittlere mRS bei Aufnahme bei $4,48(S D \pm 0,72)$ lag. 
Insgesamt 47 Patienten (52 \%) hatten bei Aufnahme einen NIHSS-Punktwert von $\geq 20$ oder einen BA-Verschluss, also sehr schwerwiegende Schlaganfälle.
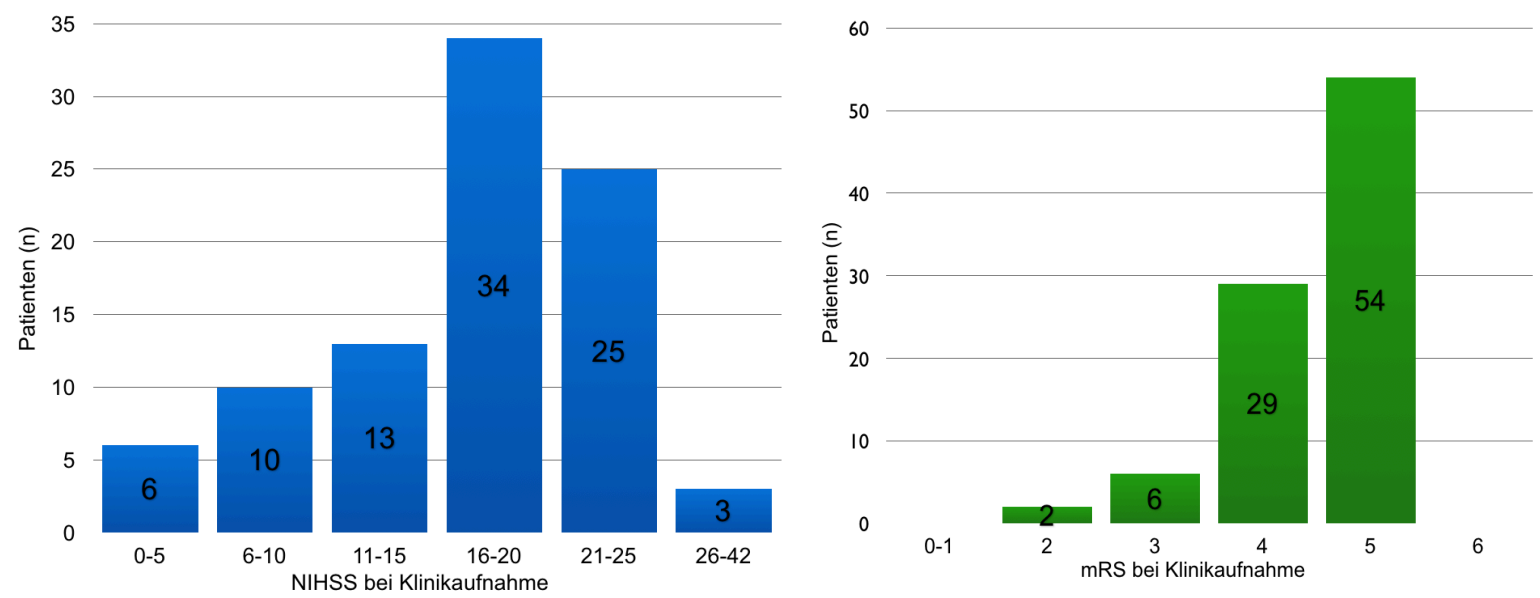

Abbildung 20 (links) - Verteilung der präinterventionellen Graduierungspunkte des NIHSS. Abbildung 21 (rechts) - Verteilung der präinterventionellen Graduierungspunkte des mRS.

\subsubsection{Präinterventionelle Bildgebung}

Vor dem Therapiestart wurde bei allen Patienten eine ausführliche Bildgebungsdiagnostik des Kopfes durchgeführt. Eine intrakranielle Blutung wurde ausgeschlossen. Die Mehrzahl der Patienten bekam ein CCT, bei sehr jungen Patienten wurde ein kranielles MRT angefertigt.

\subsubsection{Zeitverlauf}

Durchschnittlich vergingen $154 \pm 244$ Minuten von Symptombeginn bis zur Klinikaufnahme mit einem Median von 79,5 Minuten. Nach Aufnahme ins Akutkrankenhaus dauerte es im Durchschnitt weitere $169 \pm 93$ Minuten, bis die neuroradiologische Intervention mit der Punktion der Leistenarterie begonnen wurde (Median: 153 Minuten).

Im Median verstrich bei Verschlüssen in der vorderen Zirkulation weniger Zeit bis zum Interventionsbeginn im Vergleich mit Okklusionen in der hinteren Zirkulation (227 vs. 260 Minuten), wobei die Rekanalisation in der vorderen Zirkulation länger dauerte (53 vs. 41 Minuten).

So vergingen durchschnittlich $312 \pm 341$ Minuten von Symptombeginn bis Interventionsstart (Median: 196 Minuten). 
Vordere Zirkulation

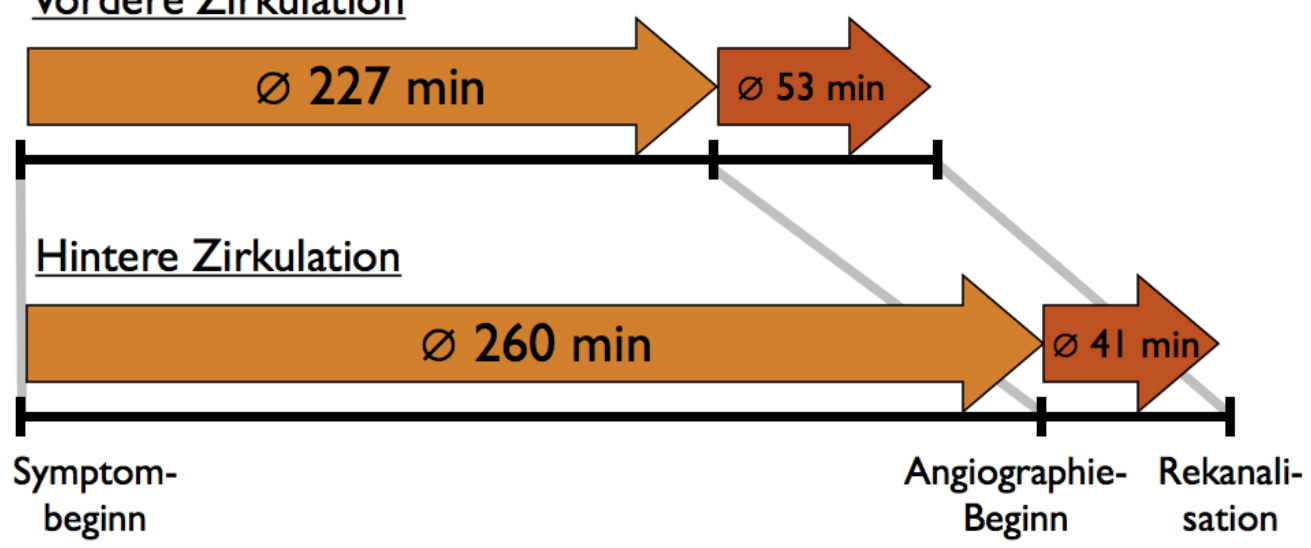

Abbildung 22 - Zeitverlauf von Symptombeginn bis Gefäßrekanalisation für die vordere und hintere Zirkulation.

\subsection{Interventionen}

\subsubsection{Intrakranielle Gefäßverschlüsse}

\subsubsection{Präinterventioneller Gefäßstatus}

Insgesamt 91 Patienten mit symptomatischen, behandlungsbedürftigen Gefäßverschlüssen wurden für die mechanische Rekanalisation im Rahmen einer interventionellen Therapie vorgesehen.

\begin{tabular}{|c|c|c|c|}
\hline \multicolumn{4}{|c|}{ Präinterventioneller Gefäßstatus } \\
\hline \multicolumn{2}{|c|}{$\mathrm{TICl}$} & \multicolumn{2}{|c|}{ mTIMI } \\
\hline Grad & $\mathrm{n}(\%)$ & Grad & $\mathrm{n}(\%)$ \\
\hline 0 & $65(71,42 \%)$ & 0 & $63(69,23 \%)$ \\
\hline 1 & $26(28,57 \%)$ & 1 & $20(21,98 \%)$ \\
\hline $\begin{array}{c}2 \\
2 a \\
2 b\end{array}$ & $\begin{array}{l}0(0 \%) \text { * } \\
0(0 \%) \text { * } \\
0(0 \%) \text { * }\end{array}$ & 2 & $8(8,79 \%)$ \\
\hline 3 & $0(0 \%) *$ & 3 & $0(0 \%)$ \\
\hline * aus & chlossen & 4 & $0(0 \%)$ \\
\hline
\end{tabular}

Tabelle 6 - Präinterventioneller Gefäßstatus der Patienten entsprechend den Graduierungssystemen TICI und mTIMI. 
In der DSA-Bildgebung zu Beginn der Intervention wurde bei 65 Patienten (71\%) ein TICl-Wert von 0 und bei 26 (29\%) Patienten ein TICl-Wert von 1 angiographisch festgestellt. Dies entspricht vollständigen Gefäßverschlüssen der intrakraniellen Hirngefäße mit keiner oder minimaler Perfusion im distal gelegenen Gefäßbett. Alle Gefäßverschlüsse waren somit hämodynamisch relevant.

\subsubsection{Lokalisation der Gefäßverschlüsse}

72 der Gefäßverschlüsse $(79,1 \%)$ befanden sich in der vorderen Zirkulation, wobei 39 MCA- (42,9 \%) und 21 Carotis-T-Verschlüsse $(23,1 \%)$ den Hauptanteil darstellten. 11 akute Okklusionen in der ICA und ein Verschluss in der CCA wurden therapiert.

Bei den 19 (20,9 \%) festgestellten Gefäßverschlüssen in der hinteren Zirkulation machten 15 Basilarisverschlüsse (16,5 \%) den Großteil aus. 3 weitere Verschlüsse bestanden aus kombinierten VA- und BA-Okklusionen, ein Gefäßverschluss befand sich in der PCA.

Die Abbildungen 23 und 24 stellen die Verteilung der 91 Gefäßverschlüsse des Patientenkollektives dar, jeweils entsprechend den Gefäßlokalisationen und der Hemisphärenseite.
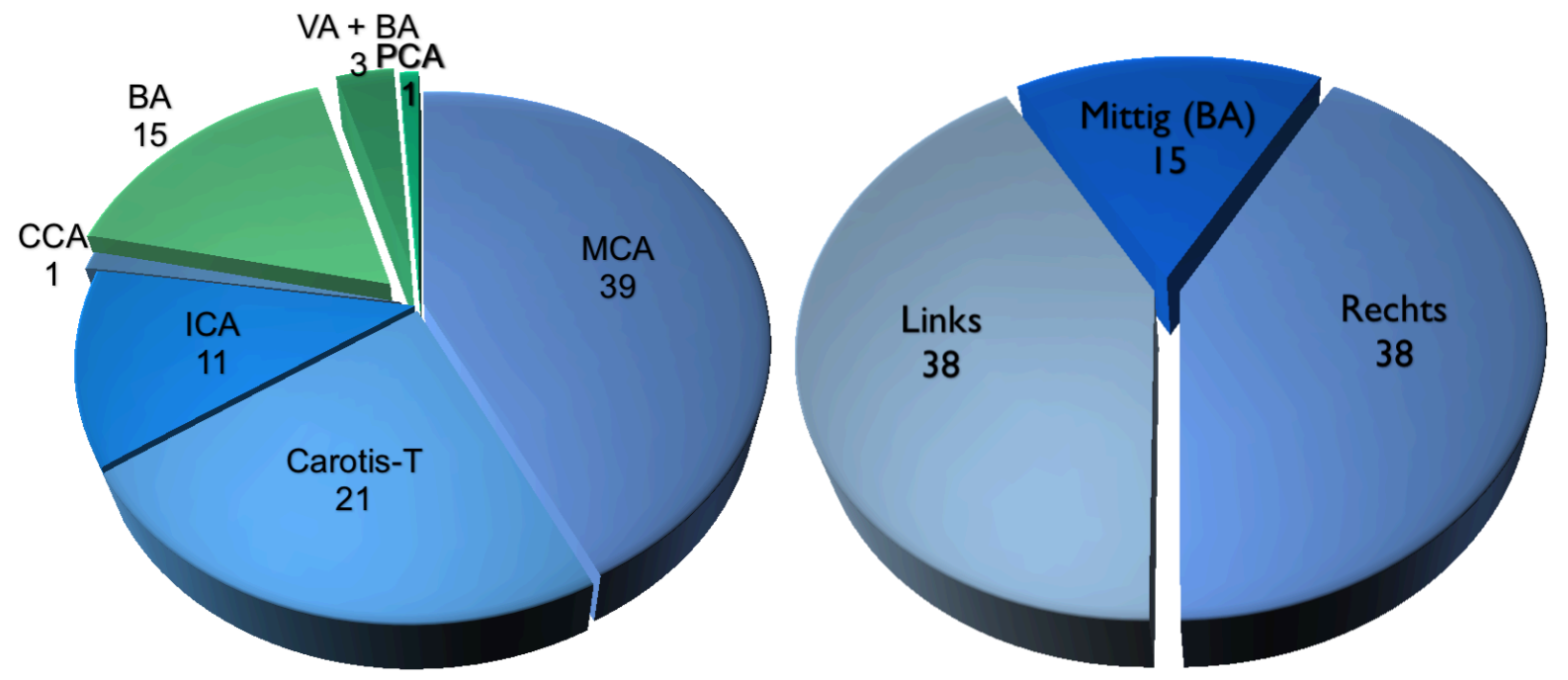

Abbildung 23 (links) - Übersicht über die Lokalisationen der Gefäßverschlüsse.

Abbildung 24 (rechts) - Übersicht über die Seitenverteilung der Gefäßverschlüsse auf die Hemisphären. 


\subsubsection{Materialeinsatz}

Bei allen Interventionen wurde das Penumbra System für die mechanische Rekanalisation der Hirngefäße eingesetzt. Als erfolgter Einsatz wurde dabei die Initiation der Aspiration und der Einsatz des Separators gewertet.

Im Durchschnitt wurden im Patientenkollektiv pro Intervention 1,49 Penumbra Reperfusionskather verwendet. In einigen Fällen musste während der Intervention der Separator des Penumbra Systems gegen einen neuen ausgetauscht werden, da sich die fragile Spitze aufgrund der Thrombusbeschaffenheit verformt hatte.

Am häufigsten zum Einsatz kann das Penumbra System der Größe 041, welches in $62,9 \%$ der Interventionen verwendet wurde und bis zum M1-Segment der MCA vordringen kann. Der Aspirationskatheter 054, welchen eine besonders hohe Aspirationsrate charakterisiert, wurde bei weiter proximalen ICA-, CCA- und BAVerschlüssen benutzt (7,8 \%). Die verfügbaren Größen $032(26,7 \%)$ und 026 $(2,6 \%)$ zeigten sich nützlich bei der Rekanalisation von Gefäßsegmenten distal des M1-Abschnittes, entweder für primäre Verschlüsse oder im Fall von distal aufgetretenen Emboli.

Bei insgesamt 27 Patienten wurden zusätzlich Stents $(n=31)$ für die Flusswiederherstellung verwendet, entweder zur Herstellung eines Zugangsweges aufgrund abgangsnaher Stenosen der Kopf-/Halsgefäße (20 Fälle) oder zur Therapie von primären oder intraprozedural aufgetretenen Dissektionen (7 Fälle). Bei 4 Patienten war die Implantation von 2 Stents notwendig, um ein zufriedenstellendes Ergebnis zu erzielen.

Zur Anwendung kam 28 Mal der Carotid Wall Stent des Herstellers Boston Scientific (Natick, MA, USA) in verschiedenen Größen (7x30 mm, 7x40 mm, 7x50 mm, 8x21 mm, 8x29 mm 8x36 mm, 10x40 mm). In zwei Fällen wurde der Cordis Enterprise Stent (Cordis Neurovascular Inc., Miami, FL, USA) in der Größe 4,5x28 mm verwendet, ein weiteres Mal der Driver Stent $(4 \times 18 \mathrm{~mm})$ von Medtronic (Minneapolis, MN, USA).

\subsubsection{Kombinationstherapien \& rt-PA-Einsatz}

Im beschriebenen Patientenkollektiv von 91 Patienten wurde bei 14 Interventionen $(15,7 \%)$ einzig das Penumbra System zur mechanischen Rekanalisation der Hirngefäße eingesetzt. Bei den weiteren 77 Eingriffen wurde die mechanische Lyse 
mit intravenöser (11,2\%), intraarterieller (29,2 \%) oder kombinierter intravenöser und intra-arterieller medikamentöser Lyse (43,8 \%) mittels rt-PA kombiniert.

Bei 2 Interventionen konnte die Lysekombination nicht aus den zur Verfügung stehenden Daten ermittelt werden.

Insgesamt betrachtet wurden durchschnittlich $37,7 \pm 26,3 \mathrm{mg}$ rt-PA pro Patient eingesetzt. Der Median lag bei $40 \mathrm{mg}$ rt-PA pro Patient. Intraarteriell wurden im Durchschnitt 32,2 \pm 22,4 mg rt-PA eingesetzt (Median: $34 \mathrm{mg}$ ), intravenös 27,8 \pm 18,0 mg rt-PA (Median: $20 \mathrm{mg}$ ).

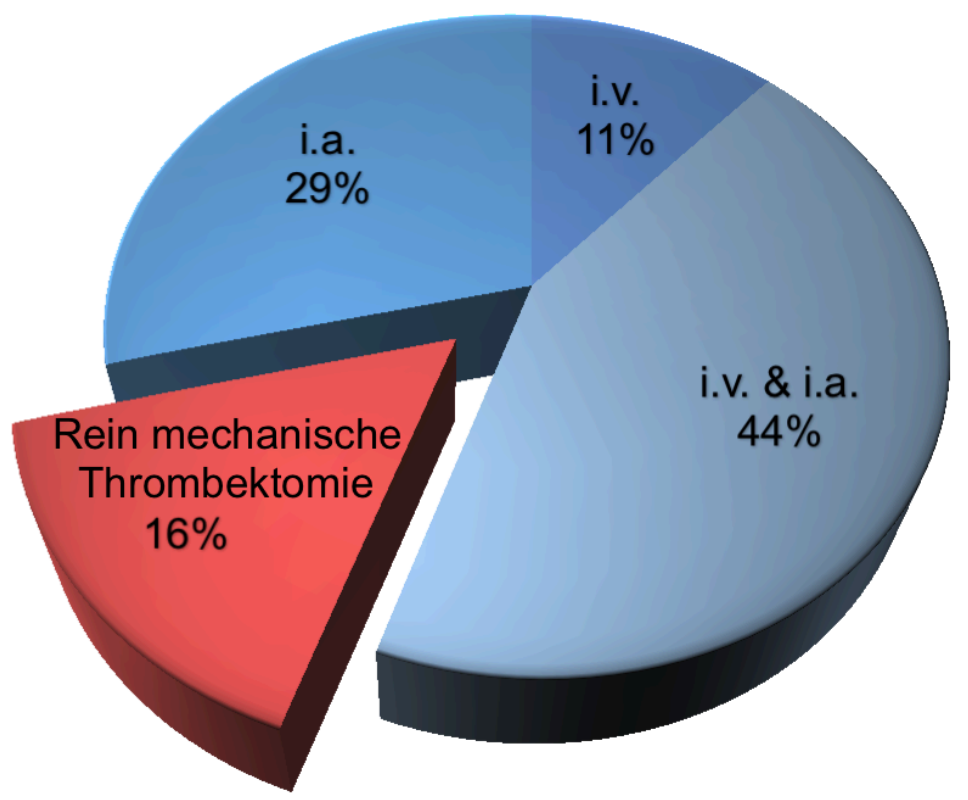

Abbildung 25 - Häufigkeiten des Einsatzes von intravenöser (i.v.) und intraarterieller (i.a.) Fibrinolyse sowie reiner mechanischer Thrombektomie.

\subsubsection{Prozedurale Komplikationen}

\subsubsection{Intraprozedurale Komplikationen und Todesfälle}

Bei sieben Interventionen $(7,7 \%)$ traten während des Eingriffs hämodynamisch relevante Vasospasmen auf, die alle durch medikamentöse Therapie mit Nimodipin (Carinopharm $\mathrm{GmbH}$, Elze, Deutschland) in einer Dosis von bis zu 2 mg erfolgreich behandelt werden konnten. Dies ist somit die am häufigsten beobachtete Nebenwirkung der Aspirationsthrombektomie beziehungsweise der zerebrovaskulären Katheternavigation.

Bei vier weiteren Patienten (4,4 \%) traten Gefäßdissektionen auf. Alle Dissektionen befanden sich in der ICA und wurden im Rahmen der Intervention mit jeweils einem Carotid Wingspan Stent (Boston Scientific, Natick, MA, USA) erfolgreich therapiert.

Leistenhämatome an der arteriellen Punktionsstelle wurden bei 2 Patienten $(2,2 \%)$ dokumentiert. Ein Patient hatte einen Einriss der A. iliaca externa rechts, was zu einem großen retroperitonealen Hämatom mit hypovolämischem Schock führte und 
operativ korrigiert werden musste. Der zweite Patient zeigte postinterventionell ein Aneurysma der rechten A. iliaca mit einer $\mathrm{Hb}$-relevanten Einblutung in den Retroperitonealraum und die Bauchwand. Er benötigte 3 Erythrozytenkonzentrate und eine Übernähung des Aneurysmas.

In 2 Fällen (2,2 \%) kam es zu einem Bruch der Separator-Spitze. Die Bruchstelle lag jeweils am Übergang vom harten proximalen zum weicheren mittleren Drahtanteil. Das gesamte Material konnte in beiden Fällen erfolgreich geborgen werden und es verblieb kein Material im Patienten.

Intraprozedural kam es im beschriebenen Patientenkollektiv zu keinem Todesfall. Somit war kein Todesfall unmittelbar nachweislich auf den Einsatz des Penumbra Systems zurückzuführen.

\subsubsection{Postprozedurale Komplikationen und Todesfälle}

Im direkt postinterventionell durchgeführten Flachdetektor-CT wurde bei einem Patienten eine rechts basal lokalisierte Subarachnoidalblutung festgestellt. Bei fehlendem Hinweis für ausgeprägte Vasospasmen war keine weitere spezifische Therapie im Verlauf notwendig.

Bei allen Patienten wurden postinterventionell nach 24 Stunden routinemäßig Schnittbildkontrollen durchgeführt. Bei 19 Patienten (20,9\%) zeigten sich dabei intrakranielle Blutungen ( $\mathrm{ICH}), 2$ davon waren Subarachnoidalblutungen. 5 der insgesamt detektierten Blutungen (5,5\%) wurden definitionsgemäß als symptomatische intrakranielle Blutung $(\mathrm{SICH})$ eingestuft.

Bei Anwendung der ECASS-Klassifikation für ICH (siehe Anhang 5, Seite 103) wurden 5 Fälle als hämorrhagischer Infarkt Typ 1 (HI-1), 3 Fälle als hämorrhagischer Infarkt Typ 2 (HI-2), 5 Fälle als parenchymatöses Hämatom Typ 1 (PH-1) und 4 Fälle als parenchymatöses Hämatom Typ 2 (PH-2) bewertet.

Bei der weitergehenden statistischen Analyse ergab sich kein statistisch signifikanter Zusammenhang zwischen dem Auftreten einer $\mathrm{ICH}$ und der frühen bzw. späten Gefäßrekanalisation $(P=0.29)$. Des Weiteren waren die $\mathrm{ICH}$ unabhängig vom Rekanalisationsergebnis $(P=0.38)$. Ebenso konnte kein signifikanter Zusammenhang zwischen der rt-PA-Applikation und dem Auftreten von $\mathrm{ICH}$ beobachtet werden $(P=0.58)$. 


\begin{tabular}{|c|c|c|c|c|c|c|c|}
\hline \multicolumn{8}{|c|}{ Postinterventionelle intrakranielle Blutungen } \\
\hline $\begin{array}{c}\text { ICH- } \\
\text { Klassifikation } \\
\text { nach ECASS }\end{array}$ & $\begin{array}{c}\text { Patient } \\
\text { Nr. }\end{array}$ & $\begin{array}{l}\text { Ver- } \\
\text { schluss- } \\
\text { ort }\end{array}$ & $\begin{array}{l}\text { Klinische } \\
\text { Ver- } \\
\text { schlech- } \\
\text { terung? }\end{array}$ & $\begin{array}{l}\text { NIHSS bei } \\
\text { Aufnahme }\end{array}$ & $\begin{array}{l}\text { rt-PA } \\
\text { in } \mathrm{mg}\end{array}$ & $\begin{array}{l}\mathrm{TICl} \\
\text { post }\end{array}$ & $\begin{array}{c}\text { NIHSS } \\
\text { nach } 90 \\
\text { Tagen/ } \\
\text { Reha }\end{array}$ \\
\hline \multirow{5}{*}{$\mathrm{HI}-1$} & 19 & M1 & nein & 20 & - & 3 & n.v. \\
\hline & 25 & M1 & nein & 5 & 30 & 3 & 1 \\
\hline & 45 & M2 & nein & 10 & 40 & 3 & 8 \\
\hline & 69 & M1 & nein & 15 & 63 & $2 b$ & 16 \\
\hline & 81 & BA & nein & 13 & 20 & $2 a$ & 8 \\
\hline \multirow{3}{*}{ HI-2 } & 35 & C7/M1 & nein & 9 & 8 & $2 b$ & 10 \\
\hline & 50 & M1 & nein & 18 & 10 & 3 & 5 \\
\hline & 78 & M1 & nein & 18 & 20 & 3 & 15 \\
\hline \multirow{5}{*}{$\mathrm{PH}-1$} & 22 & M1 & nein & 19 & 38 & $2 a$ & 12 \\
\hline & 52 & C7/M1 & nein & 22 & 65 & 3 & 9 \\
\hline & 63 & C7/M1 & nein & 14 & - & 3 & 4 \\
\hline & 73 & M1 & ja & 25 & 70 & 3 & 16 \\
\hline & 79 & M1 & nein & 23 & 100 & 3 & $\dagger$ \\
\hline \multirow{4}{*}{$\mathrm{PH}-2$} & 6 & C7/M1 & ja & 15 & 20 & $2 a$ & n.v. \\
\hline & 36 & C7/M1 & ja & 16 & 60 & $2 a$ & $\dagger$ \\
\hline & 62 & BA & ja & 24 & 40 & 3 & $\dagger$ \\
\hline & 82 & M1 & ja & 20 & 60 & $2 b$ & n.v. \\
\hline
\end{tabular}

Tabelle 7 - Postinterventionell aufgetretene intrakranielle Blutungen.

n.v.: nicht verfügbar

Bis auf zwei Ausnahmen befanden sich alle nach ECASS-Kriterien klassifizierten intrakraniellen Blutungen bei Patienten mit Gefäßverschlüssen im Bereich der proximalen Arteria cerebri media bzw. des Carotis-T. Symptomatische intrakranielle Blutungen entsprachen in 4 von 5 Fällen einem parenchymatösem Hämatom Typ 2, zwei dieser Patienten verstarben an den Folgen der Hämmorrhagisierung.

\subsubsection{Vaskuläre Ergebnisse}

\subsubsection{Postinterventioneller Gefäßstatus}

Durch den Therapieansatz einer neuroradiologischen Gefäßrekanalisation konnte bei 70 Patienten (76,9 \%) eine partielle oder vollständige Reperfusion, entsprechend $\mathrm{TICl} 2$ oder 3, erzielt werden.

Die Zahl derer Patienten, die postinterventionell einen TICI-Wert von 0 oder 1 zeigten, konnte auf $8(8,8 \%)$ und $13(14,3 \%)$ reduziert werden. Weitere $21(23,1 \%)$ hatten einen TICI-Wert von 2a, 12 (13,2 \%) einen TICI-Wert von $2 b$ und schließlich 37 (40,7\%) Patienten einen TICI-Wert von $3(P<0.001)$. 
Die folgende Abbildung 26 stellt die Verteilung der Rekanalisationsergebnisse insgesamt dar.

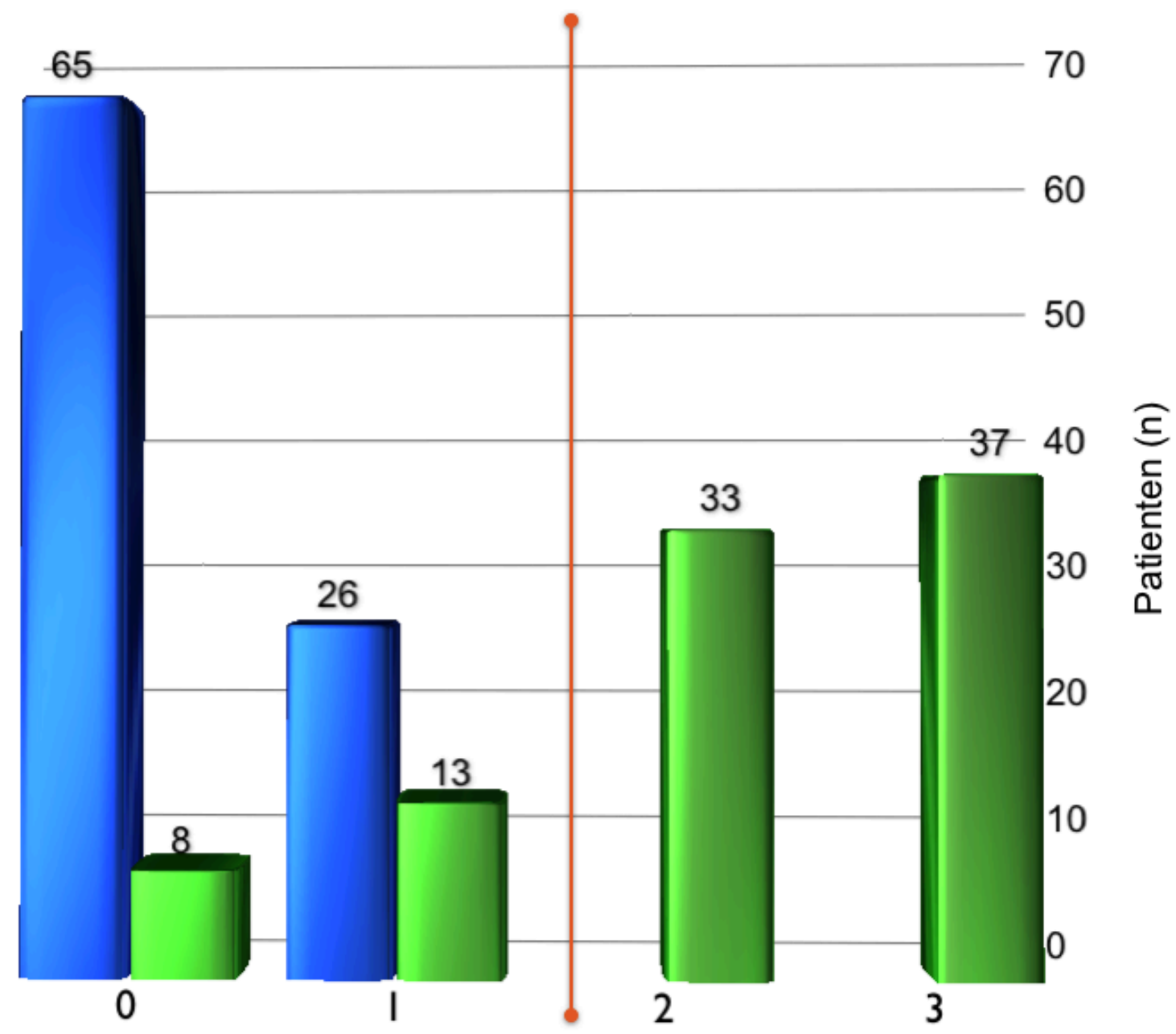

TICl-Werte

\section{vor Intervention $\quad$ nach Intervention $\quad P<0.001$}

Abbildung 26 - Prä- und postinterventioneller Gefäßstatus des Patientenkollektivs. TICIGrad 2 und 3 werden als erfolgreiche Rekanalisation gewertet (rechts der roten Trennlinie).

Betrachtet man die Rekanalisationserfolgsrate anhand der Verschlusslokalisationen, wurde eine partieller oder vollständiger Reperfusion in folgender Häufigkeit erzielt: In $79,5 \%$ (31/39) der MCA-Verschlüsse; in 85,7 \% (18/21) der Carotis-T-Verschlüsse; in 63,6 \% (7/11) der ICA-Okklusionen; in 1 (100\%) Fall mit CCA-Verschluss und in der hinteren Zirkulation in 66,7 \% (10/15), $100 \%(3 / 3)$ und $0 \%(0 / 1)$ der BA-, kombinierten VA \& BA-Verschlüsse und PCA-Verschlüsse $(P=0.27)$. Demzufolge wurde ein TICl-Wert von 2 oder 3 in 79,2 \% der Okklusionen in der vorderen Zirkulation und in 68,4 \% der Verschlüsse in der hinteren Zirkulation erreicht. 


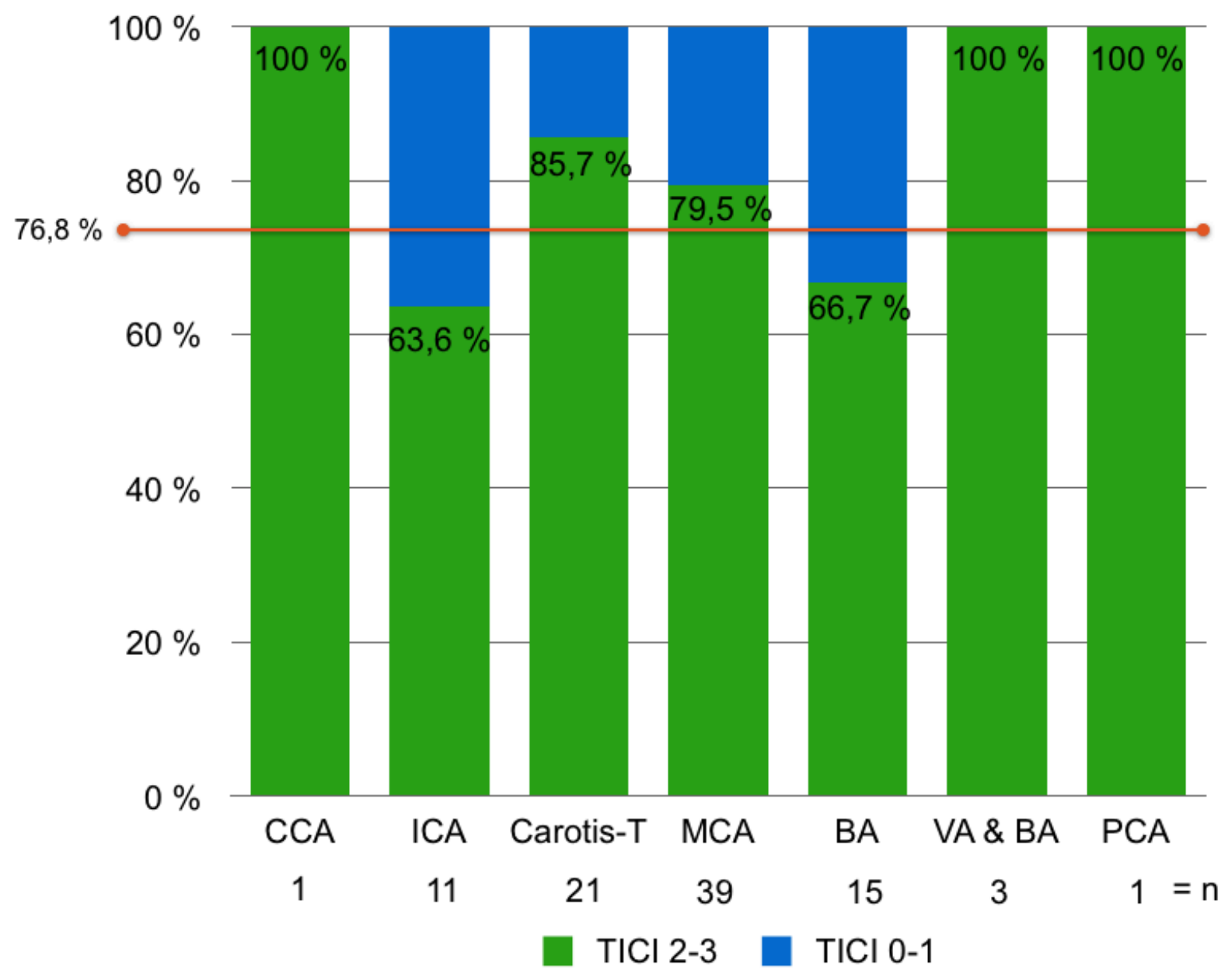

Abbildung 27 - Postinterventionelle Rekanalisationsraten nach Verschlusslokalisation. Die rote Linie kennzeichnet die mittlere Rekanalisationsrate von 76,8 \% im Gesamtkollektiv.

\subsubsection{Rekanalisationserfolg nach Kombinationstherapien}

Betrachtet man den Rekanalisationserfolg entsprechend den in Kapitel 1.3.3.4 (Seite 33) aufgezeigten Kombinationstherapien, ergibt sich folgendes Ergebnis:

Bei 14 Patienten wurde lediglich die mechanische Rekanalisation mittels des Penumbra Systems eingesetzt und führte in 11 Fällen (78.6 \%) zu einer erfolgreichen Gefäßwiedereröffnung ( $\mathrm{TICl} 2-3$ ). Wurde eine Bridging-Lyse intravenös vor der Intervention durchgeführt, lag die Erfolgsrate bei $60 \%(6 / 10)$, bei lediglich intraarterieller rt-PA-Gabe bei 84,6 \% (22/26). In 39 Fällen, bei denen neben der mechanischen Rekanalisation sowohl intravenös, als auch intraarteriell rt-PA appliziert wurde, konnte in $74,4 \%(29 / 39)$ eine partielle oder vollständige Reperfusion ( $\mathrm{TICl} 2-3$ ) erzielt werden. In dieser Kohorte zeigte sich bei Patienten mit einer Gesamtdosis von $\leq 20$ mg rt-PA (i.v. \& i.a.) mit einer Rekanalisationsrate von $83,3 \%$ ein etwas besseres Ergebnis als bei einer rt-PA-Applikation von $>20 \mathrm{mg}$ $(72,7 \%)(P=0.35)$. 


\subsubsection{Zeitverlauf}

Die mediane Zeit von arterieller Punktion zu Beginn der Intervention bis zur erfolgreichen Rekanalisation lag bei 50 Minuten, mit einem Mittelwert von 62 Minuten und einer Standardabweichung von 47 Minuten (IQR 31 - 86). Kein deutlicher Unterschied ergab sich in der Rekanalisationszeit von Verschlüssen in der vorderen bzw. hinteren Zirkulation (53 vs. 41 Minuten).

Durchschnittlich vergingen $344 \pm 237$ Minuten von Symptombeginn bis zur erfolgreichen mechanischen Rekanalisation, wobei der Median bei 295 Minuten lag (IQR 245 - 364).

\subsection{Rehabilitation und klinisches Behandlungsergebnis}

\subsubsection{Rehabilitationsverläufe}

Die behandelten Patienten verbrachten durchschnittlich $18 \pm 12$ Tage im Akutkrankenhaus, der Median lag bei 16 Tagen (IQR 10 - 23).

80 der fortlaufend beobachteten Patienten wurden anschließend in eine weiterbehandelnde Rehablilitationseinrichtung verlegt, wo sie durchschnittlich weitere $57 \pm 40$ Tage therapiert wurden (Median 49 Tage, IQR 29 - 66).

Entsprechend ergibt sich eine durchschnittliche Therapiezeit im Anschluss an den stattgefundenen Schlaganfall von $72 \pm 50$ Tagen (Median 67 Tage, IQR 38-96).

Patienten mit Gefäßverschlüssen in der vorderen Zirkulation hatten im Median eine um fast zwei Wochen kürzere Therapiedauer (64 Tage vs. 77 Tage), vorwiegend zurückzuführen auf eine längere mediane Rehabilitationsdauer (49 Tage vs. 55 Tage).

\subsubsection{Klinische Ergebnisse}

\subsubsection{Klinisches Behandlungsergebnis nach Klinikentlassung}

Der mittlere NIHSS-Wert verbesserte sich von Klinik-Aufnahme bis zum Zeitpunkt der Klinik-Entlassung von 17 Punkten auf 9,9 Punkte (SD $\pm 6,2, P<0.001$ ) (siehe Abbildung 28, Seite 74). Daraus ergibt sich eine durchschnittliche Verbesserung des NIHSS-Wertes von 7,1 Punkten. Der Median lag zu diesem Zeitpunkt bei 9 Punkten, gegenüber 18 Punkten bei Therapiebeginn. 
Bis zur Entlassung aus dem Krankenhaus erzielten 44 Patienten (48,4\%) eine klinische Verbesserung im NIHSS-Wert von $\geq 4$ Punkten, bei 21 Patienten $(23,1 \%)$ zeigte sich sogar eine Punktdifferenz von $\geq 10$ Punkten gegenüber dem Aufnahmewert.

Während bei Klinikaufnahme nach Symptombeginn der mediane mRS bei 5 (IQR 45) lag, konnte bei Entlassung aus dem Akutkrankenhaus ein medianer mRS von 4 festgestellt werden (IQR 4-5, $P=0.041)$. Der Mittelwert verbesserte sich dabei von 4,48 Punkten auf 4,12 Punkte ( $\varnothing-0,36$ Punkte).

Bei Klinikentlassung hatten 15 Patienten (16,5\%) ein gutes klinisches Endergebnis entsprechend einem $\mathrm{mRS} \leq 2$, wohingegen 36 Patienten $(39,6 \%)$ ein schlechtes Outcome $(m R S \geq 5)$ zeigten.

\subsubsection{Klinisches Behandlungsergebnis nach Rehabilitation}

Im Verlauf der Rehabilitation ging der mittlere NIHSS-Wert in der beobachteten Patientenpopulation von 9,9 Punkten auf 6,3 Punkte (SD $\pm 5,6 ; P<0.001)$ zurück ( $\varnothing-3,6$ Punkte). Der Median lag am Ende des Beobachtungszeitraumes bei einem NIHSS-Wert von 5, vormals 18 bzw. 5 Punkten bei Klinik-Aufnahme bzw. -Entlassung - dies entspricht einer medianen Reduzierung um 13 Punkte im Gesamtzeitraum.

Im Follow-up stieg die Zahl der Patienten mit einer NIHSS-Verbesserung um $\geq 4$ Punkte auf 52 (57\%), insgesamt 33 Patienten (36 \%) hatten eine Verbesserung um $\geq 10$ Punkte auf der NIHSS-Skala.

Insgesamt 23 Patienten (28,8 \%) hatten nach 90 Tagen bzw. nach Entlassung aus der Rehabilitation ein gutes klinisches Endergebnis, definiert als ein mRS von $\leq 2$ (siehe Abbildung 29, Seite 74). Andererseits wurde bei 28 Patienten (35,0 \%) die am Ende des Beobachtungszeitraumes bestehende Funktionseinschränkung als schlechtes klinisches Ergebnis gewertet ( $m R S \geq 5$ ). Im Follow-up betrug der mediane mRS 4 (IQR 2 - 6, P=0.001). 


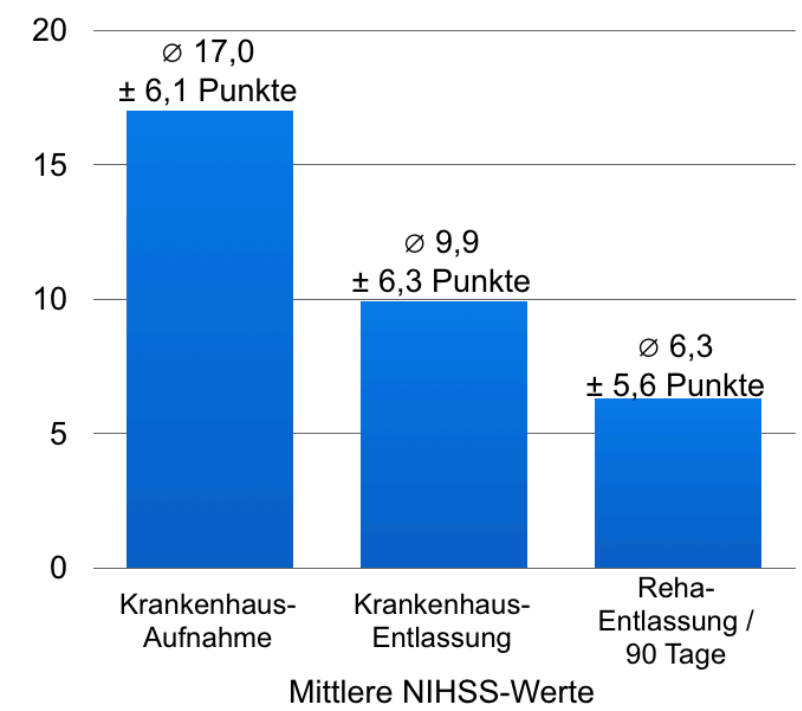

Abbildung 28 (links) - Darstellung

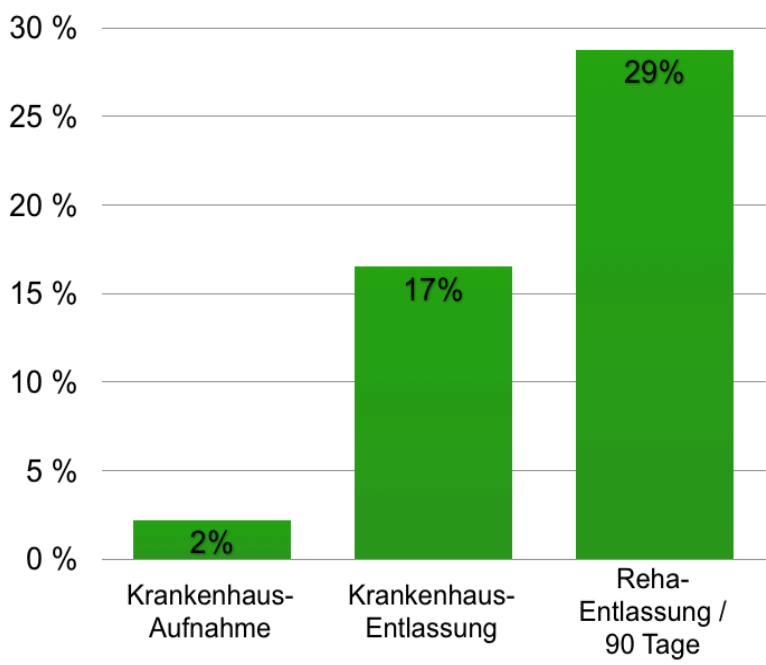

Prozentzahl der Patienten mit $\mathrm{mRS} \leq 2$

Beobachtungszeitraum.

Abbildung 29 (rechts) - Darstellung der Prozentzahl an Patienten mit einem mRS $\leq 2$ während des Beobachtungszeitraumes.

\subsubsection{Behandlungsergebnisse nach Verschlusslokalisation}

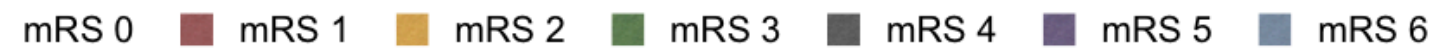

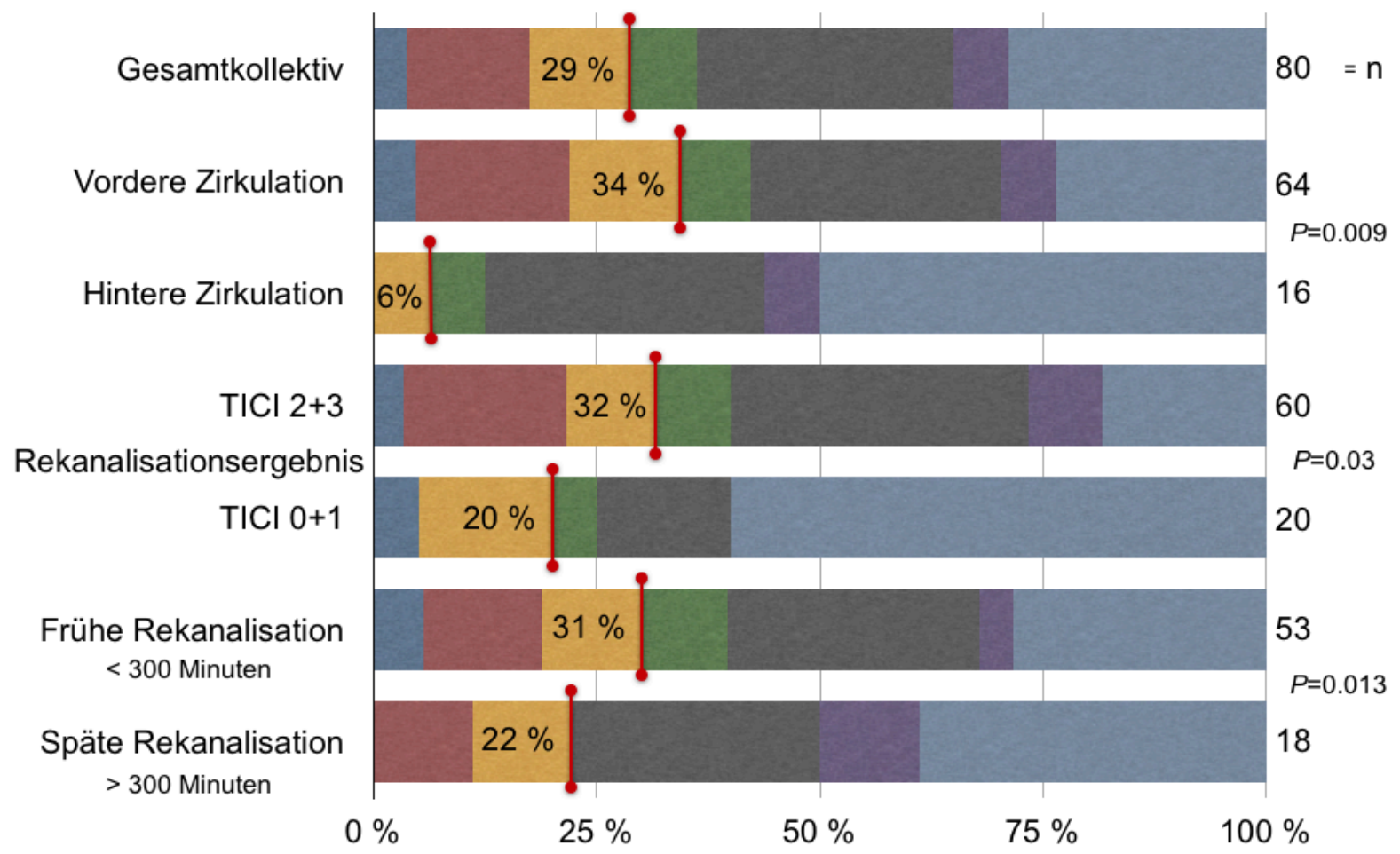

Abbildung 30 - Darstellung des klinischen Behandlungsergebnisses im Gesamtkollektiv und in den Subgruppen. Die Prozentzahl an Patienten mit einem guten klinischen Endergebnis $(m R S \leq 2)$ am Ende des Beobachtungszeitraumes ist angegeben. 
Betrachtet man die erzielten neurologisch-klinischen Endergebnisse bezüglich der festgestellten Verschlusslokalisationen ergeben sich folgende Ergebnisse:

Insgesamt erreichten Patienten mit Gefäßverschlüssen in der vorderen Zirkulation zu $34 \%$ ein gutes klinisches Endergebnis, wohingegen nur $6 \%$ der Patienten mit Verschlüssen der hinteren Zirkulation ein gutes Outcome nach Rehabilitation hatten $(P=0.009)$ (siehe Abbildung 30, Seite 74).

Bei differenzierter Betrachtung der Verschlussorte konnte ein gutes klinisches Endergebnis (mRS $\leq 2$ ) bei 10 Patienten (29\%) mit MCA-Verschlüssen, bei 9 Patienten (50 \%) mit Carotis-T-Okklusionen, in 3 Fällen (30 \%) mit ICA- und in einem Fall $(100 \%)$ mit PCA-Verschluss erreicht werden $(P=0.10)$. Im Gegensatz dazu wurde ein schlechtes Endergebnis (mRS $\geq 5$ ) in einem Fall $(100 \%)$ mit CCAVerschluss, bei 8 Patienten (67 \%) mit BA-Okklusion und bei 2 Patienten (67\%) mit kombinierten BA- und VA-Verschlüssen diagnostiziert.

\subsubsection{Rehabilitationsergebnisse nach Rekanalisationsergebnis}

Folgende Ergebnisse ergeben sich aus dem Vergleich der Rehabilitationsergebnisse in Abhängigkeit vom interventionell erzielten Gefäßresultat: In der Kohorte der erfolgreich rekanalisierten Patienten (TICl 2 oder 3 ) hatten $32 \%$ (19/60) einen mRS $\leq 2$ (gutes klinisches Ergebnis) und $27 \%$ (16/60) einen mRS $\geq 5$ (schlechtes klinisches Ergebnis) nach der Rehabilitation. Die Patienten hatten eine mediane NIHSS-Verbesserung im Follow-up von 6 Punkten, die Mortalität in dieser Subgruppe lag bei $18,3 \%(11 / 60)$.

Im Gegensatz dazu hatten Patienten, die nicht erfolgreich rekanalisiert werden konnten ( $\mathrm{TICl} 0$ oder 1$)$ in $20 \%$ (4/20) ein gutes Outcome und in $60 \%$ (12/20) ein schlechtes Endergebnis $(P=0.03)$. In dieser Gruppe lag die mediane NIHSSVerbesserung bei 8 Punkten und die Mortalität betrug im Beobachtungszeitraum $60 \%(12 / 20)$.

Siehe Abbildung 30 (Seite 74) für eine graphische Darstellung der Ergebnisse.

\subsubsection{Rehabilitationsergebnisse nach früher bzw. später Rekanalisation}

Im Vergleich der Rehabilitationsergebnisse nach früher $(\leq 300$ Minuten nach Symptombeginn) oder später erfolgreicher Rekanalisation (> 300 Minuten nach Symptombeginn) ergeben sich ebenfalls deutliche Unterschiede (siehe Abbildungen 30 und 31 , Seiten 74 und 76 ). 
In der Patienten-Subgruppe, bei der die Rekanalisation frühzeitig erfolgte, hatten $41 \%$ der Patienten (13/32) ein gutes Outcome und in 16\% der Fälle (5/32) ein schlechtes Endergebnis.

Eine späte erfolgreiche Rekanalisation führte in 19\% (6/32) zu einem guten (mRS $\leq 2)$ und in $53 \%(17 / 32)$ zu einem schlechten klinischen Endergebnis ( $m R S \geq 5$ ) $(P=0.013)$. In der Subgruppe der spät rekanalisierten Patienten lag die Mortalität 10 \% höher, als bei der früh rekanalisierten Patientengruppe (38,9 \% vs. 28,9 \%).

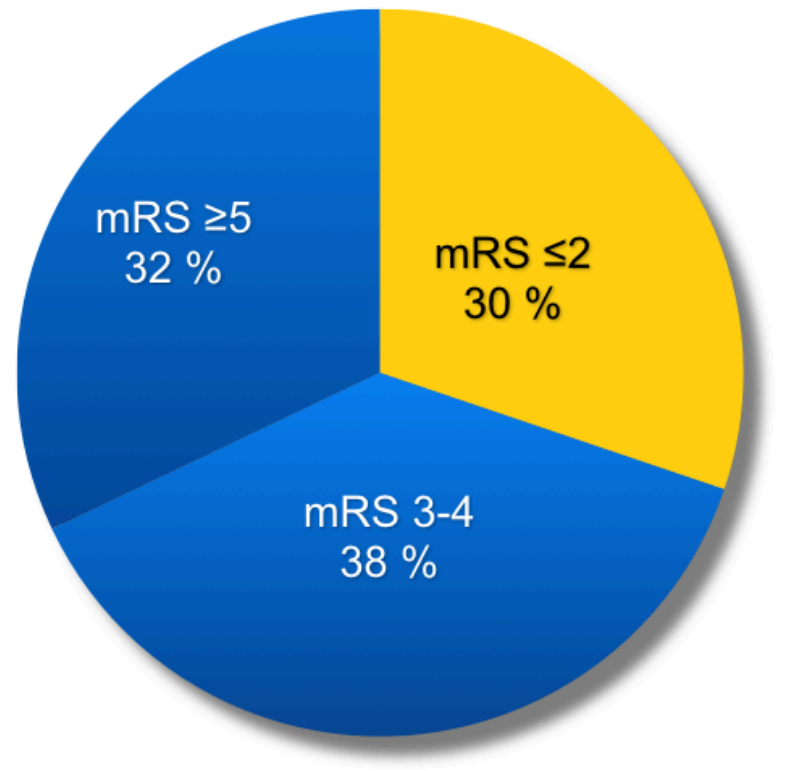

Frühe Rekanalisation

$<300$ min

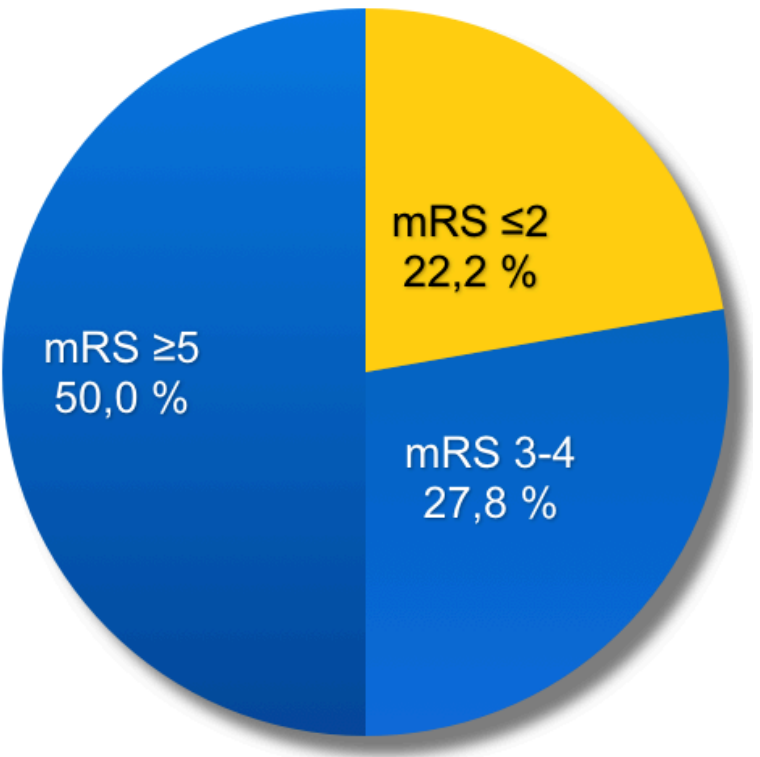

Späte Rekanalisation $>300 \mathrm{~min}$

Abbildung 31 - Darstellung des klinischen Behandlungsergebnisses der rekanalisierten Patienten. Eine frühe Rekanalisation (<300 Minuten nach Symptombeginn) führte öfter zu einem guten klinischen Endergebnis ( $m R S \leq 2)$ als eine spätere Rekanalisation.

\subsubsection{Mortalität}

Die Gesamtmortalität im Beobachtungszeitraum betrug 25,3 \% (23/91). Davon verstarben 20 Patienten (22,0 \%) während des Klinikaufenthaltes und weitere 3,3 \% (3 Patienten) während der Rehabilitation (siehe Abbildung 30, Seite 74).

Die Patienten im beschriebenen Kollektiv waren durchschnittlich 70,0 Jahre alt zum Todeszeitpunkt, der im Durchschnitt 6,5 Tage nach dem Schlaganfall lag. Die verstorbenen Patienten hatten im Mittel einen initialen NIHSS-Aufnahmewert von 18,2 Punkten, also über dem Wert der Gesamtpopulation ( $\varnothing 17$ NIHSS-Punkte).

Gefäßverschlüsse der vorderen Zirkulation waren mit einer deutlich geringeren Mortalität assoziiert (23,4 \%) als Okklusionen der hinteren Zirkulation (50 \%). 


\subsection{Fallbeispiele}

Im Folgenden sollen zwei Patientenbeispiele exemplarisch dargestellt werden.

\subsubsection{Fallbeispiel 1}

Eine 25-jährige Patientin wurde mit progredienter Vigilanzminderung, Minderbewegung der rechten Körperseite sowie positivem Pyramidenbahnzeichen rechts aus einem auswärtigen Krankenhaus wegen des Verdachts auf eine links hemisphärische Ischämie aufgenommen (NIHSS 14, mRS 5). In der multimodalen MRT-Bildgebung zeigte sich ein Verschluss der A. cerebri media links (Abbildung 32 A), eine deutliche Perfusionsverzögerung im dazugehörigen Territorium, sowie eine noch relativ kleine Diffusionseinschränkung (Abbildungen 32 B und C). Nach Ausschluss einer intrakraniellen Blutung erfolgte die Indikationsstellung für eine mechanische Lysetherapie. 4,5 Stunden nach Symptombeginn wurde mit der Angiographie begonnen, über einen 6F-Envoy-Katheter erfolgte die Darstellung der intrakraniellen Gefäße. Es bestätigte sich ein vollständiger Media-Verschluss im M1Segment, entsprechend $\mathrm{TICl} 0$ (Abbildung $32 \mathrm{D}$ ). Nach der Plazierung des Penumbra-Reperfusionskatheters 041 im Bereich proximal des Thrombus, erfolgte eine zügige und problemlose Rekanalisation über einen Zeitraum von 24 Minuten.

Nach Anfertigung von Kontrollserien zeigte sich eine komplette Rekanalisation des Media-Hauptstammes ( $\mathrm{TICl} 3$ ) (Abbildung $32 \mathrm{E}$ ). Anschließend erfolgte eine langsame, intraarterielle Applikation von $20 \mathrm{mg}$ rt-PA lokal durch den liegenden Führungskatheter für die Lyse kleiner distaler Emboli im Media-Stromgebiet.

Einen Tag nach mechanischer Thrombektomie ist im Kontroll-CCT eine umschriebene Infarzierung der Basalganglien links mit minimaler Hämorrhagisierung zu erkennen (Abbildung 32 F). Das Infarktareal geht nicht über die präinterventionell bereits erkennbare Diffusionseinschränkung hinaus.

Nach 18-tägigem Aufenthalt im Akutkrankenhaus konnte die Patientin in deutlich gebessertem klinisch-neurologischen Zustand (NIHSS: 5, mRS 2) nach Teilnahme am Frührehabilitationsprogramm in die Rehabilitation entlassen werden. Die bei Entlassung noch vorhandenen leichtgradigen Koordinations- und Wortfindungsstörungen konnten erfolgreich in der folgenden 22-tägigen Rehabilitationsphase therapiert werden. Abschließend konnte die Patientin ohne signifikante Einschränkungen entlassen werden und ist in der Lage ein selbstständiges Leben zu führen (NIHSS 0, mRS 1). 


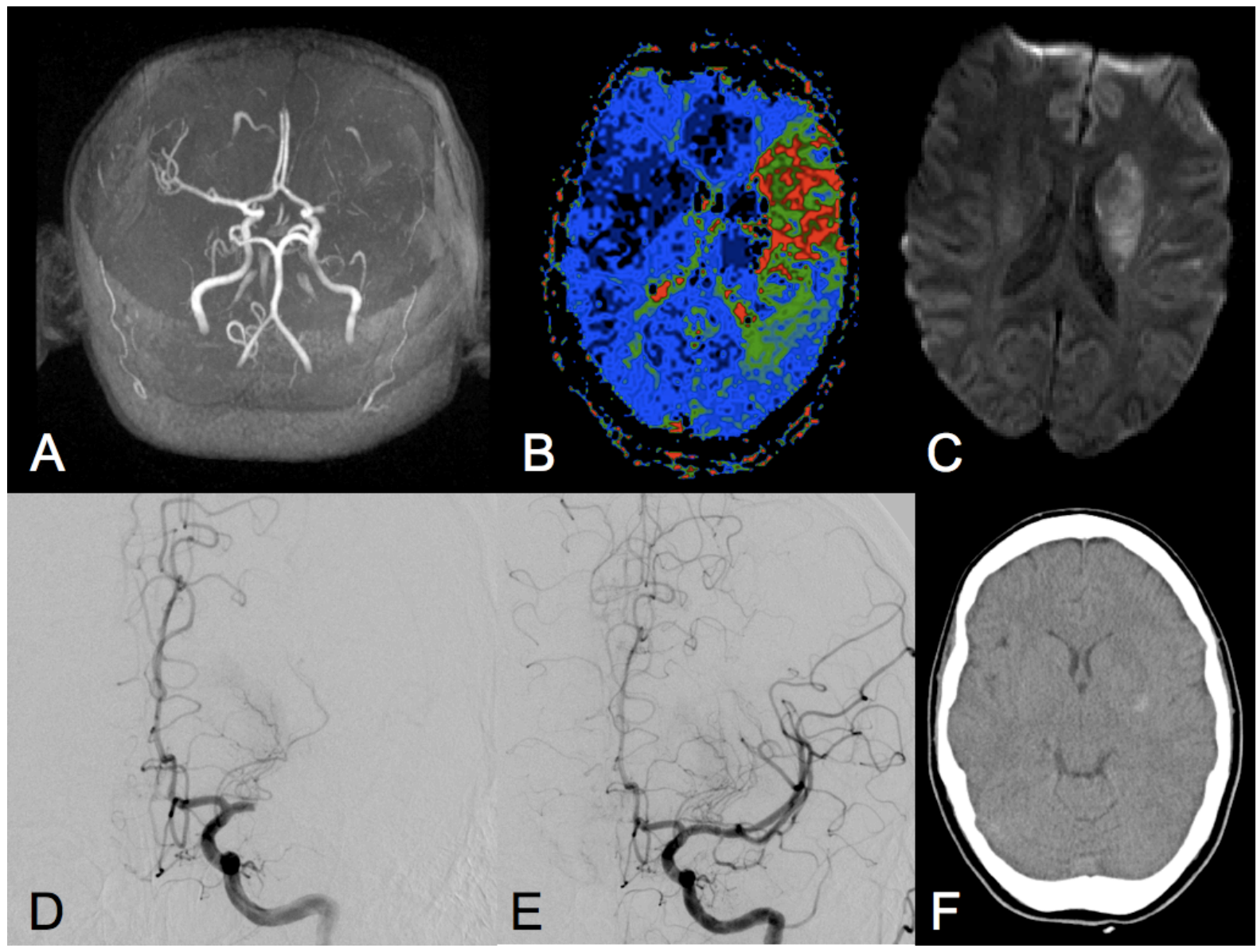

Abbildung 32 - Fallbeispiel 1 der Aspirationstherapie mittels Penumbra System

A) In der initialen Magnetresonanzangiographie zeigt sich ein kompletter Verschluss der A. cerebri media links.

B) Im linksseitigen Media-Territorium wird in der errechneten TTP-Map (time to peak) eine ausgedehnte Perfusionsverzögerung sichtbar.

C) Die diffusionsgewichtete Bildgebung (DWI, $b=1000)$ stellt den hyperintensen Infarktkern dar. Dieser ist im Vergleich zum Mediaterritorium und der Ausdehnung der Perfusionsverzögerung klein.

D) In der DSA bestätigt sich der vollständige Media-Verschluss links im M1-Segment (TICI 0).

E) DSA nach erfolgreicher Rekanalisierung des M1-Segmentes (TICI 3).

F) Im Verlaufs-CT nach einem Tag ist eine sehr kleine Hämorrhagisierung des Infarktgebietes im Bereich der Basalganglien links zu erkennen. Das Infarktareal geht nicht über die präinterventionell bereits vorhandene Diffusionseinschränkung hinaus. 


\subsubsection{Fallbeispiel 2}

Der zweite Patient ist ein 50-jähriger Mann, der mit akut aufgetretener brachiofazial betonter Hemiparese rechts und globaler Aphasie aufgefunden wurde. Der NIHSS betrug bei Aufnahme 12 Punkte, bei einem mRS von 5. Folgende kardiovaskuläre Risikofaktoren lagen vor: Arterieller Hypertonus, entgleister insulinpflichtiger Diabetes mellitus und Nikotinabusus.

Anhand der initialen CT-Angiographie- und CT-Perfusionsbildgebung konnte ein Verschluss der ACI und MCA links nachweisen werden, mit deutlicher Verminderung des zerebralen Blutflusses links fronto-operkulär, insulär und am rechten Putamen (Abbildungen $33 \mathrm{~A}$ und $\mathrm{B}$ ).

Direkt nach der CT-Bildgebung wurde eine intravenöse Bridging-Lysetherapie mit insgesamt 40 mg rt-PA gestartet, davon $10 \mathrm{mg}$ rt-PA als Bolus.

249 Minuten nach Symptombeginn konnte der Patient in die Angiographie gebracht werden und in den angiographischen Serien zeigten sich eine filiforme Abgangsstenose der linken Carotis interna sowie eine vollständige Thrombose auf Höhe des Siphons ( $\mathrm{TICl}$ 0). Mittig im Bereich der Stenose wurde ein Carotid Wall Stent (7 $\mathrm{mm} \mathrm{x}$ $40 \mathrm{~mm}$, Boston Scientific, Natick, MA, USA) freigesetzt und mit einem SterlingBallonkatheter $(5 \mathrm{~mm}$ × $20 \mathrm{~mm}$ ) nachdilatiert. Anschließend zeigte sich eine sehr gute Rekonstruktion des Gefäßlumens. Die Abbildungen 33 C und D zeigen den distalen thrombotischen Verschluss des Carotissiphons.

Daraufhin Vorführen eines Penumbra-Reperfusionskatheters 054 und -Separators bis zum linken Karotissiphon und Beginn der mechanischen Thrombektomie. Nach mehreren Versuchen gelang eine Entfernung des Thrombus bis zum Carotis-T.

Eine Plazierung des Penumbra Systems (054, 041, 032) distal des Abganges der A. ophthalmica links in der $A$. cerebri media gelang in mehreren Versuchen nicht. Periprozedural erfolgte dann die Gabe von insgesamt 15 mg rt-PA intraarteriell.

Im letzten Versuch gelang ein coaxiales Vorgehen mit Vorführen des Reperfusionskatheters 054 über den Reperfusionskatheter 032 und über einen in die linke MCA platzierten Synchro-Mikrodraht. Nach mehreren Reperfusionsmanövern zeigen die angiographischen Serien schließlich eine erfolgreiche Rekanalisation des M1-Segmentes links und auch der prominenten M2-Äste links. Die Abschlussserien (Abbildungen $33 \mathbf{E}$ und F) wiesen ein sehr gutes Ergebnis mit Rekanalisation der proximalen Media-Äste und gute Perfusionsverhältnisse im größten Anteil des Mediastromgebietes links auf $(\mathrm{TICl} 3)$. Nach einer Angiographiedauer von 75 
Minuten verblieb lediglich in der peripheren linken A. cerebri anterior eine Flussverzögerung. Im postinterventionell nach 24 Stunden durchgeführten CCT wurde eine Einblutung im basalen Temporallappen und im Infarktgebiet festgestellt, die jedoch keine weitere invasive Therapie erforderte.

Nach dem 34-tägigen Aufenthalt im Akutkrankenhaus konnte der Patient in deutlich gebessertem klinisch-neurologischen Zustand (NIHSS: 5, mRS: 4) in die Rehabilitation entlassen werden. Nach Abschluss der Reha-Maßnahmen persistierte der NIHSS bei 5 Punkten, der mRS verbesserte sich weiter auf 2 Punkte. Der Patient konnte ein selbstständiges Leben führen und benötigte im Alltag weiterhin keine dauerhafte Unterstützung.

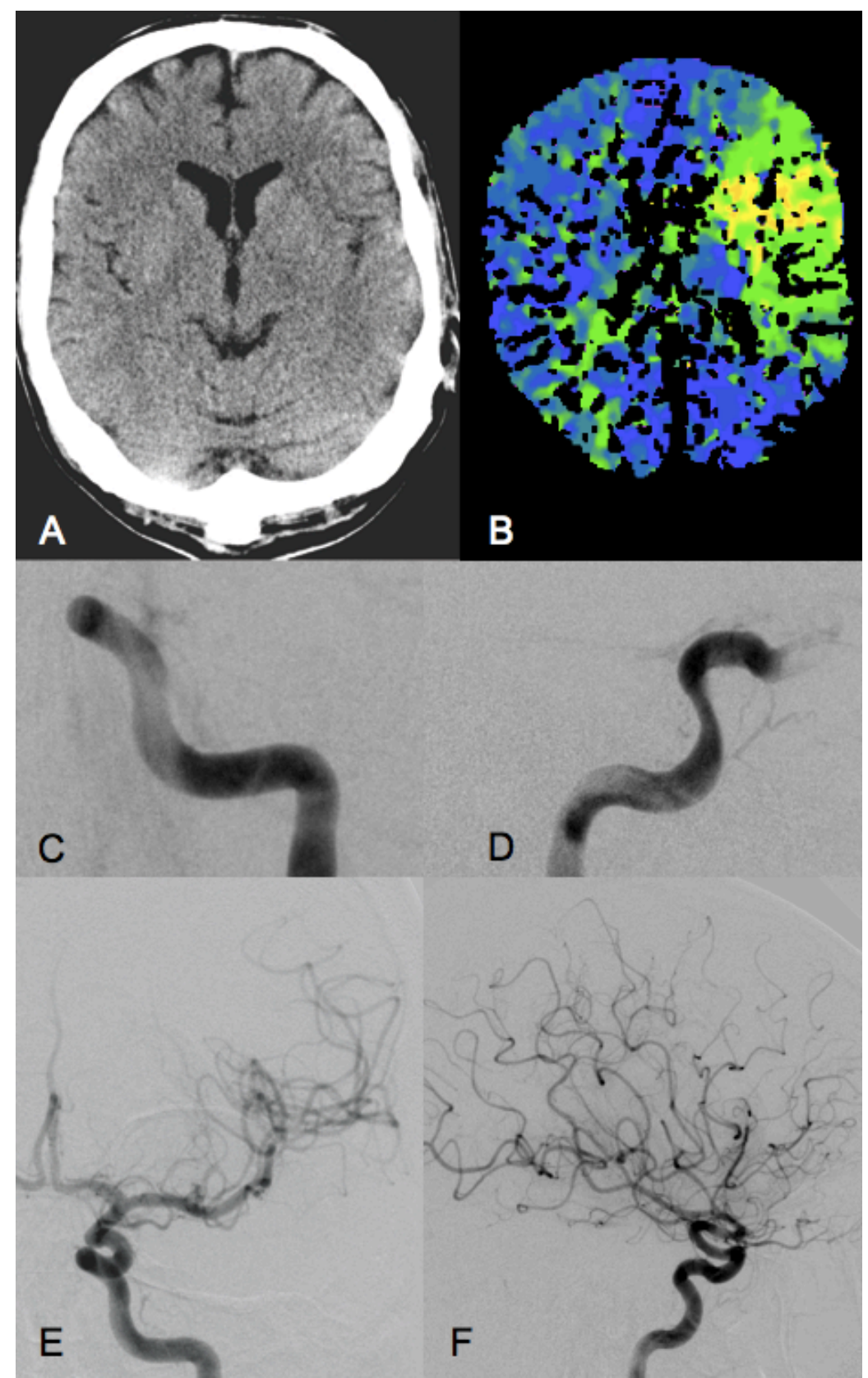

Abbildung 33 - Fallbeispiel 2 der Aspirationstherapie mittels Penumbra System 
A) Initiale native Computertomographie zum Ausschluss einer intrakraniellen Blutung.

B) Anhand der errechneten TTP-Map (time to peak) ist eine deutliche Perfusionsverzögerung links fronto-operkulär, insulär und am rechten Putamen erkennbar.

C) Die erste anterior-posteriore DSA-Serie bestätigt einen vollständigen Verschluss des Karotissiphons ( $\mathrm{TICl} 0$ ).

D) Besonders in der lateralen Projektion lässt sich der langstreckige Thrombus sehr gut erkennen.

E) Nach Stenting der ACl-Abgangsstenose konnte der thrombotische Verschluss durch mechanische Aspirationsthrombektomie mit dem Penumbra System vollständig rekanalisiert werden ( $\mathrm{TICl} 3$ ). Sowohl die $\mathrm{ACl}$, als auch die proximalen MCA-Äste und die ACA zeigen sich komplett reperfundiert.

F) Die laterale Projektion zeigt das angiographische Endergebnis nach mechanischer Thrombektomie. 


\section{Diskussion}

Die Therapie des akuten ischämischen Schlaganfalls hat sich in den letzten zwei Jahrzehnten grundlegend verändert. Die Einführung von Stroke-Units und der systemischen Fibrinolyse mit rt-PA hat zu einer signifikanten Reduktion der Todesfälle und schwerwiegender körperlicher Beeinträchtigungen geführt.

Trotzdem ist der Schlaganfall noch immer der Hauptgrund für Behinderungen bei Erwachsenen in den USA und Europa und die zweitwichtigste Todesursache weltweit (Feigin et al. 2009; WHO 2008). Weiterhin muss aufgrund der Altersverteilung zerebrovaskulärer Erkrankungen durch das steigende Durchschnittsalter der deutschen Bevölkerung mit einer deutlichen Zunahme von Schlaganfällen gerechnet werden.

Die intravenöse rt-PA-Applikation ist bis heute die nützlichste, wissenschaftlich belegte Therapieform für den akuten ischämischen Schlaganfall, wobei die Anwendung nur bis zu 3 Stunden nach Symptombeginn zugelassen ist, ein gradueller Wirksamkeitsverlust über die Zeit auftritt und bei langstreckigen Gefäßverschlüssen über $8 \mathrm{~mm}$ Länge die Erfolgschance gering ist (Hacke et al. 2008; Riedel et al. 2011). Die schnelle, erfolgreiche Reperfusion ischämischen Hirngewebes ist, neben anderen Faktoren, ein Hauptfaktor für ein gutes klinisches Behandlungsergebnis von Schlaganfallpatienten (Rha und Saver 2007).

Die genannten Limitationen führen dazu, dass heute in Deutschland nur bis zu 14 \% der Schlaganfallpatienten mit den, entsprechend den Leitlinien, verfügbaren fibrinolytischen Therapien behandelt werden können (Grau et al. 2010).

\subsection{Ergebnisdiskussion}

Die Diskussion der Ergebnisse orientiert sich auch im Hinblick auf die Zielsetzungen dieser Arbeit (siehe Kapitel 1.5, Seite 43) primär an den untersuchten Parametern, um leichter und übersichtlicher einen umfassenden Vergleich mit bisherigen Studien ziehen zu können.

\subsubsection{Rekanalisationsergebnisse}

In dieser Studie wurden retrospektiv die angiographischen Bildserien und klinischen Verläufe von 91 Patienten mit akutem ischämischen Schlaganfall ausgewertet. Die postinterventionellen Aufnahmen zeigten in 76,9 \% der Fälle eine erfolgreiche Gefäßrekanalisation, gemäß $\mathrm{TICl} 2$ oder 3. Vergleichbare Rekanalisationsraten 
zwischen 67 - 100 \% zum Penumbra System, in Kombination mit intravenöser und/oder intraarterieller Fibrinolyse, sind in vorwiegend kleineren Fallsammlungen ( 330 Patienten) publiziert (Kreusch und Knauth 2011). Diese Arbeit bestätigt somit die hohe technische Rekanalisationsrate der Aspirationsthrombektomie, die mit dem Penumbra System erreicht werden kann.

Das Ergebnis dieser Arbeit ist vergleichbar mit der beschriebenen Rekanalisationsrate im Penumbra Pivotal Stroke Trial (81,6 \%, TIMI Score 2 oder 3) und im POST Trial (87 \%, TIMI Score 2 oder 3) (Penumbra Pivotal Stroke Trial Investigators 2009; Tarr et al. 2010). Die Rekanalisationsrate von $76,9 \%$ ist höher als die beschriebenen Erfolgsraten in der PROACT-II-Studie (66\%, intraarterielle rekombinante Pro-Urokinase, TIMI Score), im Multi MERCI Trial (69.5\%, kombinierte intraarterielle rt-PA-Gabe und mechanische Thrombektomie, TIMI Score) oder der IMS-II-Studie (73 \%, intraarterielle Sonothrombolyse, TIMI Score) (Furlan et al. 1999; IMS II Trial Investigators 2007; Smith et al. 2008). In einer weiteren Studie von Mattle et al. über 55 Patienten mit MCA-Hauptstamm-Verschluss und intraarterieller Lysetherapie (rt-PA) wurde eine Rekanalisationsrate von 71 \% (TIMI Score 2 oder 3) berichtet (Mattle et al. 2008).

Ein weiteres interessantes Resultat ist die niedrigere Rekanalisationsrate von $60 \%$ bei Patienten, denen vor Katheter-basierter mechanischer Intervention bereits die gesamte Dosis rt-PA intravenös appliziert wurde. Die zusätzliche intraarterielle Gabe von rt-PA kann in diesen Fällen in Erwägung gezogen werden, seitdem Shaltoni et al. gezeigt haben, dass die intraarterielle rt-PA-Gabe nach Verabreichung der gesamten intravenösen rt-PA-Dosis sicher zu sein scheint und dadurch eine höhere Rekanalisationsrate und ein besseres Outcome erreicht werden können (Shaltoni et al. 2007). In der Mehrzahl der Studien kommt die mechanische Rekanalisation als Ergänzung zur medikamentösen Fibrinolyse zum Einsatz.

Castano et al. berichten eine zu $90 \%$ erfolgreiche Rekanalisation ( $\mathrm{TICl} 2 \mathrm{~b}$ oder 3 ) mit einem neuen Stentriever für die Flusswiederherstellung, jedoch bemerken die Autoren die Bedeutung der Thrombuslänge als wichtigen Faktor (Castano et al. 2010). Sie waren dazu gezwungen bei 4 Patienten vor der Flusswiederherstellung mittels Stent eine Thrombus-Extraktion durchzuführen, da die Thromben die Stentlänge überschritten. Die mediane Zeitspanne von arterieller Punktion bis zur erfolgreichen Rekanalisation betrug in dieser letzten Studie 70 Minuten, dies ist deutlich länger, als in dieser Arbeit beschrieben (Median: 49 Minuten). 
Der Unterschied zu einigen dieser Studien besteht in der Lokalisation des Gefäßverschlusses. Das in dieser Arbeit beschriebene Patientenkollektiv hatte zu $44 \%$ MCA- und PCA-Verschlüsse, die weiteren Verschlusslokalisationen von $56 \%$ waren in Gefäßen mit größerem Durchmesser und dementsprechend mehr Thrombusmaterial zu finden (proximale oder distale ICA- und BA-Okklusionen).

Die Ergebnisse dieser Arbeit sind auch bedeutsam für Patienten, welche nicht die Einschlusskriterien für eine intravenöse Lysetherapie erfüllen. Bei 14 dokumentierten Fällen ohne medikamentöse Lyse konnten eine erfolgreiche Rekanalisationsrate von $79 \%$ gezeigt werden, allein durch die mechanische Rekanalisation. Patienten nach großen chirurgischen Eingriffen oder Trauma, mit einer verlangsamten Blutgerinnungszeit (INR > 1,7, Quick < 50 \%), während Schwangerschaft oder mit einer Klinikaufnahme außerhalb des Zeitfensters von 4,5 Stunden für eine medikamentöse Lysetherapie haben immer noch eine Chance auf eine erfolgreiche Rekanalisation und ein verbessertes klinisches Behandlungsergebnis bei Einsatz des Penumbra Systems.

\subsubsection{Rehabilitationsergebnisse}

Bezüglich des klinischen Endergebnisses können ebenfalls ähnliche Resultate, wie im Penumbra Pivotal Trial, beobachtet werden: 23 \% der Patienten zeigten eine NIHSS-Verbesserung von $\geq 10$ Punkten bei Krankenhausentlassung und $29 \%$ hatten in der Verlaufskontrolle einen $m R S \leq 2$, was für ein selbstständiges Alltagsleben steht. $32 \%$ der erfolgreich rekanalisierten Patienten hatten ein gutes klinisches Behandlungsergebnis, während nur $20 \%$ der nicht rekanalisierten Patienten bei Behandlungsende einen $\mathrm{mRS} \leq 2$ hatten. Während die absoluten Differenzen zwischen diesen beiden Gruppen nicht groß sind, so sind die relativen Differenzen sehr deutlich und statistisch signifikant $(P=0.03)$.

Die Gesamtmortalität von $25 \%$ ist um 7 - $10 \%$ niedriger als bei historischen Vergleichsgruppen (Penumbra Pivotal Stroke Trial Investigators 2009; Smith et al. 2008). In Korrelation zu den bisherigen Veröffentlichungen entspricht die aus dieser Arbeit resultierende Mortalitätsrate genau dem Mittelwert der Publikationen von 25,8 \% nach 90 Tagen (siehe Tabelle 4, Seite 36/37).

Der Prozentsatz von gutem klinischen Outcome ist in diesem Patientenkollektiv geringer als in einer zusammenfassenden Analyse von intravenösen rt-PA-Studien (49 \% mRS $\leq$ 2) (Hacke et al. 2004). Bei diesem Vergleich muss jedoch der Einfluss 
des NIHSS-Punktwertes bei initialer Klinikaufnahme auf das neurologische Endergebnis berücksichtigt werden. Während Patienten in dieser Pool-Analyse einen mittleren NIHSS von 11 aufwiesen, lag der Aufnahme-NIHSS in der eigenen Patientenkohorte durchschnittlich bei 17 . Wie in diesen Studien ebenfalls beschrieben, erreichen $44 \%$ der Patienten, welche mit einem initialen NIHSS von 11 aufgenommen werden, ein gutes klinisches Endergebnis ( $\mathrm{mRS} \leq 2)$ ohne irgendeine spezifische Therapie (Placebo-Gruppe). Bei einem mittleren NIHSS-Punktwert von 18 fällt diese Prozentzahl auf 10 - 15 \% (IMS Study Group 2004; NINDS t-PA Stroke Study Group 1997).

Ein weiterer wichtiger Faktor, welcher signifikant das Outcome in unserer Studie beeinflusst, ist die Zeitspanne vom Symptombeginn bis zur partiellen oder vollständigen Rekanalisation. Während Mattle et al. einen Mittelwert von 244 Minuten von Symptombeginn bis Therapiestart berichten, benötigten wir 294 Minuten (Median) um die verschlossenen Hirngefäße wiederzueröffnen. Dieser Aspekt, zusätzlich zur größeren Heterogenität der Verschlusslokalisationen in unserem Kollektiv, wie zuvor beschrieben, kann als Erklärungsansatz für die Differenzen in gutem klinischem Endergebnis und Mortalität zwischen unserem Kollektiv und dem von Mattle et al. dienen ( $29 \% \mathrm{mRS} \leq 2$ und $25 \%$ Mortalität; vs. $53 \% \mathrm{mRS} \leq 2$ und $7 \%$ Mortalität) (Mattle et al. 2008).

Die in dieser Arbeit beschriebenen finalen Behandlungsergebnisse bedürfen einer sorgfältigen Beurteilung, da es sich um eine retrospektive Studie handelt. Der erweiterte therapeutische Nutzen durch das Penumbra System für die Patienten im Vergleich zur medikamentösen Thrombolyse kann nur durch randomisierte, kontrollierte Studien wissenschaftlich belegt werden (Coutts und Goyal 2009; Saver 2011; Schellinger und Hacke 2010).

\subsubsection{Komplikationen}

Im Rahmen dieser Arbeit wurden wenige Komplikationen bei Einsatz des Penumbra Systems zur mechanischen Rekanalisation beobachtet. Kein beschriebener Todesfall war auf technische Fehler zurückzuführen.

Die beschriebenen Inzidenzen von $\mathrm{ICH}(20,9 \%)$ und $\mathrm{SICH}(5,5 \%)$ sind vergleichbar mit historischen Vergleichswerten von anderen Studien zur mechanischen Thrombektomie und zur medikamentösen Fibrinolyse. In der Multi-MERCI-Studie zur Evaluation des MERCI Retrievers wurden ICHs in 30,5 \% der Fälle beobachtet, SICH 
bei 9,8 \% der Patienten. Interessanterweise wurde kein statistischer Unterschied im Auftreten von SICH bei Thrombektomie \pm rt-PA gefunden (Smith et al. 2008). In der PROACT-II-Studie traten bei intraarterieller Prourokinase-Applikation sogar in $35 \%$ der Fälle ICHs nach 24 Stunden und bei 68 \% der Patienten innerhalb von 10 Tagen auf (Furlan et al. 1999). In vergleichbaren Fallpublikationen zum Penumbra System (siehe Tabelle 4, Seite 36/37) wurden SICH-Raten von 0 - 11,2 \% beschrieben, womit das Ergebnis dieser Arbeit die relativ niedrige Häufigkeit dieser Komplikation bestätigt.

Die Mehrzahl an postprozedural auftretenden Hirnblutungen sind hämorrhagische Transformationen von ischämischen Infarkten, ein sehr häufig beobachtetes Phänomen nach Gefäßwiedereröffnung. Es wurde vielfach vermutet, dass die Gefäßrekanalisation einen negativen Einfluss auf die hämorrhagische Transformation des Infarktes haben könnte. Diese Annahme wurde durch die Metastudie von Rha und Saver widerlegt, zwischen rekanalisierten und nicht-rekanalisierten Patienten ergab sich kein signifikanter Unterschied in Bezug auf symptomatische intrakranielle Hämmorrhagien (13,7 \% vs. 12,5 \%) (Rha und Saver 2007). Einhergehend mit dieser Publikation konnte in dieser Arbeit ebenfalls kein statistisch signifikanter Zusammenhang zwischen dem Auftreten von $\mathrm{ICH}$ und dem Rekanalisationsergebnis gefunden werden $(P=0.38)$. Bei Einsatz der medikamentösen IVT waren in der ECASS-III-Studie die Inzidenzen für $\mathrm{ICH}$ und $\mathrm{SICH} 27,0 \%$ bzw. 2,4 \%, wobei Hämorrhagien statistisch signifikant mit dem Einsatz von rt-PA korrelierten (Hacke et al. 2008). Bei Verwendung des Penumbra Systems mit mechanischer \pm medikamentöser Lyse konnte dieser signifikante Zusammen-hang nicht identifiziert werden $(P=0.58)$. Ein Erklärungsansatz ist der geringere rt-PA-Gebrauch in dieser Arbeit (Median $40 \mathrm{mg} /$ Patient). In vielen Fällen wurde auf die maximale rt-PA-Dosis verzichtet und nur eine Bridging-Lyse verwendet; die ECASS-III-Studie setzte ein oberes Dosislimit von 90 mg bei der üblichen Dosierung von 0,9 mg rt-PA/kg.

Vasospasmen wurden in dieser Arbeit bei 7,7 \% der Interventionen beobachtet. In einer neuen Arbeit zum Solitaire FR-Stentriever trat diese intraprozedurale Komplikation in $32 \%$ der Fälle wesentlich häufiger auf, konnte jedoch zuverlässig durch intraarterielle Nimodipin-Gabe therapiert werden (Machi et al. 2011). Auch für den MERCI Retriever ist diese Nebenwirkung beschrieben (Gupta 2009). 
Technische Fehlerraten des Penumbra Systems sind sehr selten. In dieser Arbeit sind zwei Separator-Brüche (siehe Kapitel 3.2.4.1, Seite 67) aus einem Zeitraum von 3 Jahren beschrieben, wobei in beiden Zwischenfällen kein Fremdmaterial im Patienten zurückblieb. Kulcsár et al. publizierten ebenfalls einen Fall eines aufgetretenen distales Separatorbruchs, wo das Fragment jedoch im Thrombus im M2-Segment verblieb (Kulcsar et al. 2010 a). Der mittlerweile von Penumbra Inc. (Alameda, CA, USA) überarbeitete Separator FLEX verspricht eine weichere Spitze und einen höheren Ermüdungswiderstand, sodass Brüche und der häufige intraprozedurale Austausch von Separatoren abnehmen wird. In anderen Studien, wie beispielsweise dem MERCI Trial, ist eine ähnliche Bruchrate von 3,2 \% berichtet, 2 Todesfälle (1,4 \%) wurden auf Materialbruch zurückgeführt (Smith et al. 2005).

Trotz der hohen beschriebenen Rekanalisationsraten durch das Penumbra System ist eine Rekanalisation nicht immer möglich, besonders dann nicht, wenn die Festigkeit des Thrombus kein Vorschieben des Separators ermöglicht oder der Verschluss sich in einem sehr stark gewundenen Gefäßsegment befindet. In diesen Fällen kann die kürzlich von Kang et al. beschriebene "modifizierte Penumbra System Technik" erfolgreich sein (Kang et al. 2011). Kennzeichnend für diese vereinfachte Modifikation des Penumbra Systems ist, dass lediglich der Reperfusionskatheter genutzt wird. Dieser wird mit seiner Spitze direkt in den proximalen Teil des Thrombus geführt und durch kräftigen manuellen Sog mit einer Spritze (20 oder $50 \mathrm{ml}$ ) dort verankert. Anschließend wird der Reperfusionskatheter mitsamt angesaugtem Thrombus entfernt. Nach der veröffentlichten Fallserie erlaubt diese modifizierte Technik eine sichere und effektive Rekanalisation (100\%). Dennoch muss hierbei bedacht werden, dass der verstärkte manuelle Sog das Risiko für Verletzungen des umgebenden Endotheliums erhöht, wenn die Katheterspitze direkten Kontakt mit der Gefäßwand bekommt.

Die in einer kleinen Fallsammlung (27 Patienten) berichtete Komplikation von distalen Embolien in 48,1 \% der Interventionen erscheint sehr hoch und wird in keiner anderen Publikation zum Penumbra System erwähnt (Menon et al. 2010). Die intraarterielle rt-PA-Gabe erzielt effektiv die Fibrinolyse kleiner Emboli, sofern diese im Rahmen einer Thrombektomie auftreten.

Zusammenfassend lässt sich feststellen, dass das Penumbra System einen sicheren, komplikationsarmen Einsatz bei Patienten mit akutem ischämischen Schlaganfall erlaubt. 


\subsection{Methodendiskussion}

\subsubsection{Studiendesign}

Der hauptsächliche Limitationsfaktor dieser Patientenstudie stellt der retrospektive Aufbau und das Fehlen einer Kontrollgruppe zum Vergleich der klinischneurologischen Verläufe dar. Die hier beschriebenen Ergebnisse können daher nur mit historischen Kontrollserien verglichen werden und es ist schwierig die klinischen Endergebnisse zu bewerten, da es keine vergleichbare Kontrollgruppe gibt (Schellinger und Hacke 2010). Gleichwohl ist die Bestätigung von Ergebnissen aus klinischen Trials in einem klinischen Setting und dazu in einer relativ großen Fallzahl essentiell für die Einführung eines neuen Instrumentes für die interventionelle Therapie des akuten Schlaganfalls (Leker und Liebeskind 2009). Zum Zeitpunkt der Publikation stellt das beschriebene Göttinger Patientenkollektiv die weltweit größte publizierte Single-Center-Untersuchung zur mechanischen Rekanalisation mit dem Penumbra System dar (Psychogios et al. 2012).

Abschließend sind diese Beobachtungen nicht unbedingt auf andere Arbeitsumgebungen übertragbar, da alle Interventionen von erfahrenen interventionellen Neuroradiologen durchgeführt wurden und die weitere Patientenversorgung auf einer neurologischen Intensivstation mit Schlaganfall-Erfahrung stattfand. Alle Interventionen wurden durch den Direktor oder die Oberärzte der neuroradiologischen Abteilung durchgeführt.

\subsubsection{Patientenauswahl}

Diese Arbeit umfasst eine Fallzahl 91 Patienten, welche aufgrund eines akuten ischämischen Schlaganfalls in der Abteilung für Neuroradiologie der Universitätsmedizin Göttingen durch Aspirationsthrombektomie mit Hilfe des Penumbra Systems therapiert wurden. Eine Limitation ist die Heterogenität der Verschlusslokalisationen und -längen in diesem Patientenkollektiv. Da für den Einsatz dieses neuartigen Verfahrens bisher keine offiziellen Leitlinien existieren, kann dies einen Einfluss auf die Auswahl des Patientenkollektivs haben.

Im Rahmen der Verlaufskontrolle konnten Daten von 80 Patienten eingeschlossen werden, dies hängt mit der Verlegung der Patienten zur Rehabilitation in periphere Kliniken zusammen. Die klinisch-neurologische Beurteilung erfolgte dort nicht immer standardisiert und der Einschluss der Verlaufsparameter für diese Patienten war somit nicht immer möglich. 


\subsubsection{Parameterauswahl}

Die Auswahl der erfassten Parameter zur vaskulären und klinischen Beurteilung orientiert sich an den von Higashida et al. vorgeschlagenen Standards zur Durchführung von Schlaganfallstudien mit intraarterieller Thrombolyse (Higashida et al. 2003). Die Festlegung des Follow-up-Zeitraumes von 90 Tagen folgt den bisher veröffentlichten Studien, um eine Vergleichbarkeit der Ergebnisse zu ermöglichen.

Während der NIHSS und der mRS sich bereits in sehr vielen Schlaganfall-Studien bewährt haben, steht der zur angiographischen Bewertung vielfach angewendete TIMI Score in der Kritik. Diese aus der kardialen Bildgebung stammende Skala erfasst die Rekanalisation des primären Gefäßverschlusses, was jedoch nicht gleichzusetzen ist mit der Reperfusion des distal gelegenen Gefäßbettes (Soares et al. 2009). Die Anwendung einer nicht exakt definierten TIMI-Skala im prospektiven, multizentrischen Penumbra Pivotal Stroke Trial wurde beanstandet und erlaubt keine exakte Vergleichbarkeit der Rekanalisationsergebnisse mit denen anderer Rekanalisationsinstrumente (Saver et al. 2010).

Vielfach wird die inkonsistente Verwendung der Begriffe „Revaskularisation“ und „Reperfusion“, als Endpunkte der Gefäßtherapie, kritisiert, was zu einem inkorrekten Vergleich von Rekanalisationsergebnissen verschiedener Studien führt (Alexandrov 2010). Rekanalisation bezieht sich auf die Wiederherstellung des Durchflusses am Ort der Okklusion, während die Reperfusion die distale Flusswiederherstellung zu den vom verschlossenen Gefäß versorgten Geweben definiert.

Den Empfehlungen des Stroke Therapy Academic Industry Roundtable (STAIR) folgend, wurden für diese Arbeit der $\mathrm{TICl}$ Score und der modified TIMI Score verwendet, beide sind an das komplexe zerebrale Gefäßsystem angepasst und finden zunehmend Verwendung in Arbeiten zur mechanischen Rekanalisation (Saver et al. 2009).

\subsection{Schlussfolgerung und Ausblick}

Das Hauptziel der akuten Schlaganfalltherapie ist die schnelle Wiederherstellung des zerebralen Blutflusses. Dies ist mit einem verbesserten klinischen Behandlungsergebnis und einer reduzierten Mortalitätsrate assoziiert (Rha und Saver 2007). 
Heutzutage ist die Schlaganfallbehandlung eine multimodale Therapieform, welche die Vorteile der sich schnell entwickelnden mechanischen Thrombektomie und der medikamentösen Thrombolyse miteinander kombiniert.

Die Verfügbarkeit neuartiger, effektiver Rekanalisationsinstrumente hat in den letzten Jahren das zur Verfügung stehende Therapiezeitfenster für Patienten bedeutend erweitert, bei denen die medikamentöse Lyse fehlgeschlagen oder kontraindiziert ist. Die bisher verfügbaren Studien und Fallsammlungen zeigen vielversprechende Resultate (siehe Tabelle 4, Seite 36/37).

\section{Gute Ergebnisse durch das Penumbra System}

Der Fokus auf mechanische Verfahren zur Gefäßrekanalisation, mit einer Abwendung von pharmakologischen Ansätzen, wurde maßgeblich durch die Veröffentlichung der ersten Studienergebnisse zum Penumbra System gelenkt. In der initialen einarmigen, prospektiven, Phase-1-Studie konnte erstmals eine Rekanalisationsrate von $100 \%$ bei 20 Patienten berichtet werden (Bose et al. 2008). Es konnte gezeigt werden, dass die Aspirationsthrombektomie mit dem Penumbra System als ein technisch sicheres und effektives Verfahren bei Schlaganfallpatienten innerhalb eines 8-Stunden-Zeitfensters ist. Die in dieser Arbeit beschriebenen Ergebnisse bestätigen die hohen Rekanalisationsraten im Vergleich zu früheren Verfahren und können zudem ein signifikant verbessertes Behandlungsergebnis bei rekanalisierten Patienten aufzeigen.

\section{Stentriever}

Seit 2010 sind sogenannte Stentriever, eine neue Generation von wieder entfernbaren intrakraniellen Stents, in der Erprobung und die Ergebnisse der ersten Pilotstudien deuten auf sehr hohe und schnell zu erreichende Rekanalisationsraten hin (Castano et al. 2010; Rohde et al. 2011). Das Zurückziehen eines entfalteten Stentrievers zur Durchführung einer Thrombektomie erhöht das Risiko von IntimaVerletzungen der Gefäßwand. Eine histopathologische Analyse von Gefäßpräparaten vom Schwein nach Trombektomie mit dem Trevo-Stentriever zeigte schwere Intimagefäßschäden; eine Blutung oder transmurale Dissektion wurde jedoch nicht nachgewiesen (Nogueira et al. 2011). Noch sind zu wenige Daten verfügbar, um eine verlässliche Aussage über die Zukunftsfähigkeit dieses neuen Konzeptes zu treffen. 


\section{Thrombushistologie}

Für die weitere Entwicklung von Thrombektomie-Instrumenten können auch die Thrombus-Eigenschaften von Bedeutung sein, da die histologischen Eigenschaften und die Pathophysiologie für die weitere Therapieentscheidung und Instrumentenwahl ausschlaggebend sein können. In einer kürzlich erschienenen Publikation wurde der Einfluss der Thrombus-Histologie auf das mechanische Rekanalisationsergebnis durch den MERCI Retriever untersucht. Dabei zeigte sich eine wesentlich langsamere und weniger erfolgreiche Thrombektomie bei fibrinreichen Thromben, wie sie bei thrombembolisch verursachten Schlaganfällen typisch sind, im Vergleich zu Erythrozyten-reichen Thromben (Yuki et al. 2011).

\section{Schlechtes Outcome, trotz guter Rekanalisationsrate}

In den Pilotstudien neuer Instrumente wird oft die hohe Rekanalisationsrate hervorgehoben, obwohl der wesentliche Endpunkt immer der Nutzen für den Patienten ist. Bei Analyse der verfügbaren Studien (siehe Tabelle 4, Seite 36/37) zur mechanischen Rekanalisation fällt auf, dass höhere Rekanalisationsraten im Zeitfenster von 3 - 8 Stunden nach Symptombeginn nicht zwingend zu einem besseren klinischen Endergebnis führen müssen (Coutts und Goyal 2009). Gründe hierfür können bei proximaler Rekanalisation eine persistierende periphere Okklusion sein (Rekanalisation ohne Perfusion) oder die Ausbildung eines ausgedehnten Infarkts bereits vor Therapiebeginn bei schlechter primärer Kollateralisierung.

Wird beispielsweise eine Rekanalisation, im Vergleich zu einer frühzeitig rekanalisierten identischen Verschlusssituation, mehrere Stunden später erzielt, kann man entweder von einer reduzierten Genesung ausgehen oder die Abhängigkeit von Rekanalisation und Therapieergebnis anzweifeln. Alle aktuellen Studien belegen, dass eine erfolgreiche Rekanalisation unerlässlich für den klinischen Behandlungserfolg ist (Rha und Saver 2007). Andererseits darf argumentiert werden, dass das funktionelle Ergebnis eines Patienten mit proximalem Gefäßverschluss und großem, mehrstündig bestehendem Penumbragewebe weniger von der Rekanalisation abhängt, als vielmehr von der Aufrechterhaltung einer ausreichenden Perfusion (Alexandrov 2010). 
$100 \%$ Rekanalisationsrate TIMI 2-3 $\square$ mRS nach 90 Tagen $\leq 2$

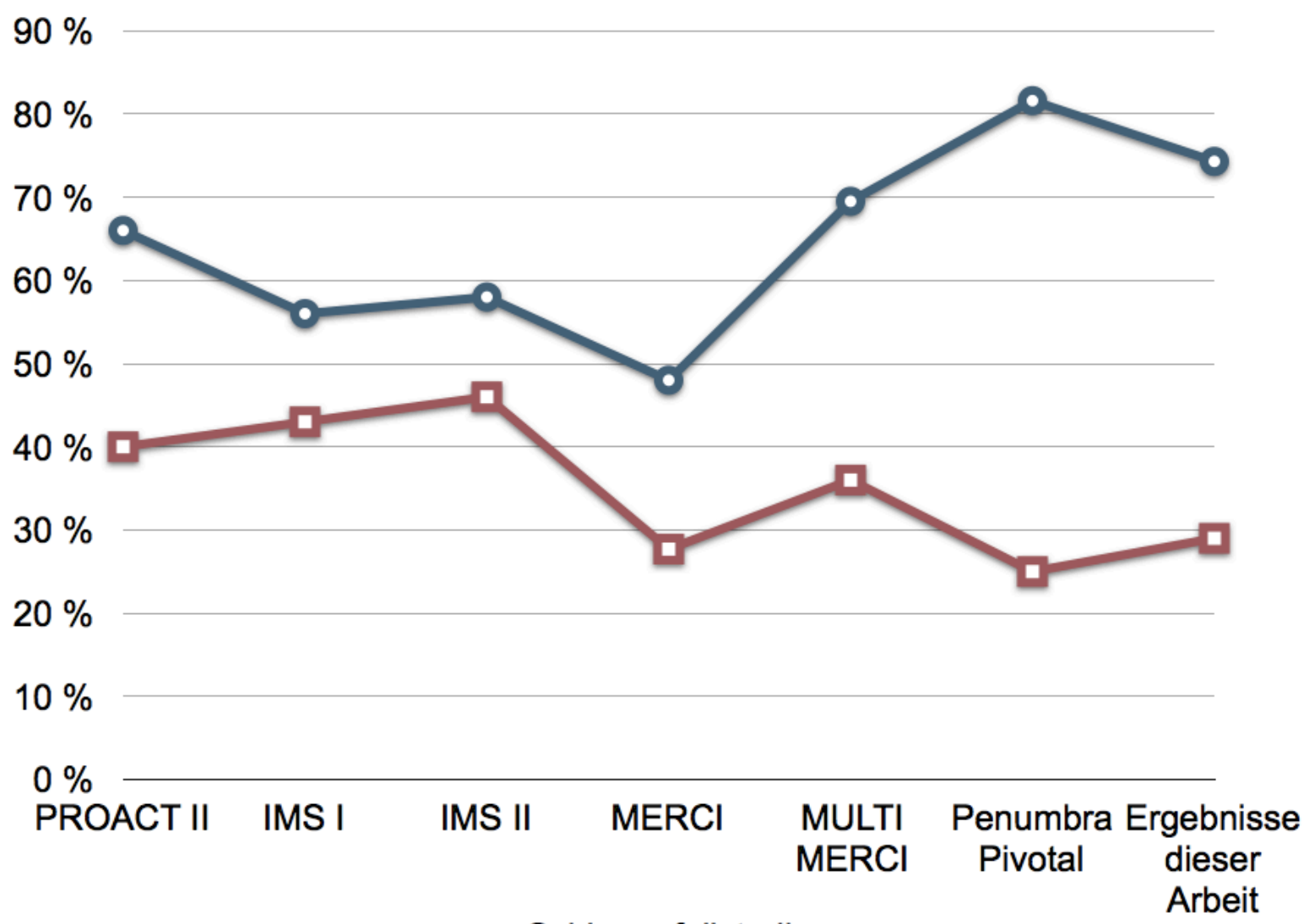

Schlaganfallstudien

Abbildung 34 - Schlaganfall-Therapieergebnis nach Rekanalisationsergebnis und mRS bisheriger Schlaganfallstudien. Obwohl durch neue Instrumente in den Studien zur intraarteriellen Thrombolyse und Thrombektomie höhere Rekanalisationsraten erzielt werden, konnte das gute neurologische Behandlungsergebnis (mRS $\leq 2)$ nach 90 Tagen bisher nicht in gleichem Maße gesteigert werden. Modifiziert nach Meyers et al. 2011, Seite 2595.

NIHSS: National Institutes of Health Scale; mRS: modified Rankin Scale; TIMI: Thrombolysis in Myocardial Infarction; PROACT: Prolyse in Acute Cerebral Thromboembolism Trial; IMS: Interventional Management of Stroke; MERCI: Mechanical Embolus Removal in Cerebral Ischemia.

Eine weitere Erklärung für die scheinbare Widersprüchlichkeit von hoher Rekanalisationsrate und gleichzeitig schlechtem Outcome findet sich in der inadäquaten Parameterauswahl vieler Schlaganfallstudien: Oftmals wurde lediglich die lokale Gefäßwiedereröffnung mittels TIMI Score bewertet und Fragmentationen, distale Embolisationen oder eine Verlagerung des Thrombus nicht in die Ergebnismessung mit einbezogen (Meyers et al. 2011). Aus diesem Grunde wurde für diese Arbeit maßgeblich der TICl-Score verwendet. 
Es wird sehr deutlich, wie wichtig die adäquate Patientenauswahl, besonders im Zeitfenster nach 3 bzw. 4,5 Stunden, für die Vertretbarkeit riskanter und kostenintensiver intraarterieller Eingriffe ist.

\section{Patientenauswahl}

Daher liegt zunehmend ein Forschungsschwerpunkt auf der optimalen Patientenauswahl für solche endovaskulären Therapien. Bisher existieren für die mechanische Thrombektomie keine Leitlinien und die Patientenauswahl erfolgt nach klinischen und bildgebenden Daten auf der Basis von Erfahrungswerten.

Hinsichtlich der Prognoseabschätzung bei systemischer Fibrinolyse findet verstärkt der ASPECT Score (Alberta Stroke Programme Early CT Scale) Anwendung (Pexman et al. 2001). Durch dieses Beurteilungssystem werden anhand von axialen CT-Schichtaufnahmen 10 anatomische Regionen auf Infarktfrühzeichen hin untersucht und erlauben eine weniger beobachterabhängige Evaluation als die Drittelregel bei Media-Infarzierungen. Patienten mit einem ASPECT Score von $<7$ haben ein erheblich schlechteres Rehabilitationsergebnis nach 3 Monaten und ein erhöhtes Blutungsrisiko (Demchuk et al. 2005). Goyal et al. führten retrospektiv eine Evaluation des ASPECT Scores mit den Daten des Penumbra Pivotal Stroke Trial durch, in welchem trotz einer Rekanalisationsrate von $81 \%$ nur bei $25 \%$ der Patienten ein gutes klinisches Behandlungsergebnis erzielt wurde. Es konnte gezeigt werden, dass Patienten mit einem ASPECT Score im initialen CT von $</=4$ nicht von einer Rekanalisationstherapie profitieren (Goyal et al. 2011).

Bezüglich endovaskulärer Therapien soll der THRIVE Score (Totaled Health Risks in Vascular Events Score), ein 10-stufiger prädiktiver Punktwert, welcher sich aus Patientenalter, Schweregrad des Schlaganfalls und chronischen Vorerkrankungen zusammensetzt, das zu erwartende klinische Behandlungsergebnis nach 3 Monaten zuverlässig prognostizieren (Flint et al. 2010). Auch der HIAT Score (Houston IAT Score) soll eine solche Entscheidungshilfe für ein interventionelles Vorgehen sein, jedoch benötigen beide Vorhersagewerte eine weitergehende Validierung (Ishkanian et al. 2011).

Ein weiterer vielversprechender Bildgebungs-basierter Ansatz zur Therapieauswahl von Patienten mit unbekanntem Symptombeginn (wake-up stroke) ist die Verwendung des DWI-FLAIR-Mismatch-Konzeptes. Schlaganfall-Patienten mit einer akuten ischämischen Läsion in der DWI-Sequenz, welche (noch) nicht in der FLAIR- 
Bildgebung detektierbar ist, sind wahrscheinlich innerhalb des Zeitfensters, in dem die Thrombolyse sicher und wirksam ist (Thomalla et al. 2011).

In der Zukunft werden voraussichtlich angepasste, patientenspezifische Kombinationen von Therapien zum Einsatz kommen, um das bestmögliche Ergebnis für den einzelnen Patienten zu erzielen (Saver 2011). Wie bereits erwähnt, scheint besonders im Zeitfenster jenseits von 3 Stunden eine präzise Patientenauswahl entscheidend, um Therapien individuell nach Nutzen und Risiken abwägen zu können (Alexandrov 2010).

Die ideale umfassende Behandlung des akuten ischämischen Schlaganfalls wird zukünftig somit nicht mehr nach einem reinen Zeitfenster-basierten Ansatz erfolgen. Vielmehr wird die Schlaganfallbehandlung sich in Zukunft pathophysiologisch an der Reperfusion rettenswerten Hirngewebes orientieren, sodass für unterschiedliche Patienten auch verschiedene therapeutische Zeitfenster gelten können (SernaCandel et al. 2010).

\section{Schlaganfall-Versorgungsstrukturen}

Neben einer zuverlässigen Patientenauswahl ist für den Einsatz neuartiger Thrombektomie-Instrumente, wie dem Penumbra System, ein erfahrenes und trainiertes Team von interventionellen Neuroradiologen erforderlich, das zusammen mit einer neurologischen Stroke-Unit optimal Schlaganfallpatienten therapieren kann. Bisher werden solche Verfahren nur an wenigen deutschen Kliniken (der Maximalversorgung) durchgeführt und es fehlt eine flächendeckende, koordinierte Versorgungsstruktur, wie sie beispielsweise für den Myokardinfarkt bereits erfolgreich etabliert wurde. Durch die begrenzte Anzahl an spezialisierten interventionell-neuroradiologischen Zentren mit 24h-Bereitschaft ist der strukturierte, schnelle Transport von Patienten mit der Verdachtsdiagnose Schlaganfall essentiell.

Ein weiterer wichtiger Punkt ist das Training der interventionell tätigen Neuroradiologen, sodass diese Routine im Einsatz eines Rekanalisationsinstrumentes, sowohl mit der technischen Handhabung als auch der möglichen Komplikationen, besitzen. Eine Evaluation der Daten dieser Arbeit bezüglich einer Lernkurve einzelner Interventionalisten wäre sicherlich sehr informativ und nützlich für die Implementierung zukünftiger neuer Instrumente und könnte wichtige Erkenntnisse zu erforderlichen Trainingsmaßnahmen liefern. 
Die Behandlungsabläufe können weiter optimiert werden, wobei dies bereits präklinisch durch eine breite Aufklärung der Bevölkerung zum Krankheitsbild des Schlaganfalls möglich ist. Neben schnelleren Rekanalisationsverfahren spielt besonders die Prozessoptimierung des klinischen Schlaganfallsmanagements eine wichtige Rolle. Verbesserte Abläufe in der Bildgebung, Entscheidungsfindung und Anästhesie können die Zeitverzögerung bis zum Interventionsbeginn deutlich reduzieren (Kreusch et al. 2012).

\section{Therapiekosten}

Bisher fehlt die Evidenz zur Beantwortung der Frage, ob die mechanische Thrombektomie der intraarteriellen und der intravenösen Fibrinolyse überlegen ist, besonders im Zeitfenster nach 3 bzw. 4,5 Stunden. Die Vertretbarkeit interventioneller, kostenintensiver Eingriffe stützt sich dabei auf den individuellen Heilversuch. In der aktuellen Diskussion zur mechanischen Rekanalisation von Schlaganfallpatienten findet zunehmend die Kosteneffektivität Erwähnung und ist aufgrund der hohen gesundheitsökonomischen Bedeutung des Schlaganfalls von Interesse. Eine amerikanische Studie untersuchte anhand eines theoretischen Modells die reine Kosteneffektivität der mechanischen Therapie im Vergleich zur intravenösen Lyse jenseits des 3-Stunden-Zeitfensters (Nguyen-Huynh und Johnston 2011). Basierend auf dem amerikanischen Gesundheitssystem ergibt sich eine Kosteneffektivität ab einer Rekanalisationsrate von $\geq 67 \%$. Weitere Modellberechnungen kamen zu ähnlichen Ergebnissen (Kim AS et al. 2011; Patil et al. 2009). Diese Ergebnisse können nicht ohne Weiteres auf das deutsche Gesundheitssystem übertragen werden und sie dürfen keinesfalls als zusätzliche Evidenz der Wirksamkeit der Thrombektomie-Behandlung interpretiert werden. Dies kann nur durch eine randomisierte Studie erbracht werden.

\section{Zulassungskriterien und der Mangel an randomisiert-kontrollierten Studien}

Die mechanischen Rekanalisationsverfahren befinden sich in einer rasanten Entwicklung und vielversprechende Ansätze lassen die Implementierung in die standardisierte Schlaganfallbehandlung als Ergänzung zur medikamentösen Therapie erhoffen. 
Vielfache Kritik wird an der Zulassungspraxis für neue Thrombektomieinstrumente geübt (Schellinger und Hacke 2010): Der MERCI Retriever und das Penumbra System wurden von der amerikanischen Food and Drug Association (FDA) für die Thrombusentfernung in Hirnarterien bei akutem ischämischen Schlaganfall zugelassen. Für die FDA-Zulassung ist lediglich die technische Machbarkeit und Sicherheit vorzuweisen. Vorhandene Patientendaten stammen größtenteils aus retrospektiven, nicht-kontrollierten Studien mit kleiner Fallzahl. Dennoch kommen die neuen Instrumente in interventionell-neuroradiologischen Zentren vielfach zum Einsatz für Patienten, die von der rt-PA-Gabe ausgeschlossen sind oder wo diese keinen Erfolg brachte. Basierend auf heutigen Erkenntnissen ist ein proaktives Therapieregime auch gerechtfertigt, da ein langstreckiger Hirngefäßverschluss im natürlichen Verlauf eine verheerende Prognose hat. Eine Etablierung der mechanischen Thrombektomie als Therapie der Wahl scheint für proximale und langstreckige Gefäßverschlüsse, welche ansonsten mit einer fatalen Prognose einhergehen, wahrscheinlich. Für kurzstreckige und sehr distale Verschlüsse erscheint dies weniger wahrscheinlich, da hier die intravenöse Fibrinolyse gute Therapieergebnisse ermöglicht und ein intraarterielles Vorgehen hier aufgrund der empfindlichen Gefäßanatomie größere Risiken birgt.

Dringend werden randomisierte, kontrollierte Studien mit klinischen Ergebnisparametern als primäre Endpunkte gefordert, um den eindeutigen Nachweis eines erweiterten therapeutischen Nutzens für die Patienten durch die mechanische Thrombektomie, im Vergleich zur medikamentösen Thrombolyse, erbringen zu können (Coutts und Goyal 2009; Saver 2011; Schellinger und Hacke 2010).

Die Schwierigkeit liegt im Fehlen eines allgemein anerkannten peri-interventionellen Protokolls (Gralla et al. 2012): Die Anzahl der zur Verfügung stehenden Instrumente, sich daraus ergebende Kombinationsmöglichkeiten, zusätzliche Variationen mit intravenösen und/oder intraarteriellen Thrombolytika, verschiedene Sedationsformen (Lokalanästhesie oder Vollnarkose) sowie weitere adjuvante Therapien (Thrombozytenaggregationshemmer, Heparin, Glykoprotein Ilb/Illa-Antagonisten) sind nahezu unüberschaubar. Das Stroke Therapy Academic Industry Roundtable (STAIR) Committee hat deshalb Empfehlungen für zukünftige Studiendesigns veröffentlicht, um die Entwicklung und standartisierte Evaluation neuer Schlaganfalltherapien zu unterstützen (Saver et al. 2009). 
Neben einer sehr großen Zahl an klinischen Studien zu einzelnen neuen Rekanalisationsinstrumenten, wurden auch Studien zu Kombinationstherapien bei akutem Schlaganfall initiiert (siehe Tabelle 8, Seite 98). Besonders hohe Erwartungen betreffen die randomisiert, kontrollierte Interventional Management of Stroke III Study (IMS III), welche das klinische Behandlungsergebnis bei 900 Patienten innerhalb des 3-Stunden-Zeitfensters von intravenöser Lyse oder der kombinierten intravenösen Lyse und intraarteriellen Thrombolyse/Thrombektomie vergleichen will (Khatri et al. 2008). Die Magnetic Resonance and Recanalization of Stroke Clots Using Embolectomy Study (MR RESCUE) will die systemische Lyse mit der reinen mechanischen Thrombektomie mittels Penumbra System oder MERCI Retriever bei Patienten im 8-Stunden-Zeitfenster vergleichen und zielt auf die Patientenauswahl durch MRT-Perfusionsbildgebung.

Speziell für das Penumbra System soll bis Anfang 2015 in der THERAPY-Studie die Sicherheit und Effektivität des Penumbra Systems als Begleitherapie zur intravenösen rt-PA-Applikation bei Patienten mit einem akuten, langstreckigen (> 8mm) Hirngefäßverschluss getestet werden und eine Überlegenheit gegenüber alleiniger intravenöser Fibrinolyse hinsichtlich des klinischen Behandlungsergebnisses nach 90 Tagen bewiesen werden.

Von Interventionalisten werden ethische Bedenken bezüglich einer StudienRandomisierung von (jungen) Schlaganfallpatienten geäußert, wenn bei Patienten eine intravenöse Thrombolyse nicht erfolgsversprechend ist und sie gleichzeitig dramatische Verbesserung bei Patienten nach mechanischer Thrombektomie mit eigenen Augen gesehen haben. Nichtsdestotrotz, die Randomisierung von Patienten in klinischen Studien ist eine vernünftige und angemessene Vorangehensweise und es gibt keinen anderen Weg, um dieses neue, vielversprechende Therapiekonzept in der Schlaganfallbehandlung mit weit verbreiteter Akzeptanz zu etablieren (Kreusch et al. 2012; Nguyen et al. 2011). 


\begin{tabular}{|c|c|c|c|c|c|}
\hline \multicolumn{6}{|c|}{ Laufende Studien zur Therapie des akuten ischämischen Schlaganfalls } \\
\hline Studie & Zeitraum & $\begin{array}{c}\text { Geplante } \\
\text { Patienten- } \\
\text { zahl }\end{array}$ & Zeitfenster & $\begin{array}{l}\text { Studien- } \\
\text { design }\end{array}$ & $\begin{array}{l}\text { Primärer } \\
\text { Endpunkt }\end{array}$ \\
\hline IMS III & $2006-2014$ & 900 & $0-3 \mathrm{~h}$ & $\begin{array}{c}\text { IV rt-PA vs. } \\
0.6 \mathrm{mg} / \mathrm{kg} \text { IV rt-PA } \\
\text { und IA (MERCl, } \\
\text { Penumbra System, } \\
\text { EKOS) }\end{array}$ & $\begin{array}{c}\text { mRS nach } 90 \\
\text { Tagen }\end{array}$ \\
\hline THRACE & $2010-2012$ & 480 & $0-3 \mathrm{~h}$ & $\begin{array}{l}\text { IV rtt-PA vs. } \\
0.9 \mathrm{mg} / \mathrm{kg} \text { IV rt-PA + } \\
\text { mechanische } \\
\text { Thrombektomie }\end{array}$ & $\begin{array}{c}\text { mRS nach } 90 \\
\text { Tagen }\end{array}$ \\
\hline $\begin{array}{c}\text { SYNTHESIS } \\
\text { EXP }\end{array}$ & $2008-2012$ & 350 & $\begin{array}{l}\text { IVT: } 0-3 \mathrm{~h} \\
\text { IAT: } 0-6 \mathrm{~h}\end{array}$ & $\begin{array}{l}\text { IV rt-PA vs. IA rt-PA } \\
\text { + mechanische } \\
\text { Thrombektomie }\end{array}$ & $\begin{array}{c}\text { mRS nach } 90 \\
\text { Tagen }\end{array}$ \\
\hline MR CLEAN & $2010-2015$ & 500 & $0-6 \mathrm{~h}$ & $\begin{array}{l}\text { IA rt-PA+/oder } \\
\text { mechanisch vs. kein } \\
\text { IA }\end{array}$ & $\begin{array}{c}\text { mRS nach } 90 \\
\text { Tagen }\end{array}$ \\
\hline $\begin{array}{c}\text { MR } \\
\text { RESCUE }\end{array}$ & $2004-2012$ & 120 & $0-8 \mathrm{~h}$ & $\begin{array}{l}\text { Standard vs. IA } \\
\text { (MERCI, Penumbra } \\
\text { System) mit MR- } \\
\text { Perfusion }\end{array}$ & $\begin{array}{c}\text { mRS nach } 90 \\
\text { Tagen }\end{array}$ \\
\hline DAWN & 2009 - k.A. & k.A. & $>8 \mathrm{~h}$ & $\begin{array}{l}\text { Standard vs. IA } \\
\text { (MERCI, Penumbra } \\
\text { System) mit CT- } \\
\text { Perfusion o. MRT- } \\
\text { Perfusion }\end{array}$ & $\begin{array}{c}\text { mRS nach } 90 \\
\text { Tagen }\end{array}$ \\
\hline SWIFT & $2010-2011$ & 200 & $0-8 \mathrm{~h}$ & MERCI vs. Solitaire & $\begin{array}{l}\text { Rekanalisation } \\
\text { TIMI } 2 \text { oder } 3\end{array}$ \\
\hline TREVO & $2010-2011$ & 60 & k.A. & Trevo & $\begin{array}{l}\text { Rekanalisation } \\
\text { TICI } 2 a\end{array}$ \\
\hline TREVO 2 & $2011-2011$ & 178 & k.A. & MERCI vs. Trevo & Rekanalisation \\
\hline PICS & $2009-2014$ & 2000 & k.A. & $\begin{array}{c}\text { IV rt-PA + Penumbra } \\
\text { System }\end{array}$ & $\begin{array}{c}\text { mRS nach } 90 \\
\text { Tagen }\end{array}$ \\
\hline THERAPY & $2012-2015$ & 692 & k.A. & $\begin{array}{l}\text { IV rt-PA vs. IV rt-PA } \\
+ \text { + Penumbra System }\end{array}$ & $\begin{array}{c}\text { mRS nach } 90 \\
\text { Tagen }\end{array}$ \\
\hline START & $2009-2012$ & 200 & $0-8 \mathrm{~h}$ & $\begin{array}{l}\text { IV rt-PA + Penumbra } \\
\text { System }\end{array}$ & $\begin{array}{c}\text { mRS nach } 90 \\
\text { Tagen }\end{array}$ \\
\hline $\begin{array}{l}\text { WAKE UP } \\
\text { STROKE }\end{array}$ & $2010-2012$ & 40 & $\begin{array}{l}<24 \mathrm{~h} \text { nach } \\
\text { Symptombeginn }\end{array}$ & $\begin{array}{l}\text { IV rt-PA bis } 0.9 \\
\mathrm{mg} / \mathrm{kg}\end{array}$ & $\mathrm{SICH}$ in $24 \mathrm{~h}$ \\
\hline $\begin{array}{c}\text { MR } \\
\text { WITNESS }\end{array}$ & $2010-2012$ & 80 & $0-4,5 \mathrm{~h}$ & $\begin{array}{l}\text { IV rt-PA bis } 0.9 \\
\text { mg/kg nach MRT- } \\
\text { Kriterien }\end{array}$ & $\begin{array}{c}\text { rt-PA- } \\
\text { Anwendungs- } \\
\text { sicherheit }\end{array}$ \\
\hline
\end{tabular}

Tabelle 8 - Laufende Studien zur Therapie des akuten ischämischen Schlaganfalls.

Nach Baker et al. (2011) und Nguyen et al. (2011). 


\section{Zusammenfassung}

\section{Hintergrund und Zweck}

Das Penumbra System ist ein innovatives, neuartiges mechanisches Instrument für die Rekanalisierung von langstreckigen Gefäßverschlüssen der Hirnarterien durch Thrombus-Aspiration. Zweck dieser Studie ist die Beurteilung der Effektivität der Gefäßrekanalisation und des neurologischen Endergebnisses von Patienten, die infolge eines akuten ischämischen Schlaganfalls mit dem Penumbra System therapiert wurden.

\section{Methoden}

Insgesamt 91 Patienten mit akutem ischämischen Schlaganfall aufgrund eines Verschlusses hirnversorgender Arterien wurden mit dem Penumbra System behandelt und in diese retrospektive Studie aufgenommen. In 14 Fällen kam dabei allein das Penumbra System zum Einsatz, bei weiteren 77 Patienten wurde die mechanische Rekanalisation mit intraarterieller und/oder intravenöser Thrombolyse kombiniert. Das klinische Endergebnis wurde anhand des modified Rankin Scale (mRS) bewertet; die Rekanalisation wurde mit dem Thrombolysis in Cerebral Infarction Score (TICI Score) beurteilt.

\section{Ergebnisse}

Das durchschnittliche Patientenalter betrug $62 \pm 19,4$ Jahre; der mittlere NIH Stroke Scale (NIHSS) bei Krankenhausaufnahme war 17. Eine erfolgreiche Rekanalisation konnte bei $77 \%$ der Patienten erreicht werden. Durchschnittlich vergingen 49 Minuten von arterieller Punktion bis zur Gefäßrekanalisation (Quartillen 31 - 86). In der Verlaufskontrolle zeigten $36 \%$ der Patienten eine NIHSS-Verbesserung von $\geq 10$ Punkten und $34 \%$ der Patienten mit einem Verschluss in der vorderen Zirkulation hatten einen $m R S \geq 2$, während nur $7 \%$ der Patienten mit einem Gefäßverschluss in der hinteren Zirkulation ein gutes Endergebnis im Nachbeobachtungszeitraum vorwiesen. Insgesamt 20 Patienten verstarben während des Krankenhausaufenthaltes; kein Todesfall war auf den Einsatz des Penumbra Systems zurückzuführen. 


\section{Fazit}

Zusammenfassend bestätigen die in dieser Arbeit dargestellten Ergebnisse einer retrospektiven Single-Center-Studie die Effektivität des Penumbra Systems für die mechanische Rekanalisation von ischämischen Schlaganfallpatienten.

Eine erfolgreiche und schnelle Rekanalisation mithilfe des Penumbra Systems ist dabei assoziiert mit einer signifikanten Verbesserung des funktionellen-klinischen Endergebnisses bei Patienten mit akutem ischämischen Schlaganfall aufgrund eines Gefäßverschlusses der hirnversorgenden Gefäße.

Der erweiterte klinische Nutzen der mechanischen Thrombektomie, im Vergleich zur medikamentösen Standardtherapie, muss in der Zukunft durch eine prospektive, randomisierte und kontrollierte Studie belegt werden. 


\section{Anhang}

\section{Anhang 1: National Institute of Health Stroke Scale (NIHSS)}

\begin{tabular}{|c|c|}
\hline \multicolumn{2}{|l|}{ NIHSS (0-42 Punkte) } \\
\hline $\begin{array}{l}\text { Neurologische } \\
\text { Parameter }\end{array}$ & NIHSS-Punktwert / Befund \\
\hline 1. Bewusstseinslage & $\begin{array}{l}0=\text { wach } \\
1=\text { somnolent }(\text { Reaktion auf geringe Stimuli) } \\
2=\text { stuporös (Reaktion auf wiederholte, starke Stimuli) } \\
3=\text { komatös }\end{array}$ \\
\hline $\begin{array}{l}\text { 2. Orientierung } \\
\text { Alter? Monat? }\end{array}$ & $\begin{array}{l}0=\text { beide Antworten richtig } \\
1=\text { eine Antwort richtig } \\
2=\text { keine Antwort richtig }\end{array}$ \\
\hline $\begin{array}{l}\text { 3. Aufforderungen } \\
\text { Augen öffnen, Augen } \\
\text { schließen }\end{array}$ & $\begin{array}{l}0=\text { beide Aufforderungen korrekt befolgt } \\
1=\text { eine Aufforderung korrekt befolgt } \\
2=\text { keine Aufforderung korrekt befolgt }\end{array}$ \\
\hline 4. Blickwendung & $\begin{array}{l}0=\text { normal } \\
1=\text { partielle Parese } \\
2=\text { forcierte Deviation }\end{array}$ \\
\hline $\begin{array}{l}\frac{\text { 5. Gesichtsfeld }}{\text { geprüft werden alle vier }} \\
\text { Quadranten }\end{array}$ & $\begin{array}{l}0=\text { normal } \\
1=\text { Quadrantenanopsie oder visueller Neglect } \\
2=\text { komplette Hemianopsie } \\
3=\text { bilaterale Hemianopsie oder Blindheit }\end{array}$ \\
\hline$\underline{\text { 6. Mimik }}$ & $\begin{array}{l}0=\text { normal } \\
1=\text { geringe Assymetrie (Mundast) } \\
2=\text { partielle faziale Parese (Mundast) } \\
3=\text { komplette faziale Parese }\end{array}$ \\
\hline $\begin{array}{l}\text { 7. Armmotorik } \\
\text { für beide Arme getrennt, } \\
\text { je } 10 \text { Sekunden }\end{array}$ & $\begin{array}{l}0=\text { kein Absinken (oder Amputation) } \\
1=\text { Absinken in } 10 \text { Sekunden } \\
2=\text { sinkt auf Unterlage, Anheben möglich } \\
3=\text { kein Anheben gegen Schwerkraft } \\
4=\text { Plegie }\end{array}$ \\
\hline $\begin{array}{l}\text { 8. Beinmotorik } \\
\text { für beide Beine getrennt, } \\
\text { je } 10 \text { Sekunden }\end{array}$ & $\begin{array}{l}0=\text { kein Absinken (o. Amputation) } \\
1=\text { Absinken in } 5 \text { Sekunden } \\
2=\text { sinkt auf Unterlage, Anheben möglich } \\
3=\text { kein Anheben gegen Schwerkraft } \\
4=\text { Plegie }\end{array}$ \\
\hline 9. Ataxie & $\begin{array}{l}0=\text { normal oder wegen Paresen nicht zu Beurteilen } \\
1=\text { in einer Extremität } \\
2=\text { in zwei oder mehr Extremitäten }\end{array}$ \\
\hline 10. Sensibilität & $\begin{array}{l}0=\text { normal } \\
1=\text { partieller Sensibilitätsverlust } \\
2=\text { schwerer bis vollständiger Verlust }\end{array}$ \\
\hline 11. Sprache & $\begin{array}{l}0=\text { keine Aphasie } \\
1=\text { Einschränkung von Wortflüssigkeit } / \text { Verständnis } \\
2=\text { schwere Aphasie, fragmentierter Ausdruck } \\
3=\text { globale Aphasie oder stumm }\end{array}$ \\
\hline 12. Sprechen & $\begin{array}{l}0=\text { normal (oder intubiert) } \\
1=\text { verwaschen, aber verständlich } \\
2=\text { unverständlich oder stumm }\end{array}$ \\
\hline 13. Neglect & $\begin{array}{l}0=\text { normal } \\
1=\text { partieller Neglect (unimodal) } \\
2=\text { kompletter Neglect (multimodal) }\end{array}$ \\
\hline
\end{tabular}


Anhang 2: Modified Rankin Scale (mRS)

\begin{tabular}{|cl|}
\hline mRS (0-6 Punkte) & \\
\hline mRS-Grad & mRS-Punktwert / Befund \\
\hline 0 & $\begin{array}{l}\text { Keine Symptome } \\
\text { Konnte alle gewohnten Aufgaben und Aktivitäten verrichten. } \\
\text { Keine signifikanten Einschränkungen. } \\
\text { Unfähig alle früheren Aktivitäten zu verrichten, erledigt die } \\
\text { eigenen Angelegenheiten ohne fremde Hilfe } \\
\text { Bedurfte einiger Unterstützung, war aber in der Lage, Lage } \\
\text { ohne Hilfe zu gehen fün die }\end{array}$ \\
2 & $\begin{array}{l}\text { Unfähig ohne Hilfe zu gehen, und unfähig ohne Hilfe für die } \\
\text { eigenen Bedürfnisse zu sorgen }\end{array}$ \\
\hline 3 & $\begin{array}{l}\text { Bettlägerig, inkontinent, bedurfte ständiger Pflege und } \\
\text { Aufmerksamkeit }\end{array}$ \\
\hline 6 & $\underline{\text { Tod }}$ \\
\hline
\end{tabular}

\section{Anhang 3: Modified Thrombolysis In Myocardial Infarction (mTIMI)} Bewertungsskala

\begin{tabular}{|cl|}
\hline mTIMI (0-4 Punkte) & \\
\hline mTIMI-Grad & Beschreibung \\
\hline 0 & Kein Fluss \\
1 & $\begin{array}{l}\text { Etwas Kontrastmittelpenetration hinter den Verschlussort, } \\
\text { aber kein Fluss hinter dem Gefäßverschluss }\end{array}$ \\
2 & $\begin{array}{l}\text { Distale Perfusion, aber verlangsamte Füllung in allen Gefäßen } \\
\text { Distale Perfusion mit adäquater Perfusion in weniger als der } \\
\text { Hälfte der distalen Gefäße } \\
\text { Distale Perfusion mit adäquater Perfusion in mehr als der } \\
\text { Hälfte der distalen Gefäße }\end{array}$ \\
\hline
\end{tabular}

\section{Anhang 4: Thrombolysis in Cerebral Infarction (TICl) Bewertungsskala}

\begin{tabular}{|c|c|}
\hline \multicolumn{2}{|c|}{ TICI (0-3 Punkte) } \\
\hline TICI-Grad & Beschreibung \\
\hline 0 & 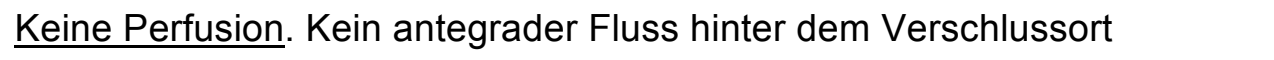 \\
\hline 1 & $\begin{array}{l}\text { Penetration mit minimaler Perfusion. Das Kontrastmittel passiert den } \\
\text { Verschlussort, füllt aber nicht das gesamte zerebrale Gefäßbett distal des } \\
\text { Verschlusses für die Dauer der angiografischen Serie aus }\end{array}$ \\
\hline 2 & $\begin{array}{l}\text { Partielle Perfusion. Das Kontrastmittel passiert den Verschlussort und füllt } \\
\text { das arterielle Gefäßbett distal des Verschlusses aus; jedoch ist der }\end{array}$ \\
\hline
\end{tabular}




\begin{tabular}{|cl}
\hline Kontrastmittelzufluss in das Gefäß distal des Verschlusses und / oder der \\
Kontrastmittelabfluss aus dem distalen Gefäß merklich langsamer als der \\
Kontrastmittelzu- oder -abfluss aus vergleichbaren Gebieten, die nicht \\
durch das verschlossene Gefäß perfundiert werden, z. B. die \\
kontralaterale Hirnarterie oder das Gefäßbett proximal des \\
Gefäßverschlusses
\end{tabular}

\section{Anhang 5: ECASS-Klassifikation der intrakraniellen Hämorrhagien}

\begin{tabular}{|ccl|}
\hline ICH-Klassifikation nach ECASS (European Cooperative Acute Stroke Study) \\
\hline \multicolumn{2}{|c|}{ Grad } & Beschreibung \\
\hline $\begin{array}{c}\text { Hämmorrhagische } \\
\text { Infarkte }(\mathrm{HI})\end{array}$ & $\mathrm{HI}-1$ & $\begin{array}{l}\text { Kleine Petechien entlang der Infarktgrenzen } \\
\text { mehr konfluierende Petechien (HI-2) innerhalb des } \\
\text { Infarktbereiches ohne Verdrängungseffekt }\end{array}$ \\
\hline $\begin{array}{c}\text { Parenchymale } \\
\text { Hämatome }(\mathrm{PH})\end{array}$ & $\mathrm{PH}-1$ & $\begin{array}{l}\text { Hämatom in } \leq 30 \% \text { des infarzierten Areals mit geringem } \\
\text { Verdrängungseffekt } \\
\text { Hämatom in > } 30 \% \text { des infarzierten Areals mit } \\
\text { signifikantem Verdrängungseffekt oder hämmorrhagische } \\
\text { Läsion außerhalb des infarzierten Areals }\end{array}$ \\
\hline
\end{tabular}




\section{Abbildungsverzeichnis}

Abbildung 1: $\quad$ Übersicht der supraaortalen Gefäße $\quad$ Seite 7

Abbildung 2: Übersicht der intrakraniellen vorderen Zirkulation in Seite 9 anteroposteriorer Sicht

Abbildung 3: Übersicht der intrakraniellen vorderen Zirkulation in lateraler $\quad$ Seite 9 Sicht

Abbildung 4: $\quad$ Übersicht der intrakraniellen hinteren Zirkulation in 10 anteroposteriorer Sicht

Abbildung 5: $\quad$ Übersicht der intrakraniellen hinteren Zirkulation in lateraler $\quad$ Seite 11 Sicht

Abbildung 6: Übersicht über den Circulus arteriosus Willisii $\quad$ Seite 11

Abbildung 7: Schematische Darstellung des Konzeptes der ischämischen Seite 17 Penumbra

Abbildung 8: Darstellung eines Schlaganfall-CT-Protokolls Seite 19

Abbildung 9: $\quad$ Flussdiagramm eines möglichen Ablaufes einer Seite 22 neuroradiologischen Schlaganfall-Akutdiagnostik mittels MRT

Abbildung 10: Übersicht über verschiedene Instrumente zur mechanischen Seite 32 Rekanalisation

Abbildung 11: Flussdiagramm einer möglichen Diagnostik und

Seite 35

Akutversorgung eines ischämischen Schlaganfalls im vorderen Gefäßkreislauf

Abbildung 12: Schematisches Diagramm des neurovaskulären Gefäßbettes

Seite 39 und der möglichen Reperfusionsstrategien

Abbildung 13: Zusammenhang zwischen Reperfusion, Gefäßkollateralisation, der Zeit und dem klinischen Endergebnis bei akutem ischämischen Schlaganfall

Abbildung 14: Prinzip der digitalen Subtraktionsangiographie

Abbildung 15: Angiographiesystem „AXIOM Artis dBa“

Abbildung 16: Verfügbare Größen des Penumbra Systems 
Systems

Abbildung 18: Ablauf der mechanischen Rekanalisation mit dem Penumbra Seite 55 System

Abbildung 19: Alters- und Geschlechtsverteilung des behandelten

Seite 62

Patientenkollektivs

Abbildung 20: Verteilung der präinterventionellen Graduierungspunkte des

Seite 63 NIHSS

Abbildung 21: Verteilung der präinterventionellen Graduierungspunkte des

Seite 63 mRS

Abbildung 22: Zeitverlauf von Symptombeginn bis Gefäßrekanalisation für die

Seite 64 vordere und hintere Zirkulation

Abbildung 23: Übersicht über die Lokalisationen der Gefäßverschlüsse

Seite 65

Abbildung 24: Übersicht über die Seitenverteilung der Gefäßverschlüsse auf

Seite 65 die Hemisphären

Abbildung 25: Häufigkeiten des Einsatzes von intravenöser und intraarterieller

Seite 67 Fibrinolyse sowie reiner mechanischer Thrombektomie

Abbildung 26: Prä- und postinterventioneller Gefäßstatus des

Seite 70 Patientenkollektivs

Abbildung 27: Postinterventionelle Rekanalisationsraten nach

Seite 71 Verschlusslokalisation

Abbildung 28: Darstellung des mittleren NIHSS-Verlaufes im

Seite 74 Beobachtungszeitraum

Abbildung 29: Darstellung der Prozentzahl an Patienten mit einem mRS $\leq 2$

Seite 74 während des Beobachtungszeitraumes

Abbildung 30: Darstellung des klinischen Behandlungsergebnisses im

Seite 74 Gesamtkollektiv und in den Subgruppen

Abbildung 31: Darstellung des klinischen Behandlungsergebnisses der rekanalisierten Patienten

Abbildung 32: Fallbeispiel 1 der Aspirationstherapie mittels Penumbra System

Seite 78

Abbildung 33: Fallbeispiel 2 der Aspirationstherapie mittels Penumbra System

Seite 80

Abbildung 34: Schlaganfall-Therapieergebnis nach Rekanalisationsergebnis

Seite 92 und $\mathrm{mRS}$ bisheriger Schlaganfallstudien 


\section{Tabellenverzeichnis}

Tabelle 1: Übersicht über das notfallmedizinische Management der

Seite 25

Vitalparameter beim ischämischen Schlaganfall

Tabelle 2: Wichtigste Ein- und Ausschlusskriterien der Zulassungsstudien für

Seite 27 die IVT

Tabelle 3: Übersicht über die möglichen Therapieformen in Kombination mit

Seite 35 dem Penumbra System

Tabelle 4: Studienergebnisse zur Therapie des akuten ischämischen

Seite 36

Schlaganfalls - Teil 1

Studienergebnisse zur Therapie des akuten ischämischen

Seite 37

Schlaganfalls - Teil 2

Tabelle 5: Technische Daten des Penumbra Systems

Seite 52

Tabelle 6: Präinterventioneller Gefäßstatus der Patienten entsprechend der

Seite 64 Graduierungssysteme TICI und mTIMI

Tabelle 7: Postinterventionelle intrakranielle Blutungen

Seite 69

Tabelle 8: Laufende Studien zur Therapie des akuten ischämischen

Seite 98 Schlaganfalls 


\section{Literaturverzeichnis}

Adams HP, Bendixen BH, Kappelle LJ, Biller J, Love BB, Gordon DL, Marsh EE (1993): Classification of subtype of acute ischemic stroke. Definitions for use in a multicenter clinical trial. TOAST. Trial of Org 10172 in Acute Stroke Treatment. Stroke 24(1): 3541

Adams HP, Davis PH, Leira EC, Chang KC, Bendixen BH, Clarke WR, Woolson RF, Hansen MD (1999): Baseline NIH Stroke Scale score strongly predicts outcome after stroke: A report of the Trial of Org 10172 in Acute Stroke Treatment (TOAST). Neurology 53(1): 126-131

Adams HP, del Zoppo G, Alberts MJ, Bhatt DL, Brass L, Furlan A, Grubb RL, Higashida RT, Jauch EC, Kidwell C, et al. (2007): Guidelines for the early management of adults with ischemic stroke: a guideline from the American Heart Association/American Stroke Association Stroke Council, Clinical Cardiology Council, Cardiovascular Radiology and Intervention Council, and the Atherosclerotic Peripheral Vascular Disease and Quality of Care Outcomes in Research Interdisciplinary Working Groups: The American Academy of Neurology affirms the value of this guideline as an educational tool for neurologists. Circulation 115(20): 478-534

Albers GW, Olivot JM (2007): Intravenous alteplase for ischaemic stroke. Lancet $\underline{369}(9558)$ : 249-250

Albers GW, Thijs VN, Wechsler L, Kemp S, Schlaug G, Skalabrin E, Bammer R, Kakuda W, Lansberg MG, Shuaib A, et al. (2006): Magnetic resonance imaging profiles predict clinical response to early reperfusion: the diffusion and perfusion imaging evaluation for understanding stroke evolution (DEFUSE) study. Ann Neurol 60(5): 508-517

Alexandrov AV (2010): Current and future recanalization strategies for acute ischemic stroke. J Intern Med 267(2): 209-219

Alexandrov AV, Demchuk AM, Felberg RA, Christou I, Barber PA, Burgin WS, Malkoff M, Wojner AW, Grotta JC (2000): High rate of complete recanalization and dramatic clinical recovery during tPA infusion when continuously monitored with 2-MHz transcranial doppler monitoring. Stroke $\underline{31}(3): 610-614$

Alexandrov AV, Molina CA, Grotta JC, Garami Z, Ford SR, Alvarez-Sabin J, Montaner J, Saqqur M, Demchuk AM, Moye LA, et al. (2004): Ultrasound-enhanced systemic thrombolysis for acute ischemic stroke. N Engl J Med 351(21): 2170-2178

Andre C (2002): The NIH Stroke Scale is unreliable in untrained hands. J Stroke Cerebrovasc Dis 11(1): 43-46

Anxionnat R, Bracard S, Macho J, Da Costa E, Vaillant R, Launay L, Trousset $\mathrm{Y}$, Romeas R, Picard L (1998): 3D angiography. Clinical interest. First applications in interventional neuroradiology. J Neuroradiol 25(4): 251-262

Arnold M, Nedeltchev K, Remonda L, Fischer U, Brekenfeld C, Keserue B, Schroth G, Mattle HP (2005): Recanalisation of middle cerebral artery occlusion after intra-arterial thrombolysis: different recanalisation grading systems and clinical functional outcome. J Neurol Neurosurg Psychiatry $\underline{76}(10):$ 1373-1376 
Astrup J, Siesjo BK, Symon L (1981): Thresholds in cerebral ischemia - the ischemic penumbra. Stroke 12(6): 723-725

AWMF-Leitlinien: Leitlinien für Diagnostik und Therapie in der Neurologie. Georg Thieme Verlag, Stuttgart 2008

Baker WL, Colby J A, Tongbram V, Talati R, Silverman IE, White CM, Kluger J, Coleman CI (2011): Neurothrombectomy devices for the treatment of acute ischemic stroke: state of the evidence. Ann Intern Med 154(4): 243-252

Bamford JM, Sandercock PA, Warlow CP, Slattery J (1989): Interobserver agreement for the assessment of handicap in stroke patients. Stroke 20(6): 828

Banks JL, Marotta CA (2007): Outcomes validity and reliability of the modified Rankin scale: implications for stroke clinical trials: a literature review and synthesis. Stroke $\underline{38}(3)$ : 1091-1096

Barber PA, Zhang J, Demchuk AM, Hill MD, Buchan AM (2001): Why are stroke patients excluded from TPA therapy? An analysis of patient eligibility. Neurology $\underline{56}(8)$ : 10151020

Baron JC (1999): Mapping the ischaemic penumbra with PET: implications for acute stroke treatment. Cerebrovasc Dis $\underline{9}(4): 193-201$

Bendszus M, Koltzenburg M, Burger R, Warmuth-Metz M, Hofmann E, Solymosi L (1999): Silent embolism in diagnostic cerebral angiography and neurointerventional procedures: a prospective study. Lancet 354(9190): 1594-1597

Blackham KA (2011): Extensive dural sinus thrombosis: successful recanalization with thrombolysis and a novel thrombectomy device. J Neurosurg 114(1): 133-135

Bose A, Henkes H, Alfke K, Reith W, Mayer TE, Berlis A, Branca V, Sit SP (2008): The Penumbra System: a mechanical device for the treatment of acute stroke due to thromboembolism. Am J Neuroradiol 29(7): 1409-1413

Brekenfeld C, Schroth G, Mattle HP, Do DD, Remonda L, Mordasini P, Arnold M, Nedeltchev K, Meier N, Gralla J (2009): Stent placement in acute cerebral artery occlusion: use of a self-expandable intracranial stent for acute stroke treatment. Stroke $\underline{40}(3)$ : 847-852

Brice JH, Griswell JK, Delbridge TR, Key CB (2002): Stroke: from recognition by the public to management by emergency medical services. Prehosp Emerg Care $\underline{6}(1): 99-106$

Brott T, Adams HP, Olinger CP, Marler JR, Barsan WG, Biller J, Spilker J, Holleran R, Eberle R, Hertzberg V, et al. (1989): Measurements of acute cerebral infarction: a clinical examination scale. Stroke 20(7): 864-870

Caplan LR: Caplan's Stroke: A Clinical Approach. 4th Edition. Saunders, Philadelphia 2009

Castano C, Dorado L, Guerrero C, Millan M, Gomis M, Perez de la Ossa N, Castellanos M, Garcia MR, Domenech S, Davalos A (2010): Mechanical thrombectomy with the Solitaire $A B$ device in large artery occlusions of the anterior circulation: a pilot study. Stroke 41 (8): 1836-1840

Choulakian A, Alexander MJ (2010): Mechanical thrombectomy with the penumbra system for treatment of venous sinus thrombosis. J Neurolnterventional Surg 2(2): 153 
Clark WM, Wissman S, Albers GW, Jhamandas JH, Madden KP, Hamilton S (1999): Recombinant tissue-type plasminogen activator (Alteplase) for ischemic stroke 3 to 5 hours after symptom onset. The ATLANTIS Study: a randomized controlled trial. Alteplase Thrombolysis for Acute Noninterventional Therapy in Ischemic Stroke. JAMA 282(21): 2019-2026

Coutts SB, Goyal M (2009): When recanalization does not improve clinical outcomes. Stroke 40(8): 2661

del Zoppo GJ, Higashida RT, Furlan AJ, Pessin MS, Rowley HA, Gent M (1998): PROACT: a phase II randomized trial of recombinant pro-urokinase by direct arterial delivery in acute middle cerebral artery stroke. PROACT Investigators. Prolyse in Acute Cerebral Thromboembolism. Stroke 29(1): 4-11

Demchuk AM, Hill MD, Barber PA, Silver B, Patel SC, Levine SR (2005): Importance of early ischemic computed tomography changes using ASPECTS in NINDS rtPA Stroke Study. Stroke 36(10): 2110-2115

Devlin TG, Baxter BW, Feintuch TA, Desbiens NA (2007): The Merci Retrieval System for acute stroke: the Southeast Regional Stroke Center experience. Neurocrit Care $\underline{6}(1)$ : $11-21$

Dietl M, Pohle R, Weingartner M, Polgar R, Grassel E, Schwab S, Kolominsky-Rabas P (2009): Stroke etiology and long-term need of care in ischemic stroke patients. Fortschr Neurol Psychiatr $\underline{77}$ (12): 714-719

European Stroke Organisation (2008): Guidelines for management of ischaemic stroke and transient ischaemic attack 2008. Cerebrovasc Dis 25(5): 457-507

Feigin VL, Lawes CM, Bennett DA, Barker-Collo SL, Parag V (2009): Worldwide stroke incidence and early case fatality reported in 56 population-based studies: a systematic review. Lancet Neurol $\underline{8}(4): 355-369$

Fiebach JB, Schellinger PD, Jansen O, Meyer M, Wilde P, Bender J, Schramm P, Juttler E, Oehler J, Hartmann M, et al. (2002): CT and diffusion-weighted MR imaging in randomized order: diffusion-weighted imaging results in higher accuracy and lower interrater variability in the diagnosis of hyperacute ischemic stroke. Stroke $\underline{33}(9)$ : 2206-2210

Fiorelli M, Bastianello S, von Kummer R, del Zoppo GJ, Larrue V, Lesaffre E, Ringleb AP, Lorenzano S, Manelfe C, Bozzao L (1999): Hemorrhagic transformation within 36 hours of a cerebral infarct: relationships with early clinical deterioration and 3-month outcome in the European Cooperative Acute Stroke Study I (ECASS I) cohort. Stroke 30(11): 2280-2284

Fischer U, Arnold M, Nedeltchev K, Brekenfeld C, Ballinari P, Remonda L, Schroth G, Mattle HP (2005): NIHSS score and arteriographic findings in acute ischemic stroke. Stroke 36(10): 2121-2125

Flint AC, Cullen SP, Faigeles BS, Rao VA (2010): Predicting Long-Term Outcome after Endovascular Stroke Treatment: The Totaled Health Risks in Vascular Events Score. Am J Neuroradiol 31(7): 1192-1196

Furlan A, Higashida R, Wechsler L, Gent M, Rowley H, Kase C, Pessin M, Ahuja A, Callahan F, Clark WM, et al. (1999): Intra-arterial prourokinase for acute ischemic stroke. The 
PROACT II study: a randomized controlled trial. Prolyse in Acute Cerebral Thromboembolism. JAMA 282(21): 2003-2011

Giles MF, Rothwell PM (2010): Systematic review and pooled analysis of published and unpublished validations of the $A B C D$ and $A B C D 2$ transient ischemic attack risk scores. Stroke $\underline{41(4): 667-673}$

Gobin YP, Starkman S, Duckwiler GR, Grobelny T, Kidwell CS, Jahan R, Pile-Spellman J, Segal A, Vinuela F, Saver JL (2004): MERCI 1: a phase 1 study of Mechanical Embolus Removal in Cerebral Ischemia. Stroke 35(12): 2848-2854

Goldstein LB, Bertels C, Davis JN (1989): Interrater reliability of the NIH stroke scale. Arch Neurol 46(6): 660-662

Gonzalez RG, Hirsch JA, Lev MH, Schaefer PW, Schwamm LH: Acute Ischemic Stroke: Imaging and Intervention. 2nd Edition. Springer, Berlin 2010

Goyal M, Menon BK, Coutts SB, Hill MD, Demchuk AM (2011): Effect of baseline CT scan appearance and time to recanalization on clinical outcomes in endovascular thrombectomy of acute ischemic strokes. Stroke $\underline{42(1): ~ 93-97}$

Gralla J, Brekenfeld C, Mordasini P, Schroth G (2012): Mechanical thrombolysis and stenting in acute ischemic stroke. Stroke $\underline{43}(1): 280-285$

Granger CV, Dewis LS, Peters NC, Sherwood CC, Barrett JE (1979): Stroke rehabilitation: analysis of repeated Barthel index measures. Arch Phys Med Rehabil $\underline{60}(1)$ : 14-17

Grant PE, He J, Halpern EF, Wu O, Schaefer PW, Schwamm LH, Budzik RF, Sorensen AG, Koroshetz WJ, Gonzalez RG (2001): Frequency and clinical context of decreased apparent diffusion coefficient reversal in the human brain. Radiology 221(1): 43-50

Grau AJ, Weimar C, Buggle F, Heinrich A, Goertler M, Neumaier S, Glahn J, Brandt T, Hacke W, Diener HC (2001): Risk factors, outcome, and treatment in subtypes of ischemic stroke: the German stroke data bank. Stroke 32(11): 2559-2566

Grau AJ, Eicke M, Biegler MK, Faldum A, Bamberg C, Haass A, Hardt R, Hufschmidt A, Lowitzsch K, Marx J, et al. (2010): Quality monitoring of acute stroke care in Rhineland-Palatinate, Germany, 2001-2006. Stroke 41(7): 1495-1500

Grunwald IQ, Walter S, Papanagiotou P, Krick C, Hartmann K, Dautermann A, Fassbender K, Haass A, Bolar LJ, Reith W, et al. (2009): Revascularization in acute ischaemic stroke using the penumbra system: the first single center experience. Eur $\mathrm{J}$ Neurol 16(11): 1210-1216

Gupta R (2009): Arterial vasospasm during mechanical thrombectomy for acute stroke. J Neuroimaging 19(1): 61-64

Hacke W, Kaste M, Fieschi C, Toni D, Lesaffre E, von Kummer R, Boysen G, Bluhmki E, Hoxter G, Mahagne $\mathrm{MH}$, et al. (1995): Intravenous thrombolysis with recombinant tissue plasminogen activator for acute hemispheric stroke. The European Cooperative Acute Stroke Study (ECASS). JAMA 274(13): 1017-1025

Hacke W, Kaste M, Fieschi C, von Kummer R, Davalos A, Meier D, Larrue V, Bluhmki E, Davis S, Donnan G, et al. (1998): Randomised double-blind placebo-controlled trial of thrombolytic therapy with intravenous alteplase in acute ischaemic stroke (ECASS II). 
Second European-Australasian Acute Stroke Study Investigators. Lancet 352(9136): $1245-1251$

Hacke W, Donnan G, Fieschi C, Kaste M, von Kummer R, Broderick JP, Brott T, Frankel M, Grotta JC, Haley EC, et al. (2004): Association of outcome with early stroke treatment: pooled analysis of ATLANTIS, ECASS, and NINDS rt-PA stroke trials. Lancet 363(9411): 768-774

Hacke W, Kaste M, Bluhmki E, Brozman M, Davalos A, Guidetti D, Larrue V, Lees KR, Medeghri Z, Machnig T, et al. (2008): Thrombolysis with alteplase 3 to 4.5 hours after acute ischemic stroke. N Engl J Med 359(13): 1317-1329

Harjung C: Intrakranielle Angioplastie und Stentimplantation bei symptomatischen Patienten: Erfolgsraten, Komplikationsraten und Langzeitergebnisse des Kollektivs der Universitätsmedizin Göttingen. Med. Diss. Göttingen 2009

Heinemann AW, Harvey RL, McGuire JR, Ingberman D, Lovell L, Semik P, Roth EJ (1997): Measurement properties of the NIH Stroke Scale during acute rehabilitation. Stroke 28(6): $1174-1180$

Heiss WD, Podreka I (1993): Role of PET and SPECT in the assessment of ischemic cerebrovascular disease. Cerebrovasc Brain Metab Rev $\underline{5}$ (4): 235-263

Henkes H, Reinartz J, Lowens S, Miloslavski E, Roth C, Reith W, Kuhne D (2006): A device for fast mechanical clot retrieval from intracranial arteries (Phenox clot retriever). Neurocrit Care $\underline{5}(2): 134-140$

Higashida RT, Furlan AJ, Roberts H, Tomsick T, Connors B, Barr J, Dillon W, Warach S, Broderick J, Tilley B, et al. (2003): Trial design and reporting standards for intraarterial cerebral thrombolysis for acute ischemic stroke. Stroke 34(8): 109-137

Hjort N, Butcher K, Davis SM, Kidwell CS, Koroshetz WJ, Rother J, Schellinger PD, Warach S, Ostergaard L (2005): Magnetic resonance imaging criteria for thrombolysis in acute cerebral infarct. Stroke $\underline{36}(2): 388-397$

Howard G, McClure LA, Krakauer JW, Coffey CS (2007): Stroke and the statistics of the aspirin/clopidogrel secondary prevention trials. Curr Opin Neurol 20(1): 71-77

Huded V, Saraf R, Limaye U (2009): Mechanical device the Penumbra system in the management of acute stroke: Report of five cases. Neurol India $\underline{57(3): 310-312}$

Hui FK, Hussain MS, Spiotta A, Bhalla T, Toth G, Moskowitz SI, Elgabaly M, Sivapatham T, Rasmussen PA (2011): Merci Retrievers as Access Adjuncts for Reperfusion Catheters: The Grappling Hook Technique. Neurosurgery $\underline{70}$ (2): 456-460

IMS Study Group (2004): Combined intravenous and intra-arterial recanalization for acute ischemic stroke: the Interventional Management of Stroke Study. Stroke 35(4): 904911

IMS II Trial Investigators (2007): The Interventional Management of Stroke (IMS) II Study. Stroke $\underline{38}(7): 2127-2135$

Ishkanian AA, McCullough-Hicks ME, Appelboom G, Piazza MA, Hwang BY, Bruce SS, Hannan LM, Connolly ES, Lavine SD, Meyers PM (2011): Improving patient selection for endovascular treatment of acute cerebral ischemia: a review of the literature and 
an external validation of the Houston IAT and THRIVE predictive scoring systems. Neurosurg Focus $\underline{30}(6)$ : E7

Jennett B, Bond M (1975): Assessment of outcome after severe brain damage. Lancet 1975, I, $480-484$

Johnson MB, Wilkinson ID, Wattam J, Venables GS, Griffiths PD (2000): Comparison of Doppler ultrasound, magnetic resonance angiographic techniques and catheter angiography in evaluation of carotid stenosis. Clin Radiol 55(12): 912-920

Johnston SC, Gress DR, Browner WS, Sidney S (2000): Short-term prognosis after emergency department diagnosis of TIA. JAMA 284(22): 2901

Johnston SC, Rothwell PM, Nguyen-Huynh MN, Giles MF, Elkins JS, Bernstein AL, Sidney S (2007): Validation and refinement of scores to predict very early stroke risk after transient ischaemic attack. Lancet $\underline{369}$ (9558): 283-292

Josephson SA, Hills NK, Johnston SC (2006): NIH Stroke Scale reliability in ratings from a large sample of clinicians. Cerebrovasc Dis 22(5-6): 389-395

Kang DH, Kim YS, Park J, Hwang YH (2011): Rescue forced suction thrombectomy using the reperfusion catheter of the Penumbra System for thromboembolism during coil embolization of ruptured cerebral aneurysms. Neurosurgery $\underline{70}(1)$ : 89-93

Kasner SE, Chalela JA, Luciano JM, Cucchiara BL, Raps EC, McGarvey ML, Conroy MB, Localio AR (1999): Reliability and validity of estimating the NIH stroke scale score from medical records. Stroke 30(8): 1534-1537

Kaufmann TJ, Huston J, Mandrekar JN, Schleck CD, Thielen KR, Kallmes DF (2007): Complications of diagnostic cerebral angiography: evaluation of 19,826 consecutive patients. Radiology 243(3): 812-819

Khatri P, Hill MD, Palesch YY, Spilker J, Jauch EC, Carrozzella JA, Demchuk AM, Martin R, Mauldin P, Dillon C, et al. (2008): Methodology of the Interventional Management of Stroke III Trial. Stroke $\underline{3}(2): 130-137$

Khatri P, Abruzzo T, Yeatts SD, Nichols C, Broderick JP, Tomsick TA (2009): Good clinical outcome after ischemic stroke with successful revascularization is time-dependent. Neurology 73(13): 1066-1072

Kim AS, Nguyen-Huynh M, Johnston SC (2011): A cost-utility analysis of mechanical thrombectomy as an adjunct to intravenous tissue-type plasminogen activator for acute large-vessel ischemic stroke. Stroke 42(7): 2013-2018

Kim D, Jahan R, Starkman S, Abolian A, Kidwell CS, Vinuela F, Duckwiler GR, Ovbiagele B, Vespa PM, Selco S, et al. (2006): Endovascular mechanical clot retrieval in a broad ischemic stroke cohort. Am J Neuroradiol 27(10): 2048-2052

Knauth M, von Kummer R, Jansen O, Hahnel S, Dorfler A, Sartor K (1997): Potential of CT angiography in acute ischemic stroke. Am J Neuroradiol 18(6): 1001-1010

Koenig M, Kraus M, Theek C, Klotz E, Gehlen W, Heuser L (2001): Quantitative assessment of the ischemic brain by means of perfusion-related parameters derived from perfusion CT. Stroke 32(2): 431-437 
Köhrmann M, Juttler E, Fiebach JB, Huttner HB, Siebert S, Schwark C, Ringleb PA, Schellinger PD, Hacke W (2006): MRI versus CT-based thrombolysis treatment within and beyond the $3 \mathrm{~h}$ time window after stroke onset: a cohort study. Lancet Neurol ㄱ(8): $661-667$

Köhrmann M (2010): Prähospitalversorgung und Akuttherapie des ischämischen Schlaganfalls. Intensivmed Notfallmed $\underline{47}(3)$ : 149-155

Kolominsky-Rabas PL, Heuschmann PU (2002): Incidence, etiology and long-term prognosis of stroke. Fortschr Neurol Psychiatr $\underline{70}$ (12): 657-662

Kolominsky-Rabas PL, Heuschmann PU, Marschall D, Emmert M, Baltzer N, Neundorfer B, Schoffski, Krobot KJ (2006): Lifetime cost of ischemic stroke in Germany: results and national projections from a population-based stroke registry: the Erlangen Stroke Project. Stroke $\underline{37(5): 1179-1183}$

Kreusch A, Knauth M: Aspirationssysteme; in: Interventionelle Therapie des Schlaganfalls; Jansen O. und Brückmann H.; Georg Thieme Verlag, Stuttgart 2011, 86-94

Kreusch AS, Psychogios MN, Knauth M (2012): Techniques and results - penumbra aspiration catheter. Tech Vasc Interv Radiol 15(1): 53-59

Kulcsar Z, Bonvin C, Pereira VM, Altrichter S, Yilmaz H, Lovblad KO, Sztajzel R, Rufenacht DA (2010 a): Penumbra System: A Novel Mechanical Thrombectomy Device for Large-Vessel Occlusions in Acute Stroke. Am J Neuroradiol 31(4): 628-633

Kulcsar Z, Marosfoi M, Berentei Z, Szikora I (2010 b): Continuous thrombolysis and repeated thrombectomy with the Penumbra System in a child with hemorrhagic sinus thrombosis: technical note. Acta Neurochir (Wien) 152(5): 911-916

Latchaw RE (2004): Cerebral perfusion imaging in acute stroke. J Vasc Interv Radiol 15(1): $29-46$

Latchaw RE, Alberts MJ, Lev MH, Connors JJ, Harbaugh RE, Higashida RT, Hobson R, Kidwell CS, Koroshetz WJ, Mathews V, et al. (2009): Recommendations for imaging of acute ischemic stroke: a scientific statement from the American Heart Association. Stroke $\underline{40}$ (11): 3646-3678

Lees KR, Bluhmki E, von Kummer R, Brott TG, Toni D, Grotta JC, Albers GW, Kaste M, Marler JR, Hamilton SA, et al. (2010): Time to treatment with intravenous alteplase and outcome in stroke: an updated pooled analysis of ECASS, ATLANTIS, NINDS, and EPITHET trials. Lancet $\underline{375}$ (9727): 1695-1703

Leker RR, Liebeskind DS (2009): Penumbra and reperfusion in acute ischaemic stroke: what's in a name? Eur J Neurol 16(11): 1169-1170

Lev MH, Farkas J, Rodriguez VR, Schwamm LH, Hunter GJ, Putman CM, Rordorf GA, Buonanno FS, Budzik R, Koroshetz WJ, et al. (2001): CT angiography in the rapid triage of patients with hyperacute stroke to intraarterial thrombolysis: accuracy in the detection of large vessel thrombus. J Comput Assist Tomogr 25(4): 520-528

Levy EI, Siddiqui AH, Crumlish A, Snyder KV, Hauck EF, Fiorella DJ, Hopkins LN, Mocco J (2009): First Food and Drug Administration-approved prospective trial of primary intracranial stenting for acute stroke: SARIS (stent-assisted recanalization in acute ischemic stroke). Stroke $\underline{40}$ (11): 3552-3556 
Lindsberg PJ, Mattle HP (2006): Therapy of basilar artery occlusion: a systematic analysis comparing intra-arterial and intravenous thrombolysis. Stroke $\underline{37(3): 922-928}$

Lisboa RC, Jovanovic BD, Alberts MJ (2002): Analysis of the safety and efficacy of intraarterial thrombolytic therapy in ischemic stroke. Stroke 33(12): 2866-2871

Lloyd-Jones D, Adams RJ, Brown TM, Carnethon M, Dai S, De Simone G, Ferguson TB, Ford E, Furie K, Gillespie C, et al. (2010): Heart disease and stroke statistics - 2010 update: a report from the American Heart Association. Circulation 121(7): 948-954

Machi P, Costalat V, Lobotesis K, Lima Maldonado I, Vendrell JF, Riquelme C, Bonafe A (2011): Solitaire FR thrombectomy system: immediate results in 56 consecutive acute ischemic stroke patients. J Neurointerv Surg $\underline{4}(1)$ : 62-66

Macleod MR, Davis SM, Mitchell PJ, Gerraty RP, Fitt G, Hankey GJ, Stewart-Wynne EG, Rosen D, McNeil JJ, Bladin CF, et al. (2005): Results of a multicentre, randomised controlled trial of intra-arterial urokinase in the treatment of acute posterior circulation ischaemic stroke. Cerebrovasc Dis 20(1): 12-17

Mahoney FI, Barthel DW (1965): Functional Evaluation: The Barthel Index. Md State Med J 14: $61-65$

Marks MP, Holmgren EB, Fox AJ, Patel S, von Kummer R, Froehlich J (1999): Evaluation of early computed tomographic findings in acute ischemic stroke. Stroke $\underline{30}(2): 389-392$

Mattle HP, Arnold M, Georgiadis D, Baumann C, Nedeltchev K, Benninger D, Remonda L, von Büdingen C, Diana A, Pangalu A, et al. (2008): Comparison of intraarterial and intravenous thrombolysis for ischemic stroke with hyperdense middle cerebral artery sign. Stroke $\underline{39}(2)$ : 379-383

Mazighi M, Serfaty JM, Labreuche J, Laissy JP, Meseguer E, Lavallee PC, Cabrejo L, Slaoui T, Guidoux C, Lapergue B, et al. (2009): Comparison of intravenous alteplase with a combined intravenous-endovascular approach in patients with stroke and confirmed arterial occlusion (RECANALISE study): a prospective cohort study. Lancet Neurol 8(9): 802-809

Menon BK, Hill MD, Eesa M, Modi J, Bhatia R, Wong J, Hudon ME, Morrish W, Demchuk A M, Goyal M (2010): Initial experience with the Penumbra Stroke System for recanalization of large vessel occlusions in acute ischemic stroke. Neuroradiology $\underline{53}(4): 261-266$

Meyers PM, Schumacher HC, Connolly ES, Heyer EJ, Gray WA, Higashida RT (2011): Current status of endovascular stroke treatment. Circulation 123(22): 2591-2601

Molina CA, Ribo M, Rubiera M, Montaner J, Santamarina E, Delgado-Mederos R, Arenillas JF, Huertas R, Purroy F, Delgado P, et al. (2006): Microbubble administration accelerates clot lysis during continuous $2-\mathrm{MHz}$ ultrasound monitoring in stroke patients treated with intravenous tissue plasminogen activator. Stroke $\underline{37}(2)$ : $425-429$

Moseley ME, Kucharczyk J, Mintorovitch J, Cohen Y, Kurhanewicz J, Derugin N, Asgari H, Norman D (1990): Diffusion-weighted MR imaging of acute stroke: correlation with T2-weighted and magnetic susceptibility-enhanced MR imaging in cats. Am J Neuroradiol 11(3): 423-429 
Mourand I, Brunel H, Costalat V, Riquelme C, Lobotesis K, Milhaud D, Heroum C, Arquizan C, Moynier M, Bonafe A (2011): Mechanical thrombectomy in acute ischemic stroke: catch device. Am J Neuroradiol 32(8): 1381-1385

Muir KW, Buchan A, von Kummer R, Rother J, Baron JC (2006): Imaging of acute stroke. Lancet Neurol $\underline{5}(9): 755-768$

Muir KW, Baird-Gunning J, Walker L, Baird T, McCormick M, Coutts SB (2007): Can the ischemic penumbra be identified on noncontrast CT of acute stroke? Stroke $\underline{38}(9)$ : $2485-2490$

Nguyen TN, Babikian VL, Romero R, Pikula A, Kase CS, Jovin TG, Norbash AM (2011): Intra-arterial treatment methods in acute stroke therapy. Front Neurol 2: 9

Nguyen-Huynh MN, Johnston SC (2011): Is mechanical clot removal or disruption a costeffective treatment for acute stroke? Am J Neuroradiol 32(2): 244-249

NINDS rt-PA Stroke Study Group (1995): Tissue plasminogen activator for acute ischemic stroke. The National Institute of Neurological Disorders and Stroke rt-PA Stroke Study Group. N Engl J Med 333(24): 1581-1587

NINDS t-PA Stroke Study Group (1997): Intracerebral hemorrhage after intravenous t-PA therapy for ischemic stroke. The NINDS t-PA Stroke Study Group. Stroke 28(11): $2109-2118$

Nogueira RG, Liebeskind DS, Sung G, Duckwiler G, Smith WS (2009): Predictors of good clinical outcomes, mortality, and successful revascularization in patients with acute ischemic stroke undergoing thrombectomy: pooled analysis of the Mechanical Embolus Removal in Cerebral Ischemia (MERCI) and Multi MERCI Trials. Stroke 40(12): 3777-3783

Nogueira RG, Levy El, Gounis M, Siddiqui AH (2011): The Trevo device: preclinical data of a novel stroke thrombectomy device in two different animal models of arterial thromboocclusive disease. J Neurointerv Surg 4(4): 295-300

Ogawa A, Mori E, Minematsu K, Taki W, Takahashi A, Nemoto S, Miyamoto S, Sasaki M, Inoue T. (2007): Randomized trial of intraarterial infusion of urokinase within 6 hours of middle cerebral artery stroke: the middle cerebral artery embolism local fibrinolytic intervention trial (MELT) Japan. Stroke 38(10): 2633-2639

Ostergaard L (2005): Principles of cerebral perfusion imaging by bolus tracking. J Magn Reson Imaging 22(6): 710-717

Paciaroni M, Caso V, Agnelli G (2009): The concept of ischemic penumbra in acute stroke and therapeutic opportunities. Eur Neurol 61(6): 321-330

Patil CG, Long EF, Lansberg MG (2009): Cost-effectiveness analysis of mechanical thrombectomy in acute ischemic stroke. J Neurosurg 110(3): 508-513

Penumbra Pivotal Stroke Trial Investigators (2009): The penumbra pivotal stroke trial: safety and effectiveness of a new generation of mechanical devices for clot removal in intracranial large vessel occlusive disease. Stroke $\underline{40}$ (8): 2761-2768

Petty GW, Brown RD, Whisnant JP, Sicks JD, O'Fallon WM, Wiebers DO (1999): Ischemic stroke subtypes: a population-based study of incidence and risk factors. Stroke 30(12): 2513-2516 
Pexman JH, Barber PA, Hill MD, Sevick RJ, Demchuk AM, Hudon ME, Hu WY, Buchan AM (2001): Use of the Alberta Stroke Program Early CT Score (ASPECTS) for assessing CT scans in patients with acute stroke. Am J Neuroradiol 22(8): 1534-1542

Powers WJ, Grubb RL, Darriet D, Raichle ME (1985): Cerebral blood flow and cerebral metabolic rate of oxygen requirements for cerebral function and viability in humans. $J$ Cereb Blood Flow Metab $\underline{5}(4)$ : 600-608

Psychogios MN, Kreusch A, Wasser K, Mohr A, Groschel K, Knauth M (2012): Recanalization of Large Intracranial Vessels Using the Penumbra System: A Single Center Experience. (Am J Neuroradiol, im Druck)

Quinn TJ, Dawson J, Walters M (2008): Dr John Rankin; his life, legacy and the 50th anniversary of the Rankin Stroke Scale. Scott Med J 53(1): 44-47

Quinn TJ, Dawson J, Walters MR, Lees KR (2009 a): Exploring the reliability of the modified rankin scale. Stroke $\underline{40}(3): 762-766$

Quinn TJ, Paolucci S, Sivenius J, Walker MF, Toni D, Lees KR (2009 b): Evidence-based stroke rehabilitation: an expanded guidance document from the european stroke organisation (ESO) guidelines for management of ischaemic stroke and transient ischaemic attack 2008. J Rehabil Med 41(2): 99-111

Qureshi AI, Ali Z, Suri MF, Kim SH, Shatla AA, Ringer AJ, Lopes DK, Guterman LR, Hopkins LN (2001): Intra-arterial third-generation recombinant tissue plasminogen activator (reteplase) for acute ischemic stroke. Neurosurgery 49(1): 41-48

Qureshi Al, Suarez JI, Yahia AM, Mohammad Y, Uzun G, Suri MF, Zaidat OO, Ayata C, Ali Z, Wityk RJ (2003): Timing of neurologic deterioration in massive middle cerebral artery infarction: a multicenter review. Crit Care Med 31(1): 272-277

Rankin J (1957): Cerebral vascular accidents in patients over the age of 60. II. Prognosis. Scott Med J 2 (5): 200-215

Rha JH, Saver JL (2007): The impact of recanalization on ischemic stroke outcome: a metaanalysis. Stroke $\underline{38(3): ~ 967-973}$

Riedel CH, Zimmermann P, Jensen-Kondering U, Stingele R, Deuschl G, Jansen O (2011): The importance of size: successful recanalization by intravenous thrombolysis in acute anterior stroke depends on thrombus length. Stroke $\underline{42(6): 1775-1777}$

Ringleb P, Schellinger PD, Hacke W (2008): European Stroke Organisation 2008 guidelines for managing acute cerebral infarction or transient ischemic attack. Part 1. Nervenarzt 79(8): 936-957

Rohde S, Haehnel S, Herweh C, Pham M, Stampfl S, Ringleb PA, Bendszus M (2011): Mechanical thrombectomy in acute embolic stroke: preliminary results with the revive device. Stroke 42(10): 2954-2956

Rorick MB, Nichols FT, Adams RJ (1994): Transcranial Doppler correlation with angiography in detection of intracranial stenosis. Stroke 25(10): 1931-1934

Sacco RL (1997): Risk factors, outcomes, and stroke subtypes for ischemic stroke. Neurology $\underline{49}$ (5 Suppl 4): S39-44 
Saur D, Kucinski T, Grzyska U, Eckert B, Eggers C, Niesen W, Schoder V, Zeumer H, Weiller C, Rother J. (2003): Sensitivity and interrater agreement of CT and diffusionweighted MR imaging in hyperacute stroke. Am J Neuroradiol 24(5): 878-885

Saver JL (2006): Time is brain - quantified. Stroke $\underline{37(1): 263-266}$

Saver JL (2011): Improving reperfusion therapy for acute ischaemic stroke. J Thromb Haemost $\underline{9}$ (Suppl 1): 333-343

Saver JL, Albers GW, Dunn B, Johnston KC, Fisher M (2009): Stroke Therapy Academic Industry Roundtable (STAIR) recommendations for extended window acute stroke therapy trials. Stroke $\underline{40}(7): 2594-2600$

Saver JL, Liebeskind DS, Nogueira RG, Jahan R (2010): Need to clarify Thrombolysis In Myocardial Ischemia (TIMI) scale scoring method in the Penumbra Pivotal Stroke Trial. Stroke 41(2): 115-116

Schellinger PD, Hacke W (2010): Recanalization devices should be restricted to clinical trials: pro (kind of). Stroke 41(1): 191-193

Schellinger PD, Thomalla G, Fiehler J, Köhrmann M, Molina CA, Neumann-Haefelin T, Ribo M, Singer OC, Zaro-Weber O, Sobesky J (2007): MRI-based and CT-based thrombolytic therapy in acute stroke within and beyond established time windows: an analysis of 1210 patients. Stroke $\underline{38}(10): 2640-2645$

Schlaug G, Benfield A, Baird AE, Siewert B, Lovblad KO, Parker RA, Edelman RR, Warach $S$ (1999): The ischemic penumbra: operationally defined by diffusion and perfusion MRI. Neurology $\underline{53}(7): 1528-1537$

Schmulling S, Grond M, Rudolf J, Kiencke P (1998): Training as a prerequisite for reliable use of NIH Stroke Scale. Stroke 29(6): 1258-1259

Schramm P, Schellinger PD, Klotz E, Kallenberg K, Fiebach JB, Kulkens S, Heiland S, Knauth M, Sartor K (2004): Comparison of perfusion computed tomography and computed tomography angiography source images with perfusion-weighted imaging and diffusion-weighted imaging in patients with acute stroke of less than 6 hours' duration. Stroke $\underline{35}(7): 1652-1658$

Serna-Candel C, Lopez-lbor L, Matias-Guiu J (2010): Endovascular treatment for acute stroke: an open field to begin. Neurologia 25(5): 273-278

Shaltoni HM, Albright KC, Gonzales NR, Weir RU, Khaja AM, Sugg RM, Campbell MS, Cacayorin ED, Grotta JC, Noser EA (2007): Is intra-arterial thrombolysis safe after full-dose intravenous recombinant tissue plasminogen activator for acute ischemic stroke? Stroke 38(1): 80-84

Sheehan FH, Braunwald E, Canner P, Dodge HT, Gore J, Van Natta P, Passamani ER, Williams DO, Zaret B (1987): The effect of intravenous thrombolytic therapy on left ventricular function: a report on tissue-type plasminogen activator and streptokinase from the Thrombolysis in Myocardial Infarction (TIMI Phase I) trial. Circulation 75(4): 817-829

Siegenthaler W, Blum HE: Klinische Pathophysiologie. 9. Auflage. Georg Thieme Verlag, Stuttgart 2006 
Smith WS, Sung G, Starkman S, Saver JL, Kidwell CS, Gobin YP, Lutsep HL, Nesbit GM, Grobelny T, Rymer MM, et al. (2005): Safety and efficacy of mechanical embolectomy in acute ischemic stroke: results of the MERCI trial. Stroke $\underline{36}(7)$ : 14321438

Smith WS, Sung G, Saver J, Budzik R, Duckwiler G, Liebeskind DS, Lutsep HL, Rymer MM, Higashida RT, Starkman S, et al. (2008): Mechanical thrombectomy for acute ischemic stroke: final results of the Multi MERCI trial. Stroke 39(4): 1205-1212

Soares BP, Chien JD, Wintermark M (2009): MR and CT monitoring of recanalization, reperfusion, and penumbra salvage: everything that recanalizes does not necessarily reperfuse! Stroke $\underline{40}$ (3 Suppl): 24-27

Spilker J, Kongable G, Barch C, Braimah J, Brattina P, Daley S, Donnarumma R, Rapp K, Sailor S (1997): Using the NIH Stroke Scale to assess stroke patients. The NINDS rtPA Stroke Study Group. J Neurosci Nurs 29 (6): 384-392

Stroke Unit Trialists' Collaboration (2007): Organised inpatient (stroke unit) care for stroke. Cochrane Database Syst Rev (4): CD000197

Struffert T, Köhrmann M, Engelhorn T, Nowe T, Richter G, Schellinger P, Schwab S, Doerfler A (2009): Penumbra Stroke System as an "add-on" for the treatment of large vessel occlusive disease following thrombolysis: first results. Eur Radiol 19(9): 2286-2293

Takasawa M, Jones PS, Guadagno JV, Christensen S, Fryer TD, Harding S, Gillard JH, Williams GB, Aigbirhio FI, Warburton EA, et al. (2008): How reliable is perfusion MR in acute stroke? Validation and determination of the penumbra threshold against quantitative PET. Stroke $\underline{39}$ (3): 870-877

Tarr R, Hsu D, Kulcsar Z, Bonvin C, Rufenacht D, Alfke K, Stingele R, Jansen O, Frei D, Bellon R, et al. (2010): The POST trial: initial post-market experience of the Penumbra system: revascularization of large vessel occlusion in acute ischemic stroke in the United States and Europe. J Neurointerv Surg 2(4): 341-344

Thomalla G, Audebert HJ, Berger K, Fiebach JB, Fiehler J, Kaps M, Neumann-Haefelin T, Schellinger PD, Siebler M, Sobesky J, et al. (2009): Bildgebung beim Schlaganfall eine Übersicht und Empfehlungen des Kompetenznetzes Schlaganfall. Akt Neurol 36(07): 354-367

Thomalla G, Cheng B, Ebinger M, Hao Q, Tourdias T, Wu O, Kim JS, Breuer L, Singer OC, Warach S, et al. (2011): DWI-FLAIR mismatch for the identification of patients with acute ischaemic stroke within $4.5 \mathrm{~h}$ of symptom onset (PRE-FLAIR): a multicentre observational study. Lancet Neurol 10(11): 978-986

TIMI Study Group (1985): The Thrombolysis in Myocardial Infarction (TIMI) trial. Phase I findings. TIMI Study Group. N Engl J Med 312(14): 932-936

Tomsick T (2007): TIMI, TIBI, TICI: I came, I saw, I got confused. Am J Neuroradiol 28(2): 382-384

Tong DC, Yenari MA, Albers GW, O'Brien M, Marks MP, Moseley ME (1998): Correlation of perfusion- and diffusion-weighted MRI with NIHSS score in acute ( $<6.5$ hour) ischemic stroke. Neurology 50(4): 864-870

Trenkler J (2008): Der akute ischämische Schlaganfall. Radiologe $\underline{48(5): ~ 457-473}$ 
Vahedi K, Hofmeijer J, Juettler E, Vicaut E, George B, Algra A, Amelink GJ, Schmiedeck P, Schwab S, Rothwell PM, et al. (2007): Early decompressive surgery in malignant infarction of the middle cerebral artery: a pooled analysis of three randomised controlled trials. Lancet Neurol $\underline{6}(3): 215-222$

Villablanca JP, Martin N, Jahan R, Gobin YP, Frazee, Duckwiler G, Bentson J, Hardart M, Coiteiro D, Sayre J, et al. (2000): Volume-rendered helical computerized tomography angiography in the detection and characterization of intracranial aneurysms. J Neurosurg 93(2): 254-264

von Kummer R, Allen KL, Holle R, Bozzao L, Bastianello S, Manelfe C, Bluhmki E, Ringleb P, Meier DH, Hacke W (1997): Acute stroke: usefulness of early CT findings before thrombolytic therapy. Radiology 205(2): 327

Wahlgren N, Ahmed N, Davalos A, Ford GA, Grond M, Hacke W, Hennerici MG, Kaste M, Kuelkens S, Larrue V, et al. (2007): Thrombolysis with alteplase for acute ischaemic stroke in the Safe Implementation of Thrombolysis in Stroke-Monitoring Study (SITSMOST): an observational study. Lancet $\underline{369}$ (9558): 275-282

Wardlaw JM, Mielke O (2005): Early Signs of Brain Infarction at CT: Observer Reliability and Outcome after Thrombolytic Treatment - Systematic Review. Radiology 235(2): 444

Wardlaw JM, Murray V, Berge E, Del Zoppo GJ (2009): Thrombolysis for acute ischaemic stroke. Cochrane Database Syst Rev (4): CD000213

Waxman SG, Toole JF (1983): Temporal profile resembling TIA in the setting of cerebral infarction. Stroke 14(3): 433

WHO: The global burden of disease: 2004 update. WHO Press, Geneva 2008

Williams LS, Yilmaz EY, Lopez-Yunez AM (2000): Retrospective assessment of initial stroke severity with the NIH Stroke Scale. Stroke 31(4): 858-862

Willinsky RA, Taylor SM, TerBrugge K, Farb RI, Tomlinson G, Montanera W (2003): Neurologic complications of cerebral angiography: prospective analysis of 2,899 procedures and review of the literature. Radiology 227(2): 522-528

Wintermark M, Reichhart $M$, Cuisenaire $O$, Maeder $P$, Thiran JP, Schnyder $P$, Bogousslavsky J, Meuli R (2002): Comparison of admission perfusion computed tomography and qualitative diffusion- and perfusion-weighted magnetic resonance imaging in acute stroke patients. Stroke 33(8): 2025-2031

Wintermark M, Meuli R, Browaeys P, Reichhart M, Bogousslavsky J, Schnyder P, Michel P (2007): Comparison of CT perfusion and angiography and MRI in selecting stroke patients for acute treatment. Neurology $\underline{68(9): 694-697}$

Yuki I, Kan I, Vinters HV, Kim RH, Golshan A, Vinuela FA, Sayre JW, Murayama Y, Vinuela F (2011): The Impact of Thromboemboli Histology on the Performance of a Mechanical Thrombectomy Device. Am J Neuroradiol 33(4): 643-648

Zeumer H, Hacke W, Kolmann HL, Poeck K (1982): Lokale Fibrinolysetherapie bei BasilarisThrombose. Dtsch Med Wochenschr 107(19): 728-731 


\section{Wissenschaftliche Veröffentlichungen zum Thema der Dissertation}

\section{Originalarbeiten}

Kreusch AS, Psychogios MN, Knauth M (2012): Techniques and results - penumbra aspiration catheter. Tech Vasc Interv Radiol 15(1):53-59

Psychogios MN, Kreusch A, Wasser K, Mohr A, Groschel K, Knauth M (2012): Recanalization of Large Intracranial Vessels Using the Penumbra System: A Single Center Experience. AJNR Am J Neuroradiol 33(8):1488-93

\section{Buchbeiträge}

Kreusch A, Knauth M: Aspirationssysteme; In: Interventionelle Therapie des Schlaganfalls; Jansen O. und Brückmann H.; Georg Thieme Verlag, Stuttgart 2011, 86-94

\section{Kongressbeiträge}

Kreusch A, Psychogios MN, Wasser K, Knauth M: Mechanische Rekanalisation bei akutem ischämischen Schlaganfall unter Verwendung des Penumbra Systems erste Ergebnisse. Vortrag am 24. Oktober 2009 in Berlin. 5. Penumbra User Meeting.

Kreusch A, Psychogios MN, Wasser K, Knauth M: Mechanische Rekanalisation bei akutem ischämischen Schlaganfall unter Verwendung des Penumbra Systems - eine 2-Jahres-Evaluation von 86 Patienten. Vortrag am 13. Mai 2010 in Berlin. 91. Deutscher Röntgenkongress der Deutschen Röntgengesellschaft e.V., 12. - 15. Mai 2010.

Kreusch A: Promotion in der Radiologie - ein Erfahrungsbericht. Vortrag am 15. Mai 2010 in Berlin. Stipendienprogramm „Die hellsten Köpfe für die Radiologie“ der 
Deutschen Röntgengesellschaft e.V. auf dem 91. Deutschen Röntgenkongress, 12. 15. Mai 2010.

Psychogios MN, Kreusch A, Wasser K, Knauth M: Penumbra System: A New Mechanical Thrombectomy Device for Arterial Occlusions in Acute Stroke. Vortrag am 29. November 2010 in Chicago / USA. 96th Scientific Assembly and Annual Meeting der Radiological Society of North America (RSNA), 28. November - 3. Dezember 2010.

Kreusch A, Psychogios MN, Wasser K, Knauth M: Mechanische Rekanalisation bei akutem ischämischen Schlaganfall unter Verwendung des Penumbra Systems - eine Evaluation von 91 Patienten. Vortrag am 7. Oktober 2011 in Köln. 46. Jahrestagung der Deutschen Gesellschaft für Neuroradiologie, 6. - 8. Oktober 2011. 


\section{Danksagung}

Zum Abschluss möchte ich allen herzlich danken, die zum Gelingen dieser Arbeit beigetragen haben.

Zuallererst danke ich meinem Doktorvater Herrn Prof. Dr. med. Michael Knauth für die Bereitstellung des spannenden Themas in der Abteilung für Neuroradiologie und das Heranführen an das wissenschaftliche Arbeiten. Die sehr gute und freundliche Betreuung habe ich sehr geschätzt und er hat es geschafft, meinen Enthusiasmus für dieses Fachgebiet zu wecken. Weiterhin möchte ich mich bei inm für die außergewöhnliche Unterstützung, Förderung, weit über die Promotion hinaus, bei Kongressteilnahmen, wissenschaftlichen Publikationen und Auslandsaufenthalten sowie für das entgegengebrachte Vertrauen bedanken.

Ganz besonderer Dank gilt auch meinem Betreuer Marios Psychogios der Abteilung Neuroradiologie, der mich während der gesamten Zeit sehr unterstützt hat. Er hatte immer ein offenes Ohr für aufkommende Fragen und war eine große Hilfe bei der Auswertung der Daten und Korrektur der schriftlichen Ausarbeitung meiner Dissertation.

Ein großer Dank gilt ebenfalls meiner Betreuerin Dr. med. Katrin Wasser, Abteilung Neurologie, die mich sehr bei der Erfassung und Auswertung der neurologischen Daten geduldig unterstützt hat.

Weiterhin bedanke ich mich bei der gesamten Abteilung für Neuroradiologie für die freundliche Aufnahme und die praktischen Hilfestellungen im Umgang mit den verwendeten Instrumenten während vieler gemeinsamer Stunden. Martina Kellner möchte ich einen herzlichen Dank für die vorbildliche Unterstützung in allen organisatorischen Belangen aussprechen.

Meine Eltern und Freunde haben mir im Studium und bei der Ausarbeitung meiner Dissertation immer mit großer Unterstützung beiseite gestanden, dafür möchte ich mich herzlich bedanken. 